\title{
Multifaceted fibrils: self-assembly, polymorphism and functionalization $\%$
}
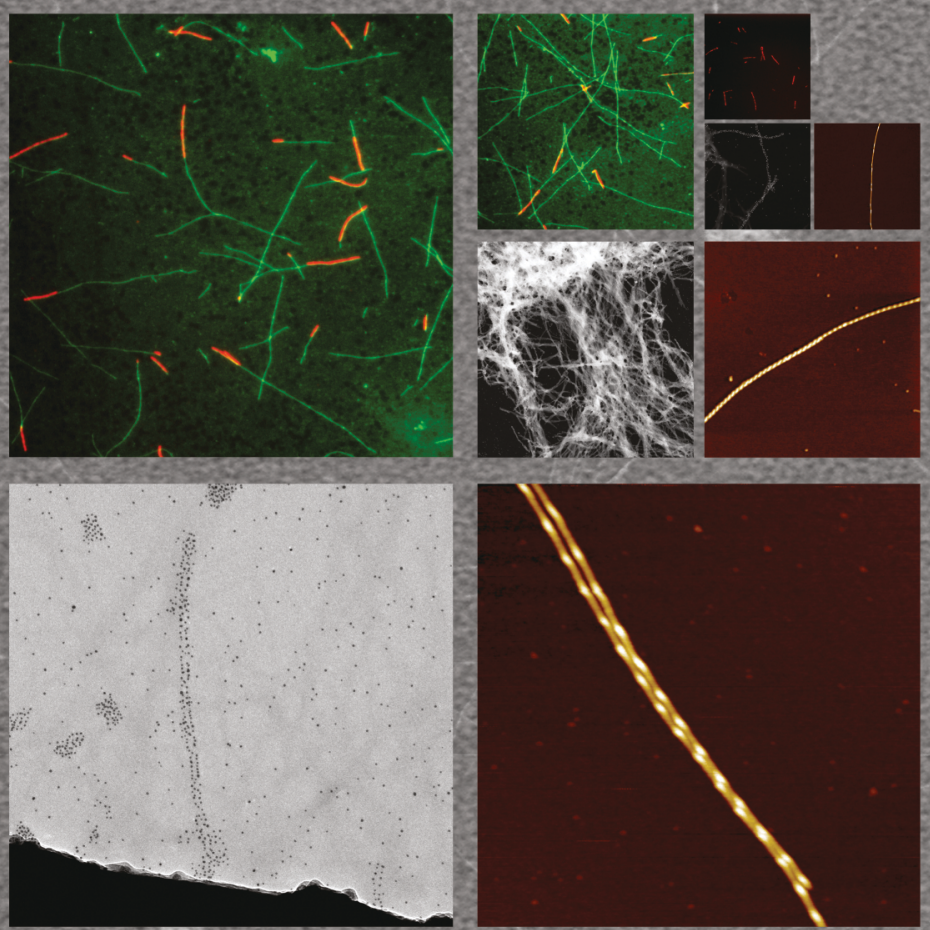

Arshdeep Sidhu

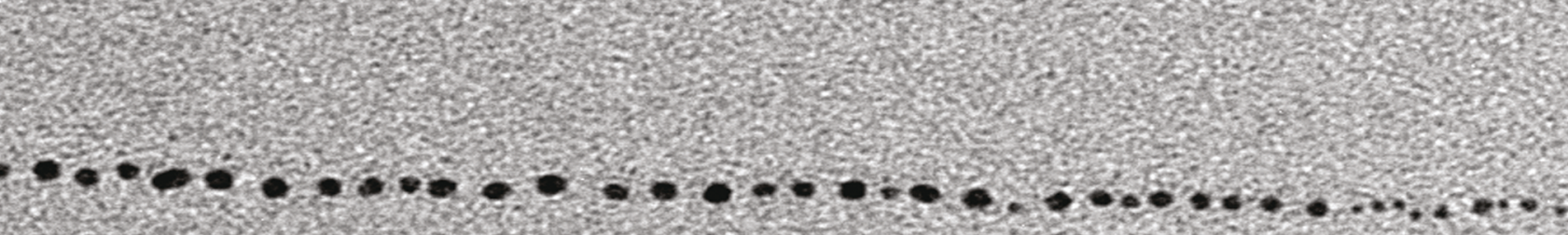

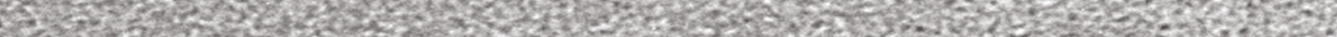
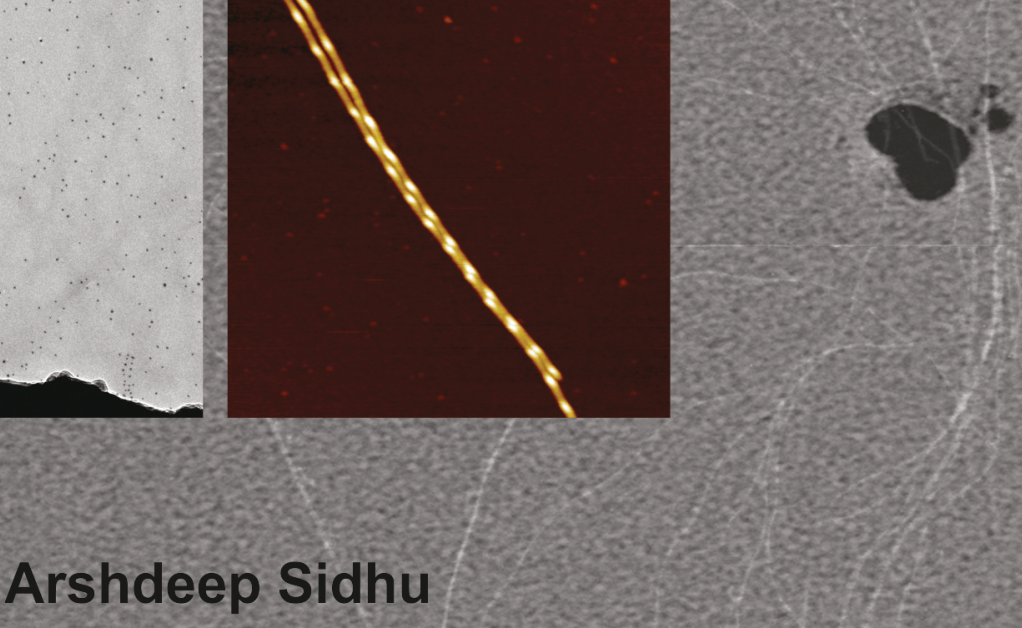

Multifaceted fibrils: self-assembly, polymorphism and functionalization

Arshdeep Sidhu 
Thesis committee members:

Prof. dr. ir. J.W.M. Hilgenkamp, University of Twente (chairman)

Prof. dr. V. Subramaniam, University of Twente (promoter)

Dr. ing. G.M.J. Segers-Nolten, University of Twente (co-promotor)

Dr. W. Hoyer, Heinrich-Heine University Düsseldorf

Dr. V. Raussens, University Libre de Bruxelles

Prof. dr. J.A. Killian, Utrecht University

Prof. dr. C. Wyman, Erasmus Universiteit Medisch centrum

Prof. dr. M.M.A.E. Claessens, University of Twente

Prof. dr. H.J.W. Zandvliet, University of Twente

The work described in this thesis was carried out at Nanobiophysics group, MESA+ Institute for Nanotechnology, Faculty of Science and Technology, University of Twente, P.O. Box 217, 7500 AE Enschede, The Netherlands. This thesis is part of NanoNextNL, a micro and nanotechnology innovation consortium of the Government of the Netherlands and 130 partners from academia and industry. More information on www.nanonextnl.nl.

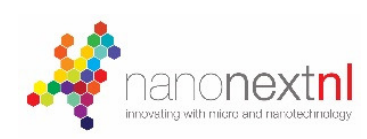

Cover design: Front, fluorescence, STEM and AFM images of aSyn fibrils. Back, graphical representation of key results of chapter 2 to chapter 6 .

ISBN 978-90-365-4024-7

DOI-10.3990/1.9789036540247

Printed by Gildeprint.

(c) Arshdeep Sidhu, 2016.

All rights reserved. 


\title{
MULTIFACETED FIBRILS: SELF-ASSEMBLY, POLYMORPHISM AND FUNCTIONALIZATION
}

\author{
DISSERTATION
}

\author{
to obtain \\ the degree of doctor at the University of Twente, \\ on the authority of the rector magnificus \\ Prof. dr. H. Brinksma \\ on account of the decision of the graduation committee, \\ to be publicly defended \\ on Wednesday the $3^{\text {rd }}$ of February 2016 at 16:45 hrs
}

by

Arshdeep Sidhu

born on $21^{\text {st }}$ of August 1984

at Palampur, India 
This dissertation has been approved by:

Prof. dr. V. Subramaniam (promotor) and

Dr. ing. G.M.J. Segers-Nolten (co-promotor)

(C) Arshdeep Sidhu, 2016

ISBN 978-90-365-4024-7 
विद्या ददाति विनयं विनयाद् याति पात्रताम्। पात्रत्वाद्धनमाप्नोति धनाद्धर्म ततः सुखम्॥ 



\section{Table of Contents}

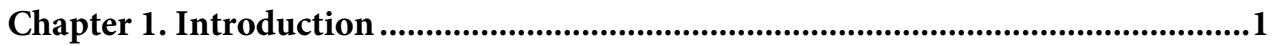

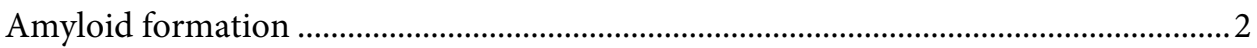

Amyloid formation kinetics.................................................................................

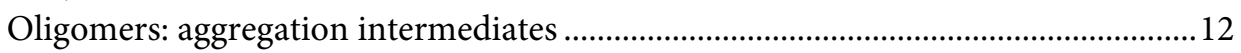

Structural polymorphism in amyloids: prion-like behavior?.......................................... 14

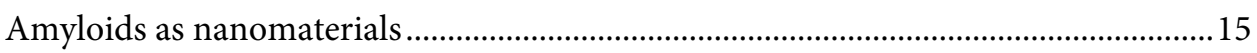

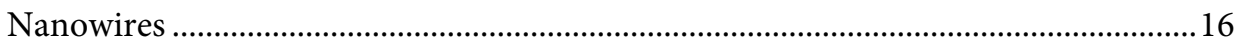

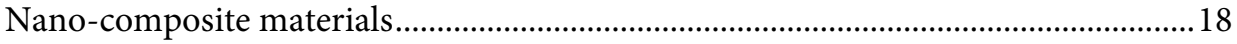

Bio-templates/matrices ......................................................................................... 19

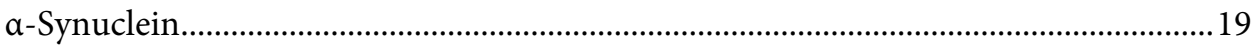

Physiological relevance of aSyn: function to association with Parkinson's disease..20

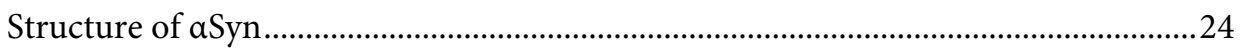

Polymorphism in aSyn and prion-like character?....................................................2

Microscopy techniques used for visualization of aSyn amyloids ...................................32

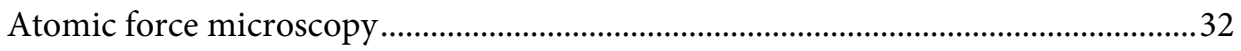

Scanning transmission electron microscopy

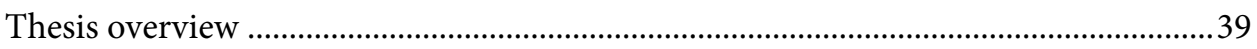

Chapter 2. Solution conditions define morphological homogeneity of $\alpha$-synuclein

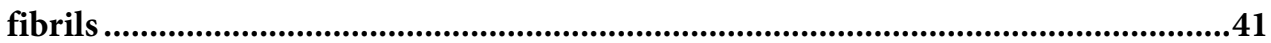

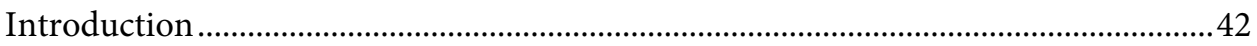

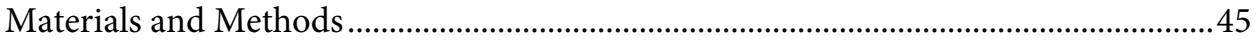

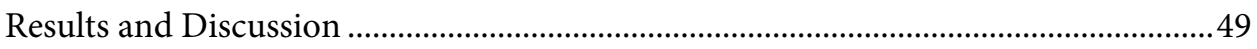

Thioflavin-T fluorescence assay ................................................................................49

AFM imaging and morphology analysis................................................................52

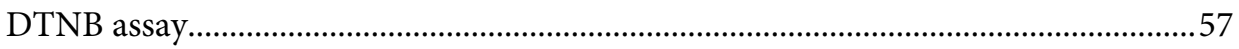

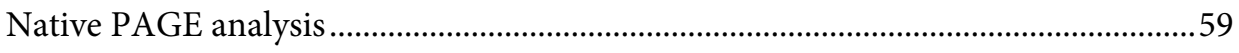

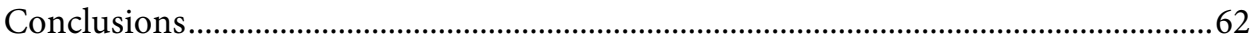

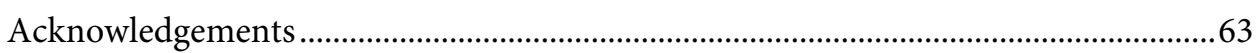


Chapter 3. Distinct mechanisms determine fibril morphology during growth and

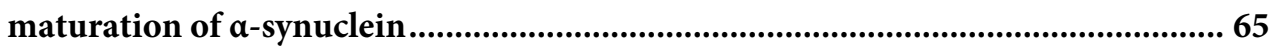

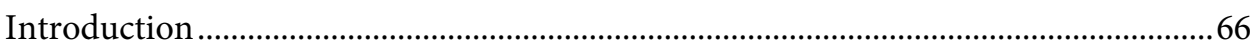

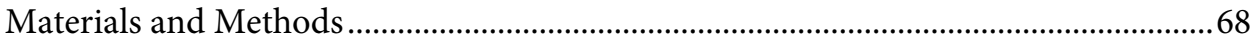

Results and Discussion .......................................................................................

Fibrillization of wt and disease mutants ....................................................................

Fibril morphology over time …………………………………………………....72

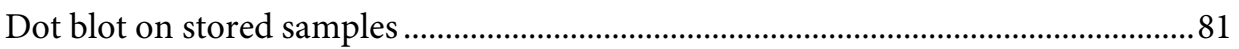

$\beta$-sheet content of plateau and matured fibrils .............................................................. 81

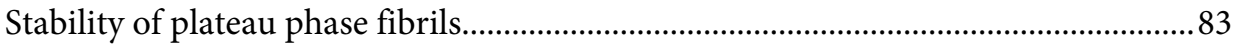

Fibrillization at low temperature …………………………................................... 84

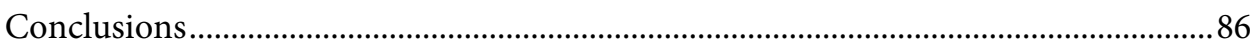

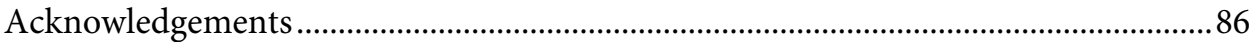

Chapter 4. Thioflavin-T fluorescence intensity depends on subtle differences in $\alpha$ synuclein fibril morphology......................................................................................... 87

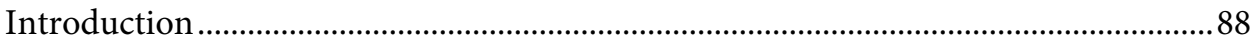

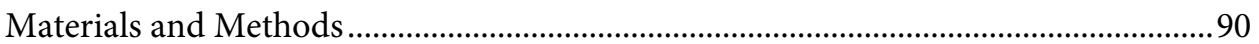

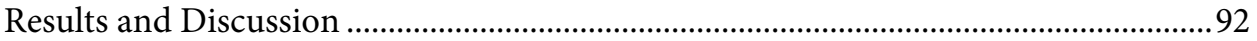

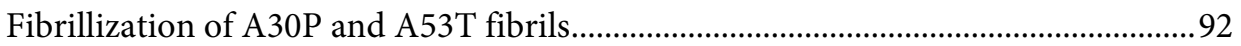

Seeded aggregations ..............................................................................................95

ThT aggregation kinetics and RMC in seeded aggregations.........................................95

ThT fluorescence lifetime and relative amplitude .......................................................97

ThT fluorescence lifetime and ThT concentration ................................................99

ThT fluorescence and fibril surface ............................................................................ 100

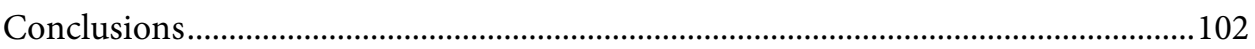

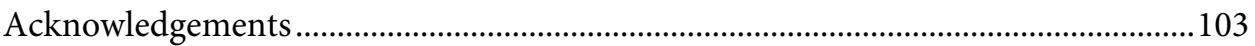

Chapter 5. Conformational compatibility is essential for heterologous aggregation

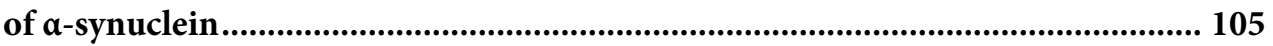

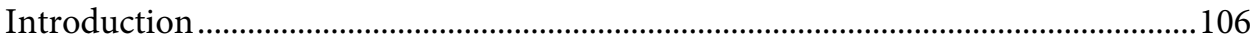

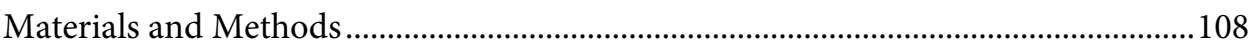

Results and Discussion .......................................................................................111

Fibrillization of wt and the disease mutants...............................................................111

Aggregation kinetics of aSyn in homologous and heterologous fibrillization .........112

Residual monomer concentration .............................................................................114

AFM imaging: fibril morphology ………………………....................................116 


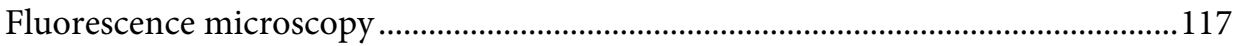

Fibril length analyses …………………………………………………………....118

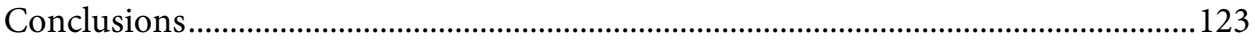

Acknowledgements ........................................................................................................123

Chapter 6. Functionalizing $\alpha$-synuclein amyloid fibrils to create self-assembled bionanostructures.......................................................................................................125

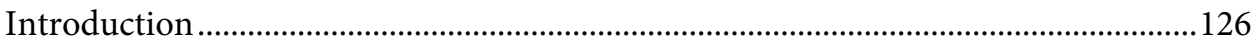

Materials and Methods ......................................................................................128

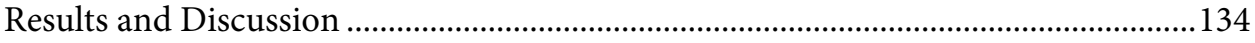

Functionalization of $\alpha$ Syn fibrils with AuNPs in solution ..........................................134

Functionalization of $\alpha$ Syn fibrils with AuNPs on a substrate .....................................141

Conductivity measurements.........................................................................................143

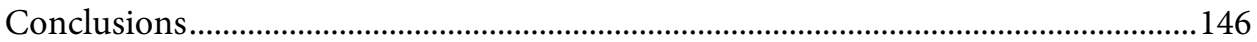

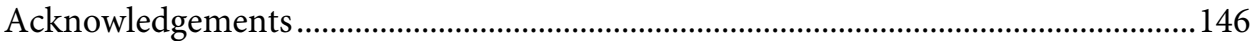

Chapter 7. Discussion and future perspectives ............................................................147

Polymorphism and amyloid studies ..........................................................................148

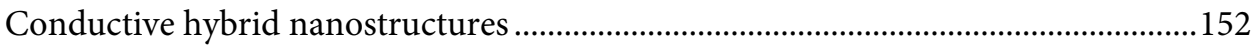

Appendix A. Risk analysis of gold nanoparticles.........................................................155

Assessment of exposure to AuNPs used in chapter 6.................................................156

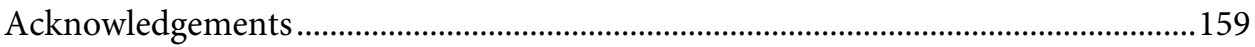

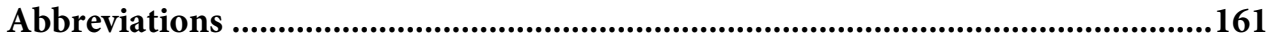

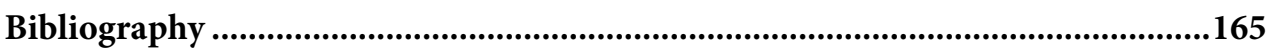

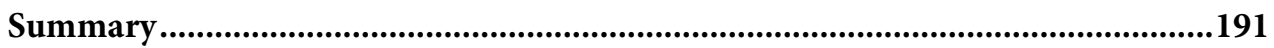

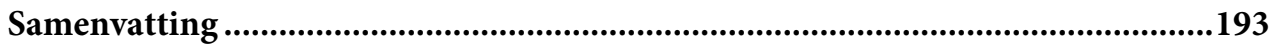

Acknowledgements.......................................................................................................197 



\section{Chapter 1}

\section{Introduction}

This chapter is a general introduction to the work presented in this thesis. Given the breadth of amyloid science, it is not possible to cover all of the topics in this brief introductory chapter. Therefore, in different sections, some of the themes relevant to the studies reported in this thesis are discussed. In the first half general features and applications of amyloid are discussed. In the second half we review the characteristics of human alpha-synuclein (aSyn) specifically, the model protein investigated in the work presented in this thesis. 


\section{AMYLOID FORMATION}

Amyloids and their classification: A large number of proteins are known to selfassemble into ordered fibrillar structures (protein polymers) named amyloids (Figure 1.1). The context of self-assembly (or aggregation or fibrillization) varies for different proteins and peptides, and can be used to classify them. Amyloids with a utility to the organism are classified as functional amyloids. Some examples of such amyloids include:

- Dragline silk formed of ADF-4 protein by Araneus diadematus: material for web spinning (Slotta, Hess et al. 2007).

- Eggshell formed of chorion protein by silk moth: provides protection (Hamodrakas, Hoenger et al. 2004).

- Hyphae ultrastructures formed of chaplins in Streptomyces coelicolor: aids in spore dispersal (Capstick, Jomaa et al. 2011).

- Pmel in vertebrate melanosomes: essential for melanosome function (Watt, van Niel et al. 2013).

- Amyloid-like hormone structures in pituitary glands: used for storage (Maji, Perrin et al. 2009).

Amyloids associated with diseases are classified as pathogenic amyloids. Examples include:

- Amyloid- $\beta(A \beta)$ : Alzheimer's disease (AD).

- $\quad \alpha$-synuclein $(\alpha \operatorname{Syn})$ : Parkinson's disease (PD).

- Amylin (IAPP): type 2 diabetes.

- Gelsolin: atherosclerosis.

- Transthyretin: peripheral amyloidosis (Eisenberg and Jucker 2012; Knowles, Vendruscolo et al. 2014).

The third emerging group in this classification is the material amyloids. These amyloids are employed in the development of new bio-nanomaterials by profiting from the spontaneous self-assembly of proteins in in vitro conditions. Some examples include 

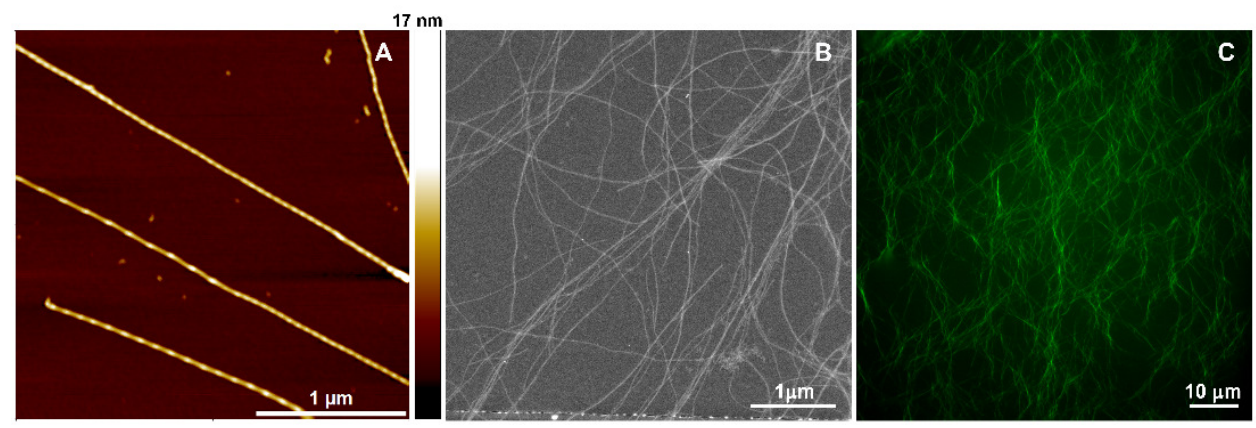

Figure 1.1 Representative images of wild type (wt) aSyn fibrils. (A) Atomic force microscopy (AFM) height image (B) Scanning transmission electron microscopy (STEM) image, magnification: 30,000 $\times(\mathrm{C})$ Widefield fluorescence image, magnification: $100 \times$.

hybrid nanowires and nano-composite materials (discussed in more detail in section: Amyloids as nanomaterials) (Gazit 2007; Mankar, Anoop et al. 2011).

General amyloid characteristics: The amyloid state of a protein is characterized by the cross $\beta$-sheet fibril diffraction pattern in X-ray studies. Two key reflections, the meridian at $0.48 \mathrm{~nm}$ and an equatorial reflection at $1 \mathrm{~nm}$, indicate the presence of 0.47 $0.48 \mathrm{~nm}$ spaced intermolecular and $1 \mathrm{~nm}$ spaced intramolecular $\beta$-strands. Irrespective of the polypeptide sequence, all the amyloids show this core structural signature. This generic backbone structure of the amyloid fibrils is believed to arise from strong backbone-to-backbone $\beta$-sheet forming interactions (hydrogen bonds) that lead to a compact and highly ordered assembly. The lateral interactions between the protein molecules in a mature fibril are governed by the side chain properties of the constituent amino acids. These interactions, hydrophobic and electrostatic, though weaker in magnitude, are specific and govern the physico-chemical properties of the fibrils (Eisenberg and Jucker 2012; Knowles, Vendruscolo et al. 2014). The presence of aromatic amino acids like tyrosine, tryptophan and phenylalanine, for example, favors self-assembly due to stabilization by $\pi$-stacking (Figure 1.2) (Gazit 2002).

Visualization of amyloids: Amyloids are routinely detected and visualized using fluorescent molecules like Thioflavin, Congo red (CR) and 8-anilinonaphthalene-1sulfonic acid (ANS) (Table 1.1 and reference (Groenning 2010)). Amyloid detection and quantitation by fluorescence spectroscopy and microscopy is based on changes in the spectral properties of these dyes on binding to $\beta$-sheet rich/hydrophobic amyloids. 
In addition to fluorescence, amyloids have also been visualized by birefringence using polarized light microscopy (Krebs, Macphee et al. 2004). Being of nanoscale dimensions amyloids can only be directly visualized, without staining or labeling, by highresolution techniques like atomic force microscopy (AFM) and electron microscopy (EM) (Note: negative staining is required for transmission electron microscopy (TEM) but not for STEM) (Figure 1.1) (Adamcik, Jung et al. 2010; Fitzpatrick, Debelouchina et al. 2013).

A

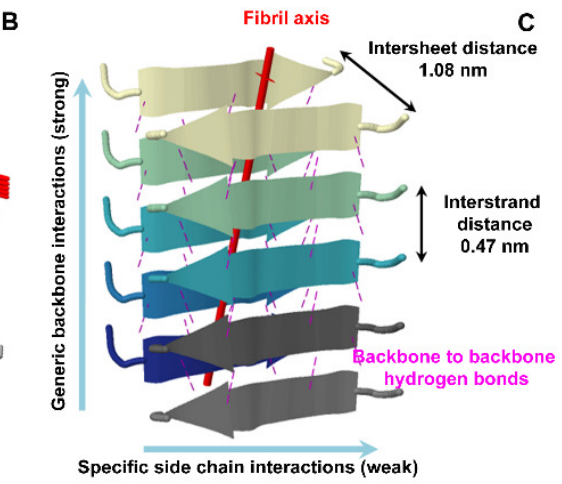

Figure 1.2 Organization of amyloids exemplified by X-ray data of aSyn fragment ${ }_{72}$ TGVTAVA $_{78}$ (Protein Data Bank-PDB ID: 4R0U). (A) Conformation of the peptide (B) Assembly of the peptide in amyloid form, top view; red dot represents fibril axis (C) Generic properties of amyloids, side view. The figures were prepared from the structural data of PDB ID: 4R0U using SPDBV v4.10 and online RCSB PDB Jmol viewer.

Importance of amyloids: The primary interest in amyloids in the recent years has been due to their association with numerous age related degenerative diseases (Eisenberg and Jucker 2012; Pulawski, Ghoshdastider et al. 2012; Knowles, Vendruscolo et al. 2014). Originally, accumulation and persistence of amyloid deposits in the affected tissue were thought to be the cause of cell death (pathogenicity) in the associated degenerative diseases. However, recent studies challenge this point of view and now the process of aggregation itself is believed to be toxic due to the formation of reactive aggregating intermediates like misfolded monomers, oligomers and protofibrils (Volles, Lee et al. 2001; Kayed, Head et al. 2003; Haass and Selkoe 2007; Fandrich 2012; Kalia, Kalia et al. 2013; Roostaee, Beaudoin et al. 2013). Therefore, knowledge of the molecular mechanisms that contribute to amyloid formation in vivo is critical to 
understand the disease etiology. This in turn is essential to develop biomarkers for early diagnosis and designing therapeutics for disease management. However, structural and biochemical studies on patient derived amyloid samples have been difficult due to insolubility of the plaques and shortage/absence of autopsy samples. To circumvent this concern, in vitro fibrillization of recombinantly produced proteins is used in many studies as the first step to understand physico-chemical properties of amyloids and the aggregation process.

Table 1.1 List of probes used for amyloid detection.

\begin{tabular}{|c|c|c|c|}
\hline Probe & Signal & $\begin{array}{l}\text { Common } \\
\text { use }\end{array}$ & Ref. \\
\hline Thioflavin-T (ThT) & Fluorescence & $\begin{array}{l}\text { In vitro } \\
\text { amyloids }\end{array}$ & $\begin{array}{c}\text { (Plotegher, Greggio et } \\
\text { al. 2014) }\end{array}$ \\
\hline Thioflavin-S (ThS) & Fluorescence & $\begin{array}{l}\text { In vivo } \\
\text { amyloids }\end{array}$ & $\begin{array}{c}\text { (Plotegher, Greggio et } \\
\text { al. 2014) }\end{array}$ \\
\hline Congo red (CR) & Birefringence & $\begin{array}{l}\text { Biopsy } \\
\text { samples }\end{array}$ & $\begin{array}{c}\text { (Elghetany, Saleem et } \\
\text { al. 1989) }\end{array}$ \\
\hline 8-Anilinonaphthalene-1-sulfonic acid (ANS) & Fluorescence & $\begin{array}{l}\text { In vitro } \\
\text { amyloids }\end{array}$ & $\begin{array}{l}\text { (Lindgren, Sorgjerd et } \\
\text { al. 2005) }\end{array}$ \\
\hline $\begin{array}{l}\text { Luminescent conjugated oligothiophenes } \\
\text { (LCOs) }\end{array}$ & Fluorescence & $\begin{array}{l}\text { Inclusion } \\
\text { bodies }\end{array}$ & $\begin{array}{c}\text { (Klingstedt, } \\
\text { Blechschmidt et al. } \\
\text { 2013) }\end{array}$ \\
\hline 9-(2,2-Dicyanovinyl) julolidine (DCVJ) & Fluorescence & Oligomers & $\begin{array}{l}\text { (Paslawski, Andreasen } \\
\text { et al. 2014) }\end{array}$ \\
\hline
\end{tabular}

Ref.: reference

In addition to the pathological perspective, amyloidogenic proteins that lack native structure are ideal to study the influence of solution conditions, small molecules and chaperones on inducing folding or misfolding in a polypeptide. Thus, amyloids have also been used as a model for protein folding and misfolding studies (Dill and Chan 1997; Dobson 2003).

Intrinsically disordered proteins and amyloids: Proteins showing partial or no tertiary and secondary structure upon in vitro purification or in silico structure prediction are named intrinsically disordered proteins (IDPs). These proteins are hypothesized to acquire structure in vivo in a context dependent manner. Various subclasses of IDPs are classified based on the extent of disorder, namely, presence of secondary structure (no tertiary structure), partly disordered (with structured regions), 
complete lack of secondary structure (Wright and Dyson 1999; Fink 2005). Intrinsic disorder provides a larger accessible surface area per number of residues than in globular form, thereby allowing multiple protein-protein interactions (Gunasekaran, Tsai et al. 2003).

A number of proteins associated with amyloid formation like $\alpha$ Syn, $A \beta$ and tau are categorized as intrinsically disordered (Breydo, $\mathrm{Wu}$ et al. 2012). Unlike globular proteins, which need to unfold and then misfold into $\beta$-sheet conformation (two steps), IDPs can form amyloids by acquiring a misfold (single step). IDPs are indicated to exhibit a broad range of Ramachandran angles, highlighting their flexible nature (Dunker, Brown et al. 2002). Consequently, IDPs' monomers can adopt multiple conformations. Under aggregation favoring conditions, many of these conformations are assumed to form distinct aggregation prone nuclei (Goldsbury, Frey et al. 2005), which has critical effect on aggregation, as discussed in the following sections.

\section{Amyloid formation kinetics}

Fibrillization is a nucleation polymerization reaction that is classically represented with a sigmoidal growth curve and described by first order kinetics (Harper and Lansbury 1997; Wood, Wypych et al. 1999; Cohen, Vendruscolo et al. 2012). However, recent studies have developed on these basic aggregation principles to provide a complex but more holistic view of molecular events in in vitro and possibly in vivo aggregation (Figure 1.3). The growth curve of amyloid formation is typically apportioned into nucleation (lag phase), elongation (exponential phase) and saturation (plateau phase) phases (Figure 1.4A). However, all the aggregation events (nucleation, fragmentation, elongation, dissociation) occur during each of the phases, albeit with different relative rates (Ferrone, Hofrichter et al. 1980; Ferrone, Hofrichter et al. 1985; Frieden 2007; Cohen, Vendruscolo et al. 2012). Some of the key features of these growth phases are as follows:

\section{Nucleation}

Nucleation refers to the formation of an aggregation prone conformation (nucleus) that can elongate to form fibrils. Although nucleation is believed to be the most important event during aggregation, it is not very well understood. The conformations sampled by an unfolded polypeptide are governed by the energy landscape of the protein 
molecule. An unfolded polypeptide is proposed to have a large degree of freedom around the Ramachandran angles (Dunker, Brown et al. 2002). Thus, numerous energetically equivalent conformations are believed to be accessible in the unfolded state (Dill and Chan 1997; Dobson 2003; Goldsbury, Frey et al. 2005). Nucleation is therefore largely agreed to be a multifactorial and stochastic process. A prerequisite for any polypeptide to adopt an aggregation prone conformation is to be in a soluble unfolded (at least partially) state, which then misfolds into an amyloidogenic conformation.

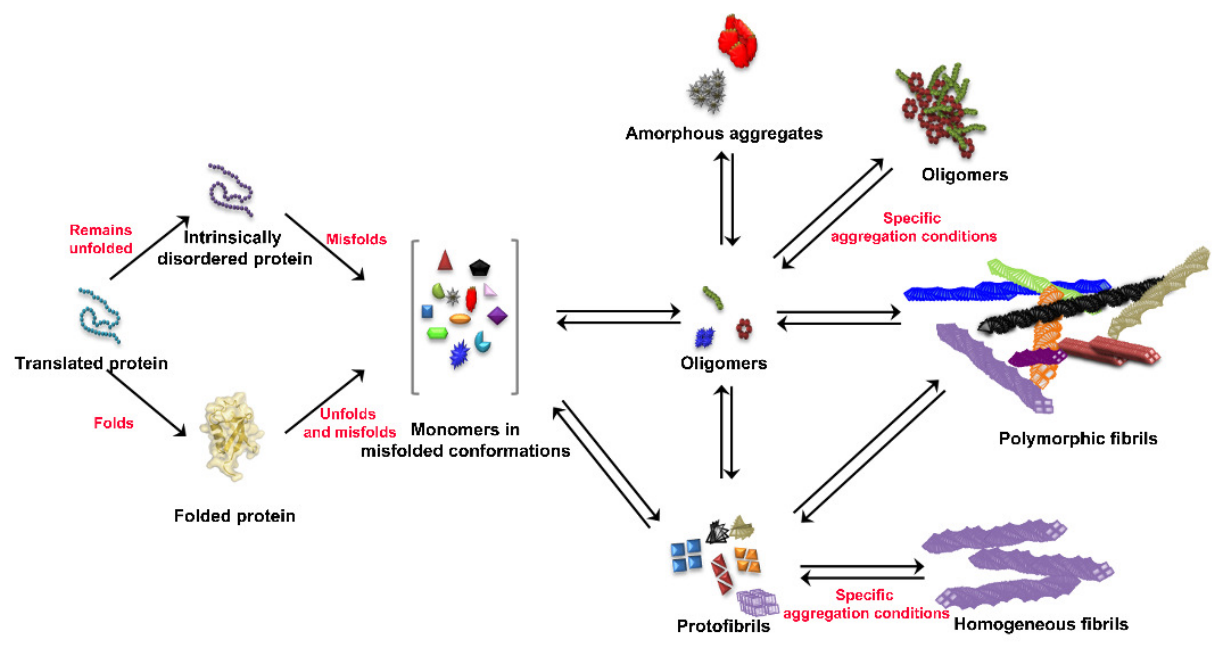

Figure 1.3 Simplistic schematic representation of protein aggregation.

Aggregation of $A \beta$ is described as a diffusion limited process with a rough energy landscape (Massi and Straub 2001). Thermodynamic fluctuations within a soluble monomer lead to the formation of an ensemble of conformations, some of which are aggregation competent. Molecular simulation studies suggest that the most populous state is not necessarily energetically the most favorable state (Pellarin, Schuetz et al. 2010). Hence, a number of conformations are most likely sampled, some of which may form multimeric species that can act as a nucleus to grow into oligomers and/or elongate into fibrils. The nuclei are believed to be held together by intermolecular hydrophobic and electrostatic interactions (hydrogen bonding and salt bridges). In in vitro aggregations with pre-formed fibrils (seeded aggregation) no lag phase is observed, highlighting that the lag phase observed in sigmoidal growth represents the nucleation 

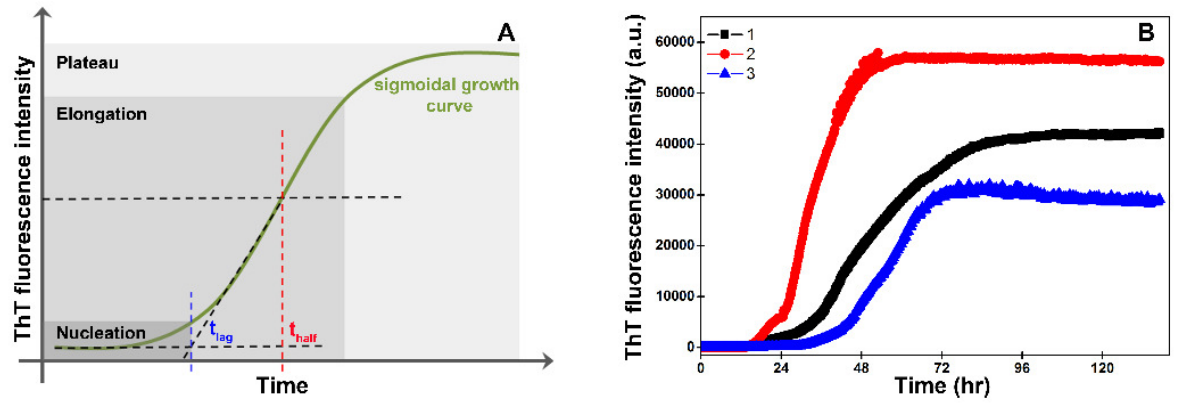

Figure 1.4 Examples of ThT growth curves. (A) A model ThT growth curve showing different phases of growth and conceptual representation of $t_{\text {lag }}$ and thalf. (B) An experimental ThT curve for A30P $\alpha$ Syn aggregation in $10 \mathrm{mM}$ Tris- $\mathrm{HCl}, 50 \mathrm{mM} \mathrm{NaCl}, 0.1 \mathrm{mM}$ EDTA at $\mathrm{pH}$ 7.4, showing clear deviation among triplicates.

process (Jarrett and Lansbury 1993). A number of extrinsic and intrinsic factors like peptide concentration, molecular crowding, solution conditions, agitation, and amino acid sequence, can influence the nucleation process.

The effect of these factors on nucleation is experimentally observed as the lag time in ThT assays, circular dichroism (CD) measurements and turbidity assays (Andreu and Timasheff 1986; Jarrett and Lansbury 1993; Bartolini, Bertucci et al. 2007). The lag time is typically described as the point of transition between the lag phase and exponential growth phase. It is an important parameter for comparison of promotion or inhibition of aggregation, and is determined by the intercept between the baseline and the extrapolated slope of the exponential growth phase $\left(\mathrm{t}_{\mathrm{lag}}\right)$. It can also be described by the time required to reach half the maximal fluorescence intensity $\left(t_{\text {half }} / t_{1 / 2} / t_{0.5}\right)$ (Powers and Powers 2006; Arosio, Knowles et al. 2015) (Figure 1.4). The influence of the above-mentioned factors on the lag time is briefly discussed as follows:

Peptide concentration: The lag time typically decreases linearly over a range of increasing peptide concentrations due to increased probability of intermolecular interactions at higher concentration. The limits of this range are expressed as the critical monomer concentration (CMC), the concentration below which no observable nucleation is detected, and supercritical concentration (SCC), above which linear concentration dependence is lost (Powers and Powers 2006). The values for CMC and SCC depend on the aggregation conditions. Under specific experimental conditions, the reported concentration range for aSyn lies between $15 \mu \mathrm{M}(\mathrm{CMC})$ and $574 \mu \mathrm{M}$ 
(SCC) (van Raaij, van Gestel et al. 2008; Giehm, Lorenzen et al. 2011). Since polypeptide concentration is a critical factor in aggregation, it is essential to correlate the neuronal concentration of the protein under study and the concentrations used in in vitro studies. Reports have suggested the neuronal concentration of aSyn in healthy cells to be 70-140 $\mu \mathrm{M}$ (van Raaij, van Gestel et al. 2008). The concentrations can however increase significantly due to gene multiplication and aberrations in protein degradation machinery (Sherman and Goldberg 2001; Shtilerman, Ding et al. 2002; Singleton, Farrer et al. 2003; Lansbury 2004). Emerging systemic studies indicate that a number of biochemical pathways affected in neurodegenerative diseases, de facto have the participant proteins in a supersaturated metastable state, which renders them prone to aggregation in the event of disturbed proteostasis (Ciryam, Kundra et al. 2015).

Molecular crowding: Molecular crowding refers to the high concentration of molecules in a given volume. It could be due to the same type of molecules, e.g. concentrated protein sample, or due to different types of proteins or additives in the given sample. Crowding due to high concentration of protein molecules is believed to facilitate intermolecular interactions and decrease the lag time in amyloid formation (Shtilerman, Ding et al. 2002). However, crowding because of the presence of a large number of different molecules, akin to the immensely crowded cellular space, presents a more complex scenario. All the molecules occupy a finite space rendering that volume inaccessible to other molecules, this results in excluded volume effects (Ralston 1990; Kuznetsova, Zaslavsky et al. 2015). Exclusion of finite solvent volume in the vicinity of a protein molecule alters the solvent protein interaction which in turns is suggested to play a major role in influencing the protein stability and conformation in the crowded milieu. The effect of crowding can be mimicked in vitro by using inert crowding agents like PEG, Ficoll, or dextrans (Shtilerman, Ding et al. 2002; Munishkina, Cooper et al. 2004). These crowding agents in addition to inducing the excluded volume effect also modify the viscosity of the solvent. Increase in solvent viscosity affects the diffusion of the protein molecules, which has been shown to have a variable effect on the lag phase (Breydo, Reddy et al. 2014).

Solution conditions: Solution conditions are reported to exert diverse effects on lag time. The choice of buffer, $\mathrm{pH}$, metal ions, small molecules and ionic strength, are all proposed to influence the aggregation kinetics in a decisive way. In general, high salt concentrations have been shown to shorten the lag time presumably due to efficient 
charge screening and thereby making hydrophobic interactions dominant (Hoyer, Antony et al. 2002; Bieschke, Russ et al. 2010; Morel, Varela et al. 2010; Pronchik, He et al. 2010; Wang, Boddapati et al. 2010; Lu, Ardah et al. 2011; Breydo, Wu et al. 2012; Viles 2012; Buell, Hung et al. 2013; Buell, Galvagnion et al. 2014; Semerdzhiev, Dekker et al. 2014; Sidhu, Segers-Nolten et al. 2014). Another important factor suggested to affect protein conformation is the presence of metal ions (Nath, Sammalkorpi et al. 2012). $\alpha$ Syn is shown to have affinity to a number of metal ions like iron, copper, zinc and aluminium. However, only copper is reported to have three specific binding sites in the protein sequence. The $\mathrm{N}$-terminal motif, ${ }_{1} \mathrm{MDVFM}_{5}$ has a high affinity for $\mathrm{Cu}(\mathrm{I})$ binding via sulfur atoms to Met- 1 and Met-5 (Miotto, Rodriguez et al. 2014). Two other sites with affinity to $\mathrm{Cu}(\mathrm{I})$ include His-50 and the C-terminal methionines in $\mathrm{M}(\mathrm{X})_{\mathrm{n}} \mathrm{M}$ motif (Met-116 and Met-127) (Sung, Rospigliosi et al. 2006; Camponeschi, Valensin et al. 2013).

Agitation: Agitation is shown to reduce the lag time in $\alpha$ Syn and insulin aggregation (Nielsen, Khurana et al. 2001; Fink 2006; Knowles, Waudby et al. 2009). Studies on A $\beta$ have shown that in addition to increasing the probability of molecular interaction and thus primary nucleation, agitation contributes significantly towards the secondary process of fragmentation (Cohen, Linse et al. 2013).

Mutations: Single point mutations can substantially affect the aggregation kinetics (Polymeropoulos, Lavedan et al. 1997; Fink 2006). Most of the disease associated point mutations are known to promote aggregation and have a decreased lag time (Conway, Harper et al. 1998; Greenbaum, Graves et al. 2005; Ghosh, Mondal et al. 2013; Ghosh, Sahay et al. 2014). Substitution of a single amino acid can alter the hydrophobicity, the propensity to acquire $\beta$-sheet structure along with the overall charge of the polypeptide. An analysis of the combined effect of these factors on aggregation rates of different mutant peptides showed comparable results. These results highlight that mutations modify broad physico-chemical interactions in a molecule rather than a point interaction (Chiti, Stefani et al. 2003). In addition, the extent of perturbation of native interactions is suggested to be highly dependent on the position of the mutation in the sequence (Chiti, Taddei et al. 2002). 


\section{Elongation}

Once an aggregation prone critical nucleus is formed, it is believed to grow at the ends to form the fibrillar structures, observable in AFM and EM (Khurana, Ionescu-Zanetti et al. 2003; Lashuel and Wall 2005; Adamcik, Jung et al. 2010). The transition from nucleation to elongation is characterized by elongation of stable nuclei with faster addition, than dissociation, of monomers at the end(s). Two mechanisms are proposed for the elongation of the nuclei; (1) a single step mechanism, wherein a preformed structural unit of the fibril adds to the end. In this scenario, the soluble protein could adopt $\beta$-sheet conformations that can directly add to the growing end or the soluble protein could assemble into oligomeric units, which elongate the fibril. (2) A two-step or dock and lock mechanism, in which the soluble protein molecules are tethered to the growth competent end (dock) and fold themselves on the existing end (lock). In molecular simulation studies, the docking step is shown to be fast and mediated by hydrophobic interactions while the locking step is suggested to be slower as it involves reconfiguration of the polypeptide fold in sync with the template molecule (Esler, Stimson et al. 2000; Straub and Thirumalai 2011; Knowles, Vendruscolo et al. 2014). A comparison between the theoretical and experimentally observed rate of elongation in $\mathrm{A} \beta_{1-40}$ revealed that only a fraction $\left(\approx 10^{-4}\right)$ of collisions occurring with the fibril result in addition and growth of the fibril. This disparity is understood to be due to the transient interaction of the disordered monomer with the fibril end in the dock stage. Subsequently, only if sufficient molecular contacts are made the molecule can lock to the end, else it diffuses away (Qiang, Kelley et al. 2013).

The rate of elongation may not be uniform due to formation of an unusual yet stable conformation at the growing end. Such a conformation might convert to a proper conformation, leading to intermittent growth or stall the growth altogether by capping the end (Kellermayer, Karsai et al. 2008; Ferkinghoff-Borg, Fonslet et al. 2010; Qiang, Kelley et al. 2013, Wordehoff, Bannach et al. 2015). The ends of the fibrils are thus crucial units for elongation and the rate of growth should be proportional to the number of fibril ends in a reaction. Although this simplistic view captures the essence of the importance of fibril ends, it is often not adequate to explain the experimental observations. The exponential growth of the fibrils in the elongation phase suggests influence of additional mechanism(s) that increase the number of elongating aggregates. 
Like nucleation, at the molecular level elongation is a complex process with multiple microscopic events that influence the overall elongation rate (Cohen, Vendruscolo et al. 2012; Arosio, Knowles et al. 2015). A simple means to produce new ends is fragmentation of the existing fibrils. Fragmentation is facilitated by agitation of the sample and has been shown to result in faster rate of elongation (Knowles, Waudby et al. 2009; Xue, Hellewell et al. 2009; Shvadchak, Claessens et al. 2015).

In addition to growing on the ends, the surface of the fibrils is also proposed to act as a nucleation surface. This is known as secondary nucleation and has been important in understanding experimentally observed aggregation kinetics in $\mathrm{A} \beta$ and $\alpha \mathrm{Syn}$ (Fodera, Librizzi et al. 2008; Cohen, Linse et al. 2013; Buell, Galvagnion et al. 2014). Therefore, detailed analysis of the growth curves is essential to infer the predominant growth mechanism in the given solution conditions. Such analyses facilitate a better understanding of the influence of different parameters on amyloid growth (Knowles, Waudby et al. 2009; Cohen, Vendruscolo et al. 2012; Buell, Galvagnion et al. 2014).

\section{Plateau phase}

The leveling off of the growth curve indicates diminished rate of conversion of soluble peptides into amyloid form and is called as the end or plateau phase. At this phase, the rate of addition of monomers to the active ends of fibrils is assumed equal to the dissociation rate of the monomers. This is largely due to depletion of soluble monomers that can add to the fibril ends (Cohen, Vendruscolo et al. 2012). Classically, this is supposed to be an equilibrium state and the amount of monomers left in the reaction are thought to reflect the minimum amount of soluble protein required to form amyloid aggregates (CMC). It is experimentally determined as residual monomer concentration (RMC). However, recent studies show that the plateau phase is a quasi-equilibrium state and that the fibrils continue to grow albeit at a much slower rate. Further, studies also show that fibrils themselves undergo slow transitions over time in vitro as well as in vivo (Vilar, Chou et al. 2008; Guilliams, El-Turk et al. 2013; Ma, Komatsu et al. 2013; Nystrom, Psonka-Antonczyk et al. 2013).

\section{Oligomers: aggregation intermediates}

Oligomers are intermediate species between the monomeric state of the protein and their fibrillar form. Only a fraction of protein molecules are believed to exist in the 
oligomeric state during the entire aggregation process, thus making their isolation and characterization extremely difficult (Arosio, Knowles et al. 2015). Experimentally numerous structural conformations are observed for oligomers. Like fibril formation, this could be due to multiple assembly pathways (Uversky 2010; Fandrich 2012; Paslawski, Mysling et al. 2014; Chen, Drakulic et al. 2015). The most common distinction proposed between the oligomer populations is the on-pathway and offpathway oligomers. The on-pathway oligomers are expected to be actively involved in fibrillization and are thus incorporated into the fibrils. The off-pathway oligomers on the other hand, are presumed to be kinetically trapped assemblies that do not transform into fibrils (Paslawski, Andreasen et al. 2014; Paslawski, Mysling et al. 2014; Andreasen, Lorenzen et al. 2015). Some evidence suggests that oligomers could be more neurotoxic than the monomers or fibrils. Thus to better understand these sparsely populated species, protocols have been optimized to convert a higher fraction of monomers into oligomers (Herrera, Chesi et al. 2008; van Rooijen, Claessens et al. 2009; Giehm, Svergun et al. 2011; Chen, Drakulic et al. 2015). Structural studies show that oligomers from a number of amyloidogenic proteins like $\alpha$ Syn, $A \beta$, lysozyme, $\beta_{2}$-microglobulin have anti-parallel arrangement of $\beta$-sheets as opposed to parallel arrangement found in their fibrillar form (Fabian, Gast et al. 2008; Cerf, Sarroukh et al. 2009; Celej, Sarroukh et al. 2012; Zou, Li et al. 2013; Chen, Drakulic et al. 2015). However, oligomers can also have random coil and partial $\alpha$-helical conformations (Apetri, Maiti et al. 2006; Sandberg, Luheshi et al. 2010; Trexler and Rhoades 2012). Oligomers do not significantly enhance the ThT fluorescence, reflecting the negligible ThT fluorescence intensity observed during the lag phase when oligomers are thought to be most populous (Apetri, Maiti et al. 2006; Chen, Drakulic et al. 2015). The standard biochemical method to detect oligomers is based on the A11 antibody reaction. This antibody reacts with oligomers of a number of amyloidogenic proteins, highlighting a common structural motif in the known oligomers (Kayed, Head et al. 2003). Several environmental factors can influence oligomerization, however, curiously the extent of conversion of soluble polypeptide into oligomers is shown to be independent of the protein and salt concentration (Hong, Han et al. 2011; Breydo, Wu et al. 2012; Paslawski, Andreasen et al. 2014). Toxicity of the oligomers is believed to be due to their destructive interaction with the cell membranes. Numerous studies have investigated oligomer-membrane interactions; however, the exact mechanism of damage is still 
elusive (Davidson, Jonas et al. 1998; Haass and Selkoe 2007; Giehm, Svergun et al. 2011; Breydo, Wu et al. 2012; Snead and Eliezer 2014; Andreasen, Lorenzen et al. 2015). Broadly, toxicity is proposed to be due to pore formation in the membrane (amyloid pore hypothesis) or extraction of the lipid molecules (membrane damage) (Volles and Lansbury 2002).

\section{Structural polymorphism in amyloids: prion-like behavior?}

Structural polymorphism refers to the capacity of a polypeptide chain to form fibrils of different morphologies. The distinct morphologies could arise due to different folds (conformations) of the protein monomers or differences in the intermolecular contacts of the nucleating monomers. As discussed earlier, the conformations adopted by the soluble monomer depend on a number of factors including solution conditions, molecular crowding, mutations etc. Morphological analyses of the plateau phase fibrils, based on ThT assays, by AFM and EM routinely shows polymorphic fibrils (Jimenez, Nettleton et al. 2002; Khurana, Ionescu-Zanetti et al. 2003; Heise, Hoyer et al. 2005; Fandrich, Meinhardt et al. 2009). Polymorphism is believed to be inherent and reflects the stochastic nature of amyloid formation. It makes interpretation of experimental results from structural and toxicity studies challenging. Therefore, aggregation protocols have been optimized to form near homogeneous fibril samples (Heise, Hoyer et al. 2005; Bousset, Pieri et al. 2013; Sidhu, Segers-Nolten et al. 2014). Structural studies on such samples indicate different backbone conformations, electrostatic interactions and salt bridges in distinct polymorphs (Heise, Hoyer et al. 2005; Petkova, Leapman et al. 2005; Paravastu, Leapman et al. 2008; Gath, Bousset et al. 2014). Moreover, in vivo studies show differences in toxicity profiles between specific polymorphs (Petkova, Leapman et al. 2005; Bousset, Pieri et al. 2013).

Polymorph based differences in toxicity coupled with cell-to-cell transmission of aggregates in cell cultures and animal models has invoked the prion-like hypothesis for amyloids (George, Rey et al. 2013; Goedert, Spillantini et al. 2013). Prions are misfolded protein molecules that aggregate and propagate in a template-assisted manner by recruiting normal cellular counterparts. Prion proteins can adopt diverse conformations that are believed to give rise to different strains. These strains are reported to show characteristic growth and infectivity (Tanaka, Collins et al. 2006; Shikiya, Ayers et al. 2010; Colby and Prusiner 2011). The observed polymorphism in 
amyloids is compared to the strains of prions, both of which show nucleation and competitive growth (Wood, Wypych et al. 1999; Tanaka, Collins et al. 2006; Shikiya, Ayers et al. 2010). Furthermore like prions, amyloids are suggested to template aggregation and spread to neighboring cells (Petkova, Leapman et al. 2005; Bousset, Pieri et al. 2013; George, Rey et al. 2013; Goedert, Spillantini et al. 2013; Jucker and Walker 2013). Thus, in essence, amyloids seem to share many of the cardinal features of prions (Dunning, George et al. 2013; Goedert, Falcon et al. 2014).

\section{AMYLOIDS AS NANOMATERIALS}

Material properties of amyloids have been extensively investigated in an effort to understand the basis for the persistence of amyloid fibrils over extended periods in pathogenic conditions. The amyloid form of the protein is reported to be highly stable; demonstrated by resistance to proteolysis, changes in $\mathrm{pH}$, salt concentration, tolerance to high pressure (up to $500 \mathrm{MPa}$ ) and temperature (up to $100^{\circ} \mathrm{C}$ ) (Chiti and Dobson 2006; Meersman and Dobson 2006). Amyloids are also proposed to have exceptional mechanical properties, for example, insulin fibrils were suggested to have tensile strength akin to steel (0.6 GPa) and mechanical stiffness similar to silk (3.3 GPa) (Smith, Knowles et al. 2006). These remarkable material properties coupled with spontaneous self-assembly in micro-molar protein concentrations in vivo may provide a poor prognosis for amyloid diseases, but offer a unique avenue for development of novel bionanomaterials.

In addition to the above mentioned material properties, some of the key features which make amyloids attractive as nanomaterials include:

- The inherent nanoscale dimensions: amyloid fibrils are typically $10 \mathrm{~nm}$ wide in diameter and can be several micrometers long.

- Spontaneous self-assembly into fibrils and higher order structures like films, gels, networks etc.

- Ease of functionalization by biochemical modifications of the constituent amino acids.

A new discipline of amyloid research, material amyloid, has consequently emerged that explores the application of amyloids as nanomaterial. Besides natural amyloids, 
synthesized di- or oligo-peptides with a propensity to self-assemble into nanostructures resembling tubes, spheres etc. have also been shown to be useful nanomaterials (Gazit 2007). Key examples of amyloid based nanomaterials are as follows:

- Nanowires

- Nano-composite materials

- Bio-templates/matrices

\section{Nanowires}

Conductive nanowires: Amyloids being proteinaceous are poor conductors; however, numerous studies have reported functionalization of amyloid fibrils with conductive elements to fabricate nanowires (Table 1.2). The produced nanowires have been used as sensors to detect nucleic acids, proteins, viruses and biologically relevant small molecules (Rosi and Mirkin 2005; Gazit 2007). The nanoscale dimensions of amyloid fibrils, about $10 \mathrm{~nm}$ in diameter, positions them as excellent self-assembled scaffold material. The produced nanowires are co-axial, with a fibrillar protein core wrapped in a conductive shell. The conductive moieties can be attached via modification of one of the protein groups like primary amines $\left(-\mathrm{NH}_{2}\right.$ at the $\mathrm{N}$-terminus and the side chains of lysines and arginines), carboxyl groups (- $\mathrm{COOH}$ at the $\mathrm{C}$-terminus and the side chains of aspartic and glutamic acid residues) and sulfhydryl groups (-SH on the side chain of cysteines). Depending on the application of the nanowire, different conductive moieties can be chosen for functionalization of amyloid fibrils. Two of the commonly employed conductive elements are briefly discussed:

Metal nanoparticles: Metal nanoparticles are the most common conductive elements for conjugation to the amyloid fibrils. Routinely used metal particles include nanoparticles of gold, silver, palladium, copper, cobalt etc. (Banerjee, Yu et al. 2003; Scheibel, Parthasarathy et al. 2003; Colby, Hulleman et al. 2008; Lee, Choe et al. 2011). The nanoparticles can be of varying size, which is selected based on the intended application of the hybrid bio-nanostructure. Among the metal nanoparticles, gold nanoparticles (AuNPs) are most widely used for metallization due to the relative ease of surface functionalization by a range of molecules like thiolates, dithiolates, amines, carboxylates, cyanides etc. (Love, Estroff et al. 2005). 
Conjugated polyelectrolytes: Conjugated polyelectrolytes (CPEs) are organic polymers with a dissociable electrolytic group (e.g. sulfonate $\mathrm{SO}_{3}^{-}$, carboxylate $\mathrm{COO}$, and phosphonate $\mathrm{PO}_{3}{ }^{2-}$ ) as a side chain. This results in the formation of a charged and thus electrically conductive species when dissolved in aqueous solvents. Their hydrophobic backbone and hydrophilic side chain imparts an amphiphilic character and a tendency to aggregate in solution. The photo-physical properties of CPEs depend on the solvent conditions. Usually, CPEs have absorption maxima in the range of 400$550 \mathrm{~nm}$ and fluorescence emission maxima in the range of 440-600 nm. Owing to their opto-electric redox properties, CPEs like PEDOT/PSS, PPF, APFO-12, PONT1 and 2 have been co-aggregated with amyloids of insulin and recombinant spider silk to form hybrid fibrils exhibiting conductive and luminescent properties (Hamedi, Herland et al. 2008; Herland, Thomsson et al. 2008; Tanaka, Herland et al. 2008; Bjork, Herland et al. 2010; Muller, Jansson et al. 2011).

Table 1.2 List of amyloid based nanowires.

\begin{tabular}{|c|c|c|c|c|}
\hline Protein & $\begin{array}{c}\text { Conductive } \\
\text { moiety }\end{array}$ & $\begin{array}{c}\text { Nanowire } \\
\text { specification }\end{array}$ & Functionality & Ref. \\
\hline$\alpha S y n$ & PcTS & $\mathrm{L}=400 \mathrm{~nm}-1.4 \mu \mathrm{m}$ & $\begin{array}{c}\text { Conductive and light } \\
\text { sensitive }\end{array}$ & $\begin{array}{l}\text { (Choi, Kim et al. } \\
\text { 2011) }\end{array}$ \\
\hline $\begin{array}{l}\text { Bovine } \\
\text { insulin }\end{array}$ & PEDOT/PSS & $\begin{aligned} \mathrm{L}= & \approx 100 \mu \mathrm{m}, \mathrm{D}= \\
& \approx 10 \mu \mathrm{m}\end{aligned}$ & $\begin{array}{l}\text { Electro-optical } \\
\text { properties }\end{array}$ & $\begin{array}{c}\text { (Herland, Bjork et } \\
\text { al. 2005) }\end{array}$ \\
\hline$\alpha \operatorname{Syn} E 46 K$ & $\mathrm{Pd}$ & $\begin{array}{c}\mathrm{L}=\text { several } \mu \mathrm{m}, \mathrm{D}= \\
90-110 \mathrm{~nm}\end{array}$ & Conductive & $\begin{array}{l}\text { (Colby, Hulleman } \\
\text { et al. 2008) }\end{array}$ \\
\hline$\alpha \operatorname{Syn} E 46 K$ & $\mathrm{Au}$ & $\mathrm{D}=100 \mathrm{~nm}$ & Not mentioned & $\begin{array}{l}\text { (Colby, Hulleman } \\
\text { et al. 2008) }\end{array}$ \\
\hline$\alpha \operatorname{Syn} E 46 K$ & $\mathrm{Cu}$ & $\mathrm{D}=90 \mathrm{~nm}$ & Not mentioned & $\begin{array}{l}\text { (Colby, Hulleman } \\
\text { et al. 2008) }\end{array}$ \\
\hline Sup35 & $\mathrm{Au}$ & $\begin{array}{c}\mathrm{L}=\text { several } \mu \mathrm{m}, \mathrm{D}= \\
80-200 \mathrm{~nm}\end{array}$ & Conductive & $\begin{array}{c}\text { (Scheibel, } \\
\text { Parthasarathy et al. } \\
\text { 2003) }\end{array}$ \\
\hline$\alpha \operatorname{Syn} A 53 C$ & $\mathrm{Au}$ & $\begin{array}{c}\mathrm{L}>10 \mu \mathrm{m}, \mathrm{D}=57.3 \\
\mathrm{~nm}\end{array}$ & Conductive & $\begin{array}{l}\text { (Lee, Choe et al. } \\
\text { 2011) }\end{array}$ \\
\hline Actin & $\mathrm{Au}$ & $\begin{array}{c}\mathrm{L}=1-4 \mu \mathrm{m}, \mathrm{H}=80- \\
200 \mathrm{~nm}\end{array}$ & Electrical conductivity & $\begin{array}{c}\text { (Patolsky, } \\
\text { Weizmann et al. } \\
\text { 2004) }\end{array}$ \\
\hline
\end{tabular}

Photonic nanowires: In addition to electronically conductive nanowires, photonic nanowires that can transmit photons over a finite distance can also be prepared. The common choice of transmitting elements for photonic wires includes fluorophores, 
quantum dots and derivatives of naturally photo/electronic porphyrin molecules. Nanowires prepared from amyloids of hemoglobin A have been indicated to exhibit conductive, photodynamic and photovoltaic properties (Lee, Kang et al. 2010). Also, conjugation of phthalocyanine tetrasulphonate (PcTS) with oligomers of aSyn prepared in the presence of ferric ions are suggested to form photoelectric hybrid material with a conductance of up to $270 \mathrm{AV}^{-1}$ (Choi, Kim et al. 2011). The fabrication of photonic nanowires is however not easy. Some of the challenges in their development include obtaining a high quantum yield, photo-stability and the apt spacing of fluorophores in the right sequence (i.e. with respect to their excitation energy to ensure unidirectionality of energy transfer) (Heilemann, Tinnefeld et al. 2004).

\section{Nano-composite materials}

Functional amyloids like curli, chaplins, hydrophobins and chorion are organized into protective films that provide structural support to the parent organisms (enterobacteria, streptomycetes, fungi, insects, respectively) (Cherny and Gazit 2008). Inspired by these natural bio-films, similar film-like assemblies from amyloid fibrils doped with a range of inorganic molecules have been fabricated in vitro. Some examples of such functionalized nano-composite bio-films are as follows:

- Nano-fibrils of lysozyme and $\beta$-lactoglobulin ( $\beta$-lac), functionalized with ThT, developed into rigid and ordered macroscopic films (Knowles, Oppenheim et al. 2010).

- Fibrils of $\beta$-lac used as a reducing surface to prepare $100 \mathrm{~nm}$ thick gold micro flakes with potential application for flexible conductors (Zhou, Saha et al. 2015).

- Bone like composites in organization and structural-functional properties, using hydroxyapatite/brushite and amyloids (Li, Born et al. 2014).

- Biodegradable composites of graphene and $\beta$-lac that show conductive behavior as well as sensitivity to humidity and enzymatic activity, for use as sensors (Li, Adamcik et al. 2012).

In addition to the distinct advantage of bottom up fabrication of these nano- to macroscopic composites, they demonstrate the physico-chemical properties of the key constituent materials (Li, Bolisetty et al. 2013). 


\section{Bio-templates/matrices}

Being proteinaceous in nature, amyloids are generally expected to show better compatibility with biological systems in comparison to inorganic materials. Moreover, the possibility of precise functionalization (to mimic physiological conditions) and their ability to form networks (hydrogels) makes them promising substrates for matrices for 3D cell culture (Kasai, Ohga et al. 2004; Gras, Tickler et al. 2008). In agreement, amyloid based substrates have been suggested to show enhanced cell adhesion, proliferation and improved differentiation in several studies (Kasai, Ohga et al. 2004; Horii, Wang et al. 2007; Bongiovanni, Scanlon et al. 2011). Although, there is concern regarding the safety of amyloids due to their association with degenerative diseases, extensive cell growth and differentiation oriented experiments, particularly with amyloids not associated with disease are under investigation to explore their potential for use as templates.

Theoretically, any polypeptide sequence has been proposed to be able to selfassemble into amyloid fibrils (Dobson 2003). Since the formation of amyloids is suggested to be an inherent property, it imparts some generic features to the amyloids. Some of these common features and applications have been discussed in the preceding sections. However, the work presented in this thesis focusses on aSyn amyloid fibrils. In the following sections, we review the general features, relevance, structure and the prion-like hypothesis for aSyn fibrils.

\section{$\alpha$-SYNUCLEIN}

a-Synuclein (aSyn) is a 140 amino acid eukaryotic protein, mainly expressed in neuronal tissue (Maroteaux, Campanelli et al. 1988). It is primarily associated with the pathogenesis of Parkinson's disease (PD). It is transcribed from the SNCA gene, at chromosome 4q21 and is also known as PD1, non A $\beta$ component of amyloid precursor (NACP), PARK1 and PARK4 (Gene ID: 6622). $\alpha$ Syn is a member of the synuclein family of proteins. There are three synuclein proteins in the family: $\alpha, \beta$ and $\gamma$ (Table 1.3). The synuclein family proteins are characterized by highly conserved 11 residue repeat sequences in the amino-terminus that are proposed to adopt an $\alpha$-helical fold on binding to membranes. The $\mathrm{C}$-terminus of all three synucleins is rich in acidic residues (George 2002). In addition to the homologous sequence variants in the form of $\beta$ and $\gamma$ synucleins, aSyn has four reported isoforms. These isoforms (SNCA 126, SNCA 112, 
SNCA 98 and aSynL) arise form alternative splicing of the five protein coding exons and one 3'UTR exon (Ueda, Saitoh et al. 1994; Campion, Martin et al. 1995; Beyer, Domingo-Sabat et al. 2008, Rhinn, Qiang, et al. 2012).

Table 1.3 Summary of sequence alignment of human synuclein family proteins. Alignment was done using online UniProt align tool based on Clustal Omega program (ClustalO).

\begin{tabular}{cccccc}
\hline Protein & UniProt & Gene name & \multicolumn{3}{c}{ With respect to $\alpha$ Syn } \\
\cline { 3 - 5 } & ID & & Identity & Identical residues & Similar residues \\
$\alpha S y n$ & P37840 & SNCA, NACP, PARK1 & $100 \%$ & 140 & 140 \\
$\beta S y n$ & Q16143 & SNCB & $59 \%$ & 86 & 26 \\
$\gamma$ Syn & O76070 & SNCG, BCSG1, PERSYN, & $50 \%$ & 70 & 30 \\
& & PRSN & & & \\
\hline
\end{tabular}

\section{Physiological relevance of $\alpha$ Syn: function to association with Parkinson's disease}

Since the discovery of the fibrillar form of aSyn as the major component of the Lewy bodies (LBs) and Lewy neurites (LN), aSyn function and in vivo characteristics have been a major focus for understanding the pathogenesis of PD. Clinically, PD is characterized by movement disorders: bradykinesia, rigidity, tremors, postural instability and non-motor symptoms: sleep problems, depression, cognitive impairment and fatigue ${ }^{1}$. The presence of LBs and LN in post mortem biopsies accompanying loss of dopaminergic neurons in the substantia nigra, is the hallmark for diagnosis of PD and other neurodegenerative diseases called synucleinopathies (Spillantini, Crowther et al. 1998; Takeda, Mallory et al. 1998). The exact function of aSyn in vivo is not known. Therefore, the association of aSyn with PD is due to convergent evidence like:

- Elevated levels of aSyn in PD patients.

- Multiplication of the SNCA gene (that increases the protein level).

- Autosomal dominant inheritance of point mutations in aSyn related to early onset of PD.

- Recapitulation of a number of disease symptoms in model systems upon overexpression of aSyn.

\footnotetext{
${ }^{1}$ http://www.ninds.nih.gov/disorders/parkinsons_disease/parkinsons_disease.htm
} 
Clinical studies distinguish two types of PD, the sporadic form with undefined etiology and the familial form associated with genetic mutations. The familial form of $\mathrm{PD}$, characterized by an early age of onset, is suggested to arise by autosomal dominant point mutations or duplication of the SNCA locus (Polymeropoulos, Lavedan et al. 1997; Singleton, Farrer et al. 2003; Chartier-Harlin, Kachergus et al. 2004). The point mutations identified so far include A30P (Kruger, Kuhn et al. 1998), E46K (Zarranz, Alegre et al. 2004), H50Q (Proukakis, Dudzik et al. 2013), G51D (Lesage, Anheim et al. 2013), A53T (Polymeropoulos, Lavedan et al. 1997) and A53E (Pasanen, Myllykangas et al. 2014). In addition to SNCA gene mutations, mutations in other loci like leucinerich repeat kinase-2 (LRRK2), PINK1, PARK 7 are linked with autosomal recessive PD (Goedert, Spillantini et al. 2013).

Although the precise mechanisms and factors affecting PD etiology are not known, a number of perspectives have emerged from clinical studies regarding the disease etiology (Figure 1.5). These viewpoints are often suggested to relate to the putative in vivo function of aSyn. We thus discuss in brief some of the proposed functions along with PD related etiology ascribed to aSyn, as follows:

Endosomal trafficking: Endosomal transport is the crux of neuron signaling. Transport, release and (re)uptake of neurotransmitters occurs at the neuronal synapse through vesicles, which are a part of the endoplasmic reticulum-Golgi complex transport system. aSyn is suggested to bind synaptobrevin- 2 and regulate vesicle fusion at the synapse through the SNARE (soluble N-ethylmaleimide-sensitive factor attachment protein receptor) complex (Burre, Sharma et al. 2010). Over-expression of full length or truncated $\alpha$ Syn in PD mouse model indicated altered SNARE complex localization with aging, coupled with decreased dopaminergic activity (GarciaReitbock, Anichtchik et al. 2010). Moreover, transcriptional profiling in postmortem samples from 26 to 106 years old PD subjects showed consistent down regulation of gene clusters associated with synaptic vesicle transport at ages of over 40 years ( $\mathrm{Lu}$, Pan et al. 2004). The SNARE complex is also required for fusion of late endosomes to lysosomes (Nichols and Pelham 1998). Lysosome based chaperone mediated autophagy (CMA) is one of the main routes for aSyn degradation (Webb, Ravikumar et al. 2003; Cuervo, Stefanis et al. 2004). Thus, disruptions in the endosomal and lysosomal trafficking could be a trigger for accumulation of aSyn in the cytosol, which may result in amyloid formation (Bendor, Logan et al. 2013; Perrett, Alexopoulou et al. 2015). 
aSyn degradation: In addition to enhanced transcription due to gene duplication, faulty degradation of aSyn can be a major source of buildup of excessive protein. $\alpha$ Syn is reported to be degraded by lysosome based CMA as well as the ubiquitin-proteasomesystem (UPS) (Webb, Ravikumar et al. 2003). Studies in live mice indicate that UPS degrades aSyn under normal conditions in vivo but its function is compromised with age and $\alpha$ Syn over-expression. Failure of UPS is suggested to lead to induction of CMA for aSyn degradation (Ebrahimi-Fakhari, Cantuti-Castelvetri et al. 2011). Wt aSyn is proposed to be recognized via the ${ }_{95} \mathrm{VKKDQ}_{99}$ binding motif by hsc70 chaperone. The chaperone- $\alpha$ Syn complex then binds the lysosome membrane receptor (Lamp2) and is internalized for degradation. However, the disease related mutants A30P and A53T are not known to be degraded by CMA possibly due to blocked internalization of hsc70^A30P/A53T aSyn ${ }^{\star}$ Lamp2 complex (Cuervo, Stefanis et al. 2004). Thus, both systems have functional limitations in case of familial and sporadic PD. Moreover, impairment of the UPS initiates a feedback loop of cytosolic protein accumulation and further loss of function (Figure 1.5).

Synaptic plasticity and neurogenesis: The dopaminergic neuronal system is vital for skill learning as it controls the spatial and temporal muscular movements (Clayton and George 1998). Dopamine is also proposed to be vital for proliferation of stem cells in adult neurogenesis and lack of dopamine in PD and LBs pathologies is suggested to be the cause of decreased cognition and depression (Regensburger, Prots et al. 2014). The level of dopamine has been suggested to be related to synuclein expression. Studies in zebra finches have shown elevated level of synelfin (aSyn homolog), up to 15 days post hatching which is the critical period for song development (George, Jin et al. 1995). In humans, classical music performance (requiring refined auditory and motor skills) has been reported to increase the SNCA levels significantly (this is also postulated to be a reflection of the normal response of enhanced dopamine release on listening to music) (Kanduri, Kuusi et al. 2015). Additionally, microarray studies on postmortem tissues suggested a decline in expression of proteins regulating synaptic plasticity with age ( $\mathrm{Lu}$, Pan et al. 2004). Therefore, lack of dopamine, due to aberrant aSyn levels coupled with reduced vesicle transport, can account for some of the symptoms of PD i.e. depression and bradykinesia. 


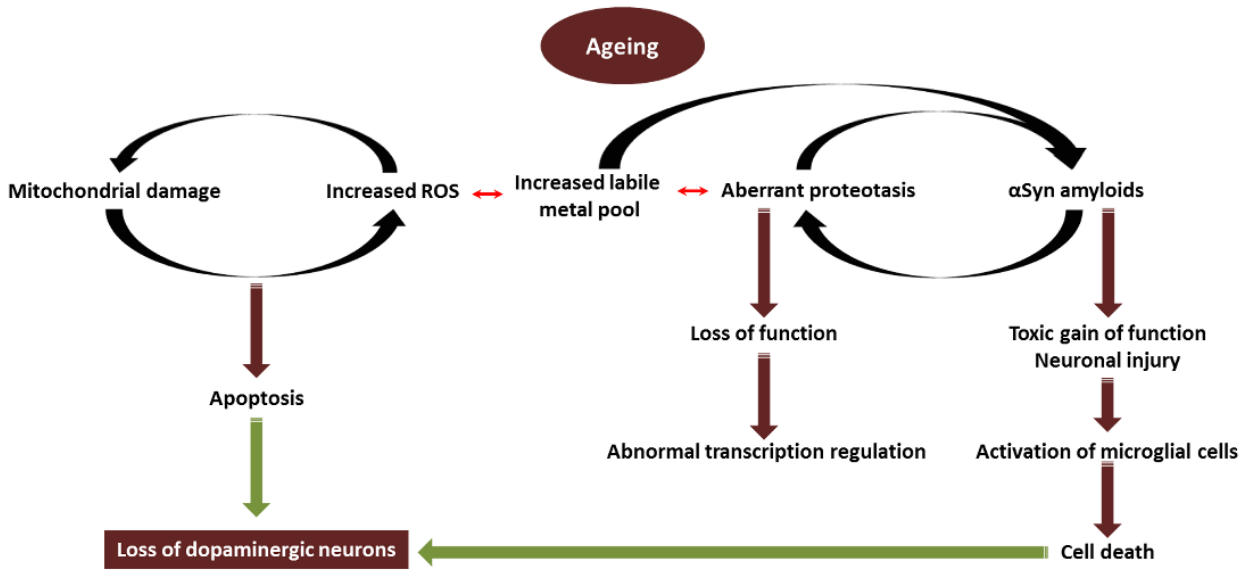

Figure 1.5 Simplistic representation of key cellular functions affected in sporadic PD.

Metal ions-oxidative stress: Trace amounts of metal ions are essential, as cofactors, for functioning of a number of metabolic enzymes and signaling proteins (New 2013). Strict regulation of the amount of metal ions in the brain by the blood brain barrier and accumulation of transition metals like $\mathrm{Fe}^{2+/ 3+}, \mathrm{Cu}^{2+}$ and $\mathrm{Zn}^{2+}$ in neurodegenerative diseases highlights the importance of metal homeostasis for neuronal tissue (Kozlowski, Luczkowski et al. 2012). Toxicity caused by the metal ions in PD and AD is proposed to be due to two mechanisms. Firstly, the excess of redox active metals ions like $\mathrm{Fe}^{2+/ 3+}$ and $\mathrm{Cu}^{2+}$ can participate in Fenton reactions producing reactive oxygen species (ROS): hydroxyl radicals. Although $\mathrm{Zn}^{2+}$ is a redox inert metal ion and does not form ROS, based on the Irving-William series it can displace other metal ions like $\mathrm{Co}^{2+}, \mathrm{Ni}^{2+}, \mathrm{Fe}^{2+}$ from proteins and generate a labile metal pool (Gorelsky, Basumallick et al. 2005; New 2013). The Fenton reaction based ROS generation initiates a positive-feed-forward loop, recycling the redox active metal ion while producing ROS. The highly reactive hydroxyl radical can react with proteins, like $A \beta$ and $\alpha$ Syn, and with nucleotides disrupting proteostasis and leading to cell death (Cuajungco, Goldstein et al. 2000; Passos and Von Zglinicki 2006).

Secondly, the free metal ions might interact with soluble proteins like aSyn and induce their aggregation into oligomeric and/or fibrillar forms (Bush, Pettingell et al. 1994; Breydo, Wu et al. 2012; Viles 2012). Aggregation of proteins leads to loss of function, thus affecting proteostasis. The substantia nigra neurons that degenerate in 
PD contain neuromelanin pigment. The pigment is suggested to increase from the age of 1-2 years to about 60 years. Functionally, neuromelanin has been associated with sequestration of a range of organic compounds and redox active metal ions capable of producing ROS (Zecca, Tampellini et al. 2001). Thus, it is believed to be neuroprotective, however neuromelanin itself is understood to be susceptible to ROS. With age, it is proposed that neuromelanin becomes saturated in its chelation property, which possibly makes it vulnerable to degradation because of cytoplasmic ROS. As a result the sequestered toxins may be released, leading to neurotoxicity and cell death (Zareba, Bober et al. 1995).

Mitochondrial dysfunction: Mitochondria are the hubs of redox reactions. A number of enzymes along the electron transport chain (ETC) generate and sequester ROS (Kowaltowski, de Souza-Pinto et al. 2009). Errors in the balance between ROS generation and sequestration are recognized as a natural part of aging. Consequently increased rate of mitochondrial DNA mutations, defective ETC, mitophagy and apoptosis are often observed in clinical and transgenic animal studies of age associated neurodegenerative diseases like PD, AD and dementia (Lu, Pan et al. 2004; Reddy, McWeeney et al. 2004; Lin and Beal 2006; Trifunovic 2006). In idiopathic PD, genetic mutations in Parkin, PINK1 and DJ-1 are associated with mitochondrial dysfunction (Goedert, Spillantini et al. 2013). In addition, a genetic mutation linked to familial PD, A53T aSyn, is indicated to accelerate mitochondrial impairment (Devi, Raghavendran et al. 2008). Studies on aSyn-membrane interactions suggest that aSyn may act as a shuttle on mitochondrial membranes and repair peroxidized lipids via methionine sulfoxide reductase A (MsrA) enzyme (Maltsev, Chen et al. 2013). Therefore, in healthy cells aSyn may have a role in mitochondrial function, which is most likely altered in pathogenesis.

\section{Structure of $\alpha$ Syn}

Monomers: Monomeric aSyn is believed to be an intrinsically disordered protein and is anticipated to gain structure in the cellular context of protein-protein interactions (Eliezer, Kutluay et al. 2001; Uversky 2011; Deleersnijder, Gerard et al. 2013). However, some recent but contested reports suggest aSyn to have a helical tetrameric and multimeric organization in vivo in red blood cells and brain tissue (Bartels, Choi et al. 2011; Luth, Bartels et al. 2015). At primary level, $\alpha$ Syn is made of 140 amino acids and 
has imperfect repeats of 11 amino acids in the N-terminus. Each of these imperfect repeats has a conserved hexameric motif of KTKEGV. This motif is also found in the amphipathic $\alpha$-helices of apolipoproteins, thus suggesting a lipid binding function (Davidson, Jonas et al. 1998) (Figure 1.6). Threonines in six of the imperfect repeats (position: T22, T33, T44, T59, T81, T92) are purported to be the curvature sensing residues that enable binding to acidic and highly curved membranes (Pranke, Morello et al. 2011). The central region of the protein, residue 61 to 95, is known as NAC region (non $\mathrm{A} \beta$ amyloid component). It has 3 imperfect KTKEGV repeats along with a high density of hydrophobic amino acids. This hydrophobic patch in the center of the $\alpha$ Syn sequence is shown to be critical for its amyloidogenic properties, as $\beta$ Syn lacking this region is not known to form amyloids (Yamin, Munishkina et al. 2005). In aggregated form, the NAC region is believed to form the core of the cross $\beta$-sheet structure. In addition, point mutations in this region are reported to hamper $\alpha$ Syn aggregation (Giasson, Murray et al. 2001). The C-terminus of aSyn is rich in acidic (aspartic and glutamic acid) and proline residues. It is understood to be unstructured both when bound to lipid molecules as well as when assembled into fibrils (Davidson, Jonas et al. 1998; Del Mar, Greenbaum et al. 2005). Due to the high negative charge density at physiological $\mathrm{pH}$, this region is important for electrostatic interactions of the monomers as well as of the amyloid structures (Murray, Giasson et al. 2003; Hoyer, Cherny et al. 2004; Sweers, van der Werf et al. 2012). Though no defined secondary or tertiary structure is reported in recombinantly produced $\alpha$ Syn, long range interactions between the residues 120-140 in the C-terminus and 30-100 in the center indicate the presence of intramolecular interfaces (Dedmon, Lindorff-Larsen et al. 2005).

The N-terminus of aSyn adopts an $\alpha$-helical conformation in the presence of SDS micelles and lipid vesicles (Davidson, Jonas et al. 1998; Eliezer, Kutluay et al. 2001; Georgieva, Ramlall et al. 2008) (Figure 1.6). This structural transition is believed to embody its putative in vivo role of binding to synaptic vesicles (Snead and Eliezer 2014). aSyn is reported to bind lipid vesicles in an extended conformation, where residue 1-100 form an amphipathic $\alpha$-helix, and a broken helix conformation, where the continuity of the helix is interrupted by a linker region between residue 42-44 or 37-45 (Bussell and Eliezer 2003; Ulmer, Bax et al. 2005; Georgieva, Ramlall et al. 2008; Trexler and Rhoades 2009). The conformation adopted by aSyn molecules appears to be influenced by the curvature of the membrane, with high curvature favoring the broken helix 


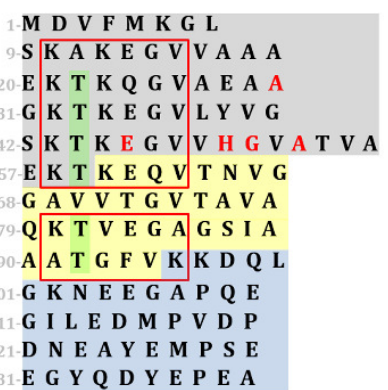

A

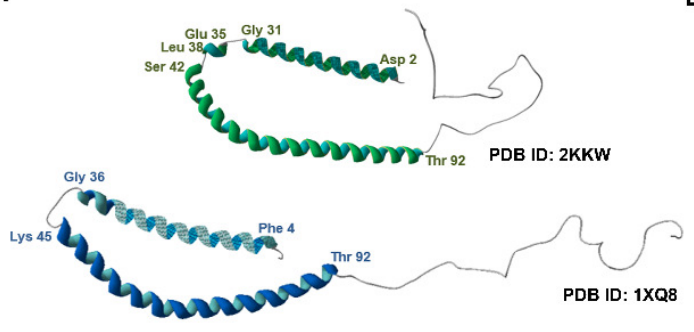

Figure 1.6 Structural features of monomeric aSyn. (A) Amino acid sequence arranged to show 7 hexameric imperfect repeats of K-T-K-[E/Q]-[G/Q]-V in red squares, 6 threonines critical for curvature sensing highlighted in green, residues mutated in early onset of PD in red, $\mathrm{N}$-terminal region highlighted in grey, NAC region highlighted in yellow and C-terminal region highlighted in blue. (B) $\alpha$-helical structure of monomer when bound to SLAS (PDB ID: 2KKW) and SDS (PDB ID: 1XQ8) micelle. The figures were prepared from the structural data of PDB ID: 2KKW and 1XQ8 using SPDBV $\mathrm{v} 4.10$.

conformation and low curvature supporting an extended conformation (Trexler and Rhoades 2009). Besides the curvature of the membrane, the charge on the lipids and residue specific modifications like phosphorylation, acetylation, nitration, ubiquitination, methionine oxidation, sumolyation on aSyn molecule decisively affect aSyn-membrane interaction (Snead and Eliezer 2014).

Few studies report tertiary structure of aSyn in complex with other molecules, and no structure is available for aSyn alone. Search in the PDB as of 23 September 2015, showed 16 entries for structure on human aSyn (UniProtKB ID: P37840) determined by NMR, EPR, electron crystallography: micro-electron diffraction (microED) and Xray crystallography (Table 1.4). These studies show that the N-terminal domain of aSyn interacts with micelles and membrane proteins adopting an $\alpha$-helical fold and that the number of participating residues is highly context dependent (Ulmer, Bax et al. 2005; Rao, Jao et al. 2010; Zhao, Cascio et al. 2011). 
Table 1.4 List of available aSyn structures from Homo sapiens (UniprotKB ID: P37840) (Till 23 September 2015).

\begin{tabular}{|c|c|c|c|c|c|}
\hline $\begin{array}{l}\text { PDB } \\
\text { ID }\end{array}$ & Method & Resol. & aSyn residues & Ligand & Ref. \\
\hline $1 X Q 8$ & Soln NMR & NA & $1-140$ & SDS micelle & $\begin{array}{c}\text { (Ulmer, Bax et al. } \\
\text { 2005) }\end{array}$ \\
\hline 2JN5 & Soln NMR & NA & $1-12$ & $\begin{array}{c}\text { Synphilin-1: } 349-557 \\
\text { aa }\end{array}$ & (Xie, Zhou et al. 2010) \\
\hline $2 K K W$ & $\begin{array}{c}\text { EPR, } \\
\text { Soln NMR }\end{array}$ & NA & 140 & SLAS-micelle & (Rao, Jao et al. 2010) \\
\hline $2 X 6 M$ & X-Ray & $1.62 \AA$ & $132-140$ & $\begin{array}{l}\text { Heavy chain variable } \\
\text { domain: camelid } \\
\text { antibody (Camelus } \\
\text { dromedarius) } 126 \mathrm{aa}\end{array}$ & $\begin{array}{c}\text { (De Genst, Guilliams } \\
\text { et al. 2010) }\end{array}$ \\
\hline $3 Q 25$ & X-Ray & $1.90 \AA$ & $\begin{array}{l}\text { 1-19 fused to } \\
\text { MBP }\end{array}$ & $\begin{array}{l}\text { Glycerol, Maltose, } \\
\text { Sulfate ion }\end{array}$ & $\begin{array}{l}\text { (Zhao, Cascio et al. } \\
\text { 2011) }\end{array}$ \\
\hline $3 Q 26$ & X-Ray & $1.54 \AA$ & $\begin{array}{c}\text { 10-42 fused to } \\
\text { MBP }\end{array}$ & $\begin{array}{l}\text { Glycerol, Maltose, } \\
\text { Sulfate ion }\end{array}$ & $\begin{array}{c}\text { (Zhao, Cascio et al. } \\
\text { 2011) }\end{array}$ \\
\hline $3 Q 27$ & X-Ray & $1.30 \AA$ & $\begin{array}{c}32-57 \text { fused to } \\
\text { MBP }\end{array}$ & $\begin{array}{l}\text { Glycerol, Maltose, } \\
\text { Sulfate ion }\end{array}$ & $\begin{array}{c}\text { (Zhao, Cascio et al. } \\
\text { 2011) }\end{array}$ \\
\hline $3 Q 28$ & X-Ray & $1.60 \AA$ & $\begin{array}{c}\text { 58-79 fused to } \\
\text { MBP }\end{array}$ & Maltose, Sulfate ion & $\begin{array}{c}\text { (Zhao, Cascio et al. } \\
\text { 2011) }\end{array}$ \\
\hline $3 Q 29$ & $\mathrm{X}$-Ray & $2.30 \AA$ & $\begin{array}{l}\text { 1-19 fused to } \\
\text { MBP }\end{array}$ & $\begin{array}{l}\text { Glycerol, Maltose, } \\
\text { Sulfate ion }\end{array}$ & $\begin{array}{c}\text { (Zhao, Cascio et al. } \\
\text { 2011) }\end{array}$ \\
\hline $2 M 55$ & Soln NMR & NA & $\begin{array}{c}\mathrm{N} \text {-term } \\
\text { acetylated 1-19 }\end{array}$ & $\begin{array}{l}\text { Calmodulin (Homo } \\
\text { sapiens) } 148 \text { aa }\end{array}$ & $\begin{array}{c}\text { (Gruschus, Yap et al. } \\
\text { 2013) }\end{array}$ \\
\hline $4 B X L$ & Soln NMR & NA & $35-56$ & $\begin{array}{l}\text { Engineered binding } \\
\text { protein: AS69 } \\
\text { (synthetic) } 46 \text { aa }\end{array}$ & $\begin{array}{c}\text { (Mirecka, } \\
\text { Shaykhalishahi et al. } \\
\text { 2014) }\end{array}$ \\
\hline $4 R 0 U$ & X-Ray & $1.38 \AA$ & $72-78$ & None & (Li, Jones et al. 2014) \\
\hline $4 R 0 W$ & X-Ray & $1.50 \AA$ & $70-76$ & None & (Li, Jones et al. 2014) \\
\hline $4 R I K$ & X-Ray & $1.85 \AA$ & $69-77$ & None & $\begin{array}{c}\text { (Rodriguez, Ivanova et } \\
\text { al. 2015) }\end{array}$ \\
\hline $4 R I L$ & microED & NA & $68-78$ & None & $\begin{array}{c}\text { (Rodriguez, Ivanova et } \\
\text { al. 2015) }\end{array}$ \\
\hline $4 Z N N$ & microED & NA & $47-56$ & None & $\begin{array}{l}\text { (Rodriguez, Ivanova et } \\
\text { al. 2015) }\end{array}$ \\
\hline
\end{tabular}

Soln: Solution, NA: Not available, Resol.: resolution. 
Oligomers: aSyn oligomers formed during aggregation can be transient as well as stable (Lorenzen, Nielsen et al. 2014; Paslawski, Mysling et al. 2014). Independent studies based on analytical ultracentrifugation, single-molecule photo-bleaching and size exclusion chromatography coupled techniques characterize two discrete oligomer populations in aSyn. The two populations of oligomers are proposed to consist of 18 and 29 monomers (Chen, Drakulic et al. 2015), 15-19 and 34-38 monomers (Zijlstra, Claessens et al. 2014) and about 30 monomers (for small oligomers; number for large oligomers is not available) (Lorenzen, Nielsen et al. 2014) per oligomer. The oligomers are reported to have $35 \pm 5 \% \beta$-sheet structure in comparison to $65 \pm 10 \%$ for fibrils (Chen, Drakulic et al. 2015). In shape, $\alpha$ Syn oligomers are described as annular (with a pore in the center) and tubular (elongated; without pore) by AFM, EM, SAXS studies (Lashuel, Petre et al. 2002; Giehm, Svergun et al. 2011; Chen, Drakulic et al. 2015).

Fibrils: On aggregation, the intrinsically disordered aSyn is known to adopt the canonical amyloid structure with cross $\beta$-sheets (Serpell, Berriman et al. 2000). The $\beta$ sheets are arranged into multiple parallel, in register strands, running perpendicular to the fibril axis, with an intermolecular $\beta$-strand distance of 0.47-0.48 nm (Chen, Margittai et al. 2007). Solid-state NMR (ssNMR) studies on aSyn fibrils reveal distinct $\beta$-strands in the fibril core, but the exact residues adopting $\beta$-sheet fold varies between studies (Heise, Hoyer et al. 2005; Chen, Margittai et al. 2007; Vilar, Chou et al. 2008; Gath, Bousset et al. 2014). Apart from the study by Gath et al., where the N-terminal residues were reported to acquire $\beta$-sheet structure, all other studies show residue 1-30 (N-terminus) and 110-140 (C-terminus) to be mobile i.e. they are not involved in $\beta$ sheet formation (Table 1.5). Experiments based on proteinase $\mathrm{K}$ digestion have also shown susceptibility of the $\mathrm{N}$ and $\mathrm{C}$ termini to digestion, emphasizing that these regions are labile and most probably not in the structural core of the fibrils (Conway, Harper et al. 2000; Miake, Mizusawa et al. 2002; Qin, Hu et al. 2007). The aforementioned ssNMR studies determine the residues specific secondary structure, of a $\beta$-fold, based on experimentally calculated torsional angles. No crystal or NMR structure for $\alpha$ Syn fibrils is available. 
Table 1.5 List of residues adopting $\beta$-fold in $\alpha$ Syn fibrils.

\begin{tabular}{|c|c|c|c|c|c|c|c|c|c|}
\hline \multicolumn{8}{|c|}{ Residues adopting $\beta$-fold } & \multirow{2}{*}{$\begin{array}{c}\text { Sample } \\
\text { A }\end{array}$} & \multirow{2}{*}{$\begin{array}{c}\text { Ref. } \\
\text { (Heise, Hoyer et } \\
\text { al. 2005) }\end{array}$} \\
\hline $38-42$ & $52-57$ & $61-65$ & $69-72$ & $74-82$ & $87-92$ & 94-95 & & & \\
\hline 42 & $53-57$ & $61-62$ & $64-66$ & 75-79 & $87-88$ & $90-92$ & & B & $\begin{array}{l}\text { (Heise, Hoyer et } \\
\text { al. 2005) }\end{array}$ \\
\hline $35-40$ & $51-54$ & $69-87$ & $95-98$ & & & & & & $\begin{array}{c}\text { (Chen, } \\
\text { Margittai et al. } \\
\text { 2007) }\end{array}$ \\
\hline $35-41$ & $49-56$ & $60-67$ & $69-82$ & $86-94$ & & & & $\begin{array}{l}\text { Ext. slow } \\
\text { exchange }\end{array}$ & $\begin{array}{c}\text { (Vilar, Chou et } \\
\text { al. 2008) }\end{array}$ \\
\hline $39-44$ & $49-56$ & $62-68$ & $70-83$ & $90-96$ & & & & $\begin{array}{c}\text { Slow } \\
\text { exchange }\end{array}$ & $\begin{array}{c}\text { (Vilar, Chou et } \\
\text { al. 2008) }\end{array}$ \\
\hline $16-20$ & $38-44$ & $46-50$ & $52-56$ & 61-66 & $68-72$ & 75-79 & $84-92$ & Fibrils & $\begin{array}{l}\text { (Gath, Bousset } \\
\text { et al. 2014) }\end{array}$ \\
\hline $1-6$ & $9-12$ & $15-19$ & $23-30$ & $34-43$ & $58-66$ & $69-79$ & $87-97$ & Ribbons & $\begin{array}{l}\text { (Gath, Bousset } \\
\text { et al. 2014) }\end{array}$ \\
\hline
\end{tabular}

Ext.: extremely

\section{Polymorphism in $\alpha$ Syn and prion-like character?}

aSyn fibrils like most amyloid fibrils show polymorphism (Figure 1.7). Discrete morphologies observed in the fibrils are reported to be influenced by the aggregation conditions (Hoyer, Antony et al. 2002; Heise, Hoyer et al. 2005; Vilar, Chou et al. 2008; Bousset, Pieri et al. 2013). Furthermore, ssNMR studies suggest that each polymorph, as observed in EM studies, is associated with specific structural features (Heise, Hoyer et al. 2005; Vilar, Chou et al. 2008; Gath, Bousset et al. 2014). Based on these structural studies, the main differences between polymorphs could be due to one or more of the following parameters:

- The residues involved in $\beta$-sheet formations, including a quantitative and/or a qualitative difference (Table 1.5).

- Differences in the conformation of the monomers (intramolecular contacts).

- Differences in the intermolecular contacts.

Thus, these fibrils with typical structures seem organizationally different from each other. This could mean that aSyn fibrils with different structure and morphology have possibly disparate biochemistry as well. Indeed, studies comparing the neurotoxicity of polymorphs advocate that the polymorphs, referred to as strains of aSyn, have distinct 
seeding and propagation properties in vitro, in vivo as well as in animal models of $\mathrm{PD}$ (Bousset, Pieri et al. 2013; Narkiewicz, Giachin et al. 2014).

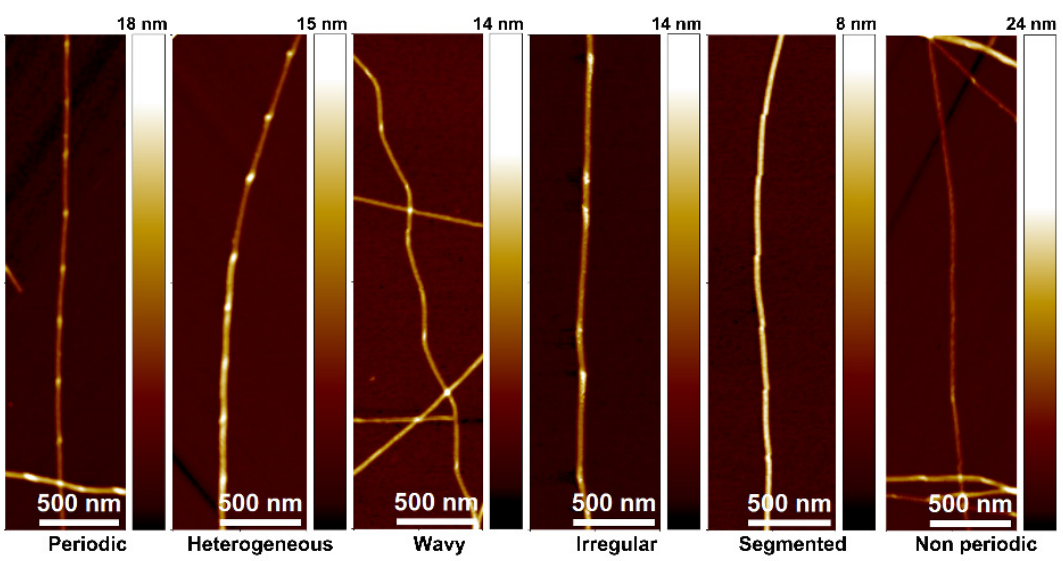

Figure 1.7 AFM height images illustrating structural polymorphism observed in A53T aSyn fibrils prepared in $10 \mathrm{mM}$ Tris- $\mathrm{HCl}, 10 \mathrm{mM} \mathrm{NaCl}$ with $0.1 \mathrm{mM}$ EDTA at $\mathrm{pH}$ 7.4.

In recent years, the hypothesis over the pattern of origin of initial aSyn aggregates, possibly leading to PD has been revisited. Earlier, aberrations in protein degradation mechanism with age were postulated as the chief cause of aSyn accumulation and aggregation in the cell (in idiopathic PD). Accordingly, the process of aggregation and ensuing toxicity was presumed to be a cell level phenomenon, occurring independently in affected cells. However, mounting experimental evidence suggests that the initial aggregation event takes place in a small number of cells, sporadically. Thereon the aggregated assemblies, oligomers and/or fibrils, can transfer to the neighboring cells and spread the pathology. This cell-to-cell transmission of aggregates is the basis for the Braak staging for PD and bears resemblance to the prion-like spread for PD (Dunning, George et al. 2013; George, Rey et al. 2013; Goedert, Spillantini et al. 2013; Goedert, Falcon et al. 2014). Hence increasing number of studies are investigating the putative prion-like nature of aSyn amyloids. So far, results indicate aSyn to have following prion-like characteristics:

Nucleation and templating: Like prions, amyloid formation in aSyn is believed to initiate through formation of a critical nucleus (Wood, Wypych et al. 1999; Buell, Galvagnion et al. 2014; Narkiewicz, Giachin et al. 2014). It is then, assumed to recruit 
the unfolded soluble monomers on the ends and elongate into fibrils (Buell, Galvagnion et al. 2014). Furthermore, the nucleus or the seed (pre-formed fibril) is suggested to act as a template for the incorporating monomer (Bousset, Pieri et al. 2013).

Strains: Similar to prion strains, the polymorphs are stated to promote propagation of their conformation by seeding. In addition, each polymorph is proposed to exhibit a distinct toxicity profile, a feature suggested to be equivalent to strong and weak phenotypes of prions (Bousset, Pieri et al. 2013; Goedert, Falcon et al. 2014; Narkiewicz, Giachin et al. 2014; Peelaerts, Bousset et al. 2015).

Cell-to-cell transfer: Numerous cell and animal-based reports reveal that aSyn aggregates can undergo inter-neuronal transfer. The transfer is accompanied by aggregation of the endogenous aSyn (similar to corruption of native protein conformation, in case of prions), resulting in neurotoxicity and PD like symptoms (Dunning, George et al. 2013; George, Rey et al. 2013; Watts, Giles et al. 2013; Goedert, Falcon et al. 2014; Holmqvist, Chutna et al. 2014; Peelaerts, Bousset et al. 2015).

Cross seeding: In addition to templating aggregation of soluble proteins of the same sequence (homologous seeding), prions and amyloid proteins have been shown to induce misfolding of heterologous protein sequences (Prusiner 1998; Kotzbauer, Giasson et al. 2004; Krebs, Morozova-Roche et al. 2004; Vanik, Surewicz et al. 2004). Initially, transmission of prion pathology across species was believed to be restricted due to species barrier (Prusiner 1998). The main determinant of the species barrier was proposed to be the primary sequence similarity (homology) between the prion proteins. However, observation of strain specific cross seeding between yeast models and sporadic cross seeding of heterologous (low sequence similarity) proteins highlight the critical role of prion conformation in interspecies transmission (Collinge 2001; Vanik, Surewicz et al. 2004; Tanaka, Chien et al. 2005). In recent years, aSyn has been shown to cross seed aggregations in a sequence as well as conformation specific manner (Giasson, Forman et al. 2003; Kotzbauer, Giasson et al. 2004; Yonetani, Nonaka et al. 2009). Moreover, strain dependent sensitivity of cross seeding between aSyn and tau shows the importance of subtle conformational differences in spreading of amyloid conformation (Guo, Covell et al. 2013).

Hence, aSyn does appear to show some key features associated with prions' generation and propagation. Besides, it also seems to share some of the unknowns linked to prions, for example, precise function of the native protein in the cell, triggers 
for the toxic gain of function, mode of toxicity, and principle toxic species. However, unlike prions, aSyn is not known to be infective between organisms, which is a major qualifier that makes aSyn prion-like and not a prion.

\section{MICROSCOPY TECHNIQUES USED FOR VISUALIZATION OF $\alpha$ SYN AMYLOIDS}

A major proportion of this thesis concerns single fibril level studies of aSyn fibrils. AFM and STEM were mainly used for high resolution fibril imaging. In the following sections we briefly discuss the general working principles of these microscopic techniques.

\section{Atomic force microscopy}

Atomic force microscopy is an important imaging technique for studying morphological and mechanical properties of amyloids at a resolution of a few nanometers (Khurana, Ionescu-Zanetti et al. 2003; Smith, Knowles et al. 2006; van Raaij, Segers-Nolten et al. 2006; Adamcik, Jung et al. 2010). AFM produces an image by scanning the surface of a specimen using a nanoscale sharp tip. The tip is positioned at the end of a flexible cantilever (Figure 1.8A). The tip and the cantilever are usually made of silicon nitride and can have variable lengths, spring constants and resonance frequencies (Alessandrini and Facci 2005; Morris, Kirby et al. 2010). A number of tip parameters like sharpness, shape, stiffness, etc. are important for the quality of imaging. The sharpness of the tip, expressed as the tip radius, is one of the factors determining the scanning resolution. The shape of the tip, described as the aspect ratio, delimits the penetration depth of the tip, which affects the image resolution due to probebroadening or tip-convolution (van Raaij, Segers-Nolten et al. 2006). A tip of appropriate stiffness ensures optimal tip-sample interaction (without deformation) for high signal to noise ratio. The choice of tip depends on the imaging mode as well on the experiment. For specialized experiments, tips can be modified in a number of ways like coating with a conductive layer of $\mathrm{Au}$ or $\mathrm{Pt}$ for conductivity measurements, functionalization with specific ligands for studying protein-protein interactions, or tips of special geometry for force experiments (Alessandrini and Facci 2005). The working of an AFM can be described in two steps, sample scanning by the tip and detection of the tip movement to generate an image. 

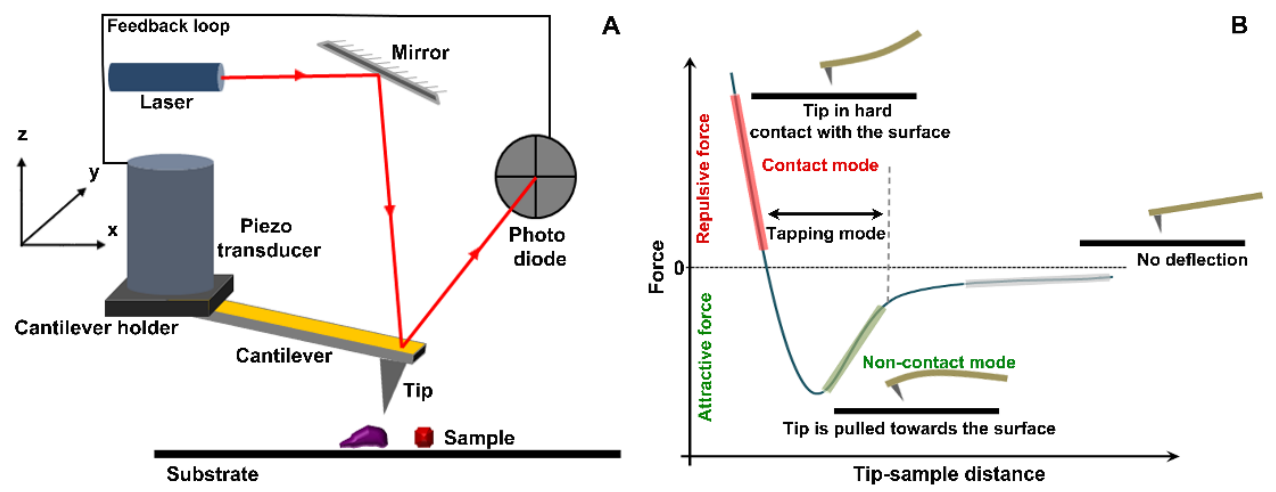

Figure 1.8 (A) Simplistic representation of tapping mode AFM with a movable tip in $x, y, z$ directions. (B) A standard force distance curve.

\section{Sample scanning}

The sample to be imaged is typically adsorbed on an atomically flat surface like mica. The cantilever is positioned over the area of interest and the tip is engaged to the surface. The scanning can be performed in three arrangements (1) fixed sample stage and a movable tip $(x, y, z),(2)$ a movable sample stage $(x, y, z)$ and a fixed tip, (3) a movable scanner $(x, y)$ and a tip moving in the $z$-direction (used in this thesis). In all scenarios, the movement of the tip and/or the sample is controlled by piezoelectric transducers. The piezoelectric transducers contract or expand on applying a bias voltage with a subAngstrom sensitivity. Thus, regulating the bias voltage, the movement of the piezoelectric transducers can be manoeuvred in three orthogonal directions, $x, y$ and $z$ with precision of nanometers. To produce an image the sample is scanned line by line usually in a raster fashion. During scanning the tip interacts with the sample and the quantitative and the qualitative characteristics of this interaction depend on the mode of scanning (Alessandrini and Facci 2005; Morris, Kirby et al. 2010). Two key modes for scanning include, the $D C$ mode and the $A C$ mode (the mode used in this thesis):

$D C$ mode or Contact mode: in this mode the tip is in contact with the sample while scanning. Contact mode scanning can be performed in air or in liquid using a pre-set imaging force. The imaging force is kept constant by a feedback loop that maintains a constant cantilever deflection (set-point) by vertically moving the scanner at each $x, y$ scan step. The force exerted on the sample in contact mode is also determined by the 
spring constant of the cantilever. For contact mode, usually triangular or $\mathrm{V}$ shaped cantilevers with low spring constant of $0.01-1.0 \mathrm{~N} . \mathrm{m}^{-1}$ and a resonance frequency of 7$50 \mathrm{kHz}$ are recommended (Morris, Kirby et al. 2010). The force experienced by the tip on scanning the sample in this mode is repulsive in nature force (Figure 1.8B). In addition to the repulsive van der Waals force the tip also experiences frictional (resistive force) and capillary forces (attractive force). The capillary forces can be overcome by imaging in liquid or in an enclosed setup with very low humidity. Due to relatively high force exerted on the sample, contact mode is not always suitable for delicate biological samples.

$A C$ mode: in this mode the tip is not in direct contact with the sample as the cantilever is oscillated near its resonance frequency $\left(\omega_{0}\right)$ above the sample. The oscillating cantilever experiences long range attractive forces during the tip-sample interaction. Depending on the extent of contact with the sample there are two types of AC mode imaging, tapping mode and true non-contact mode. In this thesis all AFM imaging has been done in tapping mode, therefore we describe only this mode in more detail.

Tapping mode: in tapping mode the cantilever is oscillated at an amplitude of about $100 \mathrm{~nm}$ near the sample surface producing intermittent contact with the sample. The tapping amplitude as well as the amplitude set point ratio determine the image quality in tapping mode AFM. The amplitude of the oscillations should be large enough to overcome the capillary forces yet mild enough to gently tap on the sample surface without deforming the sample. A tip tuned to its resonance frequency $\left(\omega_{0}\right)$ oscillates at a free amplitude $\left(A_{o}\right)$. Engaging the tip to the surface dampens the free amplitude, due to tip-sample interaction, to the tapping amplitude $(A)$. The ratio between the tapping and the free amplitude is defined as the set point ratio $\left(s=A / A_{o}\right)$ and is maintained by the feedback loop to ensure constant tapping force on the sample while imaging (Legleiter 2009). For tapping mode, stiffer cantilevers of 30-60 N.m ${ }^{-1}$ with a resonance frequency of 250-350 kHz are ideal (Morris, Kirby et al. 2010).

\section{Detection}

Movement of the tip while it scans the sample can be detected using optical or electrical methods. Optical methods for detection include, laser beam deflection and interferometry. Electrical detection methods include, electron tunnelling, capacitance 
and piezoelectric cantilevers (Morris, Kirby et al. 2010). Laser beam deflection is the most commonly used detection method. In this method, the tip movement is followed by tracking the displacement of a laser beam that is focused on the reflective surface of the cantilever (Figure 1.8A). The reflected beam from the cantilever surface is positioned at the center of a split photodiode detector. As the tip scans over a sample surface the topographical features of the sample deflect the tip. Deflection of the tip is registered as displacement of the laser beam in one of the quadrants of the photodetector. Changes in the laser beam position in the quadrant provides information about the sample topography, lateral movement and twisting of the tip. A feedback loop adjusts the voltage to the piezo to restore the pre-defined set point. The resulting movement of the piezo is recorded to produce an image.

In this thesis the morphological features of the aSyn amyloids, the height and the periodicity, have been studied using tapping mode AFM in air (Bioscope Catalyst, Bruker). The height and periodicity analyses of the fibrils were carried out using a custom written Matlab Script employing DIPimage toolbox (version 2.3, TU Delft, Delft, The Netherlands) (Faas, Rieger et al. 2009). For height and periodicity measurement, the AFM images were first plane corrected using line by line levelling. Next, the fibril of interest was traced by specifying two points on the fibrils. The two points identify the length of the fibril to be analyzed.

For height determination, the highest pixels over the length of the fibril were traced (between the two points). The fibril height is the average height of the pixels along the traced line. Tracing of a periodic fibrils between two points of interest results in a longitudinal profile of the fibril that shows consecutive peaks and troughs (Figure 1.9B). The periodicity of the fibrils is determined by the frequency of the peaks using Fourier transformation.

In this regime the determined average height of a fibril can be influenced by the periodicity of the fibril. In fibrils with same length but different periodicities i.e. 110 and $360 \mathrm{~nm}$, the ratio of pixels representing peak and trough height is different for a given length. In fibrils of larger periodicities the relative number of pixels representing peak height is less than the number of pixels representing trough heights, thus the average height of such fibrils is shifted towards the trough heights (Figure 1.9). 

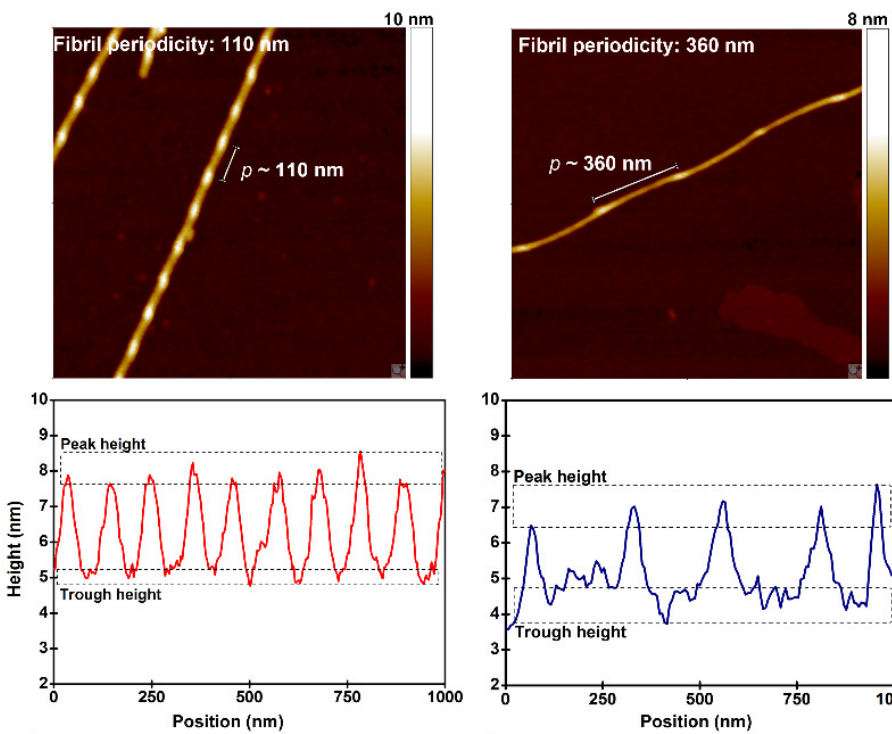

A
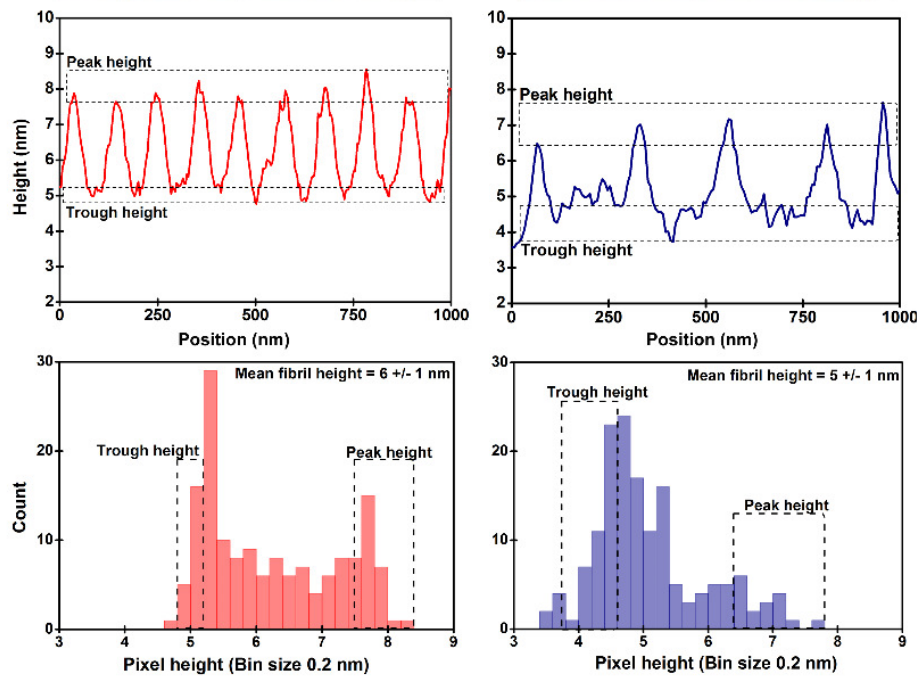

B

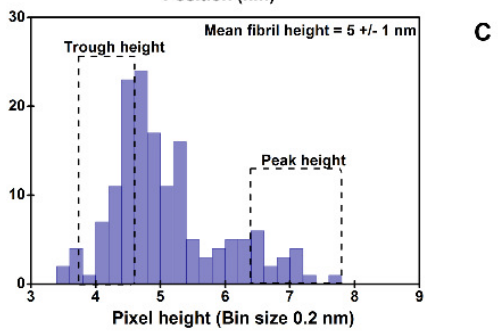

Figure 1.9 Panel (A) Typical AFM height images used for morphology analysis, showing period length represented as " $p$ ". The fibril is traced longitudinally to yield a profile as in panel (B) showing peaks and troughs. A total of 155 pixels were counted for $1 \mu \mathrm{m}$ length of fibrils in this example. The pixel height is plotted in a histogram as in panel (C). Image size: $1 \times 1 \mu \mathrm{m}$ (fibril periodicity $110 \mathrm{~nm}$ ) and $1.5 \times 1.5 \mu \mathrm{m}$ (fibril periodicity $360 \mathrm{~nm}$ ).

\section{Scanning transmission electron microscopy}

Scanning transmission electron microscopy provides a combination of transmission electron microscopy (TEM) and scanning electron microscopy (SEM). STEM works on the same principle as SEM, where a focused beam of electrons scans the sample to produce images. However, unlike SEM, thin samples are used to allow transmission of electrons. The transmitted electrons allow higher signal and enhanced spatial resolution (Pennycook, Lupini et al. 2007). 
The key modules of a STEM include the electron column, scanning system coils and detectors (Figure 1.10A). The first component of an electron column is an electron gun that generates an electron beam. The common sources for the electron beam include tungsten and lanthanum hexaboride $\left(\mathrm{LaB}_{6}\right)$ filament cathodes. The energy, current and the size of the electron beam depends on the beam source and determines the resolution of the imaging. Typically, the produced electron beam in a STEM has energies ranging from 1-40 keV. The second component of the electron column is a series of two or more magnetic lenses that focus the beam into a narrow probe of a few nanometers diameter. Next, the focussed beam passes through the scanning coils that manoeuvres the beam and enables scanning of the sample in a raster fashion. On hitting the sample the focused electron beam penetrates to a certain depth and produces elastic and inelastic scattering of the sample electrons (on the surface as well as in the bulk). The volume of the sample influenced by the beam-sample interaction is named as the interaction volume.

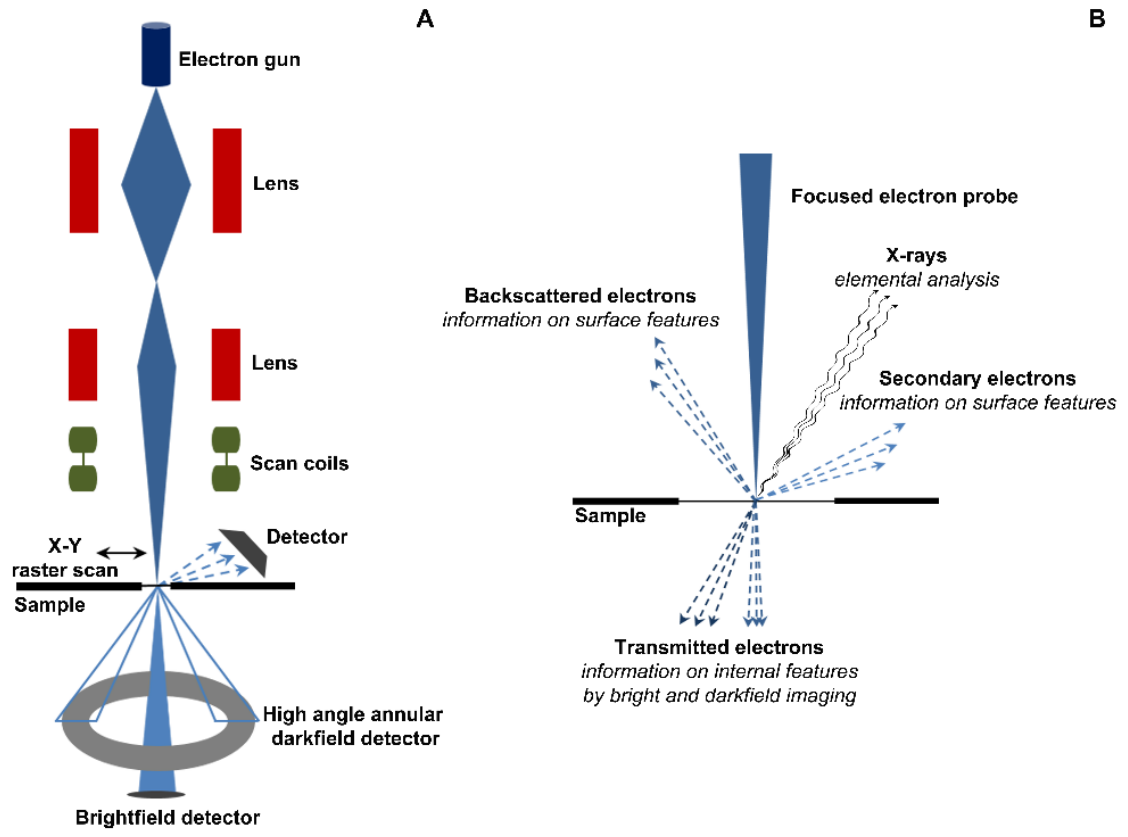

Figure 1.10 (A) Simple schematic representation of a STEM. (B) Various types of electrons produced in beam-sample interaction. 
The interaction volume is determined by the penetration depth of the electron beam, which is dependent on the energy of the beam and the atomic constitution of the sample. The electrons emitted and transmitted from the sample by the incident electron beam are used to produce the STEM image (Figure 1.10B). The electrons generated are grouped in the following categories (Liu 2005; Pennycook, Lupini et al. 2007):

Backscattered electrons: The impact of the incident electron beam can produce elastic scattering on the surface of the sample. The scattered electrons can leave the sample surface and form the back scattered electron (BSE) signal. Samples with higher atomic number elements produce more BSE than low atomic number elements and consequently display a higher contrast. The BSE signal thus provides information about the surface features and chemical composition of the sample.

Secondary electrons: The energy from the incident electron beam can also knockout electrons from the $\mathrm{k}$-shell of the sample atoms by inelastic scattering. These electrons are named as secondary electrons (SE). This type of beam-sample interaction is dominant on the sample surface. The amount of SE produced depends on the angle of incidence and thus allows contrast between topographical features of the sample.

Transmitted electrons: In thin samples the incident electrons can pass through the sample. Transmission can occur at comparably large angles relative to the optical axis after scattering through the sample or at small angles with minor scattering. The electrons scattered at large angles can be detected by a high angle annular darkfield detector (HAADF). The special annular geometry of the detector in a STEM allows efficient sampling of the transmitted electrons, and consequently increases the sensitivity of the instrument. The electrons transmitted with minor scattering are detected in the brightfield mode and provide images complementary to the darkfield mode. The transmission of electrons is proportional to the sample thickness and density thus enabling imaging of materials of different densities within a sample with high contrast at a resolution of a few nanometers (Liu 2005).

In addition to the various electron based signals, generation of SE also produces characteristic X-rays. The produced X-rays can be used for quantitative and qualitative elemental analysis of the sample. Although simultaneous use of multiple detectors in a modifiable configuration makes STEM a versatile technique, conventional STEM can however only be used to study samples that are stable in high vacuum of $10^{-5}$ to $10^{-7}$ $\mathrm{mBar}$. Amyloid fibrils are stable in high vacuum and can be studied using STEM at high 
resolution. In this thesis, STEM imaging was used to evaluate the functionalization of the aSyn fibrils with gold nanoparticles. Fibrils labelled with 1.4 and $5 \mathrm{~nm}$ diameter gold nanoparticles were visualized on ultra-thin carbon filmed copper grids in brightfield and darkfield mode (FEI SEM Verios).

\section{THESIS OVERVIEW}

Over the course of this thesis, we have strived to understand the following research questions:

1. Can the inherent polymorphism of aSyn fibrils be modulated by solution conditions?

2. What are the mechanisms that govern aSyn fibril morphology?

3. How does the morphology of the fibrils influence the Thioflavin-T fluorescence intensity?

4. Is heterologous seeding in aSyn conformation or sequence dependent?

5. Can aSyn fibrils be functionalized to produce hybrid functional structures

The knowledge generated while seeking answers to these questions is described in different chapters as listed below:

Chapter 2 assesses the role of solution conditions in reducing the routinely observed polymorphism in aSyn amyloids. Employing hi-resolution AFM we studied the morphologies of the fibrils prepared in different solution conditions. We discuss the effect of these solution conditions on fibril polymorphism.

Chapter 3 describes a longitudinal study on the morphological transitions in wt and disease associated mutants of aSyn (A30P, E46K and A53T). Using the solution conditions optimized in chapter 2, we detail the changes in the distribution of polymorphs of the selected aSyn sequences. Further, we consider the probable mechanisms that may govern the morphology of the fibrils at the studied time scale.

Chapter 4 examines the contribution of fibril morphology on the ThT fluorescence intensity. Building on the results of chapter 3, we designed experiments to study polymorph specific fibril-ThT interaction and discuss its implications for ThT based amyloid studies.

Chapter 5 evaluates the conformational compatibility between the seeds and the monomers in seeded aggregations. Based on the results of chapter 3 and 4, we 
performed cross seeded experiments to understand templating between seeds and monomers of contrasting conformations.

Chapter 6 explores the functionalization of A140C aSyn fibrils to fabricate hybrid bio-nanostructures. Using thiol-maleimide chemistry we functionalized aSyn fibrils with gold particles to make conductive nanowires.

Chapter 7 discusses the work presented in the thesis and provides an outlook for possible future directions. 


\section{Chapter 2}

\section{Solution conditions define morphological homogeneity of $\alpha$-synuclein fibrils}

Structural polymorphism is one of the inherent characteristics of amyloid proteins. Like most amyloids, human $\alpha$-synuclein ( $\alpha$ Syn), an intrinsically disordered protein, exhibits considerable heterogeneity in in vitro fibrillization reactions. The presence of polymorphic fibrils in a given sample hampers interpretation of structural studies and correlation with toxicity in most of the amyloid studies. Therefore, considerable effort has been spent on identifying aggregation conditions that result in homogeneous fibril samples. In this chapter, we examine the influence of applied solution conditions on the morphological homogeneity of fibrils of A140C and wt aSyn. We find that low salt and inclusion of EDTA in solution conditions results in a near homogeneous fibril population. The ability to produce a homogeneous fibril sample can be instrumental in understanding the detailed structural features of fibrils and the fibril assembly process. Moreover, the availability of morphologically well-defined fibrils enhances the potential for use of amyloids as biological nanomaterials.

Parts of this chapter have been published as: Arshdeep Sidhu, Ine Segers-Nolten, Vinod Subramaniam. Solution conditions define morphological homogeneity of $\alpha$-synuclein fibrils. BBA-Proteins and Proteomics, 1844 (2014) 2127-2134. 


\section{INTRODUCTION}

Self-assembled protein structures have been associated with several age related degenerative diseases like Parkinson's disease (PD), Alzheimer's disease (AD) and diabetes (Spillantini, Schmidt et al. 1997; Chiti and Dobson 2006; Stefanis 2012). Although there are defining clinical symptoms for a particular amyloid disease, they can vary considerably between patients. The heterogeneity in disease symptoms among patients has been speculated to be due to structural polymorphism or morphological heterogeneity linked to amyloids (Guo, Covell et al. 2013; Peelaerts, Bousset et al. 2015). Experimental evidence suggests that different but single dominant fibril conformations (with distinct physico-chemical properties) exist in patients with discrete disease symptoms (Lu, Qiang et al. 2013). However, in vitro aggregation studies, aimed at understanding the molecular aspects of amyloid diseases, routinely produce heterogeneous amyloid fibrils. Therefore, to improve correlation between in vitro and clinically observed biochemical characteristics of disease causing amyloid form(s) it is important to be able to study near homogeneous samples similar to those found in patients.

In addition to association with pathogenesis, the proposition that any protein sequence can assemble into amyloid form (Dobson 2003) has resulted in an extension of amyloid science from not only understanding mechanisms involved in fibrillization, diagnostics (Ono and Saji 2011; Teich, Patel et al. 2013) and potential therapeutics (Bieschke, Russ et al. 2010; Wang, Boddapati et al. 2010; Lu, Ardah et al. 2011) but also to studying their mechano-physical properties and designing functionalized fibrils via biochemical modifications for custom applications (Hamada, Yanagihara et al. 2004; Mankar, Anoop et al. 2011). In the past decade, functionalities of amyloids have been extensively explored for use as nanomaterials. Fibrillar amyloids have been shown to be promising candidates as bio-templates for tissue engineering and bio-mineralization (Chen and Rosi 2010) and as metal or polyelectrolyte conjugated nanowires (Scheibel, Parthasarathy et al. 2003; Herland, Bjork et al. 2005; Gazit 2007; Colby, Hulleman et al. 2008). However, for use as a bio-nanomaterial it is important that homogeneous fibril samples can be reproducibly formed. Thus, at present the applicability of the fibrils as bio-nanomaterials is also limited due to inherent heterogeneity in the in vitro fibrillization. 
Amyloid formation: Fibrillization is reported to be a nucleation dependent polymerization process, in which nucleation is usually induced de novo by protein monomers. However, nucleation can also be circumvented by including pre-formed fibrils (seeded aggregation) (Jarrett and Lansbury 1993; Wood, Wypych et al. 1999). The early phase of aggregation is suggested to be a complex molecular mix, where at different time points, alternative competing reaction pathways like fragmentation or surface catalyzed nucleation (secondary nucleation) may be favored (Gosal, Morten et al. 2005; Heise, Hoyer et al. 2005; Fodera, Librizzi et al. 2008; Knowles, Waudby et al. 2009; Cohen, Linse et al. 2013). The plateau phase of the aggregation reaction is an assortment of monomers, oligomers and fibrils with the morphology of the mature fibrils exhibiting appreciable heterogeneity (Heise, Hoyer et al. 2005; Kodali and Wetzel 2007; Fandrich, Meinhardt et al. 2009; Usov, Adamcik et al. 2013). Morphological heterogeneity or structural polymorphism can be inherent as seen with proteins like A $\beta$ (Goldsbury, Frey et al. 2005), aSyn (Heise, Hoyer et al. 2005; van Raaij, Segers-Nolten et al. 2006; Vilar, Chou et al. 2008), Ig light chains (Ionescu-Zanetti, Khurana et al. 1999), ovalbumin (Lara, Gourdin-Bertin et al. 2012), $\beta$-lactoglobulin and lysozyme (Lara, Adamcik et al. 2011), which form morphologically distinct assemblies under the same solution conditions. However, specific solution conditions may favor definite morphology. Numerous reports have shown the critical role of solution conditions like ionic strength, $\mathrm{pH}$, temperature, inclusion of metal ions, and other small molecules on the aggregation kinetics and morphology of the fibrils (Harper, Wong et al. 1999; Jimenez, Nettleton et al. 2002; Khurana, Ionescu-Zanetti et al. 2003; van Raaij, van Gestel et al. 2008; Xue, Hellewell et al. 2009; Adamcik, Jung et al. 2010; Bieschke, Russ et al. 2010; Pronchik, He et al. 2010; Wang, Boddapati et al. 2010; Adamcik and Mezzenga 2011; Lu, Ardah et al. 2011; Buell, Galvagnion et al. 2014; Semerdzhiev, Dekker et al. 2014). In general, factors favoring faster fibrillization like higher salt, higher temperature and low $\mathrm{pH}$ are shown to result in more heterogeneous aggregations (Campioni, Carret et al. 2014).

aSyn, like other amyloidogenic proteins assembles into fibrillar species in vitro under a range of solution conditions and displays highly polymorphic mature fibrils (Hoyer, Antony et al. 2002; Heise, Hoyer et al. 2005; Kodali and Wetzel 2007; Pronchik, He et al. 2010; Campioni, Carret et al. 2014). Furthermore, the aggregation kinetics of aSyn (wt and mutants) have been known to be highly irreproducible. However, recent 
studies have reported on new approaches to improve reproducibility of in vitro fibrillization assays (Giehm, Lorenzen et al. 2011; Buell, Galvagnion et al. 2014; Herva, Zibaee et al. 2014).

Determination of fibril morphology: Features like height, periodicity and length are typically used to describe the morphology of amyloid fibrils. Of these, height and periodicity are shown to be dependent on the number of protofilaments in the fibrils and can be determined accurately by AFM at single fibril level in a direct and modelfree manner (Harper, Wong et al. 1999; Jimenez, Nettleton et al. 2002; Khurana, Ionescu-Zanetti et al. 2003; Qin, Hu et al. 2007; Adamcik, Jung et al. 2010; Adamcik and Mezzenga 2011). Length on the other hand is critically affected by agitation during the aggregation reaction and stochastic shear forces inherent with handling, both of which favour fragmentation (Xue, Hellewell et al. 2009; Pronchik, He et al. 2010). Moreover, length analysis of the fibrils deposited on the mica surface is reported to have adsorption (during sample preparation) and detection (during analysis) bias towards shorter or longer fibrils (van Raaij, van Gestel et al. 2008; Xue, Homans et al. 2009).

In the present chapter, we study the influence of selected solution conditions on the morphological homogeneity of aSyn fibrils. We systematically imaged a large number of single fibrils at high resolution with AFM and quantified the fibril morphology with respect to their height and periodicity. The results show that the inclusion of EDTA (ethylenediaminetetraacetic acid) in the given fibrillization reaction conditions induces the formation of fibrils with uniform heights and periodicities. The impetus for the presented experiments was the desire to find an aggregation protocol which could produce near homogeneous fibril samples at the plateau phase of the ThT fluorescence assay. Availability of a homogeneous fibril sample is an important step to investigate polymorph-specific features and to explore amyloids as a bio-nanomaterial. In the following chapters, the solution conditions optimized in this chapter are used as standard aggregation conditions to study polymorph-specific biochemistry and biophysics in different contexts. 


\section{MATERIALS AND METHODS}

\section{Expression and purification of $\alpha$ Syn}

In this study, we used $\alpha$ Syn mutant protein carrying a cysteine at position 140 instead of an alanine (A140C) and wild type (wt) protein. Given the relative ease of functionalization of cysteine through the -SH group, replacement of alanine by cysteine facilitates biochemical modifications and makes this mutant an important sequence polymorph for functionalization studies. Also being at the carboxyl terminus, the residue is suggested to be exposed to the solution in the mature fibril and is less likely to interfere in the process of fibrillization (Del Mar, Greenbaum et al. 2005; Qin, Hu et al. 2007; Guilliams, El-Turk et al. 2013).

E.coli BL21 (DE3) cells transformed with the pT7-7 plasmid carrying the wt aSyn gene were cultured in 1 liter of LB medium with $100 \mu \mathrm{g} / \mathrm{ml}$ Ampicillin. At an OD of 0.60.7 , the T-7 promoter was induced by $1 \mathrm{mM}$ IPTG for 4 hours. Cells were harvested by centrifugation at $6000 \times \mathrm{g}$ for 10 minutes. The cell pellet was resuspended in $1 / 10^{\text {th }}$ of the culture volume in $10 \mathrm{mM}$ Tris- $\mathrm{HCl}$ at $\mathrm{pH}$ 8.0, $1 \mathrm{mM}$ EDTA and $1 \mathrm{mM}$ PMSF, and stirred for 1 hour at $4{ }^{\circ} \mathrm{C}$. Cells were lysed by sonication for 2 minutes. Cellular debris was removed by centrifugation at $10,000 \times \mathrm{g}$ for 20 minutes at $4{ }^{\circ} \mathrm{C}$. Nucleic acids were removed from the lysate by adding $1 \%(\mathrm{w} / \mathrm{v})$ of streptomycin sulfate and stirring for 15 minutes at $4{ }^{\circ} \mathrm{C}$, followed by centrifugation at $13,500 \times \mathrm{g}$ for 30 minutes at $4{ }^{\circ} \mathrm{C}$. aSyn was salted-out from the solution by slow addition of ammonium sulfate to a final concentration of $0.295 \mathrm{~g} / \mathrm{ml}$ with mild stirring for 1 hour at $4{ }^{\circ} \mathrm{C}$. Precipitated protein was collected by centrifugation at $13,500 \times \mathrm{g}$ for 30 minutes at $4{ }^{\circ} \mathrm{C}$. The ammonium sulfate pellet was gently resuspended in $1 / 20^{\text {th }}$ of the culture volume in $10 \mathrm{mM}$ Tris- $\mathrm{HCl}$ at pH 7.4 and filtered through a $0.22 \mu \mathrm{m}$ filter. The solution was loaded onto a $6 \mathrm{ml}$ ResourceQ column using an Åkta Purifier-system (GE Healthcare). aSyn was eluted using a linear gradient of $\mathrm{NaCl}(0-500 \mathrm{mM})$ in $10 \mathrm{mM}$ Tris- $\mathrm{HCl}$ at $\mathrm{pH} 7.4$ at a flow-rate of $3 \mathrm{ml} / \mathrm{min}$ over 20 column volumes in $1 \mathrm{ml}$ fractions. Fractions were checked for $\alpha \mathrm{Syn}$ using SDS-PAGE and pooled. The pooled sample was concentrated (Vivaspin-20, $10,000 \mathrm{kDa}$ ) to a volume of $<2.5 \mathrm{ml}$. The sample was desalted with a PD-10 column (GE Healthcare) using $10 \mathrm{mM}$ Tris- $\mathrm{HCl}$ at $\mathrm{pH}$ 7.4. The volume was adjusted with Tris- $\mathrm{HCl}$, at $\mathrm{pH} 7.4$ to a concentration of $250 \mu \mathrm{M}$, and divided into aliquots of $0.5 \mathrm{ml}$ and stored at $-80{ }^{\circ} \mathrm{C}$. The A140C mutant construct was generated by site directed mutagenesis 
using a QuikChange II Site-Directed Mutagenesis Kit (Agilent Technologies). A140C protein was expressed and purified according to the same protocol as wt aSyn, with additional inclusion of $1 \mathrm{mM}$ DTT in all buffers.

\section{Fibrillization reaction}

$250 \mu \mathrm{M}$ monomeric stocks of wt and A140C mutant $\alpha$ Syn frozen at $-80^{\circ} \mathrm{C}$ were thawed and filtered through $0.02 \mu \mathrm{m}, 10 \mathrm{~mm}$ Anotop 10 Whatman syringe filters. Aggregation reactions were set up with $100 \mu \mathrm{M}$ A140C $\alpha$ Syn, $10 \mathrm{mM}$ Tris-HCl, 1 or $10 \mathrm{mM} \mathrm{NaCl}$, (+/-) $0.1 \mathrm{mM}$ EDTA, $2 \mathrm{mM}$ fresh DTT (dithiothreitol) at pH 7.4 and with $100 \mu \mathrm{M}$ wt aSyn, $10 \mathrm{mM}$ Tris- $\mathrm{HCl}, 1$ or $10 \mathrm{mM} \mathrm{NaCl}$, (+/-) $0.1 \mathrm{mM}$ EDTA at $\mathrm{pH}$ 7.4. All reactions were prepared in triplicate with volumes of $400 \mu \mathrm{l}$ each in $2 \mathrm{ml}$ Lo-Bind round bottom Eppendorf centrifuge tubes and were incubated at $37^{\circ} \mathrm{C}$ with $500 \mathrm{rpm}$ orbital shaking in an Eppendorf Thermo-mixer comfort. Fibrillization using Tris (2-carboxyethyl) phosphine hydrochloride (TCEP-Fluka BioChemika) was done in a similar manner as with DTT with minor differences. The protein aliquots from $-80^{\circ} \mathrm{C}$ were desalted using a $5 \mathrm{ml}$ Zeba spin desalting column (7k MWCO, Pierce Biotechnology) to remove any residual DTT. A $5 \mathrm{mM}$ TCEP solution (stock concentration) was prepared in $10 \mathrm{mM}$ Tris- $\mathrm{HCl}, 10 \mathrm{mM} \mathrm{NaCl}$ at $\mathrm{pH}$ 7.4. Since TCEP is acidic, the $\mathrm{pH}$ was re-adjusted to 7.4 using $\mathrm{NaOH}$. $100 \mu \mathrm{M}$ A140C $\alpha$ Syn solutions were prepared in $10 \mathrm{mM}$ Tris- $\mathrm{HCl}, 10 \mathrm{mM}$ $\mathrm{NaCl}, 2 \mathrm{mM}$ TCEP (final concentrations) at $\mathrm{pH}$ 7.4.

\section{Thioflavin-T assay}

Progress of fibrillization was followed by a Thioflavin-T (ThT) fluorescence assay. A stock solution of $1 \mathrm{mM}$ ThT was prepared in $50 \mathrm{mM}$ glycine- $\mathrm{NaOH}$ buffer, $\mathrm{pH} 8.2$ and filtered through a $0.22 \mu \mathrm{m}$ syringe filter. At each time point (every 24 hours), $5 \mu \mathrm{l}$ aliquots of sample were drawn and diluted in $2 \mathrm{ml}$ of $5 \mu \mathrm{M}$ ThT working solution diluted in glycine- $\mathrm{NaOH}$ buffer, $\mathrm{pH}$ 8.2. Fluorescence intensity was measured in triplicate on a Cary Eclipse fluorescence spectrophotometer (Varian), with excitation at $457 \mathrm{~nm}$ and emission detection from 475 to $600 \mathrm{~nm}$ using slit widths of $10 \mathrm{~nm}$. ThT curves were prepared by plotting the emission intensity readings at $485 \mathrm{~nm}$ versus aggregation time; triplicate values were averaged and blank subtracted. 


\section{Fluorescence microscopy}

For fluorescence microscopy, aggregation reactions were diluted 50 times in aggregation buffer (final concentration $2 \mu \mathrm{M}$ aSyn monomer equivalent) and ThT was added to a final concentration of $10 \mu \mathrm{M} .5 \mu \mathrm{l}$ of the diluted aggregation reactions were placed on a clean microscope slide and covered by a clean cover slip. The edges of the samples were sealed with lacquer and the samples were allowed to settle for 24 hours. Fluorescence images were acquired in widefield mode using an oil immersion objective with $100 \times$ magnification $(\mathrm{NA}=1.25)$ from Nikon on a Nikon Eclipse Ti microscope equipped with a mercury arc lamp (Intensilight, Nikon) for excitation. For imaging of ThT fluorescence, a filter cube composed of a 445/465 $\mathrm{nm}$ band pass excitation filter, a $458 \mathrm{~nm}$ long pass dichroic beam splitter and a 470/500 $\mathrm{nm}$ band pass emission filter was used.

\section{Residual monomer concentration (RMC)}

The amount of monomers left in the aggregation reaction after attaining the plateau phase in a ThT assay was determined by centrifugation of $200 \mu \mathrm{l}$ aliquots of aggregation reactions at $21,000 \times \mathrm{g}$ for 1 hour in an IEC Micromax microcentrifuge (Thermo Fisher). The supernatant recovered from the centrifuged samples was filtered through $0.02 \mu \mathrm{m}, 10 \mathrm{~mm}$ Anotop10 Whatman syringe filters and the absorbances were measured at 280 and $330 \mathrm{~nm}$ in a Shimadzu UV-2401 PC spectrophotometer. The absorption values at $280 \mathrm{~nm}$ were corrected for scattering contributions $\left(\mathrm{A}_{330}\right)$ (Grimsley 2004) before calculating the residual monomer concentration, using an extinction coefficient of 5600 and $5745 \mathrm{M}^{-1} \cdot \mathrm{cm}^{-1}$ for wt and A140C aSyn, respectively.

\section{Atomic force microscopy (AFM)}

AFM samples were prepared by adsorbing $20 \mu \mathrm{l}$ of fibril sample, 5 to 10 times diluted in $10 \mathrm{mM}$ Tris- $\mathrm{HCl}, 10 \mathrm{mM} \mathrm{NaCl}$ at $\mathrm{pH} 7.4$ on freshly cleaved mica (Muscovite mica, V-1 quality, EMS) for 4 minutes, followed by 2 gentle washes with $100 \mu$ of fresh Milli$\mathrm{Q}$ water and drying in a gentle stream of nitrogen gas. AFM images were acquired on a Bioscope Catalyst (Bruker) in soft tapping mode in air using a silicon probe, NSC36 tip $\mathrm{B}$ (tip radius $<50 \mathrm{~nm}$ ) with force constant of $1.75 \mathrm{~N} / \mathrm{m}$ (MikroMasch). All images were captured with a resolution of $512 \times 512$ pixels per image with a scan rate of $0.5 \mathrm{~Hz}$ and a scan size of $1.7 \times 1.7 \mu \mathrm{m}$. For each solution condition about 100 non-overlapping 
fibrils more than $1 \mu \mathrm{m}$ in length were qualitatively analyzed by Scanning Probe Image Processor-6.02 (Image Metrology) software; for quantitative analysis of height $(h)$ and periodicity $(p)$ a custom written Matlab script using DIPimage toolbox (version 2.3) was used (Faas, Rieger et al. 2009). The script is based on quantitative analysis of AFM images as described elsewhere (van Raaij, Segers-Nolten et al. 2006).

\section{DTNB assay}

DTNB [5, 5'-dithiobis-(2-nitrobenzoic acid)] assay was carried out to quantify the amount of active DTT (free thiols) in aggregation reactions with and without EDTA. 10 mM DTNB stock solution was prepared in $0.1 \mathrm{M}$ sodium phosphate buffer, $\mathrm{pH} 8.0$ and filtered through a $0.22 \mu \mathrm{m}$ syringe filter. For each time point reading, a standard curve for DTT over a concentration range of 5 to $50 \mu \mathrm{M}$ was prepared with a working concentration of $1 \mathrm{mM}$ DTNB. $5 \mu$ aggregation aliquots were drawn at each time point and added to $1 \mathrm{ml}$ of $1 \mathrm{mM}$ DTNB solution in $0.1 \mathrm{mM}$ sodium phosphate buffer $\mathrm{pH} 8.0$ and absorbance was measured at $412 \mathrm{~nm}$ on a Shimadzu UV-2401 PC spectrophotometer. The concentration of the free thiols in aggregation reactions was calculated using $\mathrm{A}_{412 \mathrm{~nm}}$ absorbance readings and the slope of the standard curve.

\section{Native polyacrylamide gel electrophoresis}

Clear native polyacrylamide gel electrophoresis (nPAGE) was performed to probe the conformers/oligomeric assemblies formed during the aggregation reaction. $12 \%$ resolving nPAGE was cast with $4 \%$ stacking gel and electrophoresed in $300 \mathrm{mM}$ of BisTris at $\mathrm{pH} 7.4$ as the anode buffer and $300 \mathrm{mM}$ of Bis-Tris, $500 \mathrm{mM}$ Tricine at $\mathrm{pH} 7.4$ as the cathode buffer. A constant current of $4 \mathrm{~mA}$ was applied while running in the stacking gel and of $6 \mathrm{~mA}$ in the resolving gel. The sample for electrophoresis was prepared in a $2 \times$ sample loading buffer $(300 \mathrm{mM}$ of Bis-Tris $\mathrm{pH} 7.4,3 \mathrm{M}$ aminocaproic acid, $0.001 \%$ Ponceau S). $10 \mu$ protein samples were drawn fresh from the aggregation reaction and electrophoresed. Electrophoresed gels were stained in Coomassie Brilliant Blue R250 staining solution in acetic acid: methanol: water (1:4:5) for 20 minutes and destained in acetic acid: methanol: water (1:4:5). 


\section{RESULTS AND DISCUSSION}

\section{Thioflavin-T fluorescence assay}

A number of in vitro studies on amyloidogenic proteins have shown the crucial role of salt concentration on aggregation kinetics. In general, higher salt concentrations lead to faster self-assembly (Hoyer, Antony et al. 2002; Fujiwara, Matsumoto et al. 2003; Hoyer, Cherny et al. 2004; Marek, Patsalo et al. 2012; Buell, Hung et al. 2013). This enhanced aggregation rate is attributed to sufficient charge screening at higher salt concentrations. Thus reducing the repulsive forces among the monomers and facilitating intra- and intermolecular interactions governed by hydrophobicity (Raman, Chatani et al. 2005; Adamcik and Mezzenga 2011). Intramolecular hydrophobic interactions induce partially folded conformations in intrinsically disordered proteins like aSyn, one or more of which could be a nucleation promoting conformation (Uversky, Li et al. 2001). The possibility of attaining conformations with subtle structural differences in a given solution condition decisively influences alternative fibril assembly pathways during nucleation, and is proposed to be the basis of heterogeneity (Goldsbury, Frey et al. 2005; Petkova, Leapman et al. 2005; Bousset, Pieri et al. 2013). A recent study on strain like behavior of aSyn amyloids shows formation of fibril (non-periodic) or ribbon (periodic) type of assemblies, highlighting differences in monomer conformations induced by specific aggregation conditions (Bousset, Pieri et al. 2013; Gath, Bousset et al. 2014).

In addition to influencing the aggregation kinetics, high salt concentrations are also believed to lead to clustering of the fibrils. To determine the optimal salt concentration that does not induce clustering we prepared fibrils (A140C and wt) at $1 \mathrm{mM} \mathrm{NaCl}$ and added additional salt up to a concentration of $200 \mathrm{mM}$. Imaging of the fibrils in the presence of ThT by fluorescence microscopy showed that the fibrils (A140C and wt aSyn), which were disperse at $1 \mathrm{mM} \mathrm{NaCl}$, begin to cluster at about $50 \mathrm{mM} \mathrm{NaCl}$ concentration (Figure 2.1). Such supramolecular aggregates are not amenable to single fibril level morphological studies by AFM. Therefore, with an aim to reduce heterogeneity and to avoid fibril clustering we chose to fibrillize aSyn at low salt concentrations of 1 and $10 \mathrm{mM} \mathrm{NaCl}$, instead of more frequently used concentrations of $50 \mathrm{mM}$ or $100 \mathrm{mM} \mathrm{NaCl}$. The fibril clustering observed in our study is in agreement with recent reports probing the effect of solution conditions on aSyn fibrillization. In 
these studies, the authors demonstrate the tendency of fibrils to form higher order assemblies and floccules at higher $\mathrm{NaCl}$ concentrations (Buell, Galvagnion et al. 2014; Semerdzhiev, Dekker et al. 2014).

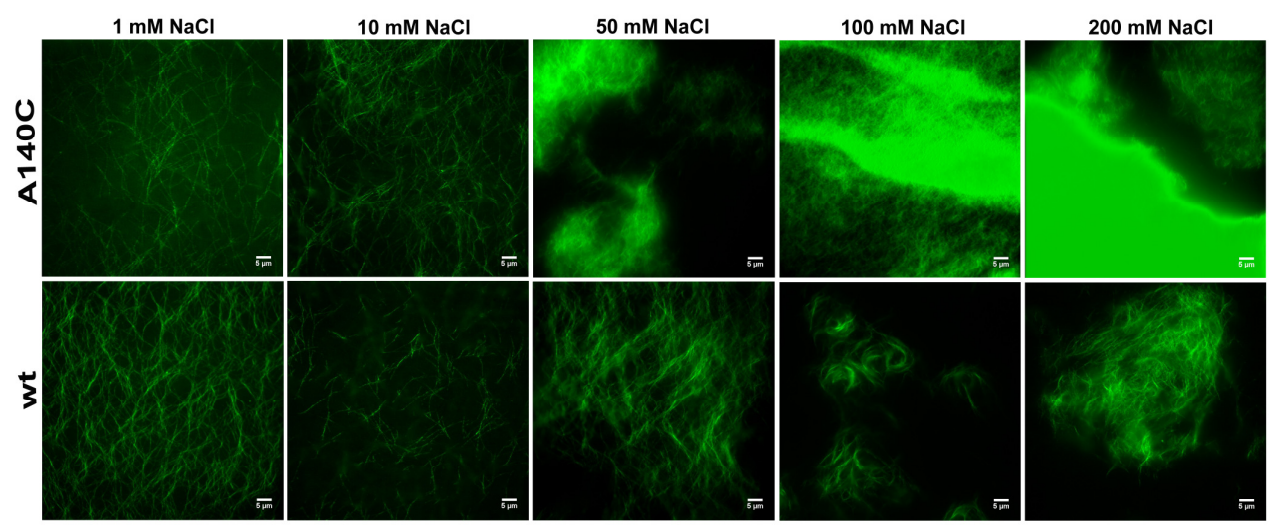

Figure 2.1 Widefield fluorescence microscopy images of A140C and wt aSyn fibrils after binding of ThT, showing clustering of fibrils with increasing salt concentration. Scale bar: $5 \mu \mathrm{m}$.

A140C aSyn was aggregated in $10 \mathrm{mM}$ Tris- $\mathrm{HCl}, 1$ or $10 \mathrm{mM} \mathrm{NaCl},(+/-) 0.1 \mathrm{mM}$ EDTA, $2 \mathrm{mM}$ fresh DTT at pH 7.4. $2 \mathrm{mM}$ DTT was included to prevent intermolecular disulfide bond formation by the introduced cysteine residue. Inclusion of 0.1 to $1 \mathrm{mM}$ of EDTA has been shown to enhance the stability of thiol additives in protein purification against oxidation by trace metal ions (Stevens 1983). A DTNB assay to determine the optimal EDTA concentration to maximize half-life of DTT showed 0.1 mM EDTA to be as effective as higher concentrations (data not shown). Thus, $0.1 \mathrm{mM}$ EDTA was chosen as the concentration in EDTA supplemented aggregation reactions.

We observed two clear trends in the ThT curves following fibrillization of A140C mutant in the above-mentioned solution conditions. Reactions in the presence of 10 $\mathrm{mM} \mathrm{NaCl}$ showed higher fluorescence intensities compared to those at $1 \mathrm{mM} \mathrm{NaCl}$ salt concentration. In addition, reactions in the presence of $0.1 \mathrm{mM}$ EDTA reproducibly showed higher fluorescence intensities in the plateau phase of the aggregation reaction as compared to reactions without EDTA (Figure 2.2A). The measured fluorescence intensities of the reactions with $1 \mathrm{mM} \mathrm{NaCl}$ in the presence of EDTA were comparable to those of the reactions with only $10 \mathrm{mM} \mathrm{NaCl}$. In a control experiment, A140C fibrils prepared in $10 \mathrm{mM}$ Tris- $\mathrm{HCl}, 10 \mathrm{mM} \mathrm{NaCl}$ and $2 \mathrm{mM}$ DTT at $\mathrm{pH}$ 7.4, were titrated 
with $0.08 \mathrm{mM}$ to $2.5 \mathrm{mM}$ EDTA at $\mathrm{pH} 7.4$ to examine the effect, if any, of EDTA on the ThT assay. The results showed no noticeable difference in ThT intensities in the presence/absence of EDTA (data not shown).

An analogous set of experiments with $100 \mu \mathrm{M}$ wt aSyn in $10 \mathrm{mM}$ Tris- $\mathrm{HCl}$, with 1 or $10 \mathrm{mM} \mathrm{NaCl}$ in the absence and presence of $0.1 \mathrm{mM}$ EDTA at $\mathrm{pH} 7.4$ was done to ascertain if the trend observed in A140C is also seen with wt aSyn. Fibrillization reactions in the presence of $0.1 \mathrm{mM}$ EDTA reproducibly showed higher ThT fluorescence values as compared to reactions without EDTA (Figure 2.2B). Varying the concentration of EDTA from $0.05,0.1$ to $0.2 \mathrm{mM}$ for further optimization of wt aggregations did not show any significant difference in the final fluorescence values (Figure 2.3A).
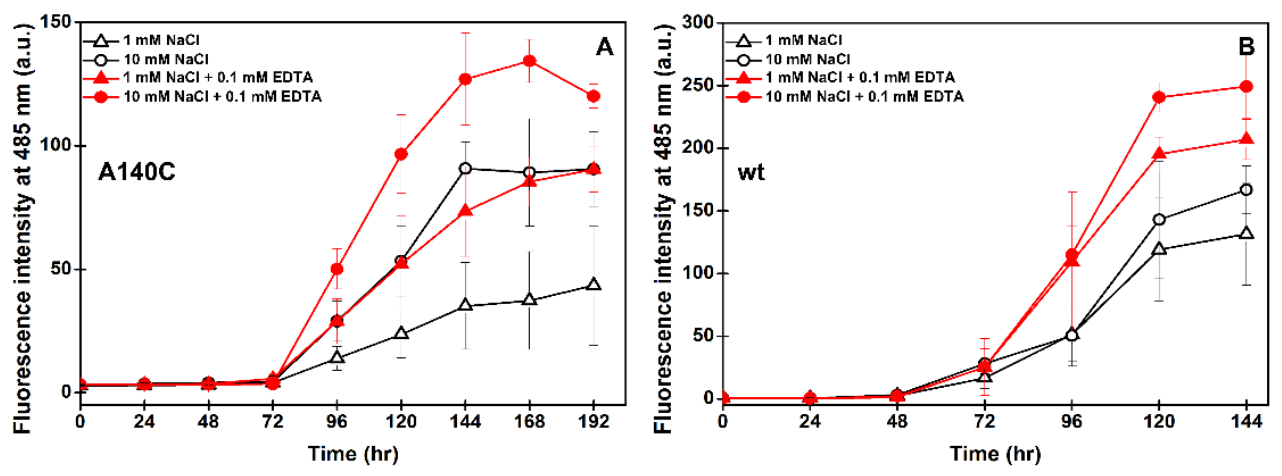

Figure 2.2 Kinetics of (A) A140C and (B) wt aSyn aggregation measured by ThT fluorescence as a function of $\mathrm{NaCl}$ concentration in the absence or presence of $0.1 \mathrm{mM}$ EDTA. Aggregation buffer for A140C included $10 \mathrm{mM}$ Tris-HCl, $2 \mathrm{mM}$ DTT at $\mathrm{pH} 7.4,1$ or $10 \mathrm{mM} \mathrm{NaCl}$; with or without $0.1 \mathrm{mM}$ EDTA and for wt aSyn aggregation, $10 \mathrm{mM}$ Tris- $\mathrm{HCl}$ at $\mathrm{pH} 7.4,1$ or $10 \mathrm{mM} \mathrm{NaCl}$, with or without 0.1 mM EDTA. Error bars denote standard deviation among triplicates.

aSyn aggregation is reported to be a first order nucleation dependent polymerization reaction (Wood, Wypych et al. 1999). In fibrillization with monomers, primary nucleation is dominant and is characterized by a sigmoidal growth curve in the ThT assay (Cohen, Vendruscolo et al. 2012). Higher ThT intensities in the presence of EDTA could thus indicate more cross- $\beta$ structure. Determination of residual monomer concentration (RMC) at the plateau phase of ThT curves showed that reactions with EDTA had less monomers left for A140C and wt aggregations (Figure 2.3B). Together, data from ThT assays and RMC analyses suggest that a larger fraction of monomers is 
incorporated in ThT positive fibrils in the presence of EDTA. Lower final fluorescence intensities and higher RMC found for $1 \mathrm{mM} \mathrm{NaCl}$ reactions on the other hand suggest lower cross- $\beta$ content and less uptake of monomers in fibrils. However, it is also possible that ThT may exhibit differential binding to fibrils prepared under different solution conditions. Calculation of per $\mu \mathrm{M}$ contribution of fluorescence from each fibril sample using RMC and final fluorescence values shows that fibrils prepared in the presence of EDTA have 10 to $40 \%$ higher fluorescence per $\mu \mathrm{M}$ of protein incorporated into fibrils (Table 2.1). Thus, in solution conditions including EDTA not only more monomers are included into the fibrils but also the fibrils formed likely bind ThT in a more efficient manner.
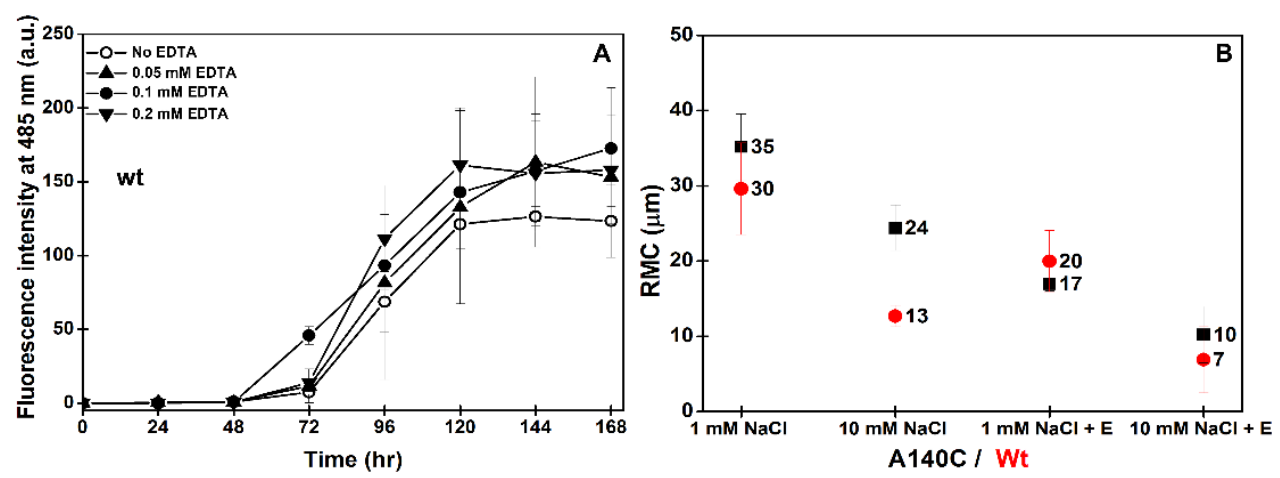

Figure 2.3 (A) Kinetics of wt aSyn aggregation measured by ThT fluorescence as a function of EDTA concentration. Aggregation buffer included $10 \mathrm{mM}$ Tris- $\mathrm{HCl}, 10 \mathrm{mM} \mathrm{NaCl}$ at $\mathrm{pH} 7.4$ with either no EDTA, 0.05 mM EDTA, $0.1 \mathrm{mM}$ EDTA or $0.2 \mathrm{mM}$ EDTA. (B) RMC determined from aggregation reactions of A140C and wt aSyn at the plateau phase of ThT curves. Error bars denote standard deviation among triplicates.

\section{AFM imaging and morphology analysis}

Fibrils from the plateau phase of fibrillization reactions (after 192-216 hours), were imaged by AFM using soft tapping mode in air. The fibrils (A140C and wt) were typically several micrometers long, and exhibited distinctive twisted morphology (Figure 2.4) (van Raaij, Segers-Nolten et al. 2006). The key morphological features of twisted amyloid fibrils are their height and periodicity, characteristics that can be experimentally measured with high accuracy using AFM (Khurana, Ionescu-Zanetti et al. 2003; Adamcik, Jung et al. 2010). For each solution condition, around 100 non- 
overlapping fibrils of at least $1 \mu \mathrm{m}$ length were quantitatively analyzed for height and periodicity.

Table 2.1 Estimation of relative fluorescence per $\mu \mathrm{M}$ of fibrils at the plateau phase of ThT curves prepared in the presence and absence of EDTA. The calculated data are based on the RMC values indicated in Figure 2.3B and the final fluorescence values at 192 hours for A140C (Figure 2.2A) and at 144 hours for wt (Figure 2.2B).

\begin{tabular}{|c|c|c|c|c|c|c|}
\hline & & F.F value & $\begin{array}{l}\text { RMC } \\
(\mu \mathrm{M})\end{array}$ & $\begin{array}{l}\text { aSyn } \\
\text { fibrils }\end{array}$ & $\begin{array}{l}\text { orming } \\
\text { - RMC) }\end{array}$ & $\begin{array}{c}\text { Fluorescence per } \mu \mathrm{M} \\
\text { of fibrillar } \alpha \mathrm{Syn}\end{array}$ \\
\hline \multirow[t]{4}{*}{ A140C } & $1 \mathrm{mM} \mathrm{NaCl}$ & 43 & 35 & & & 0.66 \\
\hline & $10 \mathrm{mM} \mathrm{NaCl}$ & 90 & 24 & & & 1.18 \\
\hline & $\begin{array}{l}1 \mathrm{mM} \mathrm{NaCl}+ \\
0.1 \mathrm{mM} \mathrm{EDTA}\end{array}$ & 90 & 17 & & & 1.08 \\
\hline & $\begin{array}{l}10 \mathrm{mM} \mathrm{NaCl}+ \\
0.1 \mathrm{mM} \mathrm{EDTA}\end{array}$ & 120 & 10 & & & 1.33 \\
\hline \multirow[t]{5}{*}{$w t$} & $1 \mathrm{mM} \mathrm{NaCl}$ & 131 & 30 & & & 1.87 \\
\hline & $10 \mathrm{mM} \mathrm{NaCl}$ & 166 & 13 & & & 1.91 \\
\hline & $\begin{array}{l}1 \mathrm{mM} \mathrm{NaCl}+ \\
0.1 \mathrm{mM} \mathrm{EDTA}\end{array}$ & 207 & 20 & & & 2.59 \\
\hline & $\begin{array}{l}10 \mathrm{mM} \mathrm{NaCl}+ \\
0.1 \mathrm{mM} \text { EDTA }\end{array}$ & 250 & 7 & & & 2.69 \\
\hline & \multicolumn{5}{|c|}{ Ratio (fluorescence per $\mu \mathrm{M}$ of fibrils) } & $\begin{array}{l}\text { \% higher fluorescence } \\
\text { in + EDTA reactions }\end{array}$ \\
\hline \multirow[t]{2}{*}{$A 140 C$} & \multicolumn{2}{|c|}{$1 \mathrm{mM} \mathrm{NaCl}-/+$ EDTA } & & $0.66 / 1.08$ & 61.0 & 39.0 \\
\hline & \multicolumn{2}{|c|}{$10 \mathrm{mM} \mathrm{NaCl}-/+$ EDTA } & & $1.18 / 1.33$ & 88.8 & 11.2 \\
\hline \multirow[t]{2}{*}{$w t$} & \multicolumn{2}{|c|}{$1 \mathrm{mM} \mathrm{NaCl}-/+$ EDTA } & & $1.87 / 2.59$ & 72.2 & 27.8 \\
\hline & \multicolumn{2}{|c|}{$10 \mathrm{mM} \mathrm{NaCl}-/+$ EDTA } & & $1.91 / 2.69$ & 71.0 & 29.0 \\
\hline
\end{tabular}

F.F value: final fluorescence value

The periodicities of A140C fibrils grown in $10 \mathrm{mM} \mathrm{NaCl}$ as well as $1 \mathrm{mM} \mathrm{NaCl}$ showed a broad distribution, with average periodicities of $99 \pm 32 \mathrm{~nm}$ and $109 \pm 20 \mathrm{~nm}$, respectively. Interestingly, the fibril population produced in $10 \mathrm{mM} \mathrm{NaCl}$ appeared to have three distinct groups with average periodicities around 50, 100 and $150 \mathrm{~nm}$. However, fibrils grown in the same salt conditions, but in the presence of $0.1 \mathrm{mM}$ EDTA showed a significantly narrower distribution of periodicities with an average value of $102 \pm 6 \mathrm{~nm}$ for $10 \mathrm{mM} \mathrm{NaCl}$ and $105 \pm 6 \mathrm{~nm}$ for $1 \mathrm{mM} \mathrm{NaCl}$ salt concentrations (Figures 2.5 and 2.6 and Table 2.2). Thus, the homogeneity in periodicity of the A140C fibrils appears to be sensitive to the presence of EDTA. In other amyloid forming proteins like 


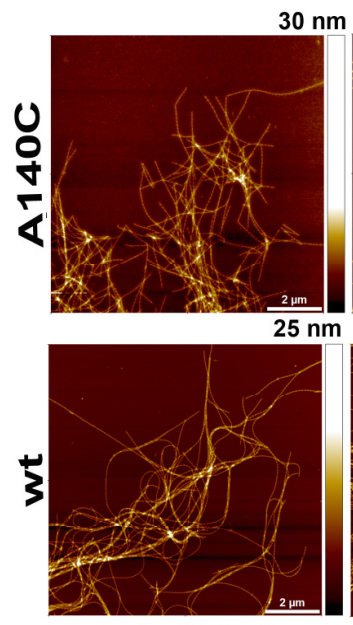

$1 \mathrm{mM} \mathrm{NaCl}$
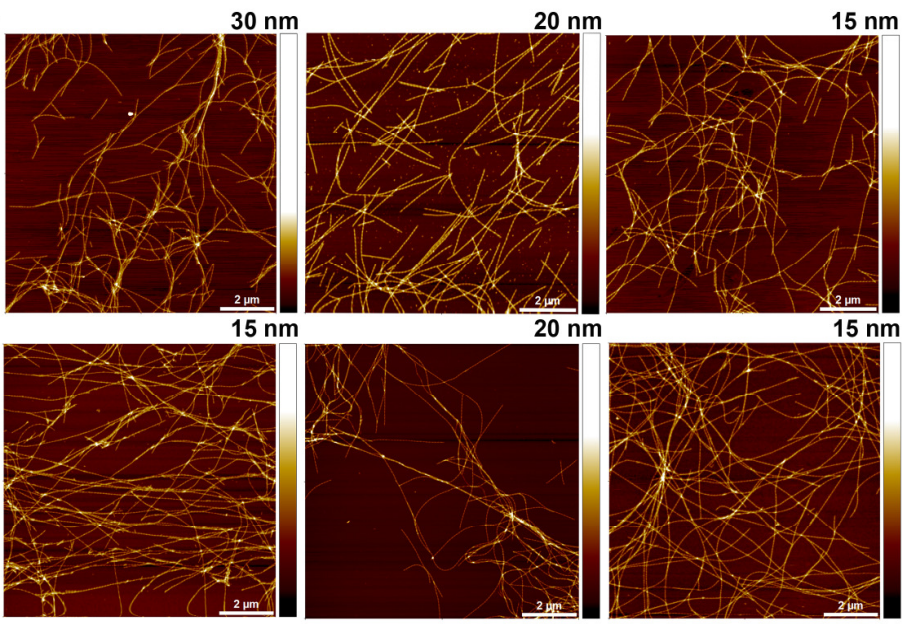

$10 \mathrm{mM} \mathrm{NaCl}$

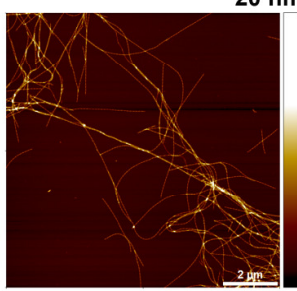

$1 \mathrm{mM} \mathrm{NaCl}+$ EDTA

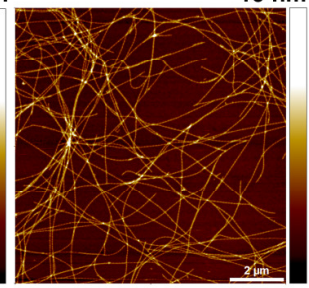

$10 \mathrm{mM} \mathrm{NaCl}+$ EDTA

Figure 2.4 Overview AFM height images of A140C and wt aSyn fibrils from the plateau phase of ThT assays. Aggregation conditions included $10 \mathrm{mM}$ Tris-HCl, $2 \mathrm{mM}$ DTT (only for A140C) at pH 7.4 with $1 \mathrm{mM}$ or $10 \mathrm{mM} \mathrm{NaCl}$ and with or without $0.1 \mathrm{mM}$ EDTA. Image size: $10 \times 10 \mu \mathrm{m}$. Note: Different dilutions were used to prepare AFM samples from aggregation reactions with different solution conditions. Thus, the amount of fibrils formed under the given conditions cannot be estimated from these representative images.

$\beta$-lactoglobulin (Adamcik and Mezzenga 2011) and insulin (Jimenez, Nettleton et al. 2002) fibril periodicity is reported to vary as a function of fibril height, which is explained by the Hierarchical Assembly Model (HAM), where successive association of protofilaments into protofibrils and fibrils accompanied by mutual twisting gives rise to various periodicities that correlate with measured heights (Harper, Wong et al. 1999; Jimenez, Nettleton et al. 2002; Khurana, Ionescu-Zanetti et al. 2003; Qin, Hu et al. 2007; Adamcik and Mezzenga 2011). The measured fibril heights in our study however, were not considerably different for fibrils prepared in 1 or $10 \mathrm{mM} \mathrm{NaCl}$ and with or without EDTA. The average heights for fibrils were $6.5 \pm 0.7(1 \mathrm{mM} \mathrm{NaCl}), 6.3 \pm 0.4(10 \mathrm{mM}$ $\mathrm{NaCl}), 6.3 \pm 0.6(1 \mathrm{mM} \mathrm{NaCl}$ with EDTA $)$ and $6.4 \pm 0.4(10 \mathrm{mM} \mathrm{NaCl}$ with EDTA $)$. In addition, it is noteworthy that fibrils formed in $10 \mathrm{mM} \mathrm{NaCl}$ without EDTA and displaying distinct periodicities of 50,100 and $150 \mathrm{~nm}$ did not show differences in height expected for hierarchical assembly (Figure 2.6C and Table 2.2). This observation suggests that the morphological parameters of height and periodicity in mature aSyn fibrils formed under the given solution conditions are not coupled. 
Table 2.2 Summary of average height and periodicity measurement of A140C and wt aSyn fibrils ( \pm stdv). Number of fibrils analyzed for each solution condition is shown in parentheses.

\begin{tabular}{|c|c|c|c|c|c|}
\hline \multirow[t]{2}{*}{$0.1 \mathrm{mM}$ EDTA } & \multicolumn{2}{|c|}{ A140C } & \multicolumn{2}{|c|}{ wt } & \multirow{2}{*}{$\begin{array}{c}\text { Morphological } \\
\text { observables }\end{array}$} \\
\hline & $1 \mathrm{mM} \mathrm{NaCl}$ & $10 \mathrm{mM} \mathrm{NaCl}$ & $1 \mathrm{mM} \mathrm{NaCl}$ & $10 \mathrm{mM} \mathrm{NaCl}$ & \\
\hline- & $\begin{array}{c}6.5 \pm 0.7 \\
(100)\end{array}$ & $\begin{array}{c}6.3 \pm 0.4 \\
(106)\end{array}$ & $\begin{array}{c}6.7 \pm 0.4 \\
(104)\end{array}$ & $\begin{array}{c}6.1 \pm 0.7 \\
(100)\end{array}$ & $\begin{array}{l}\text { Height }(h) \\
\quad(\mathrm{nm})\end{array}$ \\
\hline+ & $\begin{array}{c}6.3 \pm 0.6 \\
(78)\end{array}$ & $\begin{array}{l}6.4 \pm 0.4 \\
(100)\end{array}$ & $\begin{array}{c}6.7 \pm 0.5 \\
(100)\end{array}$ & $\begin{array}{c}6.9 \pm 0.6 \\
(105)\end{array}$ & \\
\hline- & $\begin{array}{c}109 \pm 20 \\
(100)\end{array}$ & $\begin{array}{c}99 \pm 32 \\
(106)\end{array}$ & $\begin{array}{c}110 \pm 10 \\
(104)\end{array}$ & $\begin{array}{c}119 \pm 24 \\
(100)\end{array}$ & $\begin{array}{c}\text { Periodicity }(p) \\
(\mathrm{nm})\end{array}$ \\
\hline+ & $\begin{array}{c}105 \pm 6 \\
(78)\end{array}$ & $\begin{array}{c}102 \pm 6 \\
(100)\end{array}$ & $\begin{array}{c}108 \pm 7 \\
(100)\end{array}$ & $\begin{array}{c}107 \pm 9 \\
(105)\end{array}$ & \\
\hline
\end{tabular}
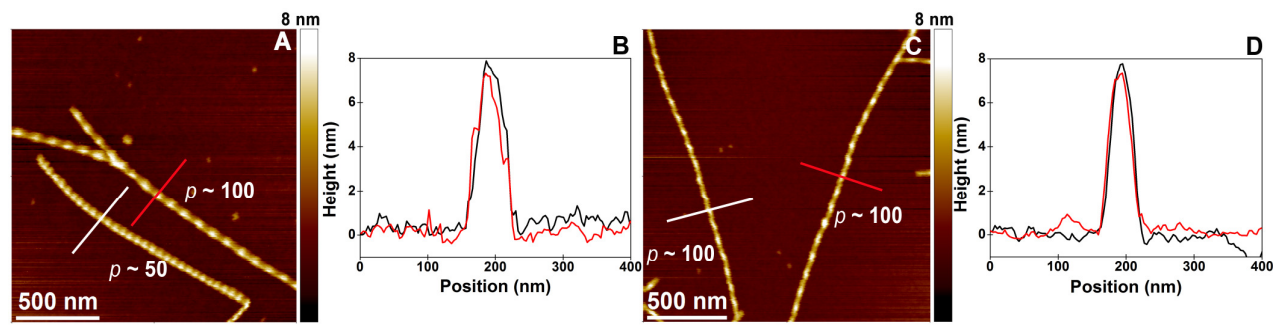

Figure 2.5 Representative AFM height images of A140C aSyn (A, C) and corresponding height profiles (B, D). Tapping mode AFM height images of A140C aSyn fibrils prepared in $10 \mathrm{mM}$ Tris$\mathrm{HCl}, 10 \mathrm{mM} \mathrm{NaCl}, 2 \mathrm{mM}$ DTT at $\mathrm{pH} 7.4$ and in the absence (A) or presence (C) of $0.1 \mathrm{mM}$ EDTA. White and red lines in (A) and (C) indicate positions of line profiles depicted in (B) and (D). Labels $(50,100)$ signify approximate value for periodicity $(p)$ of the fibrils in $n$ m. AFM image size: $1.7 \times 1.7$ $\mu \mathrm{m}$. 


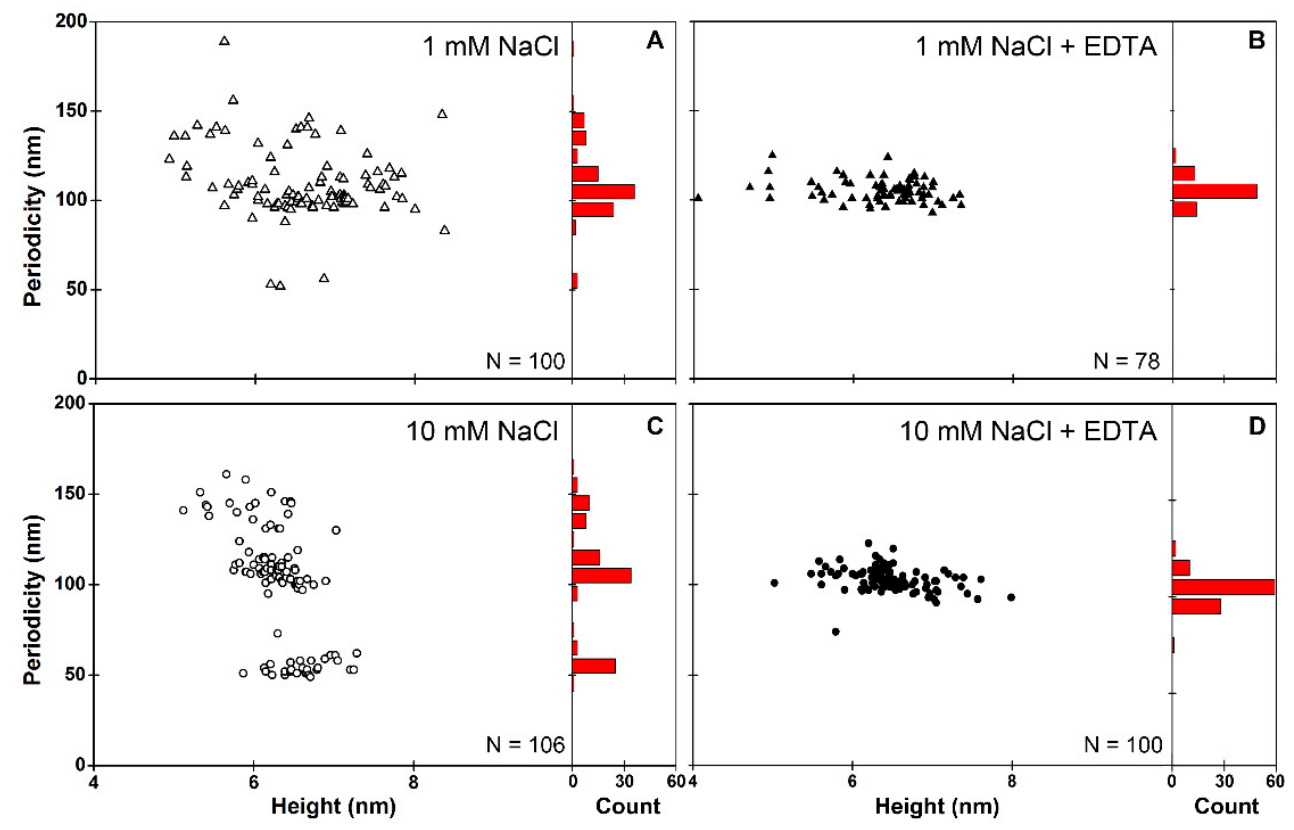

Figure 2.6 Scatter plots representing quantitative morphological features of A140C aSyn fibrils prepared in $10 \mathrm{mM}$ Tris- $\mathrm{HCl}, 2 \mathrm{mM}$ DTT at $\mathrm{pH} 7.4$ with $1 \mathrm{mM} \mathrm{NaCl}$ or $10 \mathrm{mM} \mathrm{NaCl}$ in the absence $(A, C)$ or presence $(B, D)$ of $0.1 \mathrm{mM}$ EDTA. Next to each scatter plot a histogram in red shows the distribution of periodicities for the fibrils. Bin size: $10 \mathrm{~nm}$. (A, C) Fibrils grown in the absence of EDTA display a broad distribution of periodicities; notably aggregations in $10 \mathrm{mM} \mathrm{NaCl}$ result in three fibril groups with average periodicities around 50,100 and $150 \mathrm{~nm}$. (B, D) Fibrils produced in the presence of EDTA show one dominant population corresponding to an average periodicity of about $100 \mathrm{~nm}$. Fibril periodicities show no apparent correlation with heights in the absence $(A, C)$ or presence $(B, D)$ of EDTA.

Next, a parallel set of experiments probing the effect of inclusion of EDTA on height and periodicity of wt aSyn fibrils was done to assess if the homogeneity in periodicity with EDTA is limited to the A140C mutant or is seen with wt aSyn aggregation as well. Fibrillization was performed using $100 \mu \mathrm{M}$ wt $\alpha$ Syn in 1 or $10 \mathrm{mM} \mathrm{NaCl}$, with and without $0.1 \mathrm{mM}$ EDTA at $\mathrm{pH}$ 7.4. The fibrils formed at $1 \mathrm{mM} \mathrm{NaCl}$ showed homogeneous periodicity of $110 \pm 10 \mathrm{~nm}$ (no EDTA) and $108 \pm 7 \mathrm{~nm}(0.1 \mathrm{mM}$ EDTA). Akin to A140C, at $10 \mathrm{mM} \mathrm{NaCl}$ concentration wt aSyn fibril periodicity was more defined in the presence of EDTA with an average value of $107 \pm 9 \mathrm{~nm}$ as compared to 


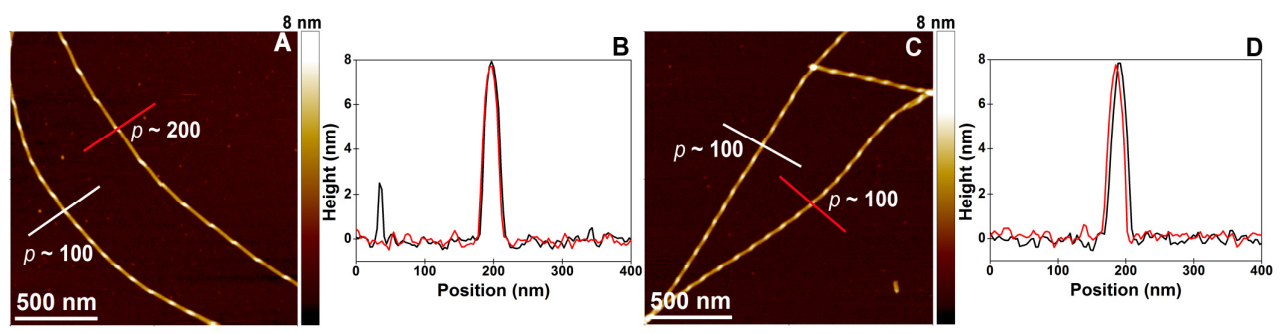

Figure 2.7 Representative AFM height images of wt $\alpha \operatorname{Syn}(A, C)$ and corresponding height profiles (B, D). Tapping mode AFM height images of wt $\alpha$ Syn fibrils prepared in $10 \mathrm{mM}$ Tris- $\mathrm{HCl}, 10 \mathrm{mM} \mathrm{NaCl}$ at pH 7.4 and in the absence (A) or presence (C) of 0.1 mM EDTA. White and red lines in (A) and (C) indicate positions of the line profiles depicted in (B) and (D). Labels $(100,150)$ signify approximate value for periodicity $(p)$ of the fibrils in $\mathrm{nm}$. AFM image size: $1.7 \times 1.7 \mu \mathrm{m}$.

$119 \pm 24 \mathrm{~nm}$ for fibrils prepared without EDTA (Figures 2.7 and 2.8 and Table 2.2). The height distribution of wt aSyn fibrils formed in the presence of EDTA however showed a slightly narrower distribution when compared to fibrils aggregated without EDTA. It is notable that only reducing the salt concentration from 10 to $1 \mathrm{mM} \mathrm{NaCl}$, in the absence of EDTA, makes the aggregations more homogeneous regarding morphology of the fibrils formed. The kinetics of fibrillization are however slower, and a smaller protein fraction is included in fibrils indicating that very low salt conditions are less efficient conditions for fibrillization.

\section{DTNB assay}

All fibrillization reactions with the A140C mutant included DTT as a reducing agent in order to inhibit intermolecular disulfide bond formation through the cysteine residue at position 140. Formation of cysteine dimers is a favored reaction, such that the protein aliquots thawed from $-80^{\circ} \mathrm{C}$ often showed dimer protein bands on native gels. A typical aggregation reaction starting with monomers, as probed by ThT fluorescence takes on average 7 days to reach the plateau, while DTT has a much shorter half-life. The halflife of DTT has been previously shown to be affected by the presence of free divalent metal ions which enhance its oxidation (Charrier and Anastasio 2012). Thus, in order to prevent oxidation of DTT by trace metal ions, $0.1 \mathrm{mM}$ EDTA was included in the aggregation reactions. The effect of EDTA on the half-life of DTT was probed by quantitating free thiols in the aggregation reaction by a DTNB assay. In the DTNB assay, DTNB [5, 5'-dithiobis-(2-nitrobenzoic acid)] reacts with free thiols in a 1:1 
stoichiometric ratio in aqueous solvents, forming 2-nitro-5-thiobenzoate (NTB $\left.{ }^{2-}\right)$ which is intense yellow in color and can be quantified by absorbance at $412 \mathrm{~nm}$ (Riener, Kada et al. 2002). In reactions without EDTA, the half-life of DTT was about 1 day whereas in the presence of $0.1 \mathrm{mM}$ EDTA the half-life increased significantly to more than 9 days (Figure 2.9A). A control experiment to check if presence of EDTA alone could bring about the colorimetric change in the DTNB assay showed negative results (data not shown).

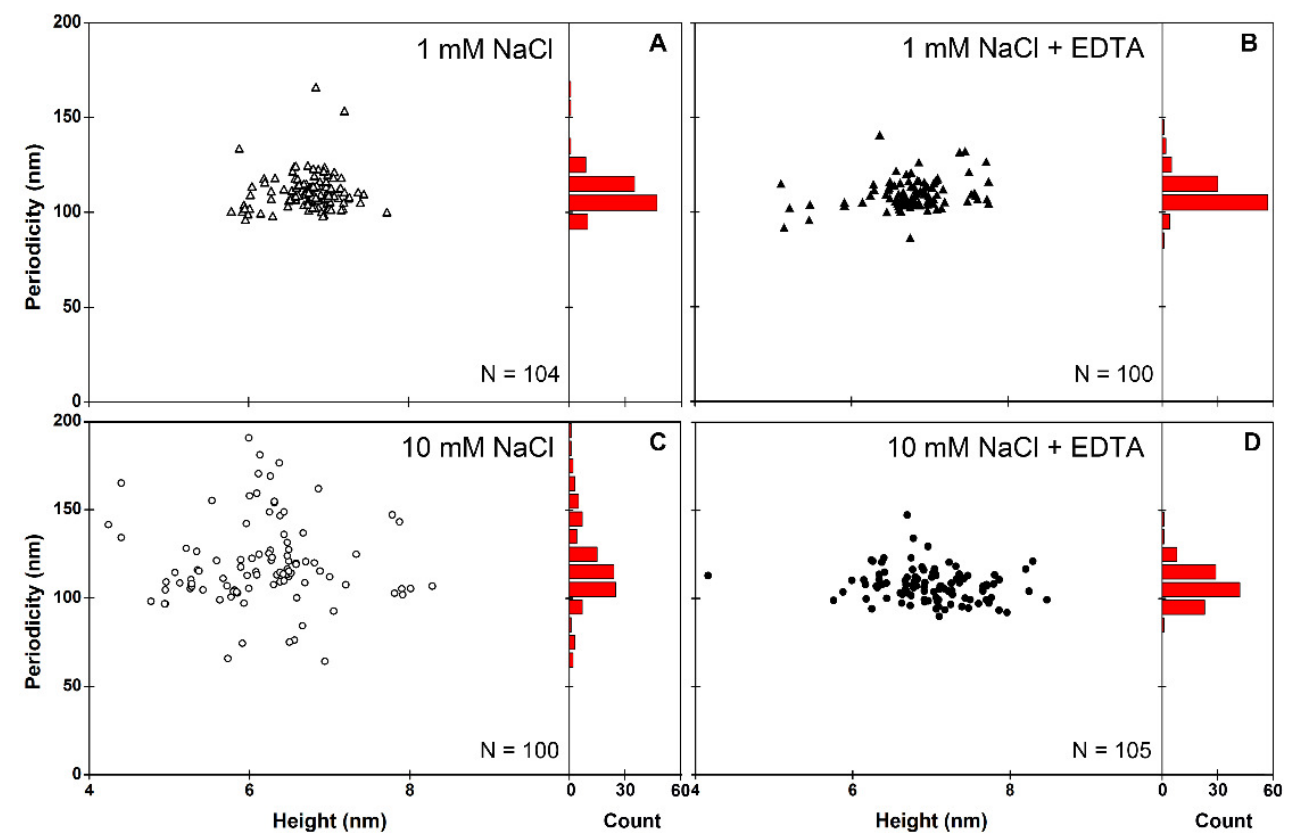

Figure 2.8 Scatter plots representing quantitative morphological features of wt $\alpha$ Syn fibrils prepared in $10 \mathrm{mM}$ Tris- $\mathrm{HCl}$ at $\mathrm{pH} 7.4$ with $1 \mathrm{mM}$ or $10 \mathrm{mM} \mathrm{NaCl}$ in the absence $(\mathrm{A}, \mathrm{C})$ or presence $(\mathrm{B}, \mathrm{D})$ of $0.1 \mathrm{mM}$ EDTA. Next to each scatter plot a histogram in red shows the distribution of periodicities for the fibrils. Bin size: $10 \mathrm{~nm}$. (A, C) Fibrils grown in the absence of EDTA display a broad distribution of fibril periodicities notably for reactions aggregated at $10 \mathrm{mM} \mathrm{NaCl}$. (B, D) Fibrils produced in the presence of EDTA show a narrow distribution of fibril periodicities. Fibril periodicities show no apparent correlation with heights in the absence $(A, C)$ or presence $(B, D)$ of EDTA. 

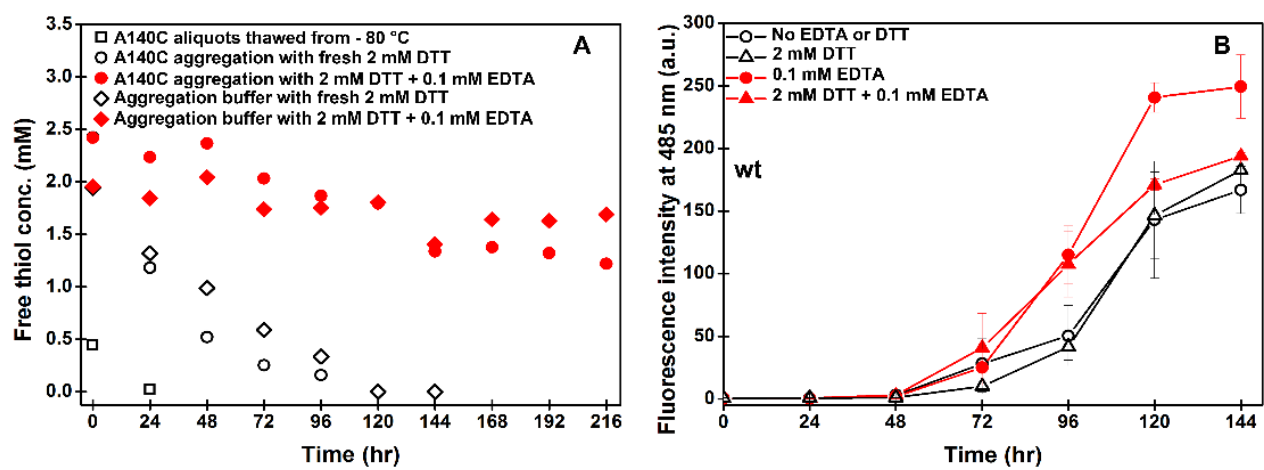

Figure 2.9 (A) DTNB assay probing free thiols as an indicator of the DTT half-life in the absence or presence of $0.1 \mathrm{mM}$ EDTA as a function of time. (B) Effect of reducing conditions on wt aSyn aggregation as measured by ThT fluorescence. Aggregation buffer included $10 \mathrm{mM}$ Tris- $\mathrm{HCl}, 10 \mathrm{mM}$ $\mathrm{NaCl}$ at $\mathrm{pH} 7.4$ with no EDTA or DTT/ $2 \mathrm{mM} \mathrm{DTT/} 0.1 \mathrm{mM}$ EDTA/ and $2 \mathrm{mM}$ DTT with $0.1 \mathrm{mM}$ EDTA. Error bars denote standard deviation among triplicates.

In the presence of EDTA, the notable increase in the half-life of DTT as well as the qualitative influence on fibrillization of aSyn is suggestive of two distinct possibilities. EDTA primarily acts as a chelating agent and sequesters the trace divalent metal ions present; as a result, it prevents oxidation of DTT in A140C fibrillization. Additionally, this chelating property possibly curbs interaction of metal ions with the negatively charged C-terminus of aSyn monomers, thereby altering its conformation to one favorable for aggregation (Uversky, Li et al. 2001; Hoyer, Cherny et al. 2004). Previous studies have shown that sequestration of metal ions by specific chelators leads to formation of more ordered fibrils, which have a sharper X-ray diffraction pattern owing to better alignment of the sample (Liu, Moloney et al. 2011). Evidence for enhancement of half-life of DTT and modulation of metal ion interaction with aSyn monomer being independent of each other is apparent in fibrillization of wt aSyn in the presence of EDTA but without DTT, where similar ThT kinetics could be observed (Figure 2.2B). Furthermore, wt aggregation experiments including DTT, to probe the effect of reducing conditions, did not show any decisive trend in the ThT assay (Figure 2.9B).

\section{Native PAGE analysis}

Native polyacrylamide gel electrophoresis (nPAGE) is sensitive to the conformations of protein molecules and can be insightful about the aggregating events. 


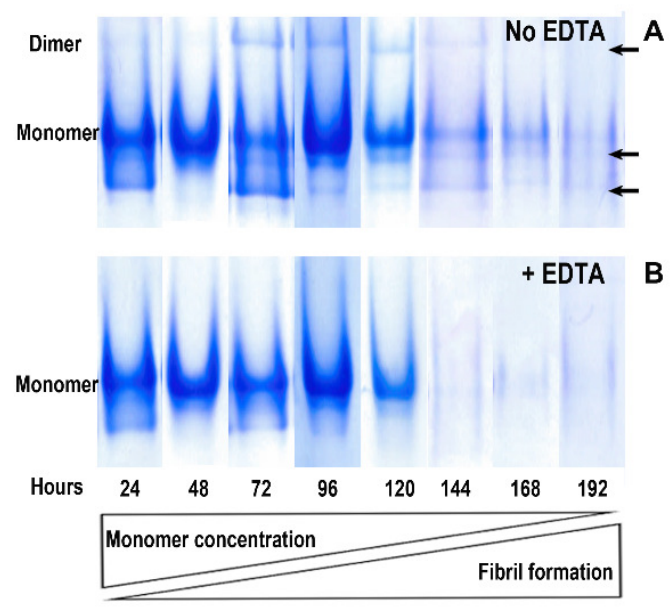

Figure 2.10 12\% clear native PAGE analysis of A140C aSyn fibrillization reactions. Aggregation reactions were set up in $10 \mathrm{mM}$ Tris- $\mathrm{HCl}, 10 \mathrm{mM} \mathrm{NaCl}, 2 \mathrm{mM}$ DTT at $\mathrm{pH} 7.4$ and in the absence (A) or presence (B) of $0.1 \mathrm{mM}$ EDTA. Lanes in panel (A) and (B) show qualitative differences in the active aggregation reactions as a function of time. Note the appearance of additional bands in the fractions without EDTA at 72 hours and later (indicated by black arrows). Monomer protein concentration decreases with time dependent accumulation of fibrillar species, which do not electrophorese in the polyacrylamide gel. Lanes in panel (A) and (B) are parts of separate gels electrophoresed for each time point.

A140C aggregation at different $\mathrm{NaCl}$ concentrations, with and without EDTA was followed on $12 \% \mathrm{nPAGE}$ for qualitative analysis of conformers and oligomeric assemblies. Initially, all the fractions exhibited a similar profile, but by 72 hours, concomitant with near depletion of DTT in reactions without EDTA, additional faster migrating bands (below the monomer band) were apparent together with a dimer band. However, in reactions with EDTA, no additional bands could be observed, and over time, monomer bands became fainter indicating exhaustion of the free monomers in the solution (Figure 2.10). The formation of additional faster migrating aSyn conformers appears to be prevented by DTT (half-life of which is critically influenced by EDTA) as they tend to appear only once DTT is exhausted in the solution. To further ascertain the role of EDTA as an enhancer of DTT's half-life in A140C fibrillization an aggregation with TCEP as reducing agent was done using $100 \mu \mathrm{M}$ A140C aSyn in 10 $\mathrm{mM}$ Tris- $\mathrm{HCl}, 10 \mathrm{mM} \mathrm{NaCl}, 2 \mathrm{mM}$ TCEP at $\mathrm{pH}$ 7.4. TCEP is a stronger and a more stable reducing agent than DTT and does not require EDTA. Fibrillization in the presence of TCEP showed faster aggregation kinetics in the ThT assay than reactions 
with DTT (+/- $0.1 \mathrm{mM}$ EDTA) (Figure 2.11A). Also on nPAGE no additional bands were observed during the aggregation thus confirming that the origin of these bands is most likely due to lack of reducing conditions after 72 hours in experiments with DTT (Figure 2.11B).

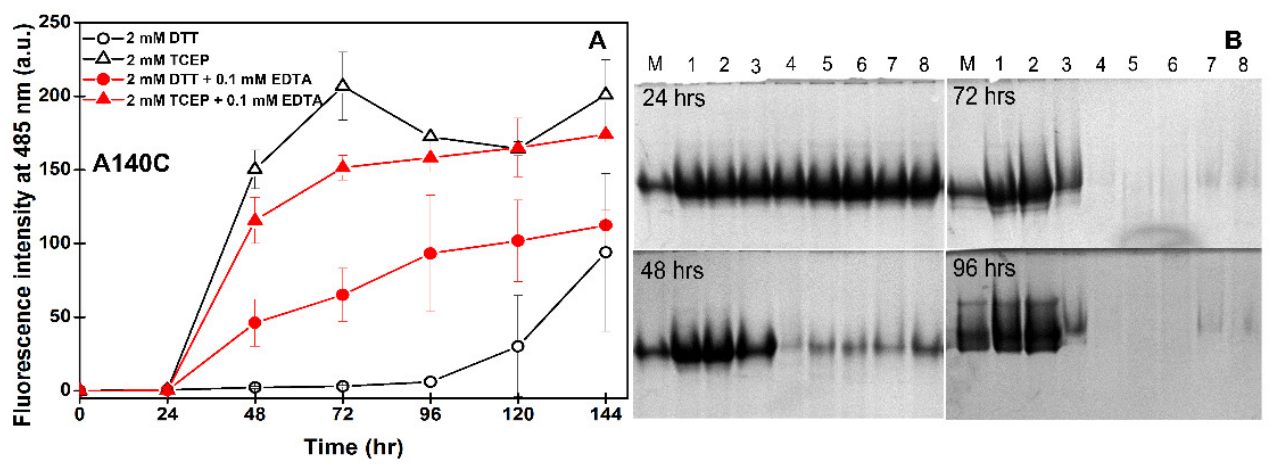

Figure 2.11 (A) Effect of TCEP as reducing agent on A140C aSyn aggregation as measured by ThT fluorescence. Aggregation buffer included $10 \mathrm{mM}$ Tris- $\mathrm{HCl}, 10 \mathrm{mM} \mathrm{NaCl}$ at $\mathrm{pH} 7.4$ with either $2 \mathrm{mM}$ DTT / 2 mM DTT with $0.1 \mathrm{mM}$ EDTA / $2 \mathrm{mM}$ TCEP/ $2 \mathrm{mM}$ TCEP with $0.1 \mathrm{mM}$ EDTA at pH 7.4. Error bars denote standard deviation among triplicates. (B) $12 \%$ clear native PAGE analysis of A140C aSyn aggregation with TCEP as reducing agent. Aggregation buffer included $10 \mathrm{mM}$ Tris- $\mathrm{HCl}, 10 \mathrm{mM}$ $\mathrm{NaCl}$ at $\mathrm{pH} 7.4$ with +/- TCEP or DTT and +/- EDTA. Lanes: M: Monomers, 1, 2: duplicates with 2 mM DTT; 3, 4: duplicates with $2 \mathrm{mM}$ DTT and $0.1 \mathrm{mM}$ EDTA; 5, 6: duplicates with $2 \mathrm{mM}$ TCEP; 7 , 8: duplicates with $2 \mathrm{mM}$ TCEP and $0.1 \mathrm{mM}$ EDTA at $\mathrm{pH}$ 7.4.

In wt fibrillization reactions (no DTT), followed on nPAGE, at times faint additional bands could be observed around 120 and 144 hours along with discernible differences in the monomer band intensity (Figure 2.12). Overall, the presence of additional bands as well as a more intense monomer band in EDTA free aggregation reactions at 72 hours and later are demonstrative of a lower fraction of monomers being incorporated into fibrils. 


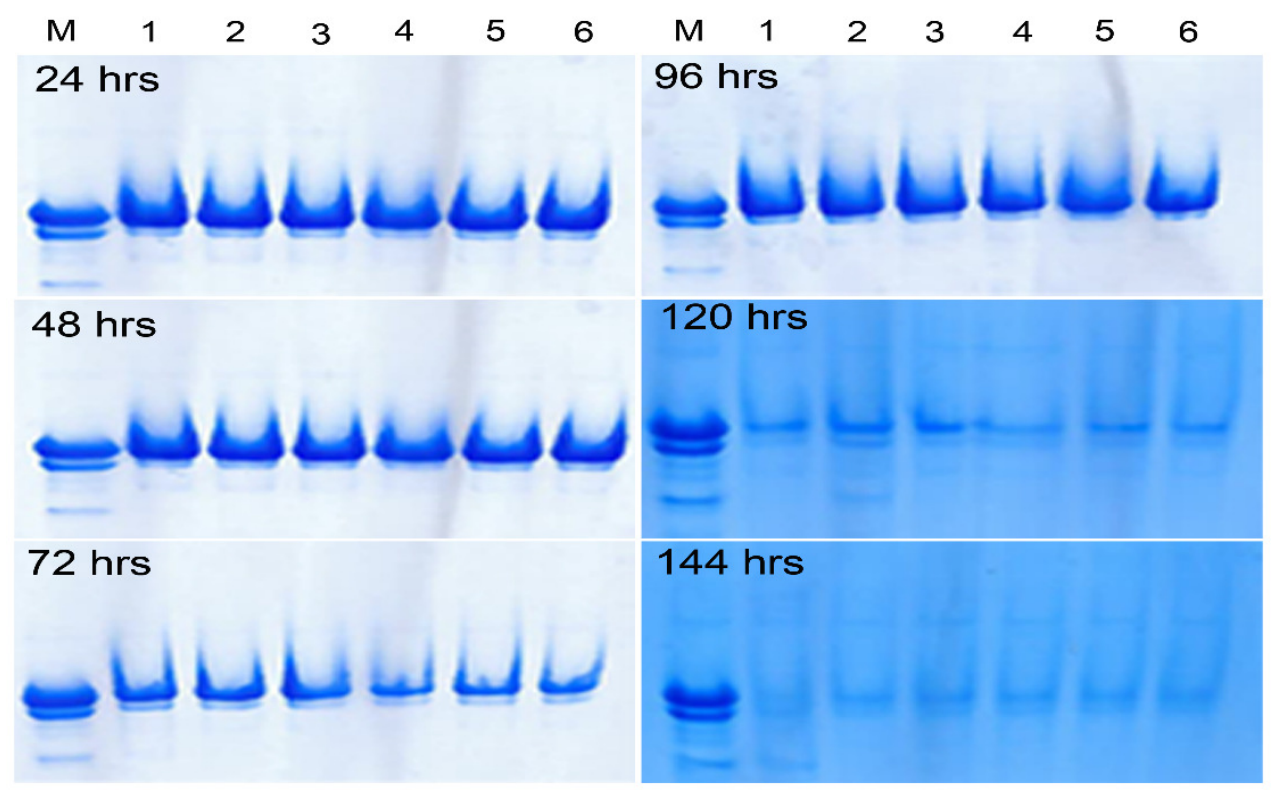

Figure 2.12 12\% clear native PAGE analysis of wt $\alpha$ Syn fibrillization reactions. Aggregation reactions were set up in $10 \mathrm{mM}$ Tris- $\mathrm{HCl}, 10 \mathrm{mM} \mathrm{NaCl}$ at $\mathrm{pH} 7.4$ and in the absence or presence of $0.1 \mathrm{mM}$ EDTA. Monomer protein concentration decreases with time dependent accumulation of fibrillar species, which do not electrophorese in the polyacrylamide gel. Faint additional bands could be observed around 120 and 144 hours along with discernible difference in the monomer band intensity. Lanes: $\mathrm{M}=$ wt monomers; $1,2,3$ : triplicates of wt aggregation without $0.1 \mathrm{mM} \mathrm{EDTA;} 4,5$, 6: triplicates of wt aggregation with $0.1 \mathrm{mM}$ EDTA.

\section{CONCLUSIONS}

The work presented in this chapter shows that fibrillization of aSyn can be carefully modulated by solution conditions to produce morphologically homogeneous populations of fibrils. Fibrillization in low salt conditions seems to reduce stochasticity inherent with amyloid fibril formation. Buffer conditions of $10 \mathrm{mM}$ Tris- $\mathrm{HCl}, 10 \mathrm{mM}$ $\mathrm{NaCl}, 2 \mathrm{mM}$ DTT and $0.1 \mathrm{mM}$ EDTA at $\mathrm{pH} 7.4$ yielded A140C aSyn fibrils displaying a narrow distribution of periodicities with an average value of $102 \pm 6 \mathrm{~nm}$; in similar solution conditions but without DTT, wt aSyn formed fibrils with an average periodicity of $107 \pm 9 \mathrm{~nm}$. Inclusion of EDTA is instrumental in forming fibrils with well-defined periodicity, by two likely effects involving its chelating function. Firstly, in 
reactions with thiol containing A140C aSyn, it reduces oxidation of DTT by metal ions, which significantly enhances DTT's half-life (thus preventing protein dimerization). Secondly, it prevents the interaction of metal ions with the negatively charged Cterminus of aSyn monomers potentially introducing a conformational bias that may positively influence the nucleation. ThT assays, RMC and nPAGE results support the proposition of more efficient incorporation of aSyn monomers into fibrils in the presence of $0.1 \mathrm{mM}$ EDTA.

In conclusion, the aggregation conditions described in this chapter appear optimal for producing homogeneous fibril samples. The present results are important for the use of fibrils in bio-nanomaterial applications as it enables us to prepare homogeneous and reproducible fibrils. However, so far we have discussed the effect of these aggregation conditions on A140C and wt aSyn fibrils only, sequences important for functionalization and in relation with sporadic $\mathrm{PD}$, respectively. It is interesting to speculate on the fibrillization behavior of other aSyn sequences at these aggregation conditions, notably the ones associated with familial forms of PD. If the disease related mutants also form homogeneous fibrils, it would be an excellent opportunity to study comparative longitudinal changes, if any.

\section{ACKNOWLEDGEMENTS}

We thank Kirsten van Leijenhorst-Groener, Yvonne Kraan and Nathalie Schilderink for protein expression and purification, and Dr. Martin Bennink, Kees van der Werf and Robert Molenaar for advice on AFM. 


\section{Chapter 3}

\section{Distinct mechanisms determine fibril morphology during growth and maturation of $\alpha$-synuclein}

Fibrillization of soluble amyloidogenic protein monomers usually follows a sigmoidal growth curve, with a lag phase, a growth phase and a plateau phase. The plateau phase in the aggregation reaction is believed to represent the equilibrium between the fibrillar, oligomeric and monomeric species. Moreover, the fibrils found at the plateau phase are often referred to as mature fibrils, implying completion of the self-assembly process. In this chapter, we study the changes in the morphology of fibrils formed from wt aSyn and its disease-related mutants (A30P, E46K, and A53T) during active fibrillization (late lag to plateau phase) and while in storage up to 1 year. All the sequences showed polymorphism during the late lag phase, while akin to the fibrils produced in chapter 2, wt, A30P and E46K formed homogeneous fibrils at the plateau phase. Furthermore, we find that the plateau phase does not necessarily reflect cessation of the aggregation process. The morphology of wt aSyn fibrils changes over a period of 6 months whereas in contrast the disease mutants form stable fibrils. The results presented in this chapter highlight that all aSyn sequences fibrillize by a competitive growth albeit single amino acid differences can have a significant effect on the maturation of the fibrils.

Parts of this chapter will be published as: Arshdeep Sidhu, Ine Segers-Nolten, Vincent Raussens, Mireille M. A. E. Claessens, Vinod Subramaniam. Distinct mechanisms determine a-synuclein fibril morphology during growth and maturation. 


\section{INTRODUCTION}

In familial form of $\mathrm{PD}$, the affected individuals are commonly shown to carry an autosomal dominant point mutation in the aSyn gene. The mutations known so far, A30P, E46K, H50Q, G51D, A53T and A53E are associated with enhanced aggregation (into oligomeric or fibrillar form) of the endogenous aSyn, potentially leading to PD (Polymeropoulos, Lavedan et al. 1997; Kruger, Kuhn et al. 1998; Zarranz, Alegre et al. 2004; Lesage, Anheim et al. 2013; Proukakis, Dudzik et al. 2013; Pasanen, Myllykangas et al. 2014). Of these mutations, we focus our study on A30P, E46K and A53T sequence mutations in this chapter. In general, the disease mutants, A30P, E46K and A53T, are believed to aggregate faster into aggregation intermediates or fibrils in comparison to wt aSyn. However, each sequence has been proposed to exhibit distinct aggregation kinetics and produce fibrils of different morphologies (Conway, Harper et al. 1998; Giasson, Uryu et al. 1999; Narhi, Wood et al. 1999; Li, Uversky et al. 2001; Choi, Zibaee et al. 2004; Greenbaum, Graves et al. 2005; van Raaij, Segers-Nolten et al. 2006; Ghosh, Mondal et al. 2013; Ghosh, Sahay et al. 2014).

De novo fibrillization of soluble amyloidogenic proteins typically follows a sigmoidal growth curve. The plateau phase in ThT fluorescence assays is often assumed to indicate the equilibrium in the fibrillization reaction. However, over the past decade various studies have suggested that the plateau phase is not a true equilibrium in a fibrillization reaction and that slow but significant rearrangement of fibrils takes place over time (Vilar, Chou et al. 2008; Morel, Varela et al. 2010; Guilliams, El-Turk et al. 2013; Ma, Komatsu et al. 2013; Nystrom, Psonka-Antonczyk et al. 2013). Morphological analysis of fibrils by AFM and EM at plateau phase usually shows polymorphic fibrils (Jimenez, Nettleton et al. 2002; Khurana, Ionescu-Zanetti et al. 2003; Heise, Hoyer et al. 2005; Kodali and Wetzel 2007; Fandrich, Meinhardt et al. 2009; Pellarin, Schuetz et al. 2010). Detailed NMR studies suggest differences in the backbone conformation, electrostatic interactions and salt bridges in different polymorphs of $A \beta$ and $\alpha$ Syn (Heise, Hoyer et al. 2005; Petkova, Leapman et al. 2005; Paravastu, Leapman et al. 2008; Bousset, Pieri et al. 2013; Gath, Bousset et al. 2014). Thus, morphological polymorphism is in all likelihood a consequence of variations in the molecular structure, which in turn arises from multiple conformations accessible to the soluble unfolded protein monomer.

The multiple conformations, based on the energy landscape theory for protein 
folding, could be due to a rough funneled landscape that allows multiple low-energy conformations (local energy minima) in the early stages of folding (Dill and Chan 1997; Onuchic, Luthey-Schulten et al. 1997; Dobson 2003). In IDPs particularly, the energy difference between the thermodynamically stable conformations (depth of the well) and the kinetic barriers, for conversion to another conformation, (height of the barrier) are proposed to be very low in the absence of a binding partner (Gershenson, Gierasch et al. 2014). The conformational dynamics of IDPs are thus more diverse than the globular proteins. The multiple local energy minima are suggested to act as kinetic traps that stabilize partially folded or misfolded conformations (Guo and Thirumalai 1995; Eaton, Thompson et al. 1996). Such metastable conformations can act as an intermediate state towards the aggregated form of the protein rather than the native, soluble state (Jahn, Parker et al. 2006). The accessibility of the local energy minima associated with different amyloid conformations are proposed to be critically influenced by the aggregation conditions and the protein sequence (Nielsen, Khurana et al. 2001; Hoyer, Antony et al. 2002; Jimenez, Nettleton et al. 2002; Raman, Chatani et al. 2005; Fink 2006; Morel, Varela et al. 2010; Buell, Galvagnion et al. 2014). Point mutations in a protein sequence most likely affect the energy landscape resulting in characteristic aggregation behavior. Accordingly, in aSyn aggregations, wt and disease-related mutation sequences are suggested to exhibit distinct aggregation kinetics and morphologies (Conway, Harper et al. 1998; Giasson, Uryu et al. 1999; Narhi, Wood et al. 1999; Li, Uversky et al. 2001; Choi, Zibaee et al. 2004; Greenbaum, Graves et al. 2005; van Raaij, Segers-Nolten et al. 2006; Ghosh, Mondal et al. 2013; Ghosh, Sahay et al. 2014)

In addition to the proposed differences in the conformation of the proteins leading to distinct fibril morphologies, the polymorphs have been suggested to exhibit different levels of toxicity in cell based studies (Petkova, Leapman et al. 2005; Bousset, Pieri et al. 2013; Peelaerts, Bousset et al. 2015). Given the observed toxicity profile of distinct amyloid polymorphs, a growing number of studies compare the polymorphic behavior of amyloids to the strain-like properties of prions (Petkova, Leapman et al. 2005; Bousset, Pieri et al. 2013; Goedert 2015; Peelaerts, Bousset et al. 2015). Like prions, these polymorphs show nucleation (nucleated polymerization behavior), templating (seed aggregations) and cell-to-cell transfer (Petkova, Leapman et al. 2005; Bousset, Pieri et al. 2013; Dunning, George et al. 2013; George, Rey et al. 2013; Guo, Covell et al. 2013; Goedert, Falcon et al. 2014; Peelaerts, Bousset et al. 2015). Studies on transmission of 
multiple system atrophy pathology in a transgenic mouse model with complete sets of clinical and biochemical markers provide compelling evidence for the prion-like behavior of aSyn (Watts, Giles et al. 2013). However, not much data are available for the competitive growth of different polymorphs in aSyn or other amyloid systems. Competitive growth between prion strains is a hallmark for phenotype determination and is thus one of the critical factors determining the physiological effect in vivo (Dickinson, Fraser et al. 1975; Tanaka, Collins et al. 2006; Shikiya, Ayers et al. 2010). Longitudinal in vitro studies assessing the relative ratios of the polymorphs over time are valuable to understand the dynamic relation between the experimentally observed morphologies.

In the present chapter, applying the aggregation conditions optimized in chapter 2, we prepare homogeneous fibril samples of wt, A30P and E46K aSyn at the plateau phase. Using homogeneity of the fibril morphology at the plateau phase as a reference, we systematically compare the long-term changes in the fibril morphology of the aSyn sequences at single fibril level using AFM. The morphology of the helical fibrils was quantitatively studied by measuring their heights and periodicities over a period of 6 months, supplemented with qualitative data at 1 year. Our study provides insight into the changes in the periodicities of the selected aSyn sequences during fibrillization and while in storage.

\section{MATERIALS AND METHODS}

\section{Expression and purification of $\alpha$ Syn}

Wt and disease mutants (A30P, E46K and A53T) of aSyn were used in the present study. All the proteins were expressed and purified as described elsewhere (Sidhu, SegersNolten et al. 2014).

\section{Fibrillization assay and ThT fluorescence}

$250 \mu \mathrm{l}$ of monomeric stocks of wt and disease mutant $\alpha$ Syn, stored at $-80{ }^{\circ} \mathrm{C}$, were thawed. Aggregation reactions for all the proteins were set up at $100 \mu \mathrm{M} \alpha$ Syn in $10 \mathrm{mM}$ Tris- $\mathrm{HCl}, 10 \mathrm{mM} \mathrm{NaCl}$ and $0.1 \mathrm{mM}$ EDTA, at $\mathrm{pH}$ 7.4. All reactions were prepared in triplicate with volumes of $400 \mu \mathrm{l}$ each in $2 \mathrm{ml}$ Lo-Bind round bottom Eppendorf centrifuge tubes and were incubated at $37^{\circ} \mathrm{C}$ with $500 \mathrm{rpm}$ orbital shaking in an Eppendorf Thermo-mixer comfort. Progress of fibrillization was followed by a ThT 
fluorescence assay as described elsewhere (Sidhu, Segers-Nolten et al. 2014). Fibrils prepared at $4{ }^{\circ} \mathrm{C}$ were grown with same the protocol but with the Thermo-mixer placed in a cold room at $4{ }^{\circ} \mathrm{C}$.

\section{Atomic force microscopy (AFM)}

AFM samples were prepared by adsorbing $10 \mu \mathrm{l}$ of 5-20 times diluted aggregation samples on freshly cleaved mica (Muscovite mica, V-1 quality, EMS) for 4 minutes, followed by 2 gentle washes with $100 \mu \mathrm{l}$ of fresh Milli-Q water and drying in a gentle stream of nitrogen gas. AFM images were acquired on a Bioscope Catalyst (Bruker) in soft tapping mode in air using a silicon probe, NSC36 tip B with force constant of 1.75 $\mathrm{N} / \mathrm{m}$ (NanoAndMore). All images were captured with a resolution of $512 \times 512$ pixels per image with a scan rate of $0.5 \mathrm{~Hz}$. For each solution condition about 100 nonoverlapping fibrils were qualitatively analyzed by Scanning Probe Image Processor-6.02 (Image Metrology) software; for quantitative analysis of height $(h)$ and periodicity $(p)$ a custom written Matlab script using DIPimage toolbox (version 2.3) was used (Faas, Rieger et al. 2009). The script is based on quantitative analysis of AFM images as described elsewhere (van Raaij, Segers-Nolten et al. 2006). To ensure reproducibility in the observed results, for all samples at a given time point, three independent aggregations were done in triplicate and about 30 images were acquired from one of the triplicates from each aggregation.

\section{Dot blot analysis}

A dot blot analysis was done to probe degradation of aSyn fibrils stored for 1 year (matured fibrils). $100 \mu \mathrm{l}$ of aggregation reactions were centrifuged at $21,000 \times \mathrm{g}$ for 30 minutes in an IEC Micromax microcentrifuge (Thermo Fisher Scientific). $80 \mu$ of the supernatant was discarded and the pellet was resuspended in the rest of the $20 \mu \mathrm{lbuffer}$ (10 mM Tris- $\mathrm{HCl}, 10 \mathrm{mM} \mathrm{NaCl}$ at $\mathrm{pH}$ 7.4). Immobilon-P PVDF membrane (Millipore) was wetted in $100 \%$ methanol for 15 seconds and rinsed in Milli-Q water. $5 \mu$ of each sample was spotted on the wet membrane and the membrane was soaked in $100 \%$ methanol for 10 seconds. The blot was air dried for 2 hours followed by re-wetting of the membrane for 5 seconds. The membrane was fixed in $0.4 \%$ PFA (paraformaldehyde solution) in PBS at $\mathrm{pH} 7.4$ for 15 minutes at room temperature. Next, it was rinsed 3 times in PBS, at $\mathrm{pH}$ 7.4. The blot was blocked in $5 \%$ nonfat dry milk in 
TBS-T [Tris-buffered saline-Tween (50 mM Tris, $150 \mathrm{mM} \mathrm{NaCl}$ at $\mathrm{pH}$ 7.5-0.2 \% v/v Tween 20)] for 30 minutes and rinsed in TBS-T 3 times. Mouse IgG1 anti-aSyn 15-123 (610786, BD Biosciences) and Mouse monoclonal anti-aSyn 121-125 (sc-12767, Santa Cruz Biotechnology) antibodies were diluted (1'2000 and 1'1000, respectively) in $1 \%$ nonfat dry milk in TBS-T and incubated overnight at $4{ }^{\circ} \mathrm{C}$. The blot was washed 3 times in TBS-T for 5 minutes each and incubated with anti-mouse HRP conjugated secondary antibody (G21234, Invitrogen) (diluted: 1'8000) in TBS-T for 45 minutes at room temperature. The blot was washed 3 times in TBS and the immunoreactive dots were detected using Enhanced chemi-luminescence (Super Signal West Pico, Thermo Scientific) followed by imaging on a Fluor Chem M imager (ProteinSimple).

\section{ATR-FTIR experiment}

Attenuated total reflectance-Fourier transform infrared spectroscopy (ATR-FTIR) was done to investigate the structural features of plateau phase and 1 year old (matured) aSyn fibrils. Samples were prepared by centrifugation of $100 \mu \mathrm{l}$ of aggregation reactions at $21,000 \times \mathrm{g}$ for 30 minutes in an IEC Micromax microcentrifuge (Thermo Fisher Scientific). $80 \mu \mathrm{l}$ of the supernatant was discarded and the pellet was resuspended in 20 $\mu \mathrm{l}$ buffer (10 mM HEPES at pH 7.4). The ATR-FTIR spectra were acquired on a Bruker Equinox 55 spectrophotometer (Bruker Optics) equipped with a Golden Gate reflectance accessory (Specac). The internal reflection element was a diamond crystal (2 $\times 2 \mathrm{~mm}$ ) with an aperture angle of $45^{\circ}$ that yielded a single internal reflection. The crystal was cleaned with absolute ethanol and 2 to $4 \mu \mathrm{l}$ of sample was applied to the diamond crystal. The sample was dried with a gentle flow of nitrogen gas. Excess salt was removed by placing $10 \mu \mathrm{l}$ of Milli-Q water on top of the sample and removing excess water with a micropipette. The sample was dried again with a gentle flow of nitrogen gas. 128 co-added spectra were collected for each sample with a resolution of $2 \mathrm{~cm}^{-1}$. For data analysis, baseline correction was done by a straight line passing through ordinates $1753,1712,1593,1482,1425 \mathrm{~cm}^{-1}$, followed by water vapor subtraction. Then Fourier self-deconvolution was applied using a Lorentzian and Gaussian line shape for deconvolution and apodization, respectively. The identified peaks were curve fitted on non-deconvoluted spectra. Area under each deconvoluted peak was quantified to estimate the secondary structure of the sample (Goormaghtigh, Raussens et al. 1999). 


\section{Residual monomer concentration (RMC) and reversibility analysis}

Determination of the fraction of monomers left in the aggregation reaction was done by centrifugation of $100 \mu \mathrm{l}$ aliquots of aggregation reactions at $21,000 \times \mathrm{g}$ for 1 hour in an IEC Micromax microcentrifuge (Thermo Fisher Scientific). $80 \mu \mathrm{l}$ of the supernatant was aspirated and analyzed for RMC. Absorbances at 280 and $330 \mathrm{~nm}$ were measured from $2 \mu \mathrm{l}$ samples on a NanoDrop ND-1000 spectrophotometer (Isogen Life Science). The absorption values at $280 \mathrm{~nm}$ were corrected for scattering contributions before calculating the residual monomer concentration, using an extinction coefficient of 5600 $\mathrm{M}^{-1} \cdot \mathrm{cm}^{-1}$ (Grimsley 2004). For reversibility analysis, for each sample, $100 \mu \mathrm{l}$ of the plateau phase reaction was centrifuged at $21,000 \times \mathrm{g}$ for 1 hour and $80 \mu \mathrm{l}$ of the supernatant was removed. RMC was determined as mentioned above. The fibril pellet was resuspended in $80 \mu \mathrm{l}$ of fresh buffer (total volume $100 \mu \mathrm{l}$ ) of $10 \mathrm{mM}$ Tris- $\mathrm{HCl}, 10$ $\mathrm{mM} \mathrm{NaCl}$ and $0.1 \mathrm{mM}$ EDTA, at $\mathrm{pH} 7.4$ and stored at $37^{\circ} \mathrm{C}$ for 1 week. The procedure was repeated 3 times, while storing the sample at $37^{\circ} \mathrm{C}$ and 3 times while storing the sample at $4{ }^{\circ} \mathrm{C}$. At every alternate week $5 \mu$ of the fibril sample was analyzed by ThT fluorescence assay for detection of $\beta$-sheet positive fibrils.

\section{RESULTS AND DISCUSSION}

\section{Fibrillization of wt and disease mutants}

Wt $\alpha$ Syn and the A30P, E46K, A53T disease mutants were aggregated from monomers in uniform solution conditions of $10 \mathrm{mM}$ Tris- $\mathrm{HCl}, 10 \mathrm{mM} \mathrm{NaCl}, 0.1 \mathrm{mM}$ EDTA at $\mathrm{pH}$ 7.4, and the fibril formation was followed by a ThT fluorescence assay. The given solution conditions, as described in chapter 2, result in the formation of a homogeneous pool of fibrils at the plateau phase in the ThT assay for wt and A140C mutant aSyn. In a number of solution conditions the disease mutants of aSyn have been shown to aggregate faster than the wt (Conway, Harper et al. 1998; Narhi, Wood et al. 1999; Greenbaum, Graves et al. 2005). In our study A53T fibrillized faster than other aSyn sequences (Figure 3.1). The lag time was 1-2 days for A53T, A30P and E46K, while for wt aSyn it was 2-3 days. Correspondingly, the disease mutants reached the plateau phase after 4 (A30P and E46K) or 6 days (A53T), while wt $\alpha$ Syn took around 7 days to reach the plateau. 


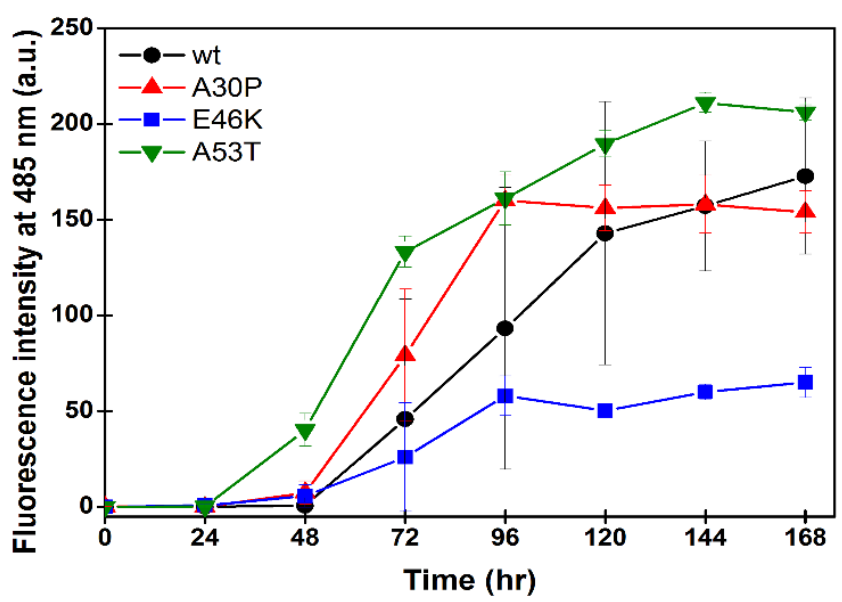

Figure 3.1 Comparison of aSyn (wt and disease mutants) aggregation kinetics measured by ThT fluorescence. Aggregation buffer includes $10 \mathrm{mM}$ Tris- $\mathrm{HCl}, 10 \mathrm{mM} \mathrm{NaCl}$ and $0.1 \mathrm{mM}$ EDTA at $\mathrm{pH}$ 7.4. Error bars denote standard deviation among triplicates.

\section{Fibril morphology over time}

The aggregations were performed at $37^{\circ} \mathrm{C}$ with shaking and were stored in quiescent conditions at $4{ }^{\circ} \mathrm{C}$ after reaching the plateau phase. Storage at $37^{\circ} \mathrm{C}$ (without shaking) led to formation of viscous gel-like networks in about $60 \%$ of the samples, most likely due to enhanced inter-fibrillar interactions (Semerdzhiev, Dekker et al. 2014). Highly viscous samples show clustered fibrils even on dilution, which cannot be analyzed at single fibril level by AFM impeding the study of long-term changes. Aliquots of fibril samples were drawn at different time points for AFM imaging. For quantitative morphological analyses of height and periodicity, samples were prepared at 4 time points based on the ThT fluorescence assay; at the end of the lag phase or early fibrillar phase ( 2 and 3 days for mutants and wt $\alpha$ Syn, respectively) to study the morphology of the early fibrillar species, at the plateau phase ( 5 and 7 days for mutants and wt aSyn, 


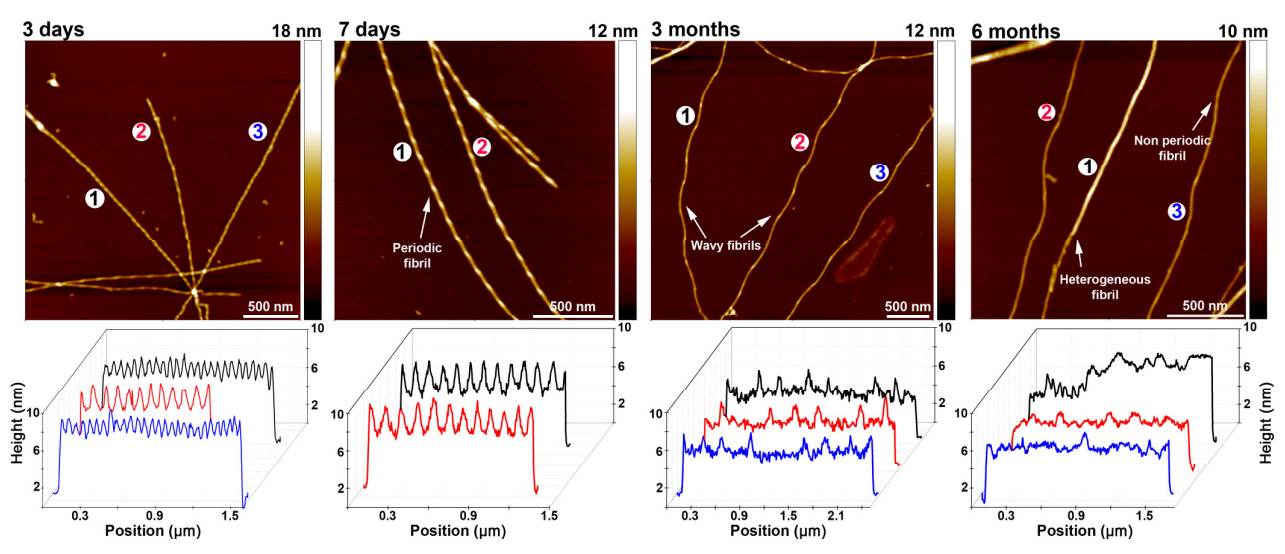

Figure 3.2 Upper panel: Representative AFM height images of wt aSyn fibrils at different time points. Lower panel: Color-coded longitudinal cross sections of the numbered fibrils in the AFM images. The cross sections depict the periodicities of the selected fibril. Sample from late lag phase (3 days) shows fibrils with different periodicities. However, fibrils at plateau phase ( 7 days) have similar periodicities. Fibrils at 3 months exhibit larger periodicities with distinct waviness, while samples of 6 months show non periodic fibrils and fibrils associated with other fibrils termed as heterogeneous fibrils.

respectively), and after 3 months and 6 months to assess the morphology changes of the fibrils during storage. Figure 3.2 shows typical morphology of the wt fibrils observed over time.

\section{Early fibril samples show polymorphism}

Samples imaged at $2 / 3$ days by AFM showed long fibrils for wt, A30P and A53T, while E46K fibrils were shorter and fewer in number. Further quantitative analyses of the AFM images revealed a broad distribution of periodicities of the helical fibrils for all samples (Figures 3.3 and 3.4: 2/3 days and Table 3.1). Interestingly in our study, none of the samples showed protofibrils, which, in accordance with the proposed hierarchical assembly model (HAM) (Khurana, Ionescu-Zanetti et al. 2003), should display fibrils of half heights and periodicities in comparison to mature fibrils. For all the samples, the early fibrillar species showed a distribution of heights and periodicities that were not correlated (Figure 3.4: 2/3 days). Wt $\alpha$ Syn fibrils showed two distinct groups of fibrils with periodicities around 50 and $100 \mathrm{~nm}$ and possibly a third fibril species with a periodicity of around $150 \mathrm{~nm}$ (Figures 3.2, 3.3 and 3.4). A30P mutant fibrils did not show any distinct peak in the periodicity distribution, but yielded a rather broad distribution from 50 to $150 \mathrm{~nm}$. E46K fibrils had one dominant population with 
periodicity around $80 \mathrm{~nm}$ but also displayed a broad distribution up to $320 \mathrm{~nm}$ in periodicity. A53T fibrils at 2 days were grouped in three categories; a) periodic fibrils: these fibrils showed a distinct modulation along their lengths with a periodicity of about $350 \mathrm{~nm}$; b) heterogeneous fibrils: these fibrils appeared associated with one or more fibril fragment(s) along their length resulting in an irregular appearance and hampering periodicity analysis; c) non-periodic fibrils: these fibrils showed no periodicity up to about $1 \mu \mathrm{m}$ of length (Figures 3.3, 3.4 and 3.5). Another notable feature in A53T aggregations was the presence of wavy fibrils at the end of the lag phase that showed undulation along the fibril length in contrast to the usually straight fibrils (Figures 3.2 and 3.5). Wt and A30P aSyn yielded $100 \%$ periodic fibrils, E46K yielded $94 \%$ periodic fibrils and A53T had $62 \%$ periodic fibrils in the early fibril samples (Table 3.2). The mean fibril height in all the samples varied between 5.7 and $6.7 \mathrm{~nm}$ (Figure 3.6 and Table 3.3: $2 / 3$ days). Overall, fibrils from all four sequences show appreciable morphological polymorphism at the late lag phase.

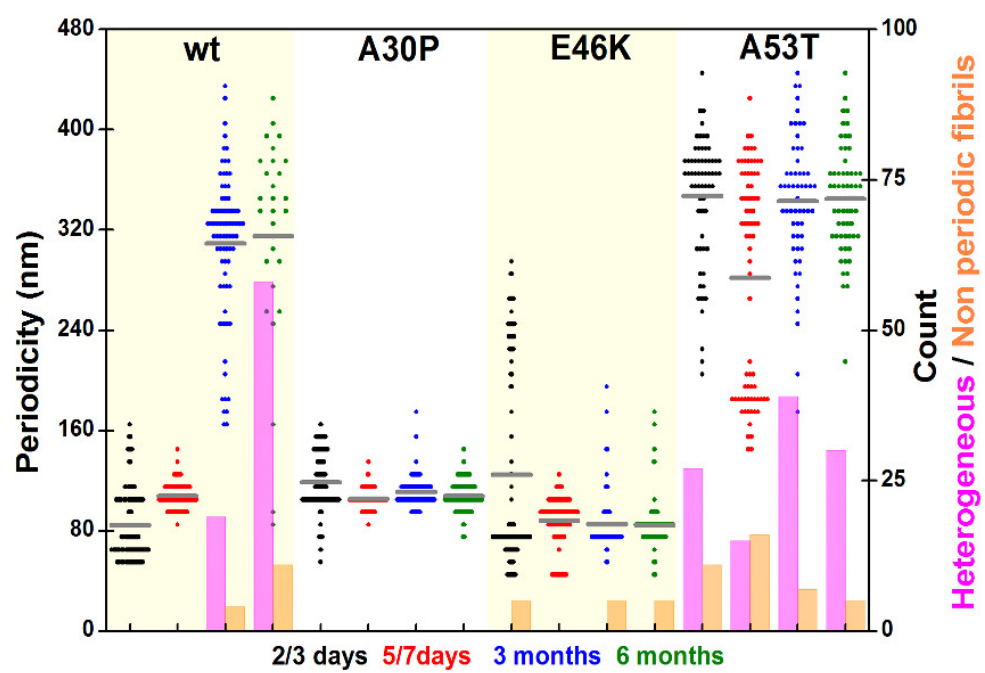

Figure 3.3 Box plot showing periodicities of wt and disease mutants of aSyn over 6 months. All the samples were aggregated in $10 \mathrm{mM}$ Tris- $\mathrm{HCl}, 10 \mathrm{mM} \mathrm{NaCl}$ and $0.1 \mathrm{mM}$ EDTA at $\mathrm{pH}$ 7.4. Bars in grey represent the mean of the distribution. Pink and orange bars indicate the content of heterogeneous and non-periodic fibrils. 

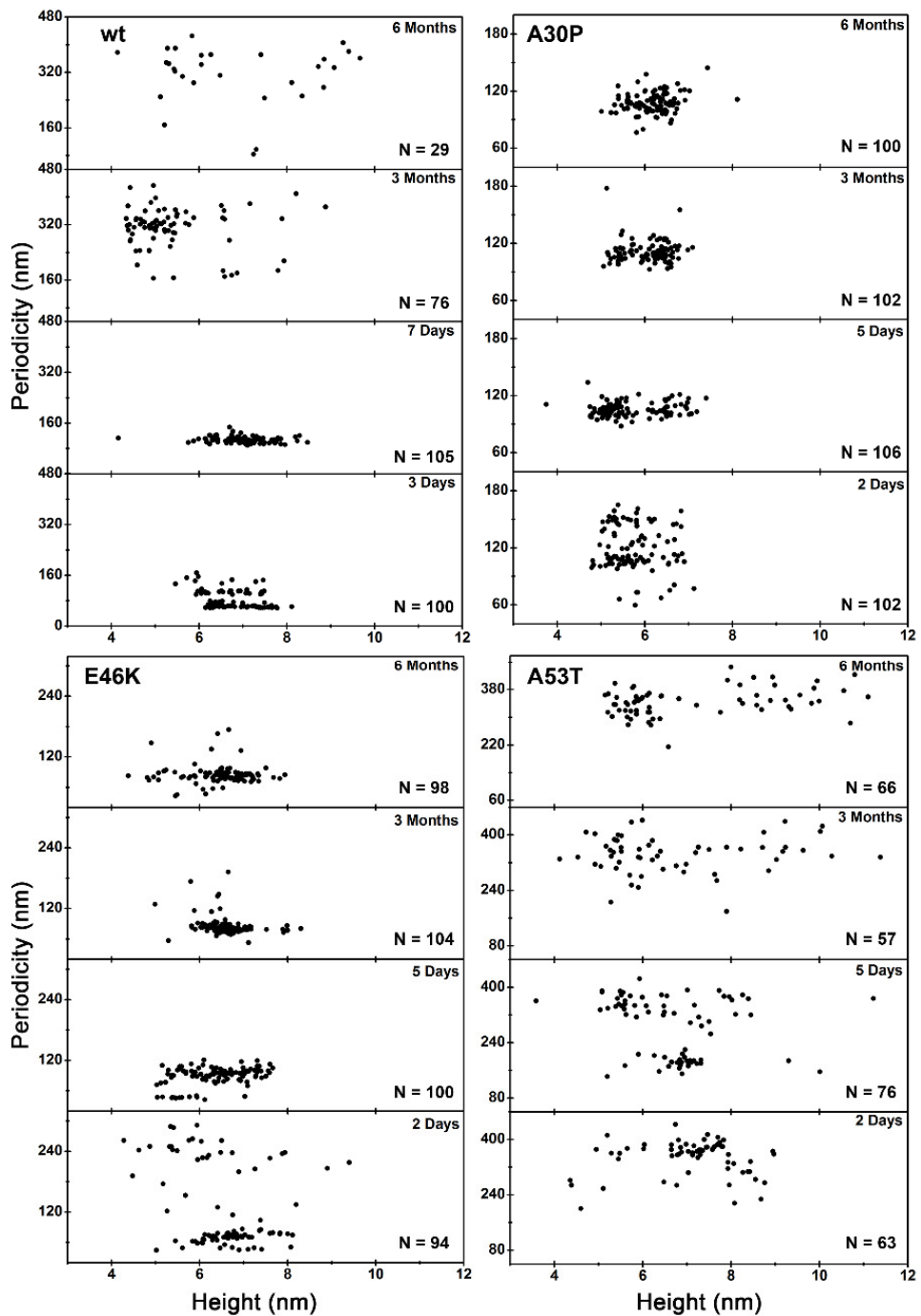

Figure 3.4 Scatter plots with heights and periodicities of periodic wt aSyn and disease mutant (A30P, E46K and A53T) fibrils. 2/3 days represent fibrils at the end of lag phase; 5/7 days represent fibrils at the plateau phase and at 3 and 6 months represent height and periodicities of stored fibrils. In all about 100 fibrils were imaged for each time point. $\mathrm{N}$ denotes the number of periodic fibrils plotted. Fibrils with no periodicity were categorized as heterogeneous or nonperiodic and are not included in this analysis. 
Table 3.1 Summary of mean fibril periodicity of wt aSyn and disease mutant fibrils over time ( \pm stdv) in nm. Fibrils with no periodicity are categorized as heterogeneous or non-periodic and are not included in this analysis.

\begin{tabular}{lcccc}
\hline & wt & A30P & E46K & A53T \\
\hline 2/3 Days & $84 \pm 28$ & $118 \pm 23$ & $124 \pm 80$ & $347 \pm 50$ \\
5/7 Days & $107 \pm 9$ & $105 \pm 7$ & $88 \pm 19$ & $282 \pm 87$ \\
3 Months & $309 \pm 60$ & $110 \pm 11$ & $85 \pm 19$ & $342 \pm 53$ \\
6 Months & $315 \pm 83$ & $107 \pm 11$ & $84 \pm 19$ & $344 \pm 40$ \\
\hline
\end{tabular}
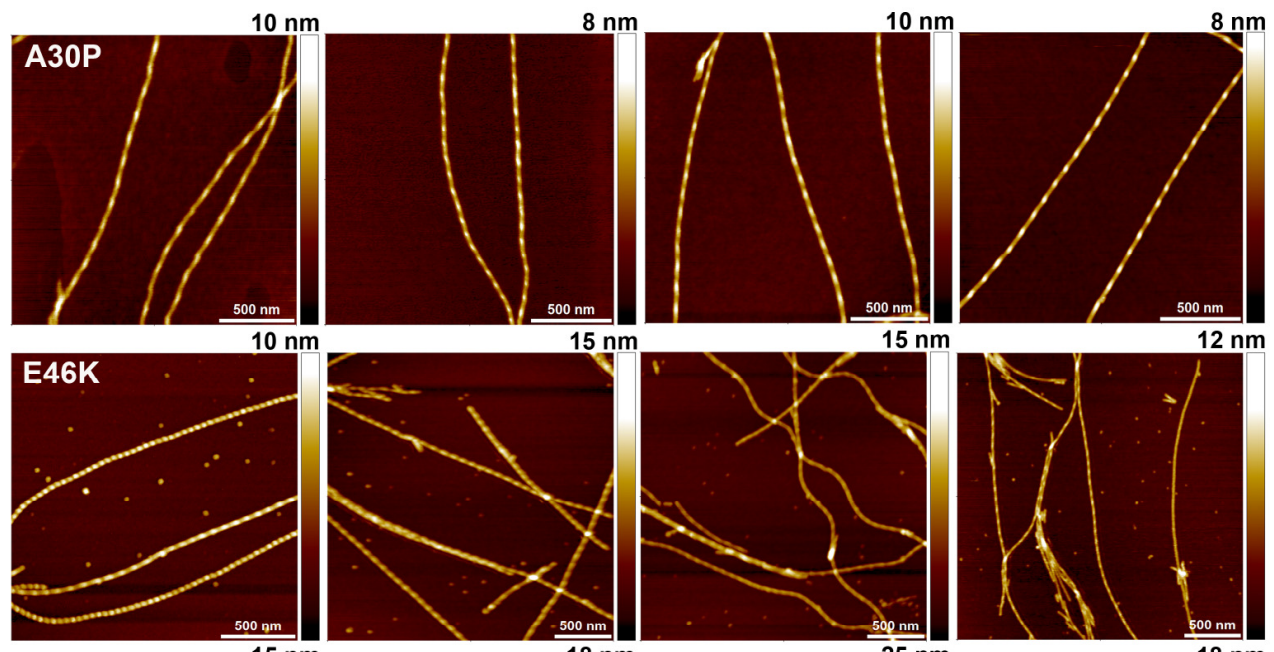

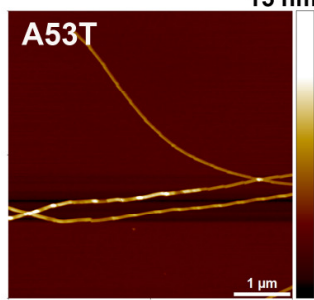

2 days

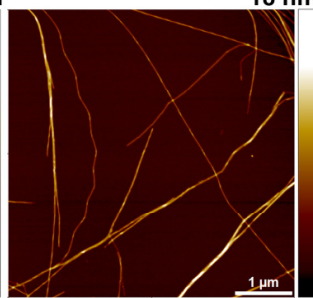

5 days

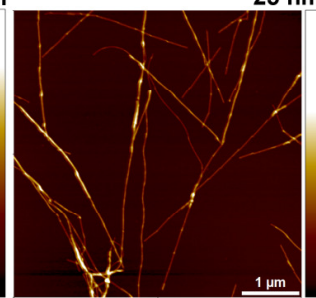

3 months

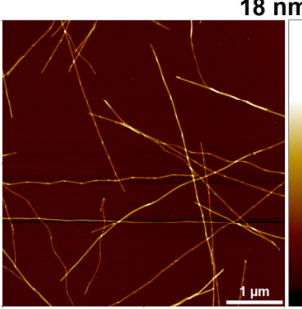

6 months

Figure 3.5 Representative AFM height images of fibrils from aSyn disease mutants (A30P, E46K, and A53T) over 6 months. Fibrils from each disease mutant show a distinct morphology that does not change over time. Fibrils were aggregated in $10 \mathrm{mM}$ Tris- $\mathrm{HCl}, 10 \mathrm{mM} \mathrm{NaCl}$ and, $0.1 \mathrm{mM}$ EDTA at $\mathrm{pH}$ 7.4. Samples for 2 and 5 days were prepared based on the ThT fluorescence assay. Image size: 1.70 $\times 1.70 \mu \mathrm{m}(\mathrm{A} 30 \mathrm{P}$ and $\mathrm{E} 46 \mathrm{~K}), 2.5 \times 2.5 \mu \mathrm{m}(\mathrm{A} 53 \mathrm{~T})$. 
Table 3.2 Summary of percentage of fibrils with periodicity in wt aSyn and disease mutant samples over time. Number of fibrils analysed for each time point is shown in parentheses.

\begin{tabular}{ccccc}
\hline & wt & A30P & E46K & A53T \\
\hline 2/3 Days & $100 \%(98)$ & $100 \%(100)$ & $94 \%(100)$ & $62 \%(101)$ \\
5/7 Days & $100 \%(105)$ & $100 \%(102)$ & $100 \%(100)$ & $71 \%(107)$ \\
3 Months & $76 \%(99)$ & $100 \%(106)$ & $95 \%(109)$ & $55 \%(103)$ \\
6 Months & $29 \%(98)$ & $100 \%(102)$ & $95 \%(103)$ & $65 \%(101)$ \\
\hline
\end{tabular}

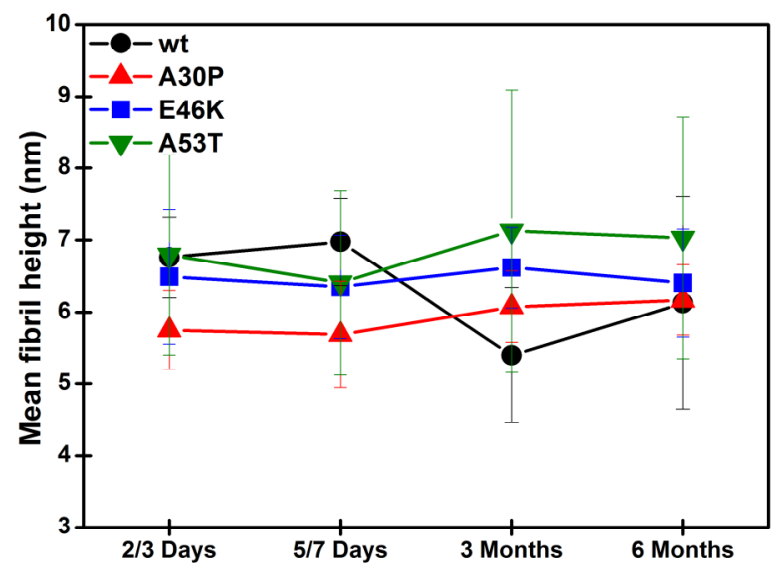

Figure 3.6 Mean fibril height of wt $\alpha$ Syn and disease mutant fibrils over time. The mean fibril height of the fibrils does not change significantly over a period of 6 months. Error bars denote standard deviation among triplicates.

Table 3.3 Summary of mean fibril height of wt aSyn and disease mutant fibrils over time ( \pm stdv) in nm.

\begin{tabular}{lcccc}
\hline & wt & A30P & E46K & A53T \\
\hline 2/3 Days & $6.7 \pm 0.5$ & $5.7 \pm 0.5$ & $6.4 \pm 0.9$ & $6.7 \pm 1.3$ \\
5/7 Days & $6.9 \pm 0.6$ & $5.6 \pm 0.7$ & $6.3 \pm 0.7$ & $6.4 \pm 1.2$ \\
3 Months & $5.4 \pm 0.9$ & $6.0 \pm 0.5$ & $6.6 \pm 0.5$ & $7.1 \pm 1.9$ \\
6 Months & $6.1 \pm 1.5$ & $6.1 \pm 0.4$ & $6.4 \pm 0.7$ & $7.0 \pm 1.6$ \\
\hline
\end{tabular}




\section{Plateau phase fibrils are homogeneous}

On reaching the plateau phase (5/7 days), which is commonly interpreted as the end phase in fibril formation, in agreement with results of chapter 2 the morphology of all the samples (except A53T) became more homogeneous than at the early fibrillar stage (Figures 3.3 and 3.4: $2 / 3$ and 5/7 days). Wt $\alpha$ Syn at 7 days showed fibrils with average periodicity of $107 \pm 9 \mathrm{~nm}$ in contrast to a periodicity of $84 \pm 28 \mathrm{~nm}$ at 3 days. A30P fibrils showed a narrower distribution of periodicities with an average value of $105 \pm 7$ $\mathrm{nm}(118 \pm 23 \mathrm{~nm}$ at 2 days). The periodicity distribution of E46K at $88 \pm 19 \mathrm{~nm}$ was broader than those of wt and A30P aSyn, but was significantly narrower than the periodicity distribution of $\mathrm{E} 46 \mathrm{~K}$ at the earlier time point of 2 days $(124 \pm 80 \mathrm{~nm})$ with a prominent shift towards fibrils of shorter periodicities. A53T fibrils however showed two populations with approximate periodicities of 180 and $350 \mathrm{~nm}$ at 5 days $(282 \pm 87$ $\mathrm{nm}$ on average) in comparison to 2 days $(347 \pm 50 \mathrm{~nm})$ fibrils. The heights of the fibrils did not show any major changes and averaged between 5.6 and $6.9 \mathrm{~nm}$ among the fibrils (wt, A30P, E46K, A53T) from the plateau phase (Figure 3.6 and Table 3.3).

Our data on the morphology of the fibrils at the late lag phase and plateau phase of ThT assays show that fibril samples that are homogeneous at the plateau phase of the aggregation can show remarkable polymorphism in the lag phase. This is consistent with the hypothesis that the soluble unfolded protein molecules can adopt numerous aggregation prone conformations in solution of which no single conformation is energetically favored (Pellarin, Schuetz et al. 2010). Moreover, many of the numerous critical nuclei that are formed can form fibrils of distinct morphology and contribute to the frequently observed polymorphism (Heise, Hoyer et al. 2005; Paravastu, Leapman et al. 2008; Arosio, Knowles et al. 2015). However, in a particular solution condition one of the polymorphs could be kinetically favored either by higher rate of monomer addition or by pronounced secondary nucleation i.e. fragmentation or surface catalysis. As a result, the selected polymorph incorporates the major fraction of available monomers. Based on our data, the other polymorphs possibly disassemble over time, as fibrils with all but one mean periodicity disappear, and monomers reassemble into the dominant morphology observed in the plateau phase fibrils. Therefore, fibrils measured from plateau phase samples in wt and disease mutants, except for A53T, show a narrow fibril periodicity distribution. Notably all four sequences of aSyn form fibrils of distinct 
morphology in the same solution conditions (although wt and A30P are very similar) (Giasson, Uryu et al. 1999; van Raaij, Segers-Nolten et al. 2006; Heise, Celej et al. 2008; Lemkau, Comellas et al. 2012; Nielsen, Macchi et al. 2013). Given the considerable changes in the distribution of fibril periodicity during the active fibrillization, we decided to follow variations, if any, in the same fibril samples when stored for a long period.

\section{Wt fibrils mature over time}

The ThT plateau phase fibrils were stored at $4{ }^{\circ} \mathrm{C}$ without shaking. At intervals of 2 weeks, 1 month and 2 months a qualitative check of the morphology was done by AFM imaging. None of the samples showed major differences in the fibril morphology in comparison to the plateau phase. However, at 3 months the wt aSyn fibrils displayed distinct changes in morphology (Figure 3.2). Only $76 \%$ of the fibrils were periodic with a much wider periodicity distribution and a larger mean periodicity value of $309 \pm 60$ nm. $20 \%$ of the fibrils were designated as heterogeneous and $4 \%$ as non-periodic. Interestingly, most of these wt fibrils also manifested the wavy feature observed for A53T fibrils at 2 days. Thus, there were striking similarities between wt fibrils of 3 months and A53T fibrils of 2 and 7 days (Figures 3.2, 3.3, 3.4 and 3.5 and Table 3.1). Fibrils prepared from the A30P, E46K, and A53T variants however did not show any significant change in the mean periodicity of the fibrils over time (Figures 3.3 and 3.4 and Tables 3.1 and 3.2). The E46K sample contained $95 \%$ periodic fibrils, while the A53T had $\approx 55 \%$ periodic fibrils. The height of wt fibrils was on average $1.5 \mathrm{~nm}$ lower at 3 months than at the plateau phase. This is most likely due to the difference in the ratio of pixels representing the peak and trough heights in AFM images of fibrils of different periodicities (for details refer to chapter 1: atomic force microscopy). The mean fibril height of A30P and E46K fibrils did not change significantly in comparison to fibrils of plateau phase. However, the height of A53T fibrils showed a broader distribution (Figure 3.4).

Next, the fibrils were analyzed at 6 months following the same procedure. The wt fibrils showed a clear quantitative change in morphology with only $29 \%$ of the fibrils displaying a periodic twist with mean fibril periodicity of $315 \pm 83 \mathrm{~nm}$ (Tables 3.1 and 3.2). None of the disease mutants appeared to change in periodicities at 6 months (Figure 3.5). The heights of the wt and A53T fibrils exhibited a broad distribution with 
a small population showing approximately double heights (Figure 3.4). This increased height is speculated to be due to bundling or juxta-positioning of the fibrils. A qualitative check at 12 months did not show notable difference in the aSyn fibrils (Figure 3.7).
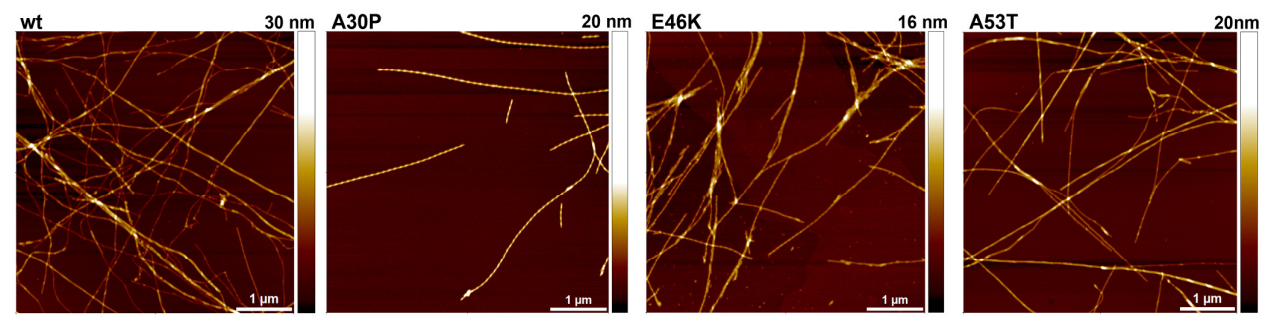

Figure 3.7 Representative AFM height images of wt, A30P, E46K and A53T fibrils at 1 year. Image size: $5 \times 5 \mu \mathrm{m}$.

The fibrils at the plateau phase in ThT assays are normally considered to be in equilibrium with the monomers and the intermediate aggregating species, and are therefore assumed not to undergo major transitions. However, quantitative measurements on single fibrils by AFM in samples up to 6 months evidently show that wt aSyn fibrils change while they are stored at $4{ }^{\circ} \mathrm{C}$. This is in agreement with recent studies showing that the plateau phase fibrils are in a quasi-equilibrium state (Vilar, Chou et al. 2008; Guilliams, El-Turk et al. 2013; Ma, Komatsu et al. 2013; Nystrom, Psonka-Antonczyk et al. 2013). In vivo studies on plaques of $A \beta$ in transgenic mouse model, using luminescent conjugated oligothiophene probes, have previously suggested conformational changes in $\mathrm{A} \beta$ fibrils with aging. From in vitro experiments these changes were proposed to arise due to maturation which is postulated be an intrinsic property of $A \beta$ fibrils and not because of an extrinsic influence (Nystrom, PsonkaAntonczyk et al. 2013). It is therefore plausible that wt aSyn fibrils also undergo a maturation process as seen in the present in vitro study. The disease mutants however, do not show such maturation. In view of the NMR evidence for perturbed structured regions in the mutants in comparison to wt (Comellas, Lemkau et al. 2011) it is likely that the mutants have distinct preferred conformations which lead to thermodynamically more stable fibrils (Sahay, Ghosh et al. 2015). 


\section{Dot blot on stored samples}

Wt aSyn fibrils thus exhibit a maturation reflected in changes in morphology over time while the fibrils of the disease mutants do not. To ascertain that this morphological change is not due to degradation of aSyn molecules in the fibrils while in storage, a dot blot was done on fibrils with antibodies against $\alpha$ Syn 15-123 and 121-125 residues. Both the antibodies showed identical reactivity to fibrils from the plateau phase and 1 year old fibrils (wt and disease mutants: matured fibrils) indicating that the protein molecules in the fibrils had intact epitopes. Therefore, we conclude that the protein monomers in the fibrils do not degrade while in storage at $4{ }^{\circ} \mathrm{C}$ for at least up to a year (Figure 3.8).

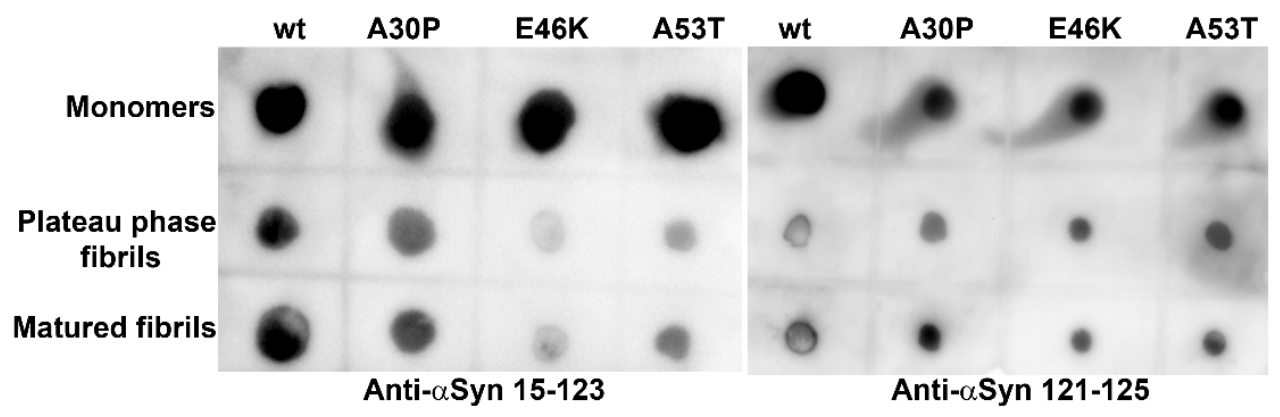

Figure 3.8 Dot blots of plateau phase and matured (1 year) aSyn fibrils using anti- $\alpha$ Syn 15-123 and anti- $\alpha$ Syn 121-125 antibodies. Both the antibodies show identical reactivity against plateau phase fibrils and matured fibrils.

\section{$\beta$-sheet content of plateau and matured fibrils}

To understand further the nature of the morphological differences in wt aSyn in terms of secondary structure, we performed attenuated total reflectance-Fourier transform infrared spectroscopy (ATR-FTIR) experiments on fibrils of wt and disease mutants at plateau phase and 1 year. Deconvolution of the ATR-FTIR spectra followed by curvefitting of the amide I peaks showed a $\beta$-sheet content of about $65-70 \%$ for all tested fibrils with a major peak located between 1627 and $1630 \mathrm{~cm}^{-1}$. The ATR-FTIR results showed no significant differences in the spectra of plateau phase and matured wt aSyn fibrils or the disease mutants, indicating similar $\beta$-sheet content even in fibrils with 
distinct morphology. The origin of the observed morphological changes in the fibrils is therefore likely due to tertiary and/or quaternary structural rearrangements in the fibrils with time (Figure 3.9 and Table 3.4).
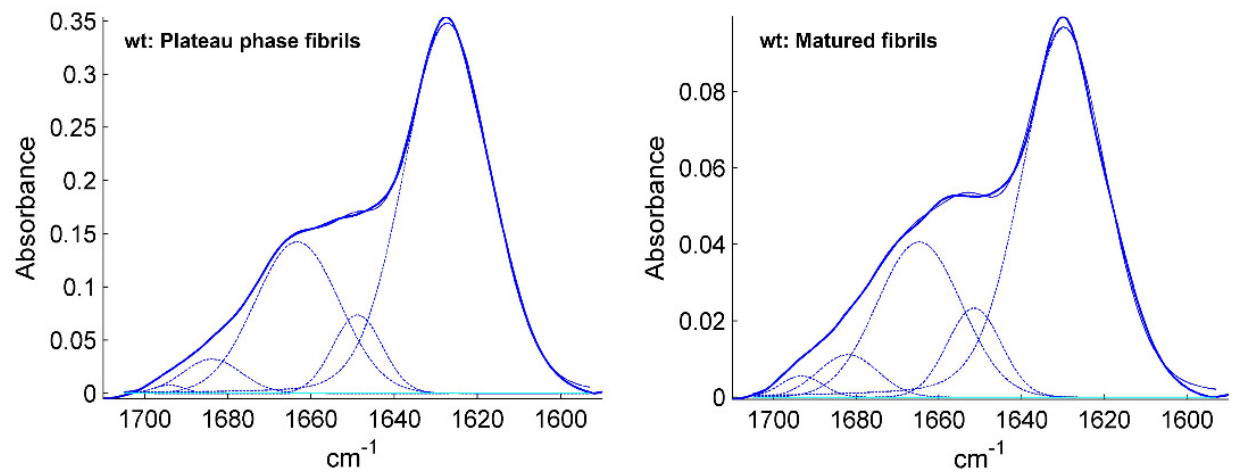

Figure 3.9 Curve-fitting in the amide I region of the ATR-FTIR spectra for plateau phase (left) and matured (1 year old: right) wt fibril samples.

Table 3.4 Summary of the $\beta$-sheet content of plateau phase and matured ( 1 year old) $\alpha$ Syn fibrils (wt and disease mutants).

\begin{tabular}{ccccc}
\hline $\boldsymbol{\beta}$-sheet content & Wt $\left(\mathbf{M a x ~ c m}^{-1}\right)$ & A30P $\left(\mathbf{M a x ~ c m}^{-1}\right)$ & E46K $\left(\mathbf{M a x ~ \mathbf { ~ m } ^ { - 1 } )}\right.$ & A53T $\left(\mathbf{M a x ~ c m} \mathbf{~ c}^{-1}\right)$ \\
\hline Plateau phase fibrils & $68 \%(1627)$ & $66 \%(1629)$ & $66 \%(1628)$ & $68 \%(1629)$ \\
Matured fibrils & $69 \%(1630)$ & $66 \%(1629)$ & $64 \%(1628)$ & $70 \%(1630)$ \\
\hline
\end{tabular}

Two distinct molecular mechanisms could account for the observed morphological changes in wt aSyn. In a competitive growth model, as reported for prion strains, the soluble monomers could adopt different conformations that result in fibrils of different morphologies. These conformations likely compete for the monomer fraction during the aggregation process and the kinetically favored conformation (faster monomer addition and/or more breakage) outcompetes the rest at plateau phase. However, once in storage at $4{ }^{\circ} \mathrm{C}$, a different fibril conformation is kinetically and thermodynamically favorable which accumulates over time. For this mechanism, it is imperative that fibril assembly be a reversible process and the fibrils formed at plateau phase fall apart and are recycled into fibrils of a different morphology at $4{ }^{\circ} \mathrm{C}$. In a maturation process, the 
fibrils formed at plateau phase do not fall apart in storage but undergo a slow transition at a single fibril level and mature over time leading to a thermodynamically more stable conformation.

The first mechanism is evident during the early fibrillization since at early fibrillar phase, fibrils of different morphologies are apparent and one of these morphologies becomes the major population at the plateau phase. However, it is difficult to establish the basis for the long-term transitions occurring at $4{ }^{\circ} \mathrm{C}$ in wt aSyn. Assuming there is competitive growth at $4{ }^{\circ} \mathrm{C}$, there are two possible scenarios. In the first instance, we assume that the critical monomer concentration (CMC) required for the formation of aggregating nucleus of $4{ }^{\circ} \mathrm{C}$ conformation is higher than the RMC at plateau phase at $37^{\circ} \mathrm{C}$. In this scenario, the existing fibrils should cold denature appreciably to provide sufficient monomers to form new fibrils (Bousset, Pieri et al. 2013; Ikenoue, Lee et al. 2014).

In the second scenario, we assume the $\mathrm{CMC}$ for the $4{ }^{\circ} \mathrm{C}$ conformation to be much lower than the RMC at plateau phase. In this case, the amount of monomers in the supernatant at plateau phase is enough for nucleation and fibril growth from the slow dissociation of plateau phase fibrils at $4{ }^{\circ} \mathrm{C}$. If the second scenario holds, an aggregation at $4{ }^{\circ} \mathrm{C}$ with much lower concentration than $100 \mu \mathrm{M}$ wt $\alpha$ Syn should form fibrils with morphologies characteristic of stored fibrils. To understand further the nature of morphological change in wt aSyn, we studied the possibility of both the scenarios.

\section{Stability of plateau phase fibrils}

Some recent reports on aSyn have shown that fibrils prepared at $37^{\circ} \mathrm{C}$ can disassemble into monomers within hours when kept at lower temperatures (Bousset, Pieri et al. 2013; Ikenoue, Lee et al. 2014). This reversible nature of the fibrils was however shown to be dependent on the solution conditions used for fibrillization (Bousset, Pieri et al. 2013). We systematically tested for this possibility. Aggregation reactions (wt and mutants) at plateau phase were centrifuged and the RMC was determined, then new buffer was added to the fibril pellet to restore the original volume and the fibrils were incubated for a week at $37^{\circ} \mathrm{C}$ without shaking to prevent fibril breakage. This procedure of centrifugation and RMC determination was done over 3 weeks collecting three time points. Negligible amounts of monomers were recovered in the second and subsequent RMC analyses. Next, the same procedure was repeated but the fibrils were stored at 4 
${ }^{\circ} \mathrm{C}$; this too did not show significant monomer recovery in the supernatant fractions. In addition, ThT analyses from the same samples showed consistent presence of ThT positive $\beta$-sheet structures (Figure 3.10). Thus, the fibrils do not dissociate at lower temperature of $4{ }^{\circ} \mathrm{C}$ and the overall equilibrium is not shifted towards disassembly under the conditions used in our experiments.

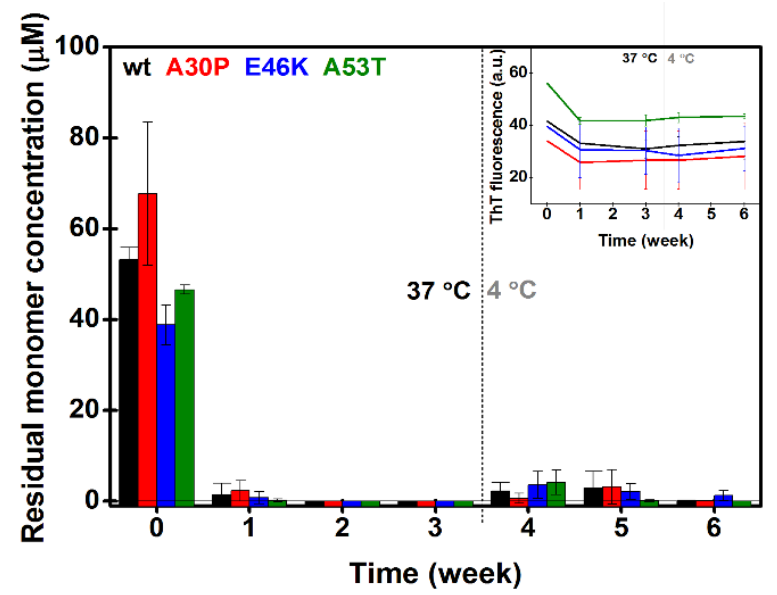

Figure 3.10 Plot showing irreversible nature of wt and mutant aSyn fibrils prepared in $10 \mathrm{mM}$ Tris$\mathrm{HCl}, 10 \mathrm{mM} \mathrm{NaCl}$ and $0.1 \mathrm{mM}$ EDTA at $\mathrm{pH}$ 7.4. RMC shows that after removal of monomers from a plateau phase reaction (ThT assay) at week zero, the monomer fraction in the supernatant is not replenished in fibrils stored at 37 or $4{ }^{\circ} \mathrm{C}$. Inset: A parallel ThT assay every alternate week showed the presence of $\beta$-sheet positive fibrils in reactions over the same period.

\section{Fibrillization at low temperature}

To examine if the wt fibril morphology at $4{ }^{\circ} \mathrm{C}$ (i.e. periodicity of about $300 \mathrm{~nm}$ with heterogeneous and non-periodic fibrils) is a result of a kinetically preferred conformation at low temperature, we aggregated wt aSyn at $4{ }^{\circ} \mathrm{C}$. Aliquots were withdrawn at an interval of two weeks for ThT assay and AFM samples. By the end of 1 month, few short fibrils could be seen in AFM images but no increase in ThT fluorescence intensity was detected (data not shown). After 3 months, long fibrils similar to fibrils produced at $37^{\circ} \mathrm{C}$ with a periodicity of about $100 \mathrm{~nm}$ were observed. Thus even at low temperature periodic fibrils appear first. The same fibril sample imaged after 1 year showed heterogeneous and non-periodic fibrils (Figure 3.11). This 


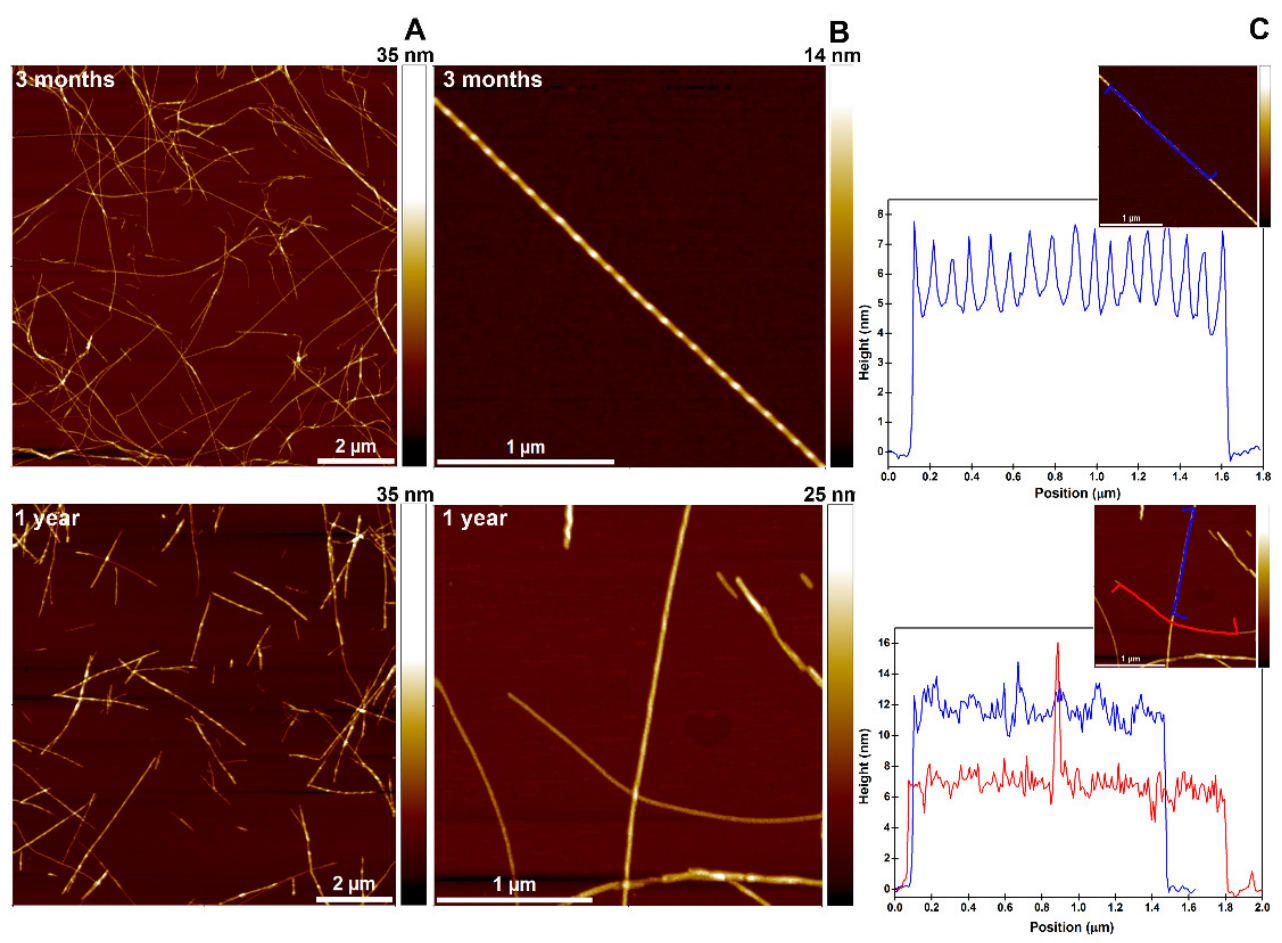

Figure 3.11 Representative AFM height images of wt fibrils showing periodic fibrils grown at $4{ }^{\circ} \mathrm{C}$ with shaking after 3 months of incubation. After 3 months, the measured fibril periodicity was about $100 \mathrm{~nm}$ (upper panel). Same fibril sample imaged after 1 year shows fibrils with heterogeneous and non-periodic morphology (lower panel). (A) Overview images. (B) Detailed images. (C) Longitudinal height profile of the fibrils as indicated in the inset.

suggests that the transitions observed in the fibrillar morphologies is independent of temperature and is most likely an inherent characteristic of the wt aSyn fibrils.

Thus, the morphology of the wt fibrils is governed by two mechanisms. In the aggregation reaction, initially a prion strain-like competitive growth determines the morphology of the plateau phase fibrils. Thereafter a slow maturation process takes over, possibly involving rearrangements in the tertiary and/or quaternary structure of the fibrils. To comment on the precise nature of the rearrangements (if any) between the plateau phase fibrils and the mature fibrils, additional in-depth structural study is required. Further, it is reasonable to assume that the morphological transitions seen over months in wt aSyn could happen at a shorter time scale at $37^{\circ} \mathrm{C}$. We also note that the results presented were obtained at low salt conditions and it is difficult to predict 
whether similar behavior can be expected from $\alpha$ Syn fibrils in vivo, where the solution and molecular milieu is very different. Nonetheless, the present study illustrates the importance of mechanisms like competitive growth in determining morphological characteristics of fibrils assembled from different aSyn sequences and shows that while wt $\alpha$ Syn exhibits the process of maturation, the disease mutants form morphologically different and more stable fibrils at the solution conditions used.

\section{CONCLUSIONS}

Together our results show that during the early stages of fibrillization several distinct morphologies for all the aSyn sequences exist, providing empirical evidence that the same sequence of the protein can attain a number of conformations that form fibrils of different morphologies. This ability to sample various amyloid conformations appears to be an intrinsic property of the polypeptide sequence and bears a resemblance to the strain-like behavior of prions. In complement to the recent reports on prion-like hypotheses for amyloids, based on cell-to-cell transmission of pathogenesis (George, Rey et al. 2013; Jucker and Walker 2013; Watts, Giles et al. 2013; Goedert, Falcon et al. 2014), our study highlights the property of competitive growth between polymorphs of the misfolded aSyn. Further observation of evolution of wt fibril morphology over time, in contrast to the stability of the mutant fibrils' morphology, underscores fundamental differences in the way protein molecules with one amino acid difference assemble into fibrils in the same solution conditions. Since the fibrils do not significantly disassemble or degrade when stored, it is likely that the wt fibrils undergo a process of maturation. In this maturation the individual fibrils possibly go through some rearrangement leading to formation of thermodynamically favorable state(s), which manifests as heterogeneous and non-periodic fibrils over time.

\section{ACKNOWLEDGEMENTS}

We thank Kirsten van Leijenhorst-Groener, Yvonne Kraan and Nathalie Schilderink for protein expression and purification. Dr. Martin Bennink, Kees van der Werf and Robert Molenaar for advice on AFM and Dr. Vincent Raussens for help with ATR-FTIR experiments, data analyses and interpretation. 


\section{Chapter 4}

\section{Thioflavin-T fluorescence intensity depends on subtle differences in $\alpha$-synuclein fibril morphology}

In recent years, the observed polymorphic nature of amyloid fibrils, and probably associated prion-like properties, have resulted in an increased interest in polymorphspecific characteristics of amyloids. Thioflavin-T (ThT) is the most commonly used fluorescent probe for following in vitro amyloid formation. Despite its widespread use the binding of ThT to amyloid fibrils is not fully understood. In the present chapter, we study the morphological templating and ThT fluorescence intensity of two aSyn fibril species with noticeably different morphologies. We find that both polymorphs template their morphologies over 2 generations of seeded aggregations. Efficient morphological templating enabled us to delineate the effects of primary sequence and secondary/tertiary structure of the fibrils on fibril-ThT interactions. Fibrils formed of the same sequence of protein but with different morphologies appear to have two qualitatively distinct binding sites for ThT on the fibril surface that results in typespecific fluorescence behavior in ThT assays.

Parts of this chapter will be published as: Arshdeep Sidhu, Francesco Simone Ruggeri, Giovanni Dietler, Christian Blum, Ine Segers-Nolten, Vinod Subramaniam. Thioflavin-T fluorescence intensity depends on subtle differences in $\alpha$-synuclein amyloid fibril morphology. 


\section{INTRODUCTION}

Amyloid formation in in vitro experiments is usually probed by Thioflavin-T (ThT) based fluorescence assays (Groenning 2010). ThT fluorescence shows an amyloidspecific enhancement of fluorescence intensity on binding amyloid fibrils, and exhibits greater sensitivity and somewhat better reproducibility in comparison to other common dye-based methods of amyloid detection, like Congo red and Thioflavin-S assays (Conway, Lee et al. 2000; Groenning 2010). ThT assays are not reliable for absolute quantitative experiments, but in the absence of better alternatives, nevertheless remain a standard choice for comparative aggregation studies investigating mechanistic details of amyloid formation (Conway, Harper et al. 1998; Conway, Lee et al. 2000; Giehm, Lorenzen et al. 2011; Cohen, Linse et al. 2013; Ghosh, Mondal et al. 2013; Buell, Galvagnion et al. 2014; Ghosh, Sahay et al. 2014). Despite the prevalent use of ThT for amyloid studies, observations like the spread in ThT fluorescence intensities between repeats in any given aggregation experiment, and the detailed mechanisms and factors influencing amyloid fibril-ThT interactions are not well understood.

ThT binding: ThT is a cationic benzothiazole rotor molecule with a high degree of rotational freedom over the carbon-carbon bond linking the benzylamine and benzathiole ring (Figure 4.1A). In solution, the ThT molecule is free to rotate around the carbon-carbon bond leading to quenched fluorescence. On binding to amyloid fibrils, the rotational freedom about the carbon-carbon bond is lost, resulting in a chiral twisted conformation, which impedes non-radiative decay and thus enhancing the fluorescence (Biancalana and Koide 2010). The ThT fluorescence thus gives an easy read out for protein fibrillization. The molecular mechanism of the ThT-amyloid interaction is however complex. ThT is suggested to bind on the fibril surface, between the binding channels formed by the amino acid sidechains, oriented parallel to the long axis of the fibrils (Krebs, Bromley et al. 2005; Biancalana and Koide 2010). In the interaction with $\beta_{2}$-microglobulin oligomers however, the ThT orientation is proposed to be perpendicular to the $\beta$-strands (Wolfe, Calabrese et al. 2010). ThT is reported to have multiple binding sites on a fibril with comparable binding affinities (LeVine 1999). Studies on $A \beta$ fibrils suggested the presence of three high affinity binding sites (BS1, 2 and 3) with different binding stoichiometries for ThT: protein monomers (1:35, 1:4 and 1:300, respectively) (Lockhart, Ye et al. 2005). Further, simulation studies on amyloid 
fibril-ThT interactions indicate a preference for spatially consecutive aromatic (tyrosine and phenylalanine) and hydrophobic (valine and leucine) amino acids in the binding channels as opposed to charged residues, thus underscoring a sequence-based predisposition (Wu, Wang et al. 2007; Wu, Biancalana et al. 2009).

Fibril morphology and ThT binding: Single amino acid differences in amyloidogenic proteins can influence the aggregation behavior, structure and the morphology of the fibrils produced (Conway, Harper et al. 1998; van Raaij, SegersNolten et al. 2006; Heise, Celej et al. 2008). Distinct fibril morphologies, as observed by AFM and EM (Qiang, Kelley et al. 2013), of A $\beta$ were reported to arise from variations in the structural conformations of the $\beta$-sheet rich monomers in the fibrils (Paravastu, Leapman et al. 2008). These conformational differences are likely to result in altered boundaries of the $\beta$-strand segments and in different quaternary interactions between the protofibrils (illustrated for two aSyn polymorphs in Figure 4.1B, C and D) (Paravastu, Leapman et al. 2008; Gath, Bousset et al. 2014). Thus, fibrils with distinct morphologies can be expected to have characteristically different surface features, which could influence the interactions with amyloid-binding compounds (Nielsen, Macchi et al. 2013; Lindberg, Wranne et al. 2015).
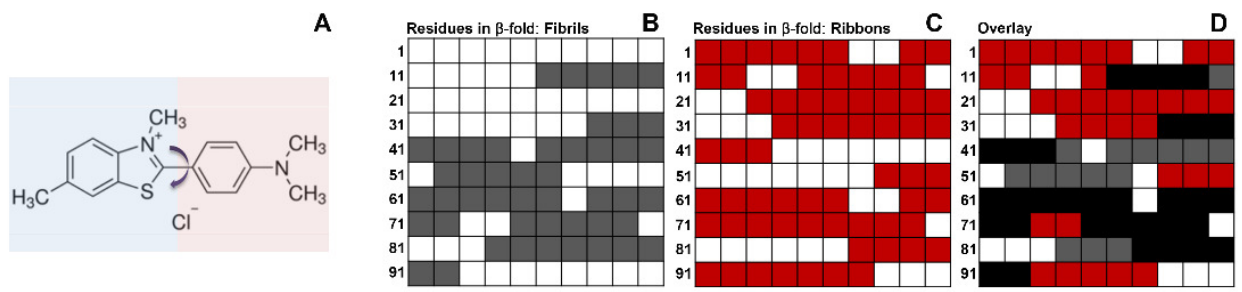

Figure 4.1 (A) Structure of ThT molecule with benzylamine group (shaded in pink) and benzathiole ring (shaded in blue) bonded by a rotatable carbon-carbon bond. (B and C) Residues 1-100 of $\alpha$ Syn in $\beta$-fold in two polymorphs of $\alpha$ Syn, fibrils in grey boxes and ribbons in red boxes. (D) An overlay of B and $\mathrm{C}$ shows that few residues (in black) share the $\beta$-fold motif in these two polymorphs (based on information from Gath, Bousset et al. 2014).

In chapter 3, we observed that $\mathrm{A} 30 \mathrm{P}$ and $\mathrm{A} 53 \mathrm{~T}$ aSyn monomers fibrillize into morphologically distinct fibrils. Following the same aggregation conditions, in this chapter, we study the characteristic features of the two morphologies (A30P and A53T fibrils). We probe morphological templating by A30P and A53T fibrils, used as seeds, 
on wt $a$ Syn monomers over 2 generations in the seeded aggregations. In the process, we produce chemically identical fibrils with distinct morphologies. The fibrils thus produced are analyzed for their ThT binding features with respect to the ThT fluorescence intensity and the fluorescence lifetimes of the bound ThT. We find that fibrils with different morphologies present qualitatively different binding sites for the ThT molecules, and that the fluorescence intensity is dependent on the ThT concentration.

\section{MATERIALS AND METHODS}

\section{$\alpha$ Syn purification and fibrillization}

Wt and two disease associated mutants of aSyn (A30P and A53T) were expressed and purified as described earlier (Sidhu, Segers-Nolten et al. 2014). A30P and A53T were fibrillized in a de novo aggregation as follows: $250 \mu \mathrm{M}$ monomeric stocks, frozen at -80 ${ }^{\circ} \mathrm{C}$ were thawed and prepared at $100 \mu \mathrm{M} \alpha$ Syn in $10 \mathrm{mM}$ Tris- $\mathrm{HCl}, 10 \mathrm{mM} \mathrm{NaCl}, 0.1$ mM EDTA and $20 \mu \mathrm{M}$ ThT, at $\mathrm{pH} 7.4$ ( $\mathrm{F}_{0}$ generation). All the reactions were prepared in triplicates in $200 \mu \mathrm{l}$ volume and incubated in 96 well plates with optical bottoms (Nunc, Thermo Fisher Scientific), sealed with adhesive film (Viewseal, Greiner Bio One). The plates were incubated at $37^{\circ} \mathrm{C}$ with orbital shaking in a Safire ${ }^{2}$ microplate reader (Tecan) for 96 hours. The aggregations were monitored by using $446 \mathrm{~nm}$ excitation and following the ThT fluorescence emission intensity (bottom reading) at $485 \mathrm{~nm}$. Readings were taken every 15 minutes.

\section{Atomic force microscopy (AFM)}

AFM samples were prepared at the plateau phase of the ThT assay to compare the morphology of the fibrils. The samples were prepared by about 10 -fold dilution of the aggregation reactions in aggregation buffer. The samples $(10 \mu \mathrm{l})$ were adsorbed on freshly cleaved mica (Muscovite, V-1 quality, EMS) for 4 minutes, followed by 2 gentle washes with $100 \mu \mathrm{l}$ Milli-Q water and drying in a mild stream of nitrogen gas, filtered through a $0.22 \mu \mathrm{m}$ filter. AFM images were acquired on a Bioscope Catalyst instrument (Bruker) in soft tapping mode in air using a NSC36 probe, tip B, with a force constant of $1.75 \mathrm{~N} / \mathrm{m}$ (NanoAndMore). All images were captured at $512 \times 512$ pixels per image at $0.5 \mathrm{~Hz}$ scan rate. Images were further processed using Scanning Probe Image Processor (SPIP)-6.0.13 software (Image Metrology). 


\section{Residual monomer concentration (RMC) determination}

To determine the amount of soluble monomers in the aggregation reaction at the plateau phase (as determined by the ThT assay), $100 \mu$ of the solution containing the aggregated protein was centrifuged at $21,000 \times \mathrm{g}$ at room temperature for 1 hour in an IEC Micromax microcentrifuge (Thermo Fisher Scientific). $50 \mu \mathrm{l}$ of the supernatant was removed and the absorbances at 280 and $330 \mathrm{~nm}$ respectively, were measured on a NanoDrop ND-1000 spectrophotometer (Isogen Life Science). The absorption at 280 $\mathrm{nm}\left(\mathrm{A}_{280}\right)$ was corrected for scattering contributions $\left(\mathrm{A}_{330}\right)$, possibly from oligomeric assemblies, before calculation of the RMC (Grimsley 2004) using an extinction coefficient of $5600 \mathrm{M}^{-1}$. $\mathrm{cm}^{-1}$. The residual concentration of ThT was calculated by measuring the absorbance of the supernatant at $412 \mathrm{~nm}$ and using an extinction coefficient of $26,620 \mathrm{M}^{-1} \cdot \mathrm{cm}^{-1}$.

\section{Seeded aggregations}

Seeded aggregations were performed using $2 \mu \mathrm{M}$ pre-formed fibrils as aggregation nuclei (seeds). Seeds were prepared by sonicating $100 \mu \mathrm{l}$ of A30P and A53T fibrils (from $\mathrm{F}_{0}$ ) in a bath sonicator (Branson 1510) for 2 minutes in thin walled $200 \mu \mathrm{l}$ PCR tubes. Aggregation reactions were set up as mentioned above but with $98 \mu \mathrm{M}$ wt $\alpha$ Syn monomers and $2 \mu \mathrm{M}$ seeds (based on initial monomer concentration) of A30P and A53T fibrils (from $\mathrm{F}_{0}$ ). Fibrillization was followed as previously stated (resultant fibrils: $\left.F_{1}\right)$. Next, the fibrils formed in the $F_{1}$ generation were used as seeds $(1 \mu \mathrm{M})$ following the same protocol as above to produce $\mathrm{F}_{2}$ generation fibrils. The fibrils produced in the $\mathrm{F}_{1}$ and $\mathrm{F}_{2}$ generations are called $\mathrm{A} 30 \mathrm{P}$ and $\mathrm{A} 53 \mathrm{~T}$ templated fibrils based on the seeds used from the $\mathrm{F}_{0}$ aggregation.

\section{ThT fluorescence lifetime measurements}

The fluorescence lifetimes of ThT when bound to A30P and A53T templated fibrils $\left(\mathrm{F}_{2}\right)$ were determined by analysis of the fluorescence lifetime decay curves obtained by time correlated single photon counting (TCSPC). TCSPC measurements were done on a single photon-counting controller FluoroHub connected to a Fluoromax-4 spectrofluorometer (HORIBA Jobin Yvon). The instrument response function (prompt) was measured by illumination of a Ludox AS-30 colloidal silica solution at $460 \mathrm{~nm}$. A pulsed diode light source, NanoLED-460 nm with a pulse duration of $1.3 \mathrm{~ns}$ 
and a repetition rate of $1 \mathrm{MHz}$ was used for illumination (slit width: $5 \mathrm{~nm}$ ). Next, the samples were illuminated with the same source and the emission was followed at 485 $\mathrm{nm}$ in a $5 \mathrm{~mm}$ path length quartz cuvette. The decay curves were analyzed using DAS6 software (HORIBA Jobin Yvon), using a 2-component exponential fit to obtain the lifetimes of the fast and slow components along with their relative amplitudes.

\section{Induced circular dichroism (ICD)}

CD spectra of ThT bound to fibrils were acquired for $100 \mu \mathrm{M} w \mathrm{w}, \mathrm{A} 30 \mathrm{P}$ and A53T fibrils in the presence of $50 \mu \mathrm{M}$ ThT solution in a $1 \mathrm{~mm}$ (100 $\mu$ fibril sample) and $5 \mathrm{~mm}(20$ $\mu \mathrm{l}$ fibril sample) path length cuvette on a JASCO J-1500 Circular Dichroism spectrometer. The spectra were acquired in continuous scanning mode with a data pitch of $1 \mathrm{~nm}$ between 350 to $500 \mathrm{~nm}$.

\section{RESULTS AND DISCUSSION}

\section{Fibrillization of A30P and A53T fibrils}

A30P and A53T monomers were both fibrillized in uniform aggregation conditions. In the aggregation reaction, which is expected to mirror the increase in amyloid content with an increase in ThT fluorescence intensity, A30P fibrils exhibited a higher final fluorescence intensity in comparison to A53T fibrils at the plateau phase (Figure 4.2A).
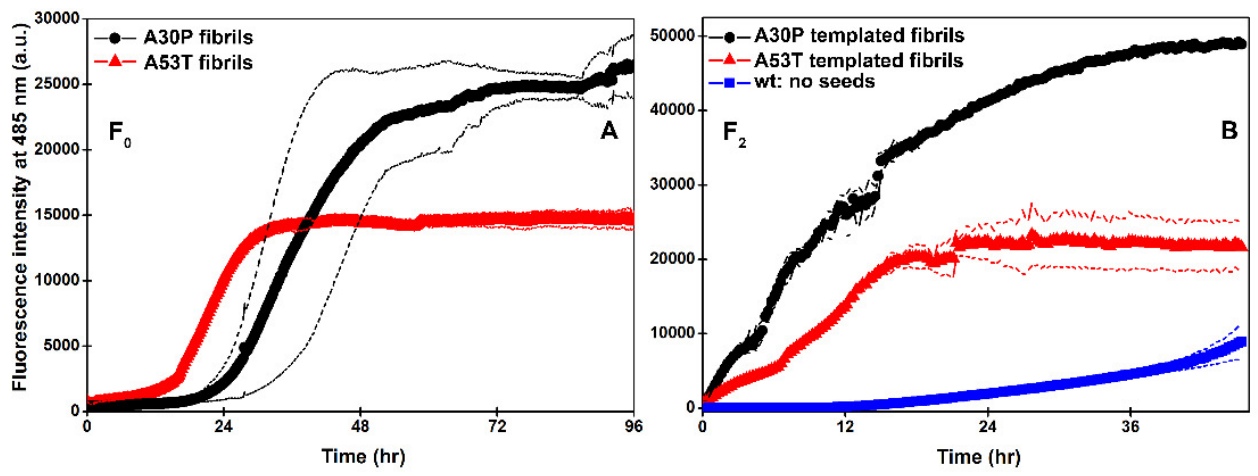

Figure 4.2 ThT aggregation kinetics for $(\mathrm{A})$ unseeded $\mathrm{A} 30 \mathrm{P}$ and $\mathrm{A} 53 \mathrm{~T}$ monomers ( $\mathrm{F}_{0}$ generation) and (B) seeded aggregation with wt monomers and A30P and A53T templating seeds ( $\mathrm{F}_{2}$ generation). Dotted lines around the curves denote standard deviation among the triplicates. 
The plateau phase samples typically contain a mix of fibrils, oligomers and monomers. However, separation of fibrils by high-speed centrifugation and correction of the $A_{280} \mathrm{~nm}$ signal for scattering (potentially a contribution from putative oligomers) enables assessment of the amount of soluble monomers in the supernatant. Unexpectedly, the thus measured residual monomer concentration (RMC) indicated a greater conversion of soluble monomers into fibrils in A53T aggregations as opposed to A30P aggregations (Figure 4.3: $\mathrm{F}_{0}$ ). The RMC determination shows that more fibrils are formed in the A53T aggregations; however, the ThT fluorescence does not mirror this difference. ThT apparently does not fluoresce as intensely when bound to A53T fibrils as when bound to a lower fibrillar mass of A30P fibrils. The observed differences in fluorescence intensities are suggestive of aSyn fibril type-specific ThT interactions. Results from chapter 3 show that the morphology of A30P and A53T fibrils is distinct in the aggregation conditions used here. To confirm the morphology of the A30P and A53T fibrils, we assessed the detailed morphology of the A30P and A53T fibrils prepared in this study by AFM.

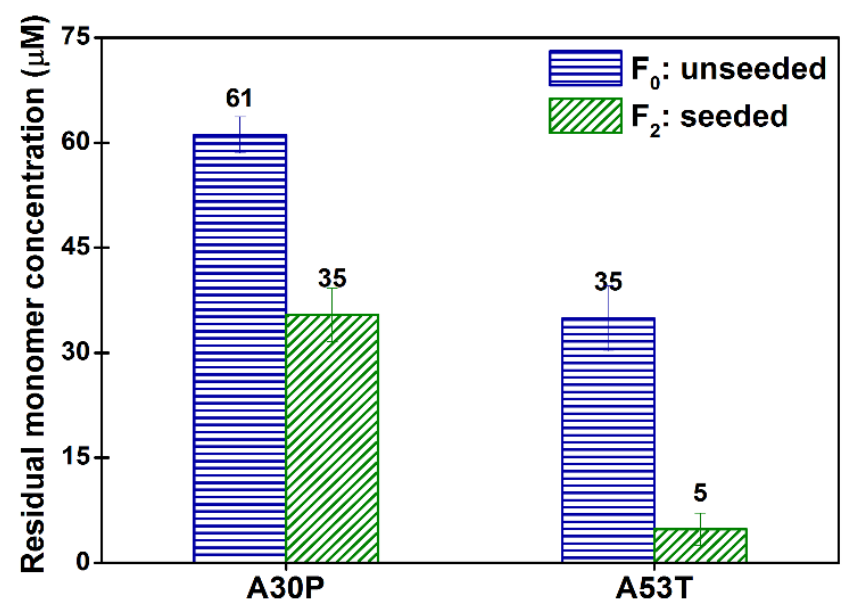

Figure 4.3 Residual monomer concentration of unseeded and seeded aggregation.

The fibrils from the plateau phase $\left(\mathrm{F}_{0}\right.$ generation), were imaged by tapping mode AFM to study the morphology of the fibrils. Both the samples contained several micron long fibrils (Figure 4.4A, B: inset). AFM height images revealed, in agreement with the results of chapter 3, that A30P fibrils had a periodic twist with an average periodicity of 
about $100 \mathrm{~nm}$ and height of about $6 \mathrm{~nm}$ (Figures 4.4A, $\mathrm{C}$ and 4.5A: upper panel). A53T fibrils, however, had a longer periodicity of about $350 \mathrm{~nm}$, a height of about $6 \mathrm{~nm}$ and a number of fibrils were observed to stick to each other (heterogeneous fibrils) (Figures 4.4B, C and 4.5A: lower panel). Thus, the disease mutants, with a single amino acid difference in the polypeptide sequence, formed fibrils of different morphology in the aggregation conditions used here. The inverse relation between ThT intensity and fibrillar mass, together with the observed morphological differences suggests that ThT may indeed experience different binding surfaces on morphologically distinct fibrils, thereby affecting its fluorescence intensity. However, the observed results could also be a result of differences in the primary sequence (chemistry) of the two disease mutants.
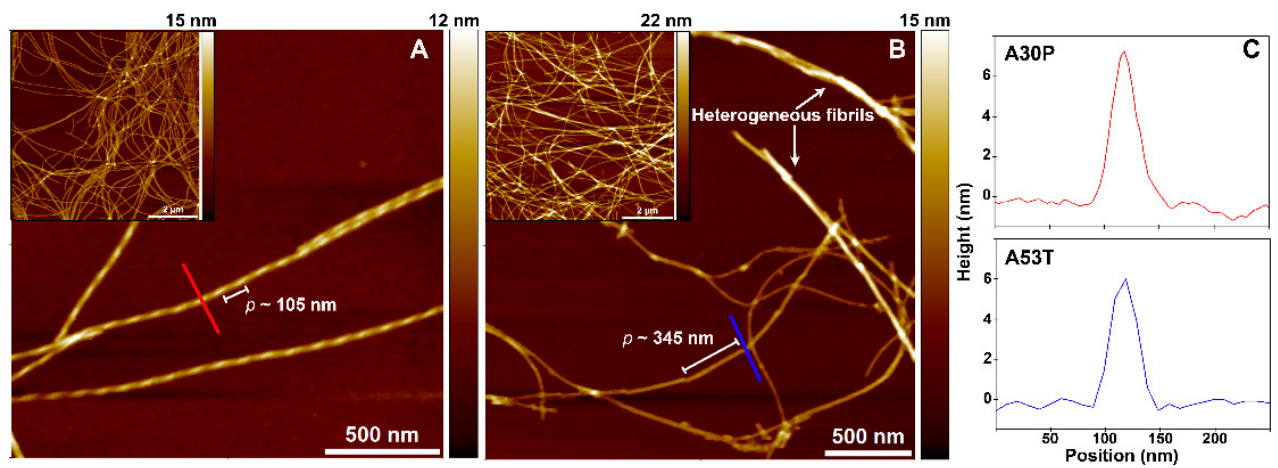

Figure 4.4 AFM height images of A30P (A) and A53T (B) fibrils $\left(\mathrm{F}_{0}\right)$ at plateau phase. Inset: overview images showing dense and long fibrils in both samples. White labels $(p)$ show the length of periodic twist in the respective fibrils. (C) Height cross-section of fibrils, A30P and A53T, at positions marked by red and blue lines in images (A and $\mathrm{B})$.

To delineate the effect of chemistry and/or morphology on the ThT fluorescence intensity we seeded the aggregation of wt protein with A30P or A53T fibril seeds. In the event of efficient seeding, the morphology of the A30P and A53T seeds would be recapitulated by the wt monomers resulting in chemically identical but morphologically distinct fibrils. Comparative aggregation of the produced fibrils can allow to delineate the significance of the fold and the primary sequence in the observed results. 


\section{Seeded aggregations}

Proteins with differences in the amino acid sequences are reported to form fibrils of different morphologies (van Raaij, Segers-Nolten et al. 2006). To investigate the role of fibril morphology on the ThT fluorescence we prepared chemically identical but morphologically different fibrils by seeding wt monomers with A30P or A53T seeds. Aggregations were performed for 2 generations with $2 \mu \mathrm{M}$ ( $\mathrm{F}_{0}$ fibrils) and $1 \mu \mathrm{M}\left(\mathrm{F}_{1}\right.$ fibrils) seeds, respectively, to ensure that the concentrations of A30P and A53T seeds in the final aggregation $\left(\mathrm{F}_{2}\right)$ are negligible (about $0.02 \mu \mathrm{M}$ ). The seeds were prepared by sonication of fibrils obtained in the $\mathrm{F}_{0}$ generation. Aggregation was monitored by changes in ThT fluorescence intensity and the fibrils from the plateau phase $\left(F_{1}\right.$ generation) were imaged by AFM to examine morphological templating. The transmission of seed morphology to wt aSyn monomers in a seeded aggregation has been previously reported (Bousset, Pieri et al. 2013). A30P seeded aggregations indeed produced fibrils with $\mathrm{A} 30 \mathrm{P}$ fibril morphology, while $\mathrm{A} 53 \mathrm{~T}$ seeded aggregations resulted in fibrils of A53T fibril morphology (Figure 4.5B). The fibrils from A30P and A53T seeded aggregations will be subsequently referred to as A30P and A53T templated fibrils, respectively. The next round of seeded aggregation $\left(\mathrm{F}_{2}\right)$ was performed with wt aSyn monomers, using A30P and A53T templated fibrils from the $\mathrm{F}_{1}$ generation as seeds. The fibrils produced ( $\mathrm{F}_{2}$ generation) recapitulated the fibril morphologies of the seeds observed in the $\mathrm{F}_{1}$ generation (Figure 4.5C). Therefore, at the plateau phase of the $\mathrm{F}_{2}$ generation both types of fibrils ( $\mathrm{A} 30 \mathrm{P}$ and $\mathrm{A} 53 \mathrm{~T}$ templated) are composed of the same monomers (wt aSyn) but exhibit the morphologies characteristic of A30P and A53T fibrils (Figure 4.5).

\section{ThT aggregation kinetics and RMC in seeded aggregations}

As expected for seeded aggregations, the fibrillization kinetics of $\mathrm{A} 30 \mathrm{P}$ and $\mathrm{A} 53 \mathrm{~T}$ templated fibrils, based on the ThT intensity assay, did not show a lag phase. A30P templated fibrils showed higher fluorescence intensity compared to the A53T templated fibrils from the start, and akin to the $\mathrm{F}_{0}$ generation had higher final fluorescence intensity (Figure 4.2B). The RMC however revealed incorporation of $95 \%$ of the monomers into fibrils in A53T templated fibrils as opposed to only $65 \%$ in A30P templated fibrils (Figure 4.3: $\mathrm{F}_{2}$ ), which showed more intense ThT emission. The lower fluorescence intensity of the A53T templated fibrils is not due to less ThT binding as 
calculations based on residual ThT concentrations showed that all ThT was bound in the A53T templated fibrils (Table 4.1). Therefore, compared to A53T templated fibrils, the lower mass of A30P templated fibrils results in a decidedly higher ThT fluorescence intensity with a comparable fraction of bound ThT. Observation of the same trend, in both the ThT intensity assay and RMC, between the non-seeded $\left(\mathrm{F}_{0}\right)$ and seeded $\left(\mathrm{F}_{2}\right)$ fibrils, must mean that the ThT fluorescence intensity is influenced by the fibril morphology.
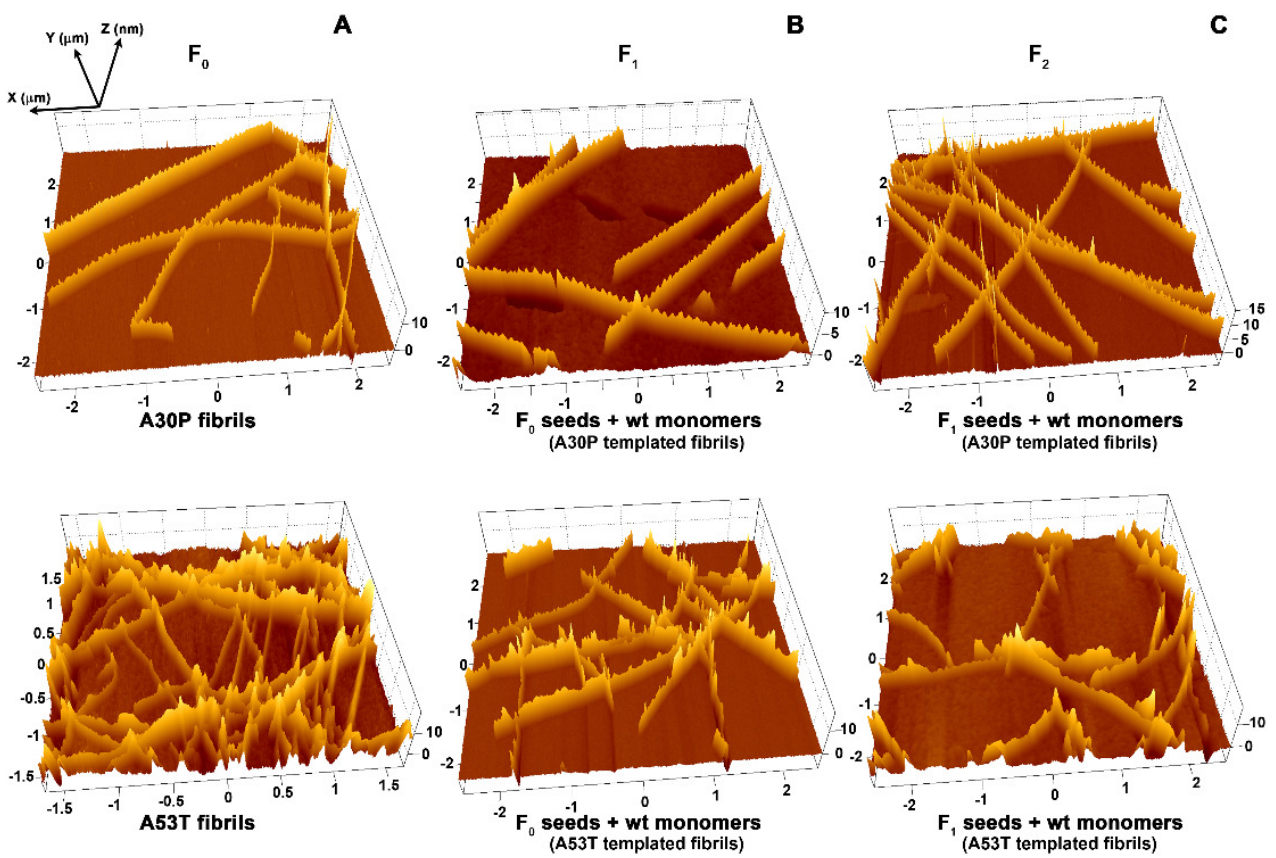

Figure 4.5 Representative AFM height images showing morphological templating by seeds in seeded aggregation reactions over two generations. (A) Fibrils from $F_{0}$ generation. (B) Fibrils of $F_{1}$ generation produced by using seeds from $\mathrm{F}_{0}$. (C) Fibrils of $\mathrm{F}_{2}$ generation produced by using seeds from $\mathrm{F}_{1}$.

Table 4.1 ThT to protein binding ratios for $\mathrm{F}_{2}$ generation fibrils ( \pm stdv among triplicates).

\begin{tabular}{lcc}
\hline & A30P templated fibrils & A53T templated fibrils \\
\hline Monomers in fibrils & $65 \pm 3.8 \mu \mathrm{M}$ & $95 \pm 2.3 \mu \mathrm{M}$ \\
ThT bound & $17 \mu \mathrm{M}$ & $20 \mu \mathrm{M}$ \\
$\mu \mathrm{M}$ ThT/ $\mu$ M monomer & 0.26 & 0.21 \\
\hline
\end{tabular}




\section{ThT fluorescence lifetime and relative amplitude}

The observed disparity in the ThT assay and RMC could be due to qualitatively different binding and hence fluorescence enhancement of ThT molecules in morphologically distinct fibrils. The simplest explanation could be that the fluorescence quantum efficiency of ThT bound to A30P templated fibrils is higher than that of ThT bound to A53T templated fibrils, which results in higher fluorescence. Higher fluorescence quantum efficiency of ThT when bound to A30P templated fibrils should give longer ThT fluorescence lifetimes. To probe this hypothesis, we measured the fluorescence lifetimes of ThT in A30P and A53T templated fibrils $\left(\mathrm{F}_{2}\right)$ by time correlated single photon counting (TCSPC). The fluorescence decay curves were analyzed with a 2component exponential fit, which was found sufficient to fit the data accurately. For control, the lifetime of $20 \mu \mathrm{M}$ ThT was measured in the absence of fibrils. The fast and slow component showed a lifetime of 6.98 ps ( $81 \%$ relative amplitude) and $0.98 \mathrm{~ns}$ (19 $\%$ relative amplitude), respectively.

In the presence of fibrils, the two components, with fast and slow lifetimes, most likely represent two qualitatively different binding sites on the fibrils, one resulting in a ThT population with a shorter lifetime $\left(\tau_{1}\right)$ and the other with a longer lifetime $\left(\tau_{2}\right)$. The fluorescence lifetime of the slow component $\left(\tau_{2}\right)$ when bound to A30P and A53T templated fibrils is not significantly different with values of $1.68 \pm 0.02$ and $1.64 \pm 0.04$ $\mathrm{ns}$, respectively. The fluorescence lifetime of the fast component $\left(\tau_{1}\right)$ of A30P templated fibrils and for A53T templated fibrils also agree well $(0.31 \pm 0.04$ and $0.22 \pm 0.01 \mathrm{~ns}$, respectively). The presence of 2 binding sites for ThT parallels the proposed multiple ThT binding sites with different binding affinities on the fibril surface (LeVine 1999; Lockhart, Ye et al. 2005). Furthermore all of the bound ThT is not necessarily shown to result in fluorescence (LeVine 1999). The fast component of the lifetime analyses, with lifetimes in the range of some hundred picoseconds, most likely corresponds to ThT sites that still allow some intramolecular flexibility leading to low fluorescence. The slow component, with lifetimes in the nanosecond range, likely originates from ThT molecules with higher fluorescence quantum yield due to tight embedding that hinders non-radiative deactivation. The relative amplitude of the slower component is $30 \%$ less in A53T (40\%) than in A30P (57 \%) templated fibrils, suggesting that the proportion of these binding sites on the two different fibril samples may be different (Table 4.2). Therefore, the observed trend of fluorescence intensity cannot be primarily due to 
differences in the fluorescence quantum efficiency of ThT when bound to fibrils of distinct morphology but has to be a result of differences in the relative distribution of ThT over the different ThT binding sites.

Simulation studies suggest grooves along phenylalanine/valine and tyrosine/leucine residues to constitute the minimal ThT binding sites (Wu, Wang et al. 2007; Wu, Biancalana et al. 2009). aSyn has two valine/phenylalanine and one leucine/tyrosine motifs. The fibril morphologies in our study are very similar to the morphologies observed in the two strains of aSyn by Heise et al. and Gath et al. (Heise, Celej et al. 2008; Gath, Bousset et al. 2014). In the latter study, amyloids were categorized as ribbons (periodic: similar to A30P fibrils) and fibrils (non-periodic: similar to A53T fibrils). The Phe/Val binding sites of ThT in $\alpha$ Syn, $\mathrm{V}_{3} \mathrm{~F}_{4}$ and $\mathrm{F}_{94} \mathrm{~V}_{95}$, according to Gath et al. are in $\beta$-strands in ribbons but not in fibrils (Figure 4.1B, C and D). Also according to Heise et al. in A53T fibrils (morphologically similar to A53T fibrils in our study), the $\mathrm{F}_{94} \mathrm{~V}_{95}$ motif is not in the $\beta$-strand. Thus, fibrils with different morphologies can have distinct boundaries of $\beta$-strands, which possibly affect the putative ThT binding sites.

Table 4.2 Table summarizing the fluorescence lifetimes determined for ThT bound to A30P and A53T templated fibrils at room temperature (RT) and $80^{\circ} \mathrm{C}$ ( \pm stdv among triplicates).

\begin{tabular}{ccccccccc}
\hline & \multicolumn{3}{c}{ A30P templated fibrils } & \multicolumn{3}{c}{ A53T templated fibrils } \\
\hline & \multicolumn{2}{c}{ Lifetimes $(n s)$} & \multicolumn{2}{c}{ Amplitude (\%) } & \multicolumn{2}{c}{ Lifetimes (ns) } & \multicolumn{2}{c}{ Amplitude (\%) } \\
& $\tau_{1}$ & $\tau_{2}$ & 1 & 2 & $\tau_{1}$ & $\tau_{2}$ & 1 & 2 \\
$R T$ & $0.31 \pm 0.04$ & $1.68 \pm 0.02$ & $43 \pm 3$ & $57 \pm 3$ & $0.22 \pm 0.01$ & $1.64 \pm 0.04$ & $60 \pm 5$ & $40 \pm 6$ \\
$80{ }^{\circ} \mathrm{C}$ & $0.12 \pm 0.01$ & $1.60 \pm 0.04$ & $78 \pm 8$ & $22 \pm 8$ & $0.13 \pm 0.03$ & $1.30 \pm 0.04$ & $78 \pm 5$ & $22 \pm 5$ \\
\hline
\end{tabular}

To further investigate the nature of the ThT binding sites and its influence on the fluorescence lifetimes we measured fibril samples at $80^{\circ} \mathrm{C}$. At elevated temperature, the dynamic equilibrium between the fibril-ThT interactions is expected to change due to effects on the relative contribution and the flexibility of the ThT binding sites. Similar changes in the samples would suggest similar binding sites on the two types of fibrils.

At $80{ }^{\circ} \mathrm{C}$ the slow component of the fluorescence lifetime of ThT bound to A30P templated fibrils changed only slightly $(1.60 \pm 0.04 \mathrm{~ns})$ but the relative amplitude dropped by $61 \%$ to $22 \%$. ThT bound to A53T templated fibrils on the other hand 
showed a $20 \%$ decrease in lifetime (1.30 $\pm 0.04 \mathrm{~ns})$ as well as nearly $50 \%$ decrease in the relative amplitude to $22 \%$. Thus at $80{ }^{\circ} \mathrm{C}$ the relative amplitudes of the slower component are similar (at $22 \%$ ) in both the samples but the lifetimes are different, with A30P templated fibrils showing longer lifetimes of $1.60 \pm 0.04 \mathrm{~ns}$ compared to $1.30 \pm$ $0.04 \mathrm{~ns}$ for A53T templated fibrils (Table 4.2). Hence, at higher temperature, the modifications in the fibril-ThT interactions are not similar; this distinctly indicates that the binding sites for ThT on the A30P and A53T templated fibrils are qualitatively different. Together, the experimentally observed differences in the lifetimes and the relative amplitudes at room temperature and $80{ }^{\circ} \mathrm{C}$ show that although the measured lifetimes at room temperature are similar, the $\mathrm{ThT}$ binding surfaces on morphologically distinct fibrils are not identical.

Since distinct fibril polymorphs show characteristic interactions with ThT that influence the fluorescence behavior of ThT, aggregations leading to different relative ratios of polymorphs are anticipated to show different fluorescence intensities. Furthermore, given the stochastic nature of nucleation and fibrillization the ratio of polymorphs even within triplicates is likely to be different. Thus, polymorph specific ThT interaction could be a cause of the routinely observed irreproducibility in ThT assays. Correspondingly, as demonstrated in this study, seeded aggregations and protocols optimized to yield homogeneous fibrils display improved reproducibility in ThT assays (Buell, Galvagnion et al. 2014; Sidhu, Segers-Nolten et al. 2014).

\section{ThT fluorescence lifetime and ThT concentration}

The results so far suggest that ThT fluorescence intensity is sensitive to the fibril morphology in an aggregation reaction. Another intrinsic factor that may affect ThT fluorescence lifetimes and intensity could be the local ThT concentration. Like most fluorophores, ThT is widely speculated to self-quench at high concentrations (Dzwolak and Pecul 2005). To probe if the concentration of ThT has any effect on the ThT fluorescence lifetimes and intensity, we titrated $100 \mu \mathrm{M}$ of wt $\alpha$ Syn fibrils against 2.5 to $100 \mu \mathrm{M}$ ThT.

The slow component of the lifetime of ThT fluorescence steadily decreased from 2.09 to $1.28 \mathrm{~ns}$ with an increase in ThT concentration (Figure 4.6A). Self-quenching observed in fluorophores is believed to be a result of collisional (dynamic) selfquenching. In case of purely collisional self-quenching, the decrease in lifetime is 
complemented with a decrease in fluorescence intensity. Titration of aSyn fibrils against ThT, followed by fluorescence intensity measurement at $485 \mathrm{~nm}$, showed an almost parallel decrease in intensity with increasing ThT concentration (Figure 4.6B). Therefore, the present results suggest that ThT undergoes collisional quenching at higher concentrations.
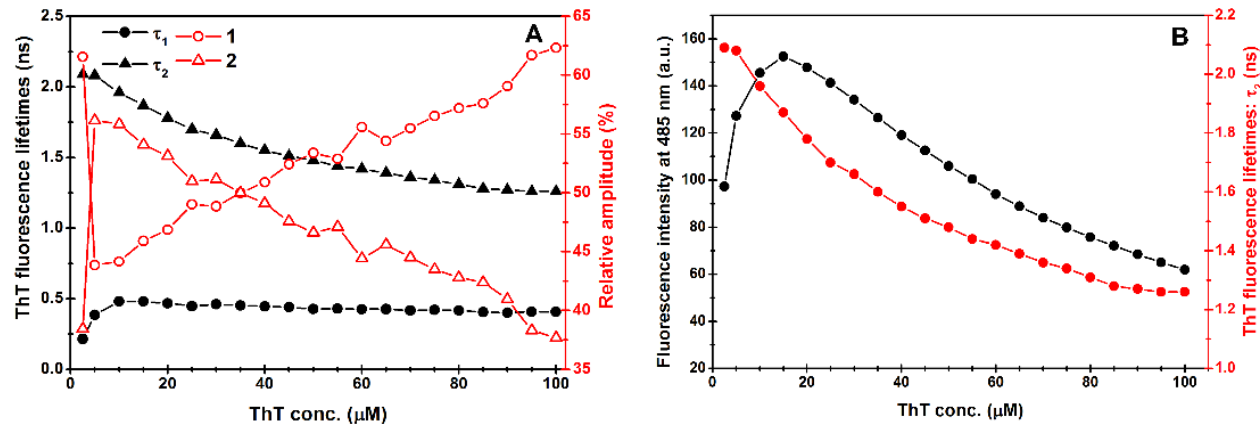

Figure 4.6 Plots showing titration of $100 \mu \mathrm{M}$ wt aSyn fibrils against ThT. (A) Changes in ThT lifetimes and their respective amplitudes with increasing ThT concentration. (B) A comparison of the changes in the lifetimes of $\tau_{2}$ and the fluorescence intensity of ThT at $485 \mathrm{~nm}$ with increasing ThT concentration.

The observed decrease in the lifetimes of ThT (slow component) fluorescence at higher concentrations is reflective of a crowded milieu where ThT molecules are in proximity and are likely to quench each other. The plateau phase in the ThT assays can be imagined to result in a similar scenario, where fibrils are prone to cluster. In such a case, ThT bound to the fibrils can be expected to undergo self-quenching, which could explain the often-observed decline in fluorescence intensity post plateau phase. Together the present study shows that the fluorescence intensity of ThT is influenced by the morphology of the fibrils as well as the ThT concentration.

\section{ThT fluorescence and fibril surface}

In AFM and EM images, amyloid fibrils often show a periodic twist (periodicity) along the length of the fibrils (Petkova, Leapman et al. 2005; Adamcik, Jung et al. 2010). The twist has been suggested to show handedness, left or right, which in turn is advocated to represent the chiral nature of the fibrils (Usov, Adamcik et al. 2013). Studies on insulin fibrils propose that chiral fibrils present chiral surfaces for molecular 
interaction. Consequently, due to the presence of chiral binding sites, ThT bound to insulin fibrils has been reported to show a pronounced CD signal around its absorption band, i.e. $450 \mathrm{~nm}$, and this phenomenon is named induced circular dichroism (ICD) (Dzwolak and Pecul 2005; Loksztejn and Dzwolak 2008). In our study, analyses of the AFM images show that A30P fibrils have a left-handed twist. The A53T fibrils however show a seemingly right-handed twist (Figure 4.7). Since fibrils have been suggested to be ubiquitously left-handed, it is likely that the observed right-handedness is an effect of sample deposition. To ascertain if the two fibrils morphologies have chiral differences and if ThT gives an ICD signal when bound to aSyn fibrils, we collected CD spectra from 350 to $500 \mathrm{~nm}$.
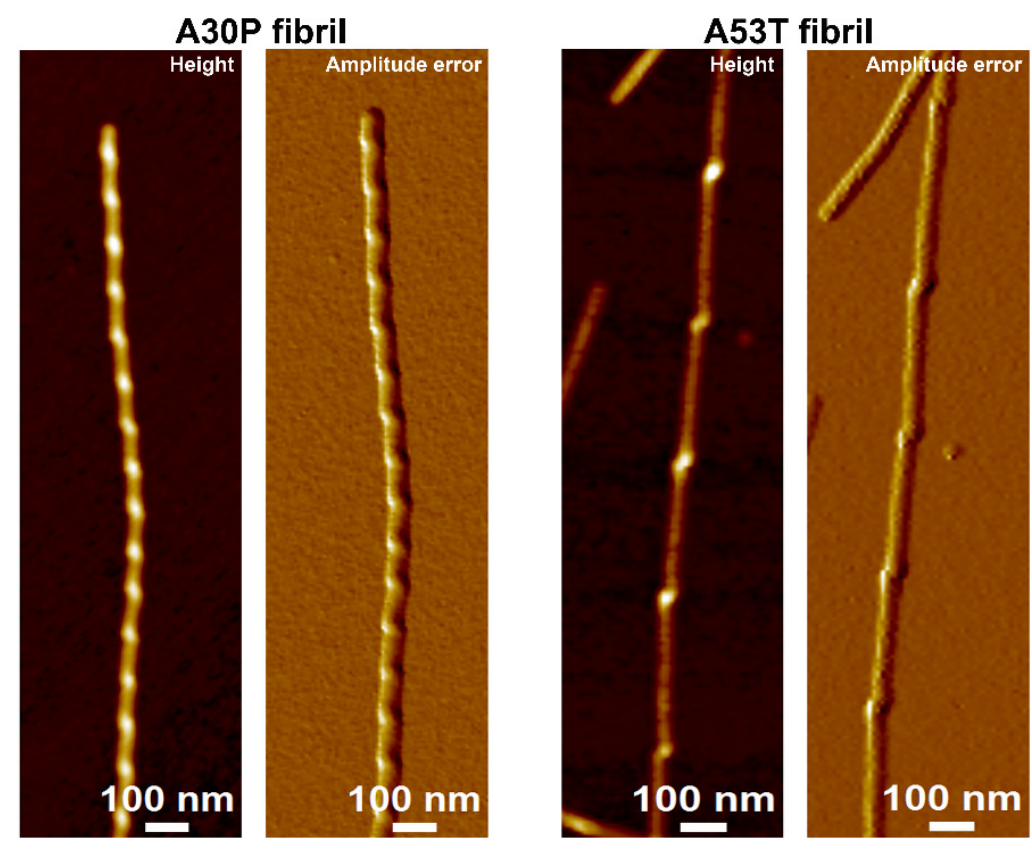

Figure 4.7 Representative AFM height and amplitude error images showing left and right handed fibrils of A30P and A53T aSyn, respectively.

The ThT solution alone did not show any ICD signal, however in the presence of fibrils (wt, A30P and A53T) a signal around $450 \mathrm{~nm}$ was observed. The sign of the signal, however, was not consistent, neither among the triplicates nor between 
consecutive spectra (Figure 4.8A). The ambiguity of the ICD signal could be due to the high concentration of fibrils that leads to interfibrillar association. The clustered fibrils present a complex and stochastically produced surface for ThT binding. To probe this hypothesis, we diluted the fibril samples and measured the CD spectra.
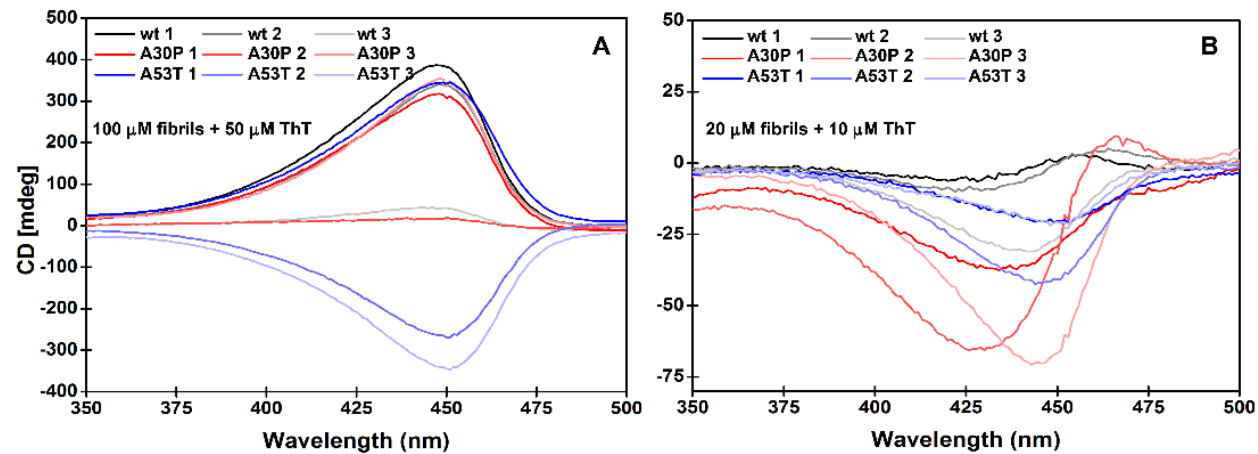

Figure 4.8 CD spectra of ThT bound to wt, A30P and A53T fibrils. (A) Triplicate of fibril sample showing inconsistent ICD signal. (B) Dilution of sample in " $A$ " results in a change to negative ICD signal in all samples.

On fivefold dilution, all the fibril samples, earlier showing mixed sign of the ICD signal, showed negative ICD spectra (Figure 4.8B). Therefore, the concentration of the fibrils may influence the ThT binding, however, the precise mechanism and what changes occur on dilution of the sample are difficult to unravel with the present data. Overall, although ThT bound to aSyn fibrils shows an ICD signal, its relation with fibril morphology and implication for ThT fluorescence intensity is not clear.

\section{CONCLUSIONS}

ThT is used as a standard probe in comparative aggregation studies that examine the effect of mutations, solution conditions, and small molecule inhibitors on amyloid aggregation kinetics. ThT exhibits enhanced fluorescence intensity on binding the $\beta$ sheet rich surface of amyloid fibrils and higher fluorescence is typically associated with higher $\beta$-sheet content, i.e. amyloid fibrils. However, our results show that the relative ThT fluorescence intensities do not always faithfully reflect the conversion of monomers into fibrils, and cannot be used as quantitative indicators of fibril mass. We 
further demonstrate that the ThT fluorescence behavior is influenced by multiple factors like the binding sites on the fibrils (dependent on fibril morphology) and ThT concentration. Thus, the modes of fibril-ThT interaction can be highly specific and most likely differ for each polymorph.

\section{ACKNOWLEDGEMENTS}

We thank Kirsten van Leijenhorst-Groener, Yvonne Kraan and Nathalie Schilderink for protein expression and purification. Dr. Christian Blum for advice and interpretation of fluorescence lifetime measurements, and Dr. Martin Bennink, Kees van der Werf and Robert Molenaar for advice on AFM. 


\section{Chapter 5}

\section{Conformational compatibility is essential for heterologous aggregation of $\alpha$-synuclein}

In aggregation prone conditions, soluble amyloidogenic protein monomers can fibrillize de novo or can be induced to fibrillize on preformed fibrillar seeds (seeded aggregations). Seeded aggregation between seeds and monomers of different conformation is shown to reproduce the morphology of the seeds. However, not all proteins are known to cross seed aggregation. In some studies, cross seeding is proposed to be restricted due to differences in the protein sequences of the seeds and the soluble monomers, while in some reports, conformational differences are proposed to be the key determinants of seeding propensity. In this chapter, we examine cross seeding between three aSyn sequences: wt, A30P and A53T, each varying in only one amino acid but forming morphologically distinct fibrils. Results from ensemble ThT measurements and single fibril fluorescence microscopy show that in the solution conditions used here, conformity between the seeds and monomers conformations is essential for seed elongation. Moreover, elongation characteristics of the seeds are defined by the type of seed.

Parts of this chapter will be published as: Arshdeep Sidhu, Ine Segers-Nolten, Vinod Subramaniam. Conformational compatibility is essential for heterologous aggregation of $\alpha$-synuclein. 


\section{INTRODUCTION}

Misfolded protein aggregates are associated with a number of degenerative diseases (Chiti and Dobson 2006). While initiation of protein aggregation in vivo is not well understood, it is believed that once initial aggregates are formed in a few cells, they persist due to resistance to degradation or aberrations in protein degradation pathways (Jucker and Walker 2013; Knowles, Vendruscolo et al. 2014; Perrett, Alexopoulou et al. 2015). Presence of misfolded proteins is suggested to induce misfolding in nascent polypeptides of same (homologous seeding) as well as of different sequences (heterologous/cross seeding) (Morales, Moreno-Gonzalez et al. 2013). Homologous as well as heterologous seeding is recognized as a key accelerator of in vivo aggregation of soluble monomers, and is proposed to be the main mechanism for the spread of the associated pathology (Harper and Lansbury 1997; Hilker, Brotchie et al. 2011; Goedert, Spillantini et al. 2013; Morales, Moreno-Gonzalez et al. 2013; Goedert, Falcon et al. 2014). In vitro seeded experiments are over-simplified models of in vivo aggregation; however, they can be instrumental in identifying factors that promote or inhibit cross seeding.

In seeded aggregations, the nucleation or lag phase is circumvented, leading to almost spontaneous exponential fibrillization of the soluble monomers (Jarrett and Lansbury 1993; Harper and Lansbury 1997; Wood, Wypych et al. 1999). Unlike homologous fibrillization, in heterologous fibrillization not all amyloidogenic proteins are known to cross seed aggregation. Sequence similarity and conformational compatibility are two principal factors suggested to govern cross seeding. In the case of hen egg lysozyme, sequence similarity has been suggested to be critical for cross seeded aggregation with other proteins (Krebs, Morozova-Roche et al. 2004). However, poor cross seeding between $A \beta$ and IAPP, which share high sequence similarity, highlights the importance of the molecular conformations of the seeds and the monomers (O'Nuallain, Williams et al. 2004). Therefore, like most other amyloid features, cross seeding characteristics vary between different amyloidogenic proteins.

Heterologous aggregation of aSyn: In PD, in addition to wt aSyn, which is implicated in idiopathic $\mathrm{PD}$, a number of point mutations are documented for the familial form. These point mutations, A30P, E46K, H50Q, G51D, A53T and A53E are reported to be autosomal dominant point mutations, where presence of a single copy of 
the mutant gene can lead to disease conditions (Polymeropoulos, Lavedan et al. 1997; Kruger, Kuhn et al. 1998; Zarranz, Alegre et al. 2004; Lesage, Anheim et al. 2013; Proukakis, Dudzik et al. 2013; Pasanen, Myllykangas et al. 2014). In heterozygous individuals, carrying a mutant allele, both wt and mutant $\alpha$ Syn are expressed. In such a situation, the presence of two different protein sequences can be imagined to modulate aggregation by cross seeding. For $\alpha$ Syn, successful cross seeding between wt $\alpha$ Syn and A30P support sequence dependence (Yonetani, Nonaka et al. 2009), while mutual enhancement of $\alpha$ Syn and tau aggregation point towards a conformation dependent cross seeding (Giasson, Forman et al. 2003; Kotzbauer, Giasson et al. 2004). In addition, a recent study investigating the effect of different strains (polymorphs) of aSyn on tau aggregation suggests strain-specific seeding efficiency, underscoring the importance of subtle structural differences in templating fibrillization (Guo, Covell et al. 2013).

In chapters 2 and 3, we showed that wt, A30P and A53T monomers can be induced to form near homogenous fibril populations. Longitudinal studies on these fibrils showed that in about 3-6 months, the wt fibrils changed into fibrils with morphologies very similar to $\mathrm{A} 53 \mathrm{~T}$ fibrils while both the disease mutant fibrils are more stable in time and do not change. Initially the morphologies of the wt fibrils were, however, comparable to the morphologies of A30P fibrils at plateau phase (Figure 5.1) (Lemkau, Comellas et al. 2012). This indicates that the wt sequence can adopt conformations similar to both A30P and A53T monomers. However, A30P and A53T mutants were not observed to form fibrils of morphologies typical for $\mathrm{A} 53 \mathrm{~T}$ and $\mathrm{A} 30 \mathrm{P}$, respectively. Assuming that in the applied solution conditions, monomers of A30P and A53T prefer select conformations that leads to formation of nearly homogeneous and specific fibril morphology, it would be interesting to examine the aggregation of these proteins in cross seeded aggregations. The principal goal is to investigate if the seeds of different conformations can act as templates for monomers that prefer a distinct conformation.

In the present chapter, we study homologous and heterologous seeded aggregations of wt, A30P and A53T. We analyze the aggregation kinetics with ThT fluorescence assays and corroborate the results with residual monomer concentration (RMC) measurements. Using two color fluorescence microscopy experiments, we illustrate the polymorph- and sequence-specific characteristics of the seeds and soluble monomeric aSyn. 


\section{MATERIALS AND METHODS}

\section{$\alpha$ Syn expression and purification}

Wt, A30P, A53T and cysteine carrying mutants of wt (A140C), A30P (A30P-A140C) and A53T (A53T-A140C) were used in the present study. The cysteine carrying mutants were generated by using QuikChange II Site-Directed Mutagenesis Kits (Agilent Technologies). All the sequences were expressed and purified using protocols described earlier (Sidhu, Segers-Nolten et al. 2014).

\section{Unlabeled $\alpha$ Syn fibrillization for monitoring kinetics from ThT fluorescence}

De novo aggregations

$250 \mu \mathrm{M}$ stocks of monomeric wt, A30P and A53T frozen at $-80{ }^{\circ} \mathrm{C}$ were thawed and fibrillized in de novo aggregation reactions with $100 \mu \mathrm{M}$ unlabeled $\alpha$ Syn, $10 \mathrm{mM}$ Tris$\mathrm{HCl}, 10 \mathrm{mM} \mathrm{NaCl}, 0.1 \mathrm{mM}$ EDTA and $20 \mu \mathrm{M} \mathrm{ThT}$, at $\mathrm{pH}$ 7.4. All reactions were prepared in triplicate with volumes of $400 \mu \mathrm{l}$ each in $2 \mathrm{ml}$ Lo-Bind round bottom Eppendorf centrifuge tubes and were incubated at $37^{\circ} \mathrm{C}$ with $500 \mathrm{rpm}$ orbital shaking in an Eppendorf Thermo-mixer comfort. The fibrils produced were checked for their morphology by AFM and were used as seeds in subsequent seeded experiments. The seeds for aggregation were prepared by sonication of the fibrils in thin walled PCR tubes for 2 minutes in a bath sonicator (Branson 1510).

Seeded aggregations: homologous and heterologous

Fibrillization reactions were setup using $2 \mu \mathrm{M}$ pre-formed fibrils as seeds and $98 \mu \mathrm{M}$ unlabeled $\alpha$ Syn, $10 \mathrm{mM}$ Tris- $\mathrm{HCl}, 10 \mathrm{mM} \mathrm{NaCl}, 0.1 \mathrm{mM}$ EDTA and $20 \mu \mathrm{M}$ ThT, at $\mathrm{pH}$ 7.4. All the reactions were prepared in triplicate in $200 \mu \mathrm{l}$ volume and incubated in 96 well plates with optically transparent bottoms (Nunc, Thermo Fisher Scientific), sealed with adhesive film (Viewseal, Greiner Bio One). The plates were incubated at $37^{\circ} \mathrm{C}$ with orbital shaking in a Safire ${ }^{2}$ microplate reader (Tecan). The aggregations were monitored using ThT fluorescence by $446 \mathrm{~nm}$ excitation and following the ThT fluorescence emission intensity (bottom reading) at $485 \mathrm{~nm}$. Readings were taken every 15 minutes.

Homologous aggregations were setup with wt/A30P/A53T seeds and wt/A30P/A53T monomers, respectively. Heterologous aggregations were setup using 
wt monomers with A30P or A53T seeds, A30P monomers with wt or A53T seeds, and A53T monomers with wt or A30P seeds.

\section{Residual monomer concentration (RMC) determination}

The RMC was determined by centrifugation of $100 \mu \mathrm{l}$ of the aggregated protein solution at $21,000 \times \mathrm{g}$ at room temperature for 1 hour in an IEC Micromax microcentrifuge (Thermo Fisher Scientific). $50 \mu \mathrm{l}$ of the supernatant was removed and the absorbances at 280 and $330 \mathrm{~nm}$ were measured on a NanoDrop ND-1000 spectrophotometer (Isogen Life Science). The absorption at $280 \mathrm{~nm}$ was corrected for scattering contributions $\left(\mathrm{A}_{330}\right)$ that possibly result from oligomeric assemblies (Grimsley 2004) before calculation of the RMC using extinction coefficients of 5600 and $5745 \mathrm{M}^{-1} \cdot \mathrm{cm}^{-1}$ for wt and cysteine carrying mutants.

\section{Atomic force microscopy (AFM)}

AFM samples were prepared by adsorbing $20 \mu \mathrm{l}$ of fibril sample, 5 to 10 times diluted in $10 \mathrm{mM}$ Tris- $\mathrm{HCl}, 10 \mathrm{mM} \mathrm{NaCl}$ at $\mathrm{pH} 7.4$ on freshly cleaved mica (Muscovite mica, V-1 quality, EMS) for 4 minutes, followed by 2 gentle washes with $100 \mu$ of fresh Milli$\mathrm{Q}$ water and drying in a gentle stream of nitrogen gas. AFM images were acquired on a Bioscope Catalyst (Bruker) in soft tapping mode in air using a silicon probe, NSC36 tip $B$ with force constant of $1.75 \mathrm{~N} / \mathrm{m}$ (NanoAndMore). All images were captured with a resolution of $512 \times 512$ pixels per image with a scan rate of $0.5 \mathrm{~Hz}$. Images were analyzed by Scanning Probe Image Processor (SPIP)-6.0.13 software (Image Metrology).

\section{Experiments with Alexa Fluor labeled $\boldsymbol{\alpha}$ Syn}

Alexa Fluor labeling of monomers

Maleimide functionalized Alexa Fluor (AF) dyes, AF 488 and AF 647, were dissolved in dry DMSO to prepare $25 \mathrm{mM}$ stocks and stored at $-80{ }^{\circ} \mathrm{C}$ in single use aliquots. For assessing the effect of different AF dyes on fibril elongation, wt monomers carrying a cysteine at position 140 (alanine exchanged for cysteine) were labeled with AF 488 or AF 647 .

$250 \mu \mathrm{M}$ stocks of monomers were thawed and reduced with freshly prepared $1 \mathrm{mM}$ DTT solution for 30 minutes at room temperature. Next, DTT was removed from the protein solution using a $2 \mathrm{ml}$ Zeba spin desalting column (Pierce Biotechnology) 
following the manufacturer's protocol. Reduced and DTT free monomers were immediately mixed with 3 molar excess of AF dye (thawed just before use). The reaction was mixed well and incubated for 1 hour at room temperature in the dark. Next, excess unreacted dye was removed by two sequential steps of desalting by $2 \mathrm{ml}$ Zeba spin columns. Stoichiometry/degree of labeling was determined by following the manufacturer's instructions, using extinction coefficients of 5745 (A140C), 71,000 (AF 488 ) and 239,000 (AF 647) $\mathrm{M}^{-1} \cdot \mathrm{cm}^{-1}$. The calculated stoichiometry was 1.1 moles of dye per mole of protein (AF 488) and 2.3 moles of dye per mole of protein (AF 647).

For the seeded aggregation experiments, monomers of cysteine carrying mutants of wt (A140C), A30P (A30P-A140C) and A53T (A53T-A140C) were labeled with AF 647 dye following the protocol described above. The stoichiometry of labeling was calculated to be 1.0, 2.2 and 1.7 moles of dye per moles of protein, for A140C, A30PA140C and A53T-A140C, respectively. Yield of labeled monomers in all reactions was about $80 \%$.

\section{Preparation of Alexa Fluor labeled fibrils}

\section{AF 488 and AF 647 labeled A140C-fibrils for length analysis}

AF labeled fibrils were prepared by seeded aggregation ( $2 \mu \mathrm{M}$ wt seeds) of $88 \mu \mathrm{M}$ unlabeled wt $\alpha$ Syn monomers, $10 \mu \mathrm{M}$ AF 488 or AF 647 labelled A140C monomers, 10 $\mathrm{mM}$ Tris- $\mathrm{HCl}, 10 \mathrm{mM} \mathrm{NaCl}, 0.1 \mathrm{mM}$ EDTA, at $\mathrm{pH}$ 7.4. All reactions were prepared in triplicate with volumes of $400 \mu \mathrm{l}$ each in $2 \mathrm{ml}$ Lo-Bind round bottom Eppendorf centrifuge tubes and were incubated at $37^{\circ} \mathrm{C}$ with $500 \mathrm{rpm}$ orbital shaking for 24 hours in an Eppendorf Thermo-mixer comfort.

\section{AF 647 labeled fibrils for use as seeds}

AF labeled fibrils were prepared by fibrillization of $88 \mu \mathrm{M}$ unlabeled wt/A30P/A53T aSyn monomers, $10 \mu \mathrm{M}$ AF 647 labelled A140C/A30P-A140C/A53T-A140C monomers, $10 \mathrm{mM}$ Tris- $\mathrm{HCl}, 10 \mathrm{mM} \mathrm{NaCl}, 0.1 \mathrm{mM}$ EDTA, at $\mathrm{pH} 7.4$ in the presence of $2 \mu \mathrm{M} \mathrm{wt} / \mathrm{A} 30 \mathrm{P} / \mathrm{A} 53 \mathrm{~T}$ seeds, respectively . All reactions were prepared in triplicate with volumes of $400 \mu \mathrm{l}$ each in $2 \mathrm{ml}$ Lo-Bind round bottom Eppendorf centrifuge tubes and were incubated at $37^{\circ} \mathrm{C}$ with $500 \mathrm{rpm}$ orbital shaking for 72 hours in an Eppendorf Thermo-mixer comfort. 


\section{Seeded aggregations: homologous and heterologous}

Seeded aggregations were performed by combining $10 \mu \mathrm{M}$ (based on initial monomer concentration) AF 647 labeled seeds and $90 \mu \mathrm{M}$ unlabeled monomers. Aggregations were setup in $10 \mathrm{mM}$ Tris- $\mathrm{HCl}, 10 \mathrm{mM} \mathrm{NaCl}, 0.1 \mathrm{mM}$ EDTA, at $\mathrm{pH}$ 7.4. All reactions were prepared in $400 \mu \mathrm{l}$ volume in $2 \mathrm{ml}$ Lo-Bind round bottom Eppendorf centrifuge tubes and were incubated at $37^{\circ} \mathrm{C}$ in quiescent conditions for 24 hours.

Homologous aggregations were setup with wt/A30P/A53T seeds and wt/A30P/A53T monomers, respectively. Heterologous aggregations were setup using wt monomers with A30P or A53T seeds, A30P monomers with wt or A53T seeds, and A53T monomers with wt or A30P seeds.

\section{Fluorescence microscopy}

Fibrils produced in the seeded aggregations were imaged by fluorescence microscopy. The seeds were visualized using AF 647 fluorescence while the extensions were imaged through ThT fluorescence. Samples for fluorescence microscopy were prepared by placing $5 \mu \mathrm{l}$ of 50 to 100 times diluted fibril samples containing $10 \mu \mathrm{M}$ ThT on a clean microscope slide and covered by a clean cover slip. The edges of the samples were sealed with lacquer and the samples were allowed to settle for 24 hours. Fluorescence images were acquired in widefield mode using a Plan-Apo oil objective with $100 \times$ magnification ( $\mathrm{NA}=1.45)$ from Olympus on a Nikon Eclipse Ti microscope equipped with a mercury arc lamp (Nikon Intensilight, C-HGFIE) for excitation. For imaging of AF 647 a filter cube consisting of a 590/650 nm band pass excitation filter, a $660 \mathrm{~nm}$ long pass dichroic beam splitter and a 662/737 nm band pass emission filter was used. For imaging of ThT, a filter cube composed of a $445 / 465 \mathrm{~nm}$ band pass excitation filter, a $458 \mathrm{~nm}$ long pass dichroic beam splitter and a $470 / 500 \mathrm{~nm}$ band pass emission filter was used. Fibril length analysis on fluorescence images was done using Simple Neurite tracer-Fiji software (Longair, Baker et al. 2011).

\section{RESULTS AND DISCUSSION}

\section{Fibrillization of wt and the disease mutants}

Wt and the disease mutants of aSyn (A30P and A53T) were fibrillized de novo, in 10 $\mathrm{mM}$ Tris- $\mathrm{HCl}, 10 \mathrm{mM} \mathrm{NaCl}$, and $0.1 \mathrm{mM}$ EDTA, at $\mathrm{pH} 7.4$, to be used as seeds. The 
morphologies of the produced fibrils were determined by AFM imaging. Consistent with the results of chapter 3, wt fibrils showed a periodic twist $(p)$ of about $100 \mathrm{~nm}$. A30P and A53T fibrils also exhibited the morphology that is characteristic for the applied solution conditions with $p \approx 100 \mathrm{~nm}$ (A30P) and $p \approx 350 \mathrm{~nm}$ (A53T) (Figure 5.1).
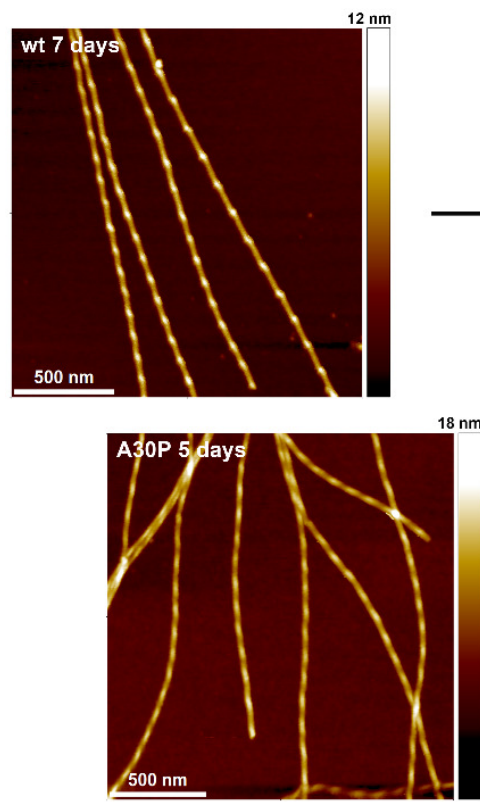
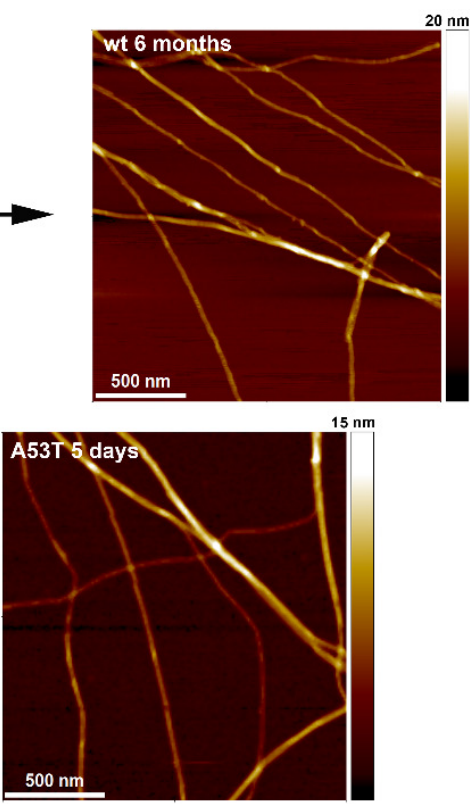

Figure 5.1 Representative AFM height images of aSyn fibrils. Upper panel, left: wt fibrils at plateau phase show periodicities $(p)$ of about 100 to $150 \mathrm{~nm}$. Upper panel, right: wt fibril samples aged around 6 months show longer $p \approx 350 \mathrm{~nm}$ with heterogeneous and non-periodic fibrils. Lower panel: the A30P fibrils at plateau phase predominantly show a $p \approx 100 \mathrm{~nm}$ (similar to wt plateau phase) while A53T fibrils at plateau phase mainly show larger $p \approx 350 \mathrm{~nm}$ with heterogeneous and non-periodic fibrils (similar to aged wt fibrils).

\section{Aggregation kinetics of $\alpha$ Syn in homologous and heterologous fibrillization}

Elongation of aSyn seeds is proposed to occur primarily through addition of monomers to the existing ends (Buell, Galvagnion et al. 2014). The extent and the rate of elongation in seeded aggregation can thus be expected to reflect the efficiency with which the soluble monomer can be incorporated at the end of the seeds. Results from chapters 3 and 4 suggest that physico-chemical and conformational differences most probably 
underlie the morphological differences observed in the AFM images of aSyn fibrils. Moreover, fibrillization of monomers of A30P and A53T aSyn into fibrils with mutually exclusive morphologies indicates a strong sequence based conformation bias. Thus, monomers of A30P and A53T may not fibrillize equally well on seeds of different conformation. To probe this hypothesis, we performed homologous and heterologous seeded aggregations with wt, A30P and A53T aSyn. Homologous aggregations were set up with wt/A30P/A53T seeds and wt/A30P/A53T monomers, respectively. Heterologous aggregations were set up using wt monomers with A30P or A53T seeds, A30P monomers with wt or A53T seeds, and A53T monomers with wt or A30P seeds.
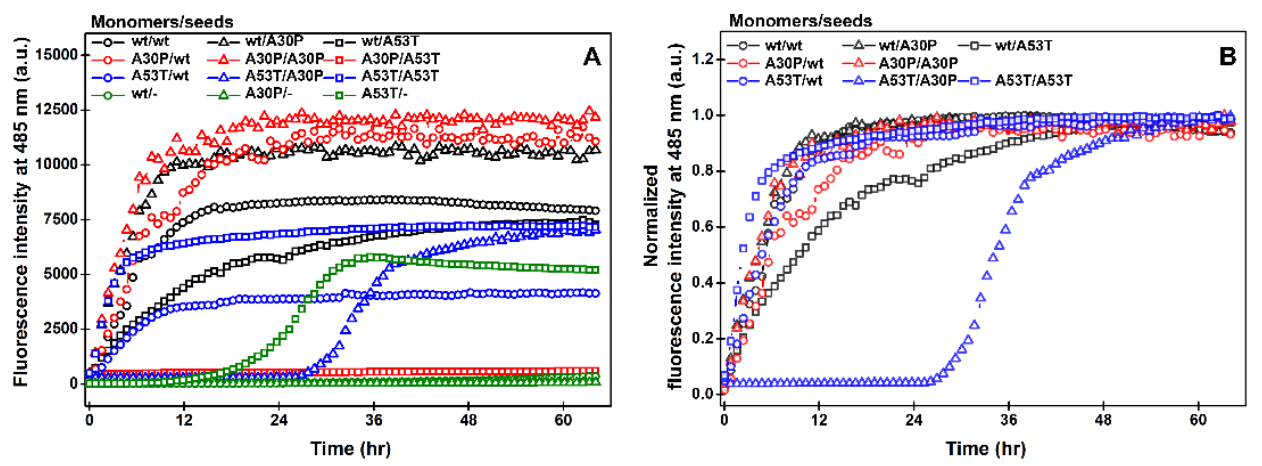

Figure 5.2 ThT fluorescence representing the kinetics of homologous and heterologous seeded aggregations. (A) Measured fluorescence intensities. Unseeded aggregations are included as reference. (B) Fluorescence intensities of the seeded aggregations, normalized to the maximum intensity measured at the plateau phase per curve. A30P/A53T aggregation curve is not shown because the plateau phase had not been reached. For graph clarity, only one representative curve from the triplicate is shown for each aggregation reaction.

\section{Homologous aggregations}

In homologous aggregations, all the reactions lacked the lag phase, which is characteristic of a seeded aggregation. The kinetics of the aggregations were quantified from triplicates by reading the time required to reach half of the maximum fluorescence intensity (half-time: $t_{1 / 2}$ ). A53T homologous aggregations showed the shortest $t_{1 / 2}$ of 2.3 \pm 0.2 hours, followed by A30P with $4.0 \pm 0.2$ hours and wt with $5.4 \pm 0.4$ hours. (Figure 5.2 and Table 5.1). 
Table 5.1 Summary of the determined half-time for homologous and heterologous seeded aggregations in hours ( \pm stdv among triplicates).

\begin{tabular}{ccccc}
\hline $\mathbf{t}_{1 / 2}$ & \multicolumn{4}{c}{ Monomers } \\
\hline & & wt & A30P & A53T \\
Seeds & wt & $5.4 \pm 0.4$ & $5.7 \pm 0.2$ & $6.2 \pm 1.2$ \\
& A30P & $4.3 \pm 0.2$ & $4.0 \pm 0.2$ & $35.0 \pm 1.5$ \\
& A53T & $9.7 \pm 1.0$ & - & $2.3 \pm 0.2$ \\
\hline
\end{tabular}

\section{Heterologous aggregations}

In heterologous aggregations, wt monomers fibrillized faster on A30P seeds than on A53T seeds $\left(\mathrm{t}_{1 / 2}=4.3 \pm 0.2\right.$ and $9.7 \pm 1.0$ hours, respectively). A30P monomers aggregated on wt seeds with a $t_{1 / 2}$ of $5.7 \pm 0.2$ hours, while they did not appear to grow at all on A53T seeds (based on the ThT assay up to the experimental time of 65 hours). A53T monomers fibrillized in the presence of wt seeds with a $t_{1 / 2}$ of $6.2 \pm 1.2$ hours. Aggregation of A53T monomers with A30P seeds showed a clear lag phase, which is aberrant for a seeded aggregation (Figure 5.2). The lag phase was determined to be 26.2 \pm 3.1 hours by the intercept of the extrapolated baseline and the slope of the exponential phase. The $t_{1 / 2}$ determined for the A53T monomer/A30P seed aggregation was $35.0 \pm 1.5$ hours (Table 5.1). Thus, based on the ThT fluorescence intensity, in heterologous reactions, wt monomers fibrillized with both $\mathrm{A} 30 \mathrm{P}$ and $\mathrm{A} 53 \mathrm{~T}$ seeds while the two mutant monomers aggregated only on wt seeds. However, it is notable that between homologous and heterologous aggregations all 3 aSyn seeds grow fastest with homologous monomers, thus indicating more efficient elongation of seeds with monomers that have same sequence and secondary/tertiary structure.

\section{Residual monomer concentration}

In chapter 4, we have shown that ThT assays alone are not reliable indicators of the extent of amyloid formation in fibrillization reactions. Thus, to ascertain that the relative fibrillization curves, obtained with ThT assays, correlate with the conversion of monomers into fibrils, we determined the RMC for all the aggregation reactions.

Based on the amount of soluble monomers present at the end of an aggregation reaction the extent of monomer incorporation into the fibrils can be determined. We calculated the RMC by measuring the absorbance at $280 \mathrm{~nm}$, including a correction for 
the contribution of soluble oligomers based on the scatter signal detected at $330 \mathrm{~nm}$ (Grimsley 2004).

In homologous aggregations, wt and A53T samples showed a conversion of about $92 \%$ of the monomers into fibrils (Table 5.2). A30P samples revealed a lower conversion of about $79 \%$, though the final fluorescence intensity of the A30P sample is considerably higher than that of wt or A53T fibrils (Figure 5.2A). This observation again highlights that the total fluorescence intensity in ThT assays can be misleading (chapter $4)$.

Table 5.2 RMC for homologous and heterologous aggregation reactions in micro molars $( \pm$ stdv among triplicates).

\begin{tabular}{lcccc}
\hline RMC & \multicolumn{4}{c}{ Monomers } \\
\hline \multirow{4}{*}{ Seeds } & wt & $8 \pm 2$ & $23 \pm 2$ & $3 \pm 3$ \\
& A30P & $20 \pm 3$ & $21 \pm 4$ & $6 \pm 1$ \\
& A53T & $25 \pm 1$ & $82 \pm 2$ & $8 \pm 2$ \\
\hline
\end{tabular}

In heterologous aggregations, consistent with the ThT results, the wt monomers fibrillized on A30P seeds and A53T seeds well with about 80 and $75 \%$ monomer conversion. Around $77 \%$ of the A30P monomers added on the wt seeds while most of the A30P monomers $(\approx 82 \%)$ with A53T seeds remained soluble. The inability of A53T seeds to seed aggregation of A30P monomers observed in ThT assays and RMC determinations is suggestive of an incompatibility between the seed conformation and the conformations favored by the soluble monomers. A53T monomers showed a high incorporation into fibrils with wt seeds $(\approx 97 \%)$ and with A30P seeds $(\approx 94 \%)$. Conversion of most of the A53T monomers (94\%) into fibrils by growing on A30P seeds is however unlikely, since the ThT curve shows a distinct lag phase. The lag phase for A53T monomers and A30P seeded aggregation is longer than the lag phase observed for unseeded (negative control) A53T aggregation ( $\approx 18$ hours). Therefore, it is possible that the fibrillization observed in this seeded aggregation is due to de novo aggregation of A53T monomers and not because of fibrillization of A53T monomers on A30P seeds. To test this assumption, we determined if the fibrils in the seeded aggregation had the typical morphology of A53T fibrils. 


\section{AFM imaging: fibril morphology}

AFM images showed that the A30P and A53T fibrils used as seeds in these experiments had the distinct morphologies of A30P and A53T fibrils (Figure 5.1). Seeded aggregations in $A \beta$ and $\alpha$ Syn have been proposed to reproduce the morphology of the seeds (Petkova, Leapman et al. 2005; Bousset, Pieri et al. 2013). In chapter 4, we show that $\mathrm{A} 30 \mathrm{P}$ and $\mathrm{A} 53 \mathrm{~T}$ seeds act as morphological templates in fibril elongation across 2 generations of seeding. Therefore, if the fibrils formed in the A53T monomer/A30P seeded aggregation are generated due to templating by the seed they should display the morphology characteristic of A30P fibrils (i.e. with $p \approx 100 \mathrm{~nm}$ ). However, if the fibrils arise due to de novo fibrillization of $\mathrm{A} 53 \mathrm{~T}$ monomers, they should exhibit the morphology typical for A53T fibrils in the given aggregation conditions $(p \approx 350 \mathrm{~nm}$ and heterogeneous fibrils). Fibrils from the A53T monomer/A30P seeded aggregations exhibit the morphology of A53T fibrils and not of A30P fibrils (Figures 5.1 and 5.3). Therefore, the fibrils are formed by de novo fibrillization of monomeric A53T and not by templated growth on the A30P seeds. This result explains the lag phase observed in the ThT assays. It also shows that like A30P monomers, which do not fibrillize on A53T seeds, A53T monomers do not elongate the A30P seeds. The marked selectivity of fibrils to act as seeds again points toward incompatibility between the conformations at the seed ends and the conformations favored by the soluble monomers. To gain a better
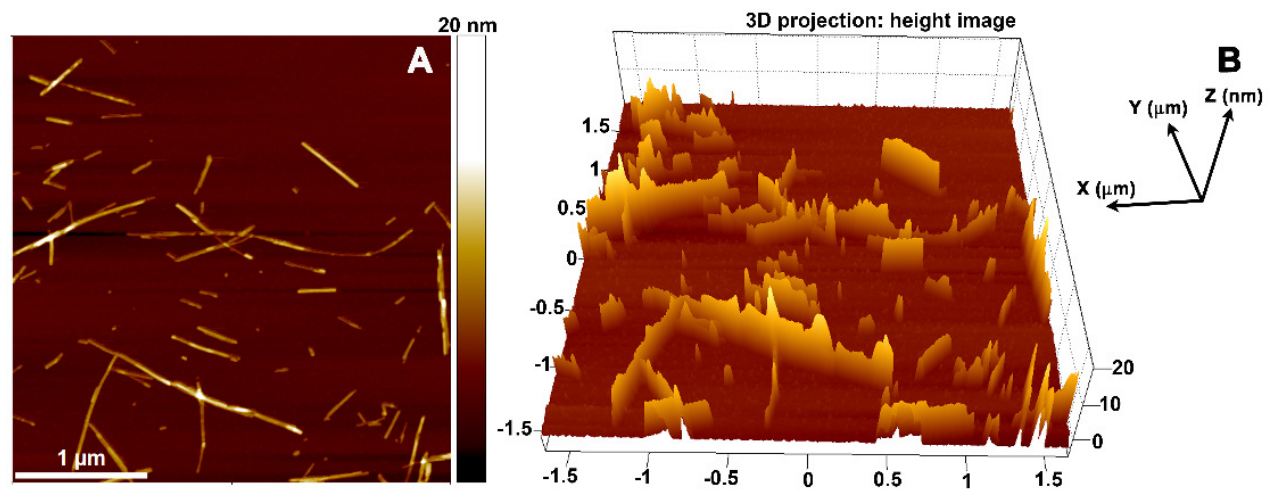

Figure 5.3 AFM height image of A53T monomers aggregated in the presence of A30P seeds. The fibrils show morphology typical for A53T fibrils. 
understanding of the seed elongation with respect to the seed and monomer compatibility we imaged fibrils by fluorescence microscopy to assess fibril growth at single fibril level.

\section{Fluorescence microscopy}

\section{Choice of fluorophore}

Visualization of amyloid fibrils by fluorescence microscopy routinely employs fibrils covalently labeled with fluorophores. The Alexa Fluor (AF) fluorophores series are one of the most commonly used fluorescent probes and are available with a variety of linkers. Previously, amyloid fibrils labeled with different AF dyes have been used to study the growth symmetry at both ends during elongation (Heldt, Zhang et al. 2011; Pinotsi, Buell et al. 2014). For our fluorescence experiments, which aim at probing a possible preference for specific monomer conformations on seed elongation, it is important to exclude a fluorophore dependent effect as much as possible. Therefore, we first prepared AF 488 and AF 647 labeled A140C fibrils under identical solution and aggregation conditions and compared their length distributions. The labeled fibrils were prepared in a seeded aggregation ( $2 \mu \mathrm{M}$ wt seeds) from a mixture of $10 \mu \mathrm{M} \mathrm{AF}$ labeled A140C monomers and $88 \mu \mathrm{M}$ unlabeled wt monomers. The mean length of the fibrils labeled with AF $488(1.2 \pm 1.0 \mu \mathrm{m})$ was about $1 \mu \mathrm{m}$ shorter than of the AF 647 labeled fibrils $(2.1 \pm 1.2 \mu \mathrm{m})$ (Figure 5.4). Although the mean fibril lengths were not decidedly different with the AF dyes used, the results indicate that a potential dye effect
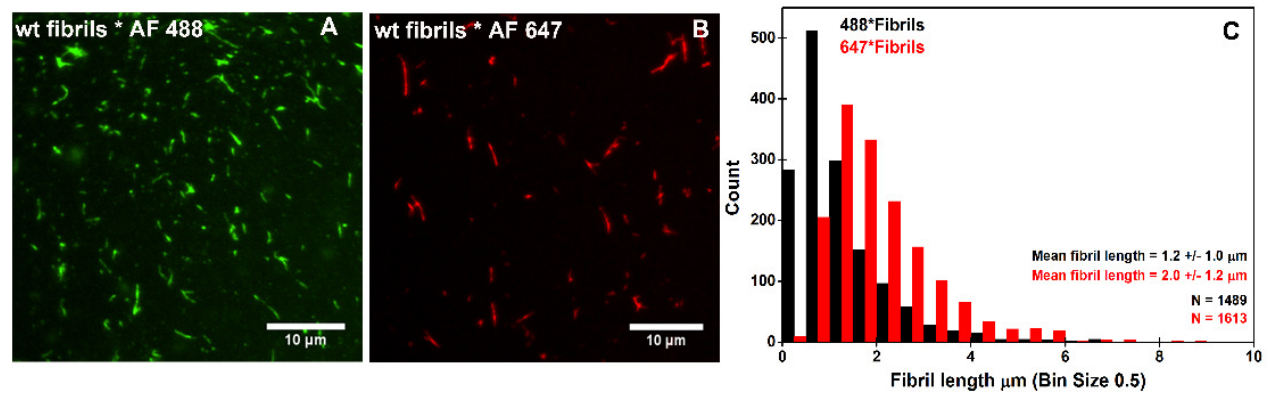

Figure 5.4 Representative widefield fluorescence microscopy images of wt $\alpha$ Syn fibrils labeled with (A) AF 488 and (B) AF 647. (C) Histogram showing length distribution of fibrils labeled with the two fluorophores. 
cannot be ruled out. Therefore, to avoid fluorophore biased fibril elongation, we decided to only make use of AF 647 labeled seeds in combination with unlabeled monomers and to image the grown extensions by fluorescence of ex situ bound ThT.

\section{Fibril length analyses}

$\mathrm{Wt}, \mathrm{A} 30 \mathrm{P}$ and A53T monomers carrying a cysteine residue at the C-terminus (substituting alanine at position 140) were labeled with AF 647 dye. $10 \mu \mathrm{M}$ labeled monomers were aggregated with $90 \mu \mathrm{M}$ unlabeled monomers of the same sequence in $10 \mathrm{mM}$ Tris- $\mathrm{HCl}, 10 \mathrm{mM} \mathrm{NaCl}$ and $0.1 \mathrm{mM}$ EDTA at $\mathrm{pH} 7.4$ to produce fibrils to be used as seeds. Next, homologous and heterologous aggregations were set up in quiescent conditions to avoid fibril fragmentation. After 24 hours, samples were prepared for widefield fluorescence microscopy (Figure 5.5). The images were analyzed for fibrillization of the monomers by unidirectional or bidirectional growth of the seed, for seeds with no elongation and for fibrils without seeds. In all the samples, about 1 $\mathrm{mm}$ of total length of the fibrils were analyzed.
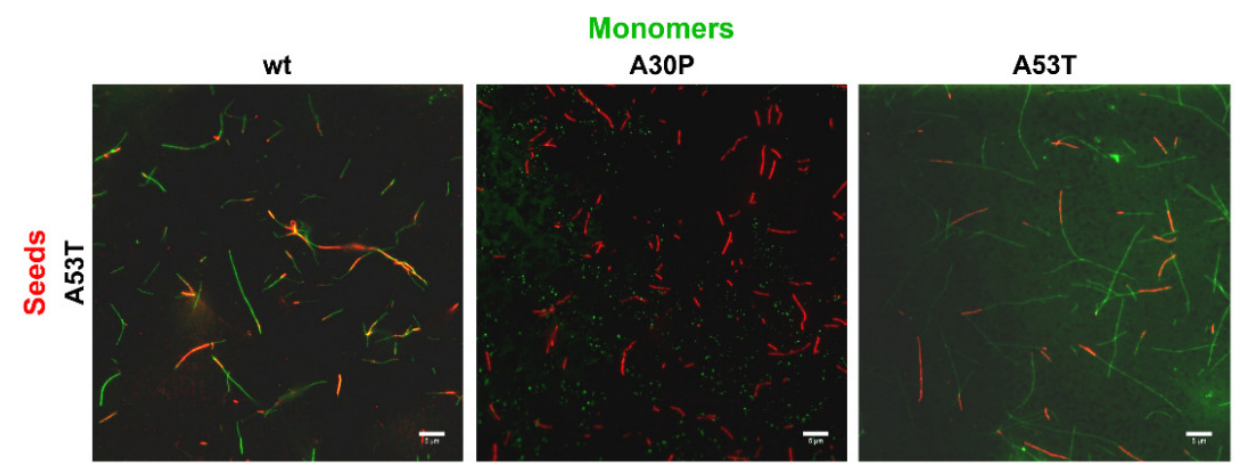

Figure 5.5 Representative overview images of A53T seeded aggregations with wt, A30P and A53T monomers, showing seeds labeled with AF 647 and extensions visualized by ex situ bound ThT. Scale bar: $5 \mu \mathrm{m}$.

\section{Extension of seeds shows sequence dependent bias}

\section{Homologous aggregations}

In homologous aggregations, 40 to $50 \%$ of the total (new) fibril length was linked to the seeds while about $30 \%$ of the fibril lengths did not show presence of any seed (Figure 5.6). The fibrils that are not associated with seeds are most likely fragments of the 
extensions grown on seeds because none of the aSyn sequences studied shows de novo fibrillization in 24 hours in quiescent aggregation conditions. A larger percentage of seeds appear to participate in fibrillization in A30P and A53T aggregations, in comparison to wt seeds, as only 5 and $3 \%$ of the total fibril lengths correspond to seeds with no extensions. Wt aggregation showed a higher percentage, $14 \%$ of the lengths, of unextended seeds, apparently correlating with relatively slower aggregation observed in the ThT assay (Figure 5.2 and 5.6). However, since the precise fibril concentration in the samples is unknown it is difficult to draw accurate conclusions on the fraction of seeds that elongate. The average length of the seed extension for wt fibrils was $9.0 \pm 3.4$ $\mu \mathrm{m}$. The average length for A30P and A53T aggregations were similar at $9.9 \pm 7.1$ and $7.5 \pm 5.1 \mu \mathrm{m}$, respectively. The length distribution for A30P and A53T extension was, however, broader in comparison to wt (Table 5.3).

Table 5.3 Average lengths of the fibrils (in $\mu \mathrm{m}$ ) measured in different seeded aggregations ( \pm stdv). Number of fibrils $(\mathrm{N})$ measured for each fibril length are shown in parentheses.

\begin{tabular}{|c|c|c|c|c|c|c|c|}
\hline $\begin{array}{l}\text { Monomers/ } \\
\text { seeds }\end{array}$ & $\begin{array}{l}\text { S.l with } \\
\text { no extn }\end{array}$ & $\begin{array}{l}\text { S.l with } 1 \\
\text { extn }\end{array}$ & E1 & $\begin{array}{l}\text { F.l with } \\
\text { no seeds }\end{array}$ & $\begin{array}{c}\text { S.l with } 2 \\
\text { extn }\end{array}$ & E1 & E 2 \\
\hline$W t / w t$ & $\begin{array}{c}2.7 \pm 2.7 \\
\quad(58)\end{array}$ & $\begin{array}{c}1.8 \pm 1.2 \\
(48)\end{array}$ & $\begin{array}{c}9.0 \pm 3.4 \\
(48)\end{array}$ & $\begin{array}{c}6.3 \pm 3.9 \\
(54)\end{array}$ & $\begin{array}{c}1.6 \pm 1.7 \\
(5)\end{array}$ & $\begin{array}{c}7.9 \pm 2.5 \\
(5)\end{array}$ & $\begin{array}{l}5.1 \pm 3.0 \\
\quad(5)\end{array}$ \\
\hline$A 30 P / w t$ & $\begin{array}{c}2.5 \pm 1.5 \\
(29)\end{array}$ & $\begin{array}{c}1.8 \pm 1.1 \\
(34)\end{array}$ & $\begin{array}{c}8.0 \pm 7.3 \\
(34)\end{array}$ & $\begin{array}{c}8.7 \pm 6.4 \\
(39)\end{array}$ & $\begin{array}{c}1.2 \pm 0.7 \\
(19)\end{array}$ & $\begin{array}{c}9.1 \pm 5.3 \\
(19)\end{array}$ & $\begin{array}{c}3.3 \pm 3.7 \\
(19)\end{array}$ \\
\hline$A 53 T / w t$ & $\begin{array}{c}3.1 \pm 0.7 \\
\quad(17)\end{array}$ & $\begin{array}{c}1.7 \pm 1.5 \\
(20)\end{array}$ & $\begin{array}{c}13.4 \pm 7.8 \\
(20)\end{array}$ & $\begin{array}{c}9.8 \pm 7.3 \\
(61)\end{array}$ & $\begin{array}{c}1.4 \pm 0.4 \\
(13)\end{array}$ & $\begin{array}{c}7.2 \pm 4.3 \\
(13)\end{array}$ & $\begin{array}{c}3.4 \pm 1.1 \\
(13)\end{array}$ \\
\hline$W t / A 30 P$ & N.D. & $\begin{array}{c}3.5 \pm 2.3 \\
(38)\end{array}$ & $\begin{array}{c}15.6 \pm 9.9 \\
(38)\end{array}$ & $\begin{array}{c}14.8 \pm 8.6 \\
(24)\end{array}$ & $\begin{array}{l}2.1 \\
(1)\end{array}$ & $\begin{array}{c}18.6 \\
(1)\end{array}$ & $\begin{array}{c}10.5 \\
(1)\end{array}$ \\
\hline A30P/A30P & $\begin{array}{c}2.5 \pm 2.0 \\
(20)\end{array}$ & $\begin{array}{c}2.9 \pm 2.1 \\
(48)\end{array}$ & $\begin{array}{c}9.9 \pm 7.1 \\
(48)\end{array}$ & $\begin{array}{c}10.7 \pm 6.3 \\
(45)\end{array}$ & $\begin{array}{c}4.0 \pm 1.7 \\
\text { (2) }\end{array}$ & $\begin{array}{c}5.1 \pm 3.1 \\
\text { (2) }\end{array}$ & $\begin{array}{c}1.3 \pm 0.1 \\
\text { (2) }\end{array}$ \\
\hline$A 53 T / A 30 P$ & $\begin{array}{c}5.2 \pm 3.4 \\
(203)\end{array}$ & N.D. & N.D. & N.D. & N.D. & N.D. & N.D. \\
\hline$W t / A 53 T$ & $\begin{array}{c}3.2 \pm 1.9 \\
(37)\end{array}$ & $\begin{array}{c}2.5 \pm 2.0 \\
(76)\end{array}$ & $\begin{array}{c}3.9 \pm 2.2 \\
(76)\end{array}$ & $\begin{array}{c}3.6 \pm 2.0 \\
(49)\end{array}$ & $\begin{array}{c}2.5 \pm 1.8 \\
(40)\end{array}$ & $\begin{array}{c}4.8 \pm 2.0 \\
(40)\end{array}$ & $\begin{array}{c}2.5 \pm 1.4 \\
(40)\end{array}$ \\
\hline A30P/A53T & $\begin{array}{c}4.0 \pm 3.0 \\
(236)\end{array}$ & N.D. & N.D. & N.D. & N.D. & N.D. & N.D. \\
\hline A53T/A53T & $\begin{array}{c}4.3 \pm 2.5 \\
(8)\end{array}$ & $\begin{array}{c}2.9 \pm 2.2 \\
(38)\end{array}$ & $\begin{array}{c}7.5 \pm 5.1 \\
(38)\end{array}$ & $\begin{array}{c}6.4 \pm 3.5 \\
(51)\end{array}$ & $\begin{array}{c}3.1 \pm 2.0 \\
(18)\end{array}$ & $\begin{array}{c}8.8 \pm 5.3 \\
(18)\end{array}$ & $\begin{array}{c}2.8 \pm 1.9 \\
(18)\end{array}$ \\
\hline
\end{tabular}

S.l: seed length, F.l: fibril length, N.D.: not detected, extn: extension, E1: length of extension 1, E2: length of extension 2 


\section{Heterologous aggregations}

In heterologous aggregations, wt monomers appeared to grow well on both $\mathrm{A} 30 \mathrm{P}$ and A53T seeds with close to $50 \%$ of the total fibril lengths linked with growth on the seeds. However, the average length of a wt fibril grown on an A30P seed is almost 5 times (15.6 $\pm 9.9 \mu \mathrm{m})$ the average length of a wt fibril grown on an A53T seed $(3.9 \pm 2.2 \mu \mathrm{m})$. The A30P monomers fibrillized on wt seeds account for $\approx 50 \%$ of the total fibril lengths. In aggregation with A53T monomers and wt seeds $\approx 37 \%$ of the fibril lengths were associated with seeds and around $54 \%$ did not show any seed. In agreement with the ThT assay, RMC and AFM imaging, A30P and A53T seeds did not show any elongation in the presence of $\mathrm{A} 53 \mathrm{~T}$ and $\mathrm{A} 30 \mathrm{P}$ monomers, respectively (Figures 5.5 and 5.6 and Table 5.3). These results establish that the conformations of A30P and A53T seeds are not conducive for sustained addition and elongation of A53T and A30P monomers, respectively. In light of the dock and lock mechanism for the elongation of fibrils (Esler, Stimson et al. 2000; Straub and Thirumalai 2011; Qiang, Kelley et al. 2013), it is probable that these monomers tether at the ends of the seeds (lock) but are unable to fold into growth favoring conformations. Thus, the elongation is blocked altogether.
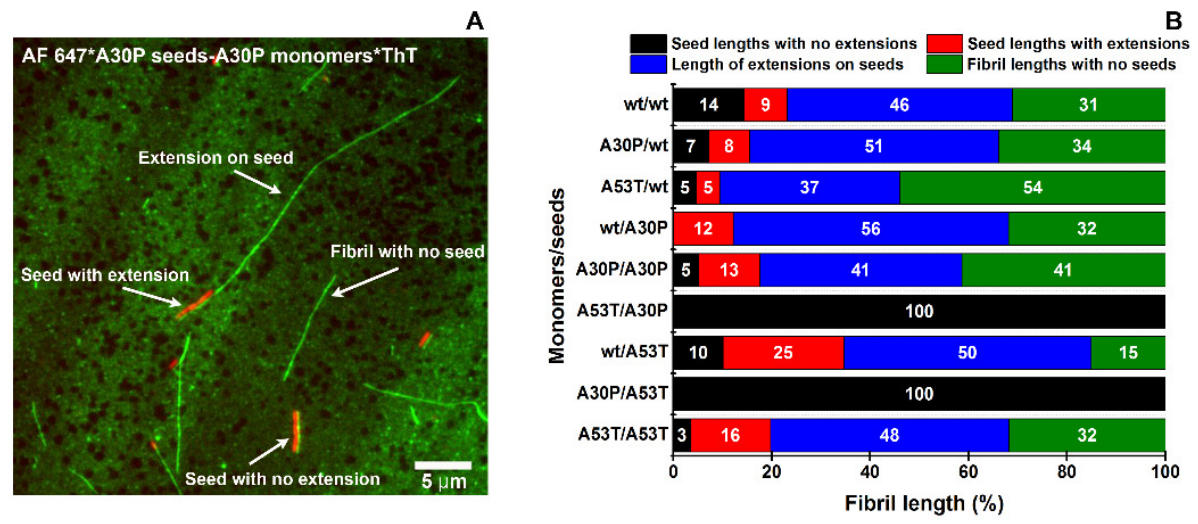

Figure 5.6 (A) Representative widefield fluorescence image exemplifying the various types of fibril lengths analyzed. Seeds were labeled with AF 647 and the extensions were visualized by ex situ binding of ThT. Small red-green color shifts result from a slightly different optical path when using different filter cubes. Scale bar: $5 \mu \mathrm{m}$ (B) Histogram showing percentage lengths of the fibrils in different seeded aggregation reactions. Around $1 \mathrm{~mm}$ of total length was measured for all the aggregations. For seeds with bidirectional growth, both the extensions were included in the analyses as "length of extensions on seeds". 


\section{Kinetics of seed extension is heterogeneous}

An evident feature of the seed elongation, irrespective of the monomers, is the broad distribution of the fibril lengths per seed (Figure 5.7). This broad distribution suggests heterogeneous rates of seed elongation. Heterogeneity in the elongation rates has been proposed to be a consequence of the "stop and go" growth mechanism (Ban, Hoshino et al. 2004; Hoyer, Cherny et al. 2004; Qiang, Kelley et al. 2013; Pinotsi, Buell et al. 2014; Wordehoff, Bannach et al. 2015). The stop and go mechanism outlines the possible eventualities of the docking step in the lock and dock mechanism of growth. Once a monomer is docked at the fibril end, it may acquire the template fold and contribute to the fibril growth ("go") or it may fail to do so and stall the growth intermittently or permanently ("stop"). In case of intermittent stalling of the elongation, the growth rate at each end of the seed could be different, leading to heterogeneous growth rates, as observed in the present study.

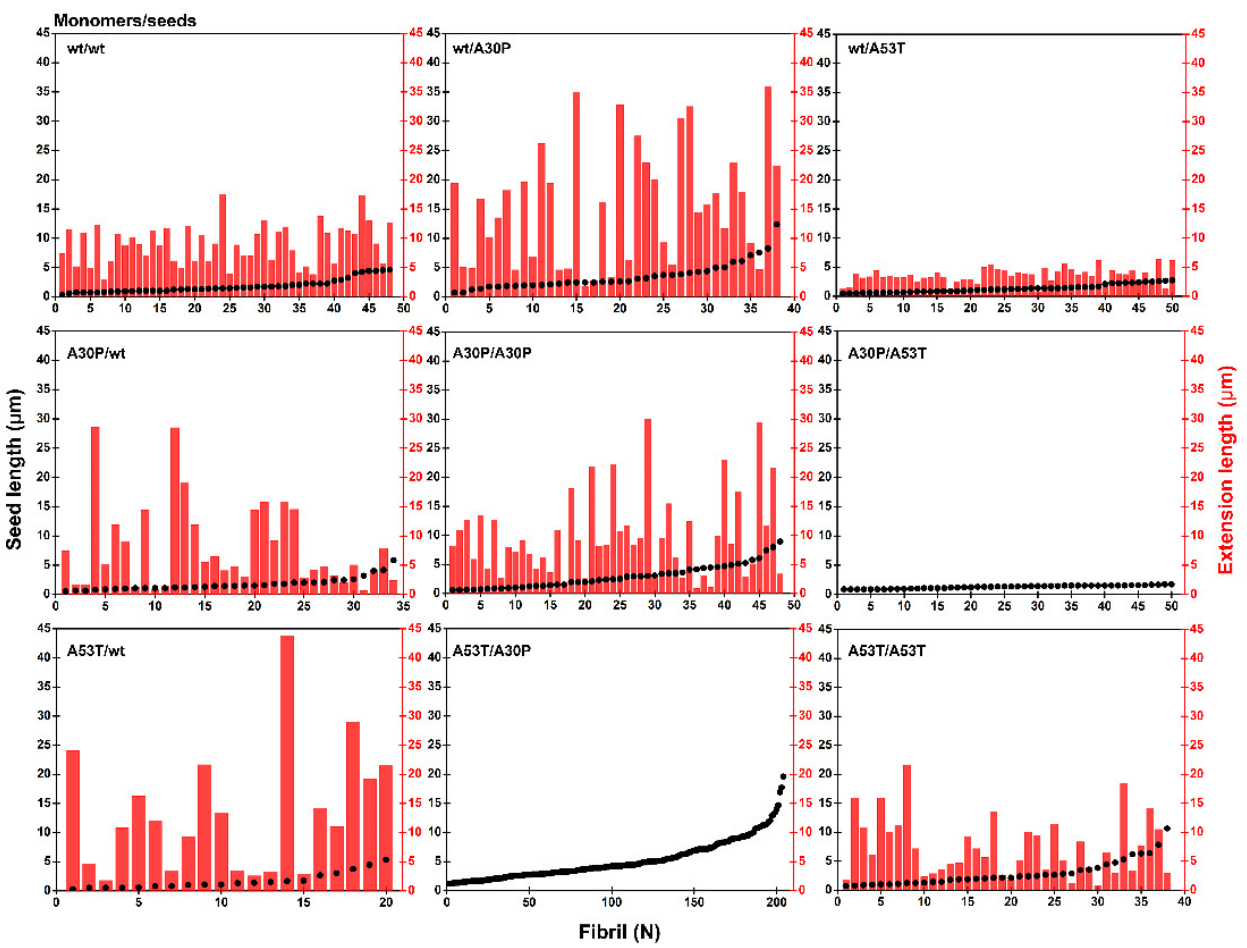

Figure 5.7 Histograms showing elongation per seed in different seeded aggregation reactions (the extension length is only for the longer extension in case of bidirectional growth). 


\section{Directionality of seed extension is seed specific}

An additional aspect of seed elongation is the elongation rate at the two sides of the seeds. The elongation rate of a seed is believed to depend on the ratio of molecular association and dissociation at the fibril ends (Carulla, Caddy et al. 2005). Since the two ends are formed of the same protein unit, one could reasonably expect each end to be equivalent. However, several studies on amyloids propose asymmetric seed elongation (Goldsbury, Kistler et al. 1999; Scheibel, Kowal et al. 2001; Ban, Hoshino et al. 2004; Heldt, Zhang et al. 2011; Pinotsi, Buell et al. 2014). In our study, a comparison between the different types of seeds shows that the aggregations with wt and A30P seeds primarily elongate in a unidirectional manner. The fibrillizations with A53T seeds in contrast showed about $50 \%$ unidirectional and $50 \%$ bidirectional fibril growth (Figure 5.8). However, the length of the two extensions on all of the seeds exhibiting bidirectional growth was not similar, i.e. one side was always longer than the other. The inequality in fibril elongation at the two ends of a seed points towards different rate of elongation on the two sides. In all, the growth patterns observed among the different seeds indicates that the type of the seed governs the symmetry of seed elongation and that the two ends of the seeds are not equivalent.
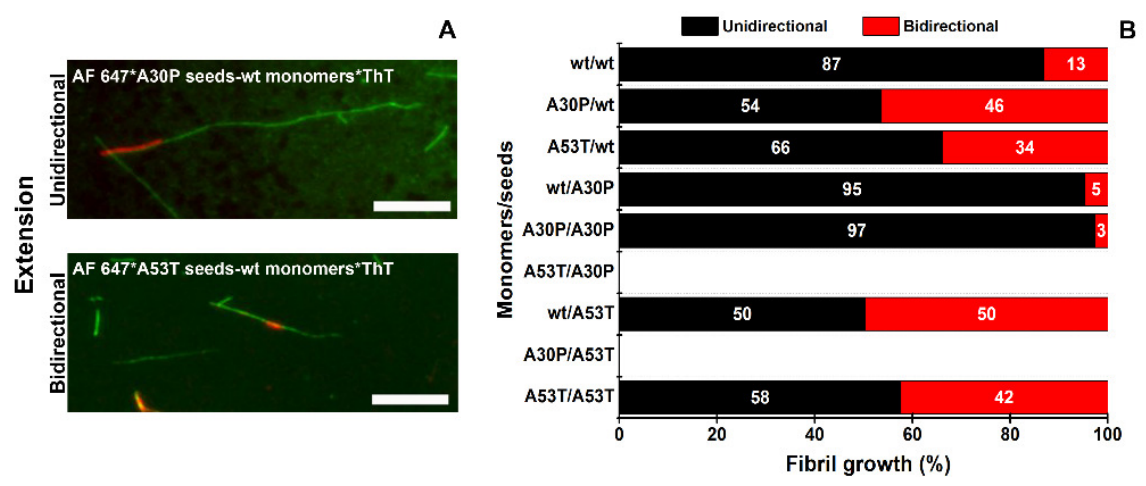

Figure 5.8 (A) Representative widefield fluorescence images showing unidirectional and bidirectional elongation of seeds. Seeds were labeled with AF 647 and the extensions were visualized by ex situ binding of ThT. Scale bar: $5 \mu \mathrm{m}$. (B) Histogram showing proportion of unidirectional and bidirectional growth in different seeded aggregations. 


\section{CONCLUSIONS}

Overall, the results show that in aSyn seeded aggregation reactions, in the given solution conditions, conformational compatibility between the seed and the soluble monomer is important for seed elongation. Although $\alpha$ Syn is characterized as an intrinsically disordered protein, the monomers likely exist in dynamic ensembles in a collapsed coil state (with long range intramolecular interactions) (Dedmon, Lindorff-Larsen et al. 2005; Lee, Lai et al. 2007). The distribution of the ensembles is critically influenced by the solution conditions. In addition, a single amino acid difference in the protein sequence can lead to pronounced variation in the thermodynamically favorable conformations (Chiti, Stefani et al. 2003). Therefore, in a given solution condition minor variations in the polypeptide sequence can result in fibrils of distinct morphology. While heterologous aggregations between seeds and monomers of conflicting conformation deter elongation of seeds, in the event of cross seeding the aggregation characteristics are determined by the properties of the seeds. The observed conformational stringency for seed elongation may have implications for in vivo propagation of amyloids and strain selection, as conformation and not sequence appears to be key for inducing aggregation in soluble $\alpha$ Syn.

\section{ACKNOWLEDGEMENTS}

We thank Kirsten van Leijenhorst-Groener, Yvonne Kraan and Nathalie Schilderink for protein expression and purification. 


\title{
Chapter 6
}

\section{Functionalizing $\alpha$-synuclein amyloid fibrils to create self-assembled bionanostructures}

\begin{abstract}
Amyloid fibrils are promising candidates for the bottom up fabrication of nano- to mesoscopic assemblies owing to their nanoscale organization and excellent material properties. Amyloid fibrils formed by aSyn protein are characterized by a typical diameter of about $7 \mathrm{~nm}$ and lengths in microns. Site-specific incorporation of a cysteine residue enables a wide range of secondary modifications, providing a molecular 'hook' which can be used for chemical modifications with different functional groups or even nanoparticles. In this chapter, we explore the functionalization of aSyn fibrils with gold nanoparticles to prepare transmission competent bio-nanostructures. To do so, we substitute the C-terminal alanine residue by a cysteine (A140C) and use the fibril assembly conditions optimized in chapter 2 to prepare morphologically homogeneous fibrils. We functionalize these fibrils at the cysteine residues with maleimide conjugated $1.4 \mathrm{~nm}$ or $5 \mathrm{~nm}$ diameter colloidal gold nanoparticles. To make these gold nanoparticlelabeled fibrils conductive, gold enhancement methods were optimized resulting in a dense arrangement of gold nanoparticles on the amyloid scaffold that exhibits high potential for conductive nanowires.
\end{abstract}




\section{INTRODUCTION}

Miniaturization of technology to ever-smaller scales necessitates the use of bottom up fabrication methods to produce the required nano- to microscopic components. Biomolecules like DNA and proteins are suitable for bottom up fabrication due to their inherent nanoscopic size and their ability to self-assemble into well-defined larger structures given the optimal assembly conditions (Mann 2009). The resultant biomolecular assemblies can be specifically functionalized with a range of functional elements like metallic nanoparticles, quantum dots and fluorophores to impart the desired function.

Gold nanoparticles (AuNPs) are the most commonly used particles for functionalization. AuNPs are conductive and can be used to fabricate nanowires, as demonstrated by nano-gold metalized DNA and protein assemblies (Liu, Meyer-Zaika et al. 2003; Scheibel, Parthasarathy et al. 2003; Yan, Park et al. 2003; Bai, Xu et al. 2007; Sarangi, Goswami et al. 2007; Bjork, Herland et al. 2010; Ostrov and Gazit 2010). In addition, AuNPs exhibit unique size and shape dependent physico-chemical properties like localized surface plasmon resonance that has enabled development of nano-sensors (Yan, Park et al. 2003; Patolsky, Weizmann et al. 2004; Rosi and Mirkin 2005; Doria, Conde et al. 2012). Bulk gold is an inert metal and has been used in surgical implants in the past. However, the properties of nano-gold are different from those of bulk gold; as a consequence, long term biological safety of nano-gold is still under investigation (refer to appendix A for detailed risk assessment of AuNPs used in the present study) (De Jong, Hagens et al. 2008; Dreaden, Alkilany et al. 2012). Although both protein- and DNA-based self-assembled bionanostructures have been explored for functionalization, in the present chapter we focus on the AuNP functionalization of fibrillar protein assemblies with an aim to fabricate conductive and photonic bionanostructures.

Amyloids as material: A number of proteins and peptides are known to selfassemble into amyloids in in vitro conditions (Dobson 2003). The monomeric amyloidogenic proteins self-assemble into amyloid fibrils spontaneously under optimized solution conditions, usually giving rise to structures of 7-10 nm in diameter but with variable lengths. Besides the pathological amyloids, some of the amyloids found in nature, named functional amyloids, are associated with definite function to 
the parent organism, for example, the protective shell formed by egg shell chorion protein by silkmoth (Hamodrakas, Hoenger et al. 2004), the hyphae ultrastructure formed by chaplins, assisting in spore dispersal, in Streptomyces coelicor (Capstick, Jomaa et al. 2011) and analogous fungal proteins, hydrophobins, that aid in withstanding the surface tension at the air-water interface (Elliot and Talbot 2004). These examples from nature demonstrate some of the excellent and unique material properties of amyloids. In vitro experiments with a range of natural and synthetic selfassembling protein sequences have shown amyloids to have high tensile strength and resistance to high pressure and temperature (up to $500 \mathrm{MPa}$ and $100{ }^{\circ} \mathrm{C}$, respectively) (Meersman and Dobson 2006; Smith, Knowles et al. 2006). Furthermore, amyloids are proposed to be decidedly stable in the biological milieu, being inert to action of proteases and changes in $\mathrm{pH}$ (Chiti and Dobson 2006). Owing to these valuable material properties, self-assembled amyloids seem ideally suited for bionanomaterials like scaffolds for nanowires, matrices for 3D cell cultures and nano-composite materials. However, the safety of amyloids, particularly those associated with diseases, is being increasingly questioned with the emerging evidence for prion-like behavior of amyloids.

Different functional groups on the protein molecules like the amino $\left(-\mathrm{NH}_{2}\right)$, carboxyl (-COOH) and the sulfhydryl (-SH) groups enable chemical modifications to impart novel functional properties to the protein and the resultant amyloid fibrils. Conjugation with metallic nanoparticles and polyelectrolytes in the past has produced conductive and optically active nanowires (chapter 1, Table 1.2). Further, coprecipitation of amyloids with inorganic materials like fluorophores, gold, graphene and hydroxyapatite result in composite materials with physical and functional properties of both the components (Knowles, Oppenheim et al. 2010; Li, Adamcik et al. 2012; Li, Bolisetty et al. 2013; Li, Born et al. 2014; Zhou, Saha et al. 2015).

aSyn amyloids as scaffolds: Here, we study the potential of aSyn amyloids as scaffolds for the preparation of transmission competent assemblies. Fibrillization of aSyn monomers typically results in polymorphic fibrils (Heise, Hoyer et al. 2005; Vilar, Chou et al. 2008). Polymorphism can be a deterrent for use of fibrils as scaffold since fibrils of different morphology are suggested to have different mechanical properties (Yoon, Lee et al. 2014). In chapter 2, we showed that depending on the aggregation conditions, aSyn monomers could be induced to fibrillize into near homogeneous 
fibrils at the plateau phase. Using the solution conditions optimized in chapter 2, we prepared fibrils from an aSyn mutant carrying a cysteine at position 140 instead of an alanine (A140C) and explored the functionalization of these fibrils with 1.4 and $5 \mathrm{~nm}$ AuNPs to fabricate conductive nanowires. We find that the aSyn fibrils can be successfully functionalized with 1.4 and $5 \mathrm{~nm}$ AuNPs. After AuNP functionalization the density of the gold on the fibril surface can be increased appreciably using electroless gold enhancement leading to formation of promising transmission competent assemblies.

\section{MATERIALS AND METHODS}

\section{$\alpha$ Syn expression and purification}

Wt and A140C aSyn protein were used in the experiments, and the proteins were prepared, expressed and purified as described elsewhere (Sidhu, Segers-Nolten et al. 2014).

\section{Labeling of A140C $\alpha$ Syn monomers with $1.4 \mathrm{~nm}$ AuNPs}

$250 \mu \mathrm{l}$ monomeric stock of A140C aSyn stored at $-80^{\circ} \mathrm{C}$ was thawed and reduced with freshly prepared $1 \mathrm{mM}$ dithiothreitol (DTT) solution at room temperature for 1 hour. The protein solution was desalted using $2 \mathrm{ml}$ Zeba spin columns $(7 \mathrm{k}$ MWCO, Pierce Biotechnology) following the manufacturer's protocol to remove DTT and the buffer was changed to $0.1 \mathrm{M}$ sodium phosphate, $150 \mathrm{mM} \mathrm{NaCl}$ and $1 \mathrm{mM}$ EDTA at pH 6.5. The concentration of the reduced protein was determined by measuring the absorbance at $280 \mathrm{~nm}$ on a NanoDrop ND-1000 spectrophotometer (Isogen Life Science). Then, 6 nmoles of Monomaleimido Nanogold ${ }^{\odot}$ Labeling Reagent (Nanoprobes, Cat\# 2020A), stored at $-20^{\circ} \mathrm{C}$ was reconstituted in $0.9 \mathrm{ml}$ of Milli- $\mathrm{Q}$ water. The dissolved reagent was immediately mixed with 6 nmoles of reduced protein monomer solution $(0.1 \mathrm{ml})$ and incubated at room temperature for 2 hours with gentle mixing. After incubation, the unreacted gold particles were removed using two $2 \mathrm{ml}$ Zeba spin desalting columns sequentially. The stoichiometry of labeling of the purified protein sample was determined by measuring the absorbance of the protein $\left(\mathrm{A}_{280 \mathrm{~nm}}\right)$ and AuNPs $\left(\mathrm{A}_{420} \mathrm{~nm}\right)$ and, using extinction coefficients of 5745 and $155,000 \mathrm{M}^{-1} \mathrm{~cm}^{-1}$, respectively. 


\section{Preparation of $\alpha$ Syn fibrils from 1.4 nm AuNPs labeled monomers}

Fibrils were prepared using 5, 25 and $50 \mu \mathrm{M}$ AuNPs labeled A140C monomers, and 95, 75 and $50 \mu \mathrm{M}$ unlabeled wt monomers respectively in $10 \mathrm{mM}$ Tris- $\mathrm{HCl}, 10 \mathrm{mM} \mathrm{NaCl}$ and $0.1 \mathrm{mM}$ EDTA at pH 7.4. The aggregation was followed by ThT assay as described earlier (Sidhu, Segers-Nolten et al. 2014).

\section{Labeling of A140C $\alpha$ Syn fibrils with $1.4 \mathrm{~nm}$ AuNPs}

Fibrils prepared from $100 \mu \mathrm{M}$ A140C monomers in $10 \mathrm{mM}$ Tris- $\mathrm{HCl}, 10 \mathrm{mM} \mathrm{NaCl}, 0.1$ mM EDTA, 2 mM DTT at pH 7.4 (as described earlier (Sidhu, Segers-Nolten et al. 2014)) were labeled with $1.4 \mathrm{~nm}$ AuNPs in solution as well as on a substrate. Labeling of the fibrils in solution aimed at fabricating three dimensionally functionalized aSyn fibrils, which could be deposited on a desired substrate later. Fibrils were labeled on a substrate to troubleshoot the poor adsorption of the labeled fibrils on a silicon based substrate, the clustering of fibrils, and the inefficiency of the gold enhancement step, encountered while labeling in solution.

\section{Labeling in solution}

$100 \mu \mathrm{l}$ of the plateau phase A140C fibril sample $(100 \mu \mathrm{M})$ was centrifuged at $21,000 \times \mathrm{g}$ for 1 hour at room temperature. The supernatant was aspirated to remove the monomers and the pellet was resuspended in $0.8 \mathrm{ml}$ of $0.1 \mathrm{M}$ sodium phosphate, 150 $\mathrm{mM} \mathrm{NaCl}$ and $1 \mathrm{mM}$ EDTA at $\mathrm{pH}$ 6.5. 6 nmoles of Monomaleimido Nanogold Labeling Reagent (Nanoprobes, Cat\# 2020A), resuspended in $0.2 \mathrm{ml}$ of Milli-Q water was added to the fibrils and incubated for 2 hours at room temperature or overnight at $4{ }^{\circ} \mathrm{C}$ with gentle mixing. After incubation, the sample was centrifuged at $8000 \times \mathrm{g}$ for 20 minutes. The supernatant was discarded while the labeled fibrils in the pellet were resuspended in $0.5 \mathrm{ml}$ of $10 \mathrm{mM}$ Tris- $\mathrm{HCl}, 10 \mathrm{mM} \mathrm{NaCl}$ at $\mathrm{pH} 7.4$.

\section{Labeling on a substrate}

For labeling the fibrils on a substrate, two substrates were tested; the TEM grid, to assess the protocol of labeling and gold enhancement through TEM and STEM; and mica, to perform conductive probe AFM experiments, to measure the conductive properties of the functionalized fibrils. TEM grids used (CF200-Cu-UL, EMS) were square meshed 
copper grids with a thin film of carbon of 3-4 $\mathrm{nm}$ thickness on one side. For TEM grids as well as mica, same protocol was followed for labeling and electroless gold enhancement (see next section). Fibril preparations from the plateau phase were diluted 10 to 15 fold in $10 \mathrm{mM}$ Tris- $\mathrm{HCl}, 10 \mathrm{mM} \mathrm{NaCl}$ at $\mathrm{pH} 7.4$ and deposited on a clean TEM grid $(5 \mu \mathrm{l})$ or freshly cleaved mica (10 $\mu$ l, Muscovite, V-1 quality, EMS). The sample was adsorbed for 20 minutes, followed by 2 gentle washes with $100 \mu \mathrm{l}$ of fresh Milli-Q water. $5 \mu \mathrm{l}$ (TEM grid) and $20 \mu \mathrm{l}$ (mica) of 6 nmoles of Monomaleimido Nanogold ${ }^{\oplus}$ Labeling Reagent (Nanoprobes, Cat\# 2020A), resuspended in $1 \mathrm{ml}$ of Milli-Q water, was added to the fibrils and incubated for 1 hour at room temperature. After incubation, the fibrils were washed with $200 \mu \mathrm{l}$ of Milli-Q water to remove unreacted AuNPs and was allowed to dry in air at room temperature.

\section{Electroless gold enhancement}

In solution

The size of the AuNPs was enlarged using a Gold Enhancement with GoldEnhance ${ }^{\text {mx }}$ EM kit from Nanoprobes. The kit solutions are propriety solutions and the enhancement protocol used was based on manufacturer's recommendations. Solutions stored at $4{ }^{\circ} \mathrm{C}$ were brought to room temperature and $5 \mu \mathrm{l}$ of solutions $\mathrm{A}$ and $\mathrm{B}$ were mixed (per reaction) by vortexing 5 minutes prior to use. Just before use $5 \mu$ l of solution $\mathrm{C}$ and water (instead of solution $\mathrm{D}$ to reduce the rate of reduction of the gold ions and prevent autonucleation) were mixed and applied to $0.5 \mathrm{ml}$ of nanogold labeled fibrils (in $10 \mathrm{mM}$ Tris- $\mathrm{HCl}, 10 \mathrm{mM} \mathrm{NaCl}$ at $\mathrm{pH}$ 7.4) for 2 to 5 minutes at room temperature. To stop the reaction the samples were centrifuged at 10,000 $\times \mathrm{g}$ for 20 minutes at room temperature. The supernatant was discarded and the pellet was washed with $0.5 \mathrm{ml}$ Milli-Q water, centrifuged again and the resulting pellet was resuspended in $0.5 \mathrm{ml}$ of $10 \mathrm{mM}$ Tris- $\mathrm{HCl}, 10 \mathrm{mM} \mathrm{NaCl}$ at $\mathrm{pH}$ 7.4.

\section{On a substrate}

Electroless gold enhancement of AuNP labeled fibrils on a substrate was done in two steps at $60{ }^{\circ} \mathrm{C}$. The reagents for electroless gold enhancement were prepared as mentioned in the previous section. For the first step, $5 \mu \mathrm{l}$ (TEM grid) and $20 \mu \mathrm{l}$ (mica) of a 2 fold diluted enhancing solution was applied to the adsorbed fibrils as a primer for 2 minutes. The samples were washed twice with $50 \mu \mathrm{l}$ Milli-Q water. In the second step, 
$5 \mu \mathrm{l}$ (TEM grid) and $50 \mu \mathrm{l}$ (mica) of the enhancement solution was applied to the fibril samples for 2, 3 and 5 minutes. After incubation, the samples were washed twice with $100 \mu \mathrm{l}$ Milli-Q water and the samples were kept to dry in air at room temperature.

\section{Preparation of $5 \mathrm{~nm}$ AuNPs labeled $\alpha$ Syn fibrils during fibrillization}

To prepare fibrils labeled with $5 \mathrm{~nm}$ colloidal particles a passive approach of coincubating the aggregation reaction with gold particles was tested. $150 \mu \mathrm{l}$ of $5 \mathrm{~nm}$ colloidal AuNPs stabilized in citrate buffer (Aldrich; concentration $\approx 5.5 \times 10^{13}$ particles $/ \mathrm{ml}$ ) were added to an aggregation reaction of $100 \mu \mathrm{M}$ A140C aSyn, $10 \mathrm{mM}$ Tris- $\mathrm{HCl}, 10 \mathrm{mM} \mathrm{NaCl}, 2 \mathrm{mM}$ DTT and 0.1 mM EDTA at $\mathrm{pH}$ 7.4.

\section{Labeling of A140C $\alpha$ Syn fibrils with $5 \mathrm{~nm}$ colloidal AuNPs Labeling in solution}

Fibrils assembled from A140C monomers (as described earlier (Sidhu, Segers-Nolten et al. 2014)) were dialyzed against $10 \mathrm{mM} \mathrm{NaCl}$ overnight at $4{ }^{\circ} \mathrm{C}$ to exchange Tris- $\mathrm{HCl}$ buffer. The sample was buffered with $10 \mathrm{mM}$ sodium acetate solution at $\mathrm{pH} 3.6$ and allowed to equilibrate at room temperature for 1 hour. Next, $150 \mu \mathrm{l}$ of $5 \mathrm{~nm}$ colloidal AuNPs stabilized in citrate buffer (Aldrich; concentration $\approx 5.5 \times 10^{13}$ particles $/ \mathrm{ml}$ ) were added to $0.5 \mathrm{ml}$ fibril sample ( 15 nmoles of equivalent monomeric mass) and incubated at room temperature for 2 hours with gentle mixing.

\section{Labeling on a substrate}

A140C fibrils were adsorbed on a TEM grid or freshly cleaved mica and washed with $100 \mu \mathrm{l}$ of fresh Milli-Q water. Next, the fibrils were equilibrated with 5 (TEM grid) or 20 (mica) $\mu \mathrm{l}$ of $10 \mathrm{mM}$ sodium acetate solution at $\mathrm{pH} 3.6$ for 20 minutes at room temperature. Then, $5 \mu \mathrm{l}$ (TEM grid) or $10 \mu \mathrm{l}$ (mica) of $5 \mathrm{~nm}$ colloidal gold particles were added to the fibril sample for 20 minutes. The samples were washed twice with $50 \mu \mathrm{l}$ of Milli-Q water and allowed to dry in air.

\section{Labeling of A140C $\alpha$ Syn fibrils with $5 \mathrm{~nm}$ and $1.4 \mathrm{~nm}$ AuNPs}

For preparation of fibrils labeled with both 5 and $1.4 \mathrm{~nm}$ AuNPs, labeling with $5 \mathrm{~nm}$ AuNPs was followed by labeling with $1.4 \mathrm{~nm}$ AuNPs as described above. 


\section{Atomic force microscopy (AFM)}

Functionalized and unlabeled control samples were imaged with AFM in tapping mode in air. The samples were prepared by adsorbing fibrils on freshly cleaved mica (Muscovite, V-1 quality, EMS) for 4 minutes, followed by washing with $100 \mu$ l of Milli$\mathrm{Q}$ water and gently drying in a stream of nitrogen gas (filtered through a $0.22 \mu \mathrm{m}$ filter, Millipore). Images were acquired on a Bioscope Catalyst (Bruker) in soft tapping mode using NSC36 cantilever, tip B. The images were analyzed using Scanning Probe Image Processor-6.0.13 (SPIP-Image Metrology).

\section{Sample preparation for electron microscopy (EM)}

Samples for EM were prepared on a TEM copper grid with ultrathin carbon film (CF200-Cu-UL, EMS). For labeled samples, TEM samples were prepared as described above. For unlabeled control samples, $100 \mu \mathrm{M}$ aggregation mix was diluted 10 fold. $5 \mu \mathrm{l}$ of the diluted sample was placed on the grid and allowed to adsorb for 5 minutes at room temperature. Then the sample was washed with $200 \mu \mathrm{l}$ of Milli-Q water. Following the sample was negatively stained by $2 \%$ uranyl acetate for 30 seconds, washed with 200 $\mu \mathrm{l}$ of Milli-Q water and the grid was allowed to air dry. For STEM the control samples were not negatively stained.

\section{Transmission electron microscopy (TEM) and energy-dispersive X-ray spectroscopy (EDX-analysis)}

The samples were imaged on a CM300ST-FEG TEM (Philips) installed at the MESA+ NanoLab, University of Twente. The TEM $(300 \mathrm{kV})$ system is equipped with a GATAN Tridiem energy filter $(2 \mathrm{k} \times 2 \mathrm{k}$ CCD camera), extra GATAN Ultrascan 1000 CCD camera $(2 \mathrm{k} \times 2 \mathrm{k})$, and Noran System Six EDX analyzer.

\section{Scanning transmission electron microscopy (STEM)}

Samples were imaged in STEM mode on a Verios ${ }^{\text {tm }}$ XHR SEM instrument (FEI). The samples were imaged with a STEM III detector using an electron beam of 20 to $30 \mathrm{kV}$ and a probe current of 6.3 to $50 \mathrm{pA}$ in the lens immersion mode. The images were acquired over a dwell time of $30 \mu$ s with $1536 \times 1024$ pixels. 


\section{Measuring conductive properties of functionalized fibrils}

Micro-interdigitated electrodes (microIDTs)

Experiment design: The experiment was designed with the view that a network of gold labeled fibrils when drop casted on an array of micron spaced electrodes and subjected to a bias voltage should result in flow of a current, if the functionalized fibrils are conductive (Figure 6.1A).

Sample preparation and measurement: Interdigitated electrodes with $2.5 \mu \mathrm{m}$ gap (microIDTs) between the electrodes were fabricated on a silicon wafer in the clean room. The fabricated microIDTs were inspected with a dark field microscope (Olympus GX71) to check for fabrication defect of fused electrodes. Next, the fibril sample was deposited on the microIDTs by drop casting. $5 \mu$ of the sample was applied on the microIDTs and allowed to adsorb for 20 minutes. Then the sample was washed twice with $50 \mu \mathrm{l}$ of Milli-Q water and air dried. A recheck of the microIDTs in the dark field microscope showed very poor sample deposition. Nevertheless, the conductivity of the sample was checked using a probe station with a bias voltage of 1,5 and $20 \mathrm{~V}$.

\section{Conductive probe AFM}

Experiment design: The conductive properties of the gold-functionalized fibrils were investigated by conductive probe AFM (schematically outlined in Figure 6.1B). A mica surface with adsorbed gold labeled fibrils was partially sputtered with a gold layer by shadow masking to create gold masked and unmasked sections. One electrode from the conductive probe AFM was clamped on the sputtered gold layer. A conductive tip, as a second electrode, was scanned across the fibrils at the gold/mica interface. In this way the presence of conductive, gold functionalized fibrils could be mapped.

Sample preparation: A140C fibrils were deposited on a mica surface and were labeled with AuNPs. The mica surface was partially sputtered with a gold layer of about $30 \mathrm{~nm}$ thickness using a custom built film sputtering setup, Sputterke, in the clean room, with a deposition rate of about 45-50 $\mathrm{nm} \mathrm{s}^{-1}$. To realize a razor-edge gold-mica interface, small pieces of silicon were utilized as mask on the mica surface. 


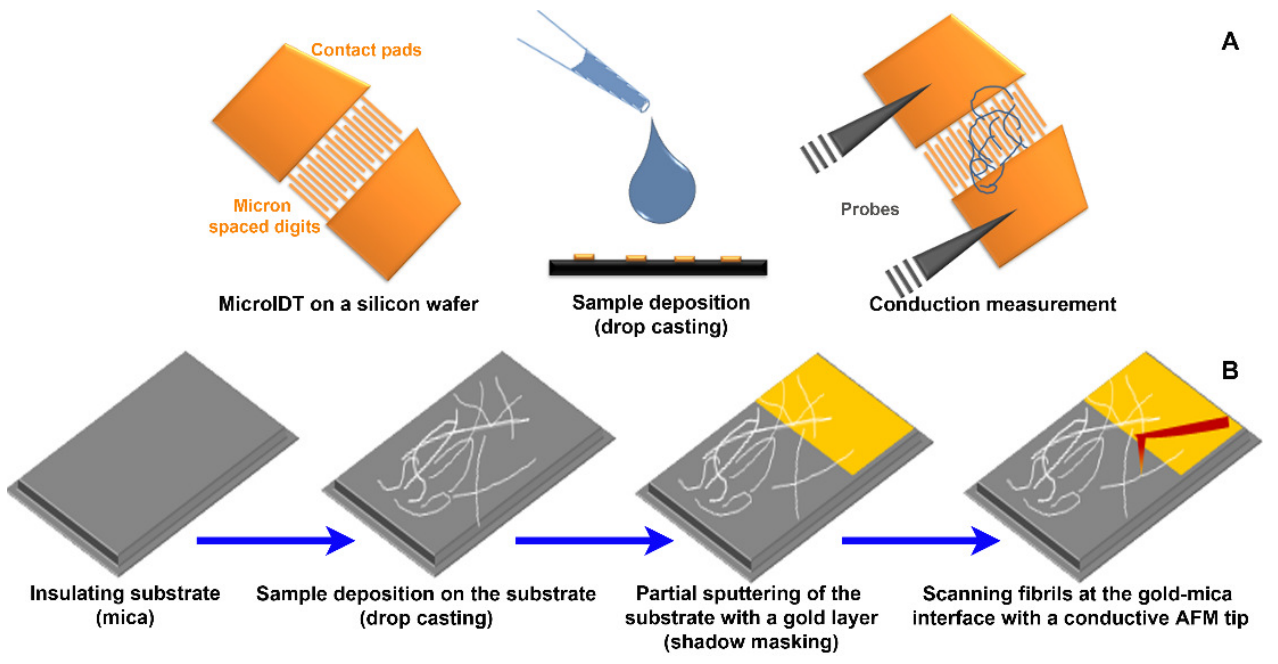

Figure 6.1 Schematic representation of the experiments to measure conductive properties of the AuNPs functionalized fibrils (A) by microIDTs and (B) by conductive probe AFM.

\section{Conductive probe AFM imaging}

The conductive properties of the samples were imaged by conductive probe AFM using a Bruker AFM Dimension ICON in Peak force TUNA mode, with a DC bias voltage of $20 \mathrm{mV}$. The samples were scanned with a silicon nitride cantilever with a PlatinumIridium coating (Bruker PFTUNA), with a force constant of $0.4 \mathrm{~N} / \mathrm{m}$. The AFM images were analyzed with Scanning Probe Image Processor-6.0.13 (SPIP) (Image Metrology) and overlaid with ImageJ.

\section{RESULTS AND DISCUSSION}

\section{Functionalization of $\alpha$ Syn fibrils with AuNPs in solution}

aSyn mutant containing cysteine at position 140 was prepared using site directed mutagenesis. The C-terminal residue of the protein, alanine, was substituted by a cysteine, as the - $\mathrm{SH}$ group on the cysteine molecule facilitates biochemical modifications involving thiol-maleimide chemistry (Figure 6.2). In addition, the Cterminus of the protein is not known to be included in the fibril core but is suggested to be on the fibril surface, i.e. accessible to the solution, thus allowing modifications on the 
assembled fibrils (Del Mar, Greenbaum et al. 2005; Qin, Hu et al. 2007; Guilliams, ElTurk et al. 2013).

Two types of AuNPs were used to functionalize the fibrils, $1.4 \mathrm{~nm}$ mono-maleimide conjugated gold nanoparticles (1.4 nm AuNPs) and $5 \mathrm{~nm}$ colloidal gold particles. For incorporation of $1.4 \mathrm{~nm}$ AuNPs on the fibril surface two approaches were tested, through fibrillization of A140C monomers labeled with AuNPs or through conjugation of AuNPs after assembly of the fibrils. Labeling with $5 \mathrm{~nm}$ colloidal AuNPs was tried by including the particles in an aggregation reaction and by adding them to assembled fibrils.

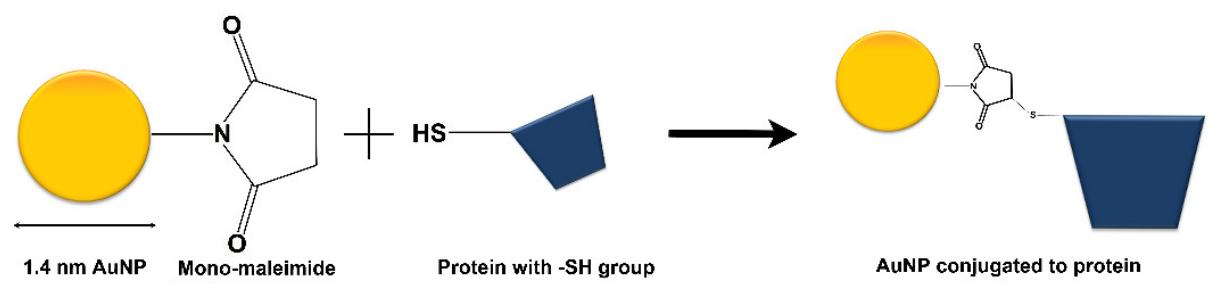

Figure 6.2 Scheme demonstrating conjugation of gold nanoparticles linked with a maleimide group to a cysteine carrying protein molecule, through thiol-maleimide chemistry.

\section{Labeling with $1.4 \mathrm{~nm}$ AuNPs in solution}

\section{Labeling by incorporation of $1.4 \mathrm{~nm}$ AuNP labeled monomers during fibrillization}

In the first approach, 6 nmoles of A140C monomers were labeled with 6 nmoles of 1.4 nm AuNPs. The labeled monomers were aggregated with wt unlabeled monomers in 1:20, 1:4 and 1:1 ratios (labeled to unlabeled ratios). The fibrillization followed by ThT assay showed an increase in fluorescence in reactions with 1:20 monomers (i.e. $1.4 \mathrm{~nm}$ AuNPs labeled A140C to unlabeled wt) followed by reaction with 1:4 labeled to unlabeled monomers, while reaction with 1:1 labeled monomers did not show any increase in ThT fluorescence (Figure 6.3A). AFM imaging of the 1:1 labeled sample did not show typical amyloid fibrils but amorphous elongated features of about $1.5 \mathrm{~nm}$ height (Figure 6.3B and C). Based on AFM images it was difficult to comment on the nature of these assemblies. For the other monomer mixtures, fibrils from the ThT plateau phase were imaged by TEM to assess labeling by the AuNPs. The sample with 1:20 labeling ratio showed very few gold particles on the fibrils while the sample with 


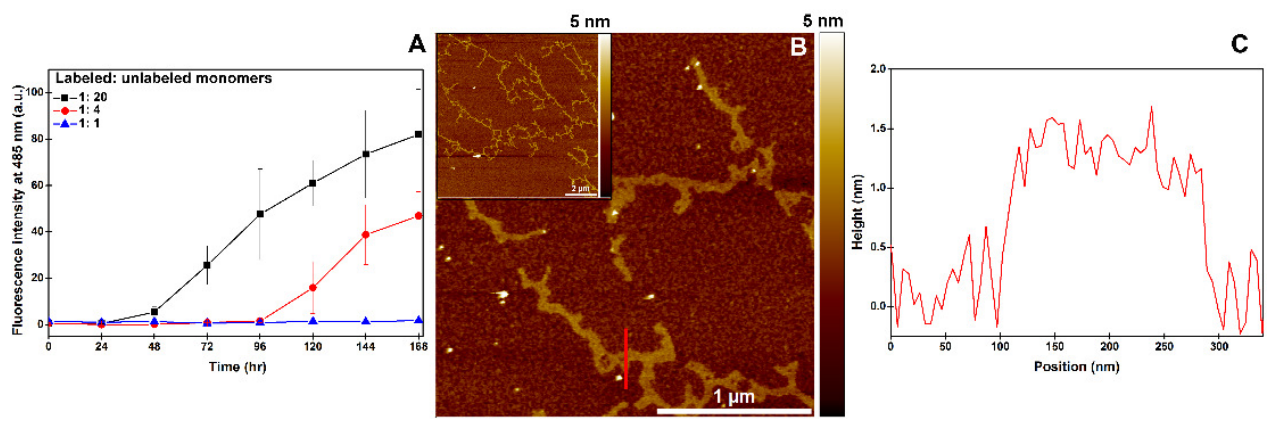

Figure 6.3 (A) ThT assay showing fibrillization kinetics of aggregation reactions with $1.4 \mathrm{~nm}$ AuNPs labeled A140C and unlabeled wt aSyn monomers in the ratios of 1:20, 1:4 and 1:1. (B) Representative AFM height image of aggregates produced in 1:1 labeled reaction. Inset: overview image (C) Height profile of the aggregates in " $\mathrm{B}$ " at the position indicated by the red line.

1:4 labeling ratio had a comparatively higher density of gold particles (Figure 6.4A, B and $\mathrm{C}$ ). The presence of gold particles on the fibrils was confirmed by energy-dispersive X-ray spectroscopy (EDX) analysis on a small cross section of the fibrils. The EDX spectra showed peaks for carbon, copper, oxygen and gold (Figure 6.4D). The source of gold on the sample is in all likelihood the gold particles used for functionalization while carbon and copper were part of the TEM grid used for the sample deposition. Although it seems in principle possible to functionalize the fibrils with AuNPs using 1:20 and 1:4 labeling ratios, the density of gold particles that followed from this approach was evidently too low to impart conductive properties to the functionalized fibrils.

Both ThT fluorescence experiments and AFM images suggest that using high labeled to unlabeled monomer ratios (like 1:1) does not favor assembly of the monomers into fibrils. In addition to the steric hindrance, the labeled monomer may also experience an altered electrostatic environment due to the surface chemistry of the AuNPs. AuNPs are routinely stabilized via surface functionalization of organic, hydrophilic or hydrophobic molecules to maintain single particle suspensions (Sperling and Parak 2010). Such modifications may hamper intermolecular interactions necessary for protein fibrillization when present at high concentrations, hence explaining the absence of fibrillar assemblies in aggregation with 1:1 labeled to unlabeled reactions. 

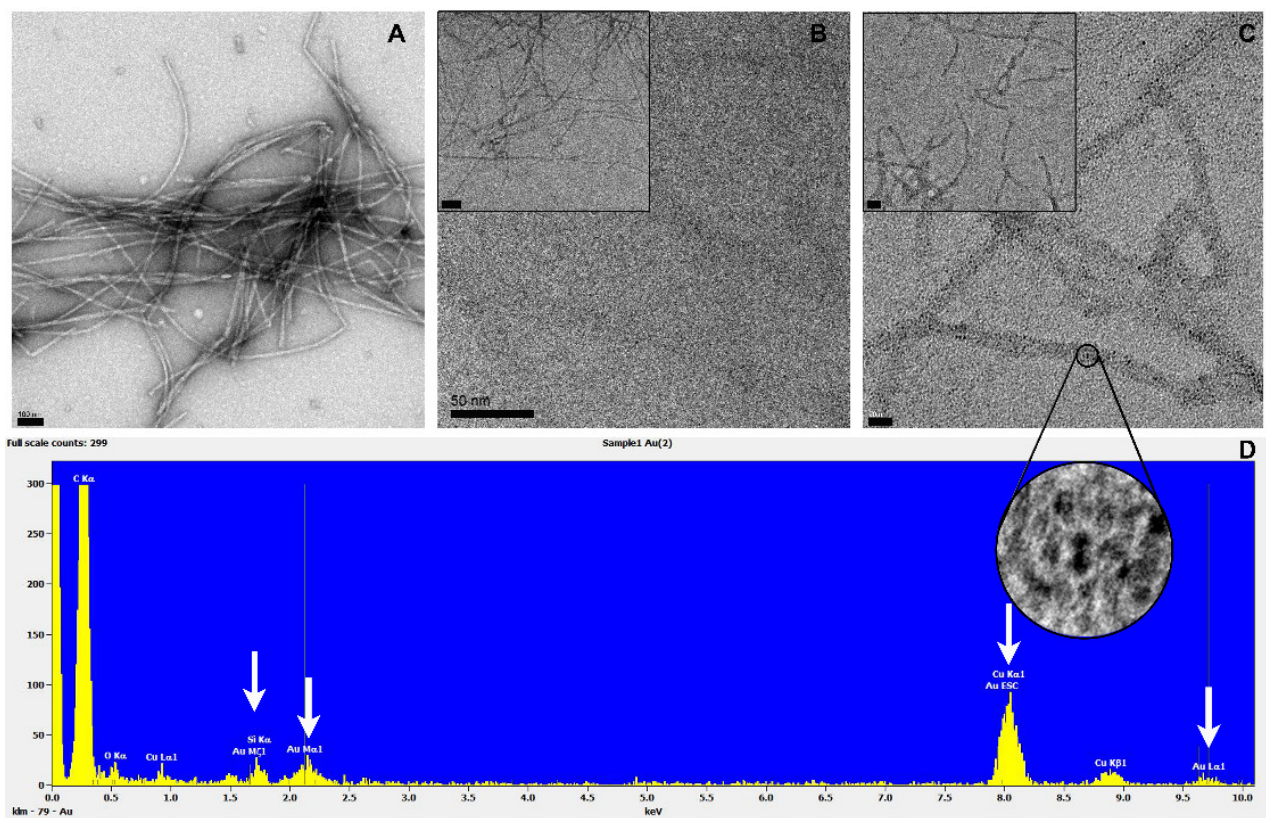

Samplet Au(2)

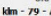

Figure 6.4 Representative TEM images of aSyn fibrils. (A) Unlabeled A140C aSyn fibrils, stained with $2 \%$ uranyl acetate solution, scale bar: $100 \mathrm{~nm}$ (B) Fibrils prepared from $1.4 \mathrm{~nm}$ AuNPs labeled monomers and unlabeled wt monomers in 1:20 ratio, not stained, scale bar: $50 \mathrm{~nm}$. Inset: overview image, scale bar: $200 \mathrm{~nm}$. (C) Fibril prepared with 1:4 (labeled to unlabeled) monomers, not stained, scale bar: $20 \mathrm{~nm}$. Inset: overview image, scale bar: $50 \mathrm{~nm}$. (D) EDX analysis on a small section of TEM image $\mathrm{C}$ showing the presence of elemental gold (marked by white arrows).

\section{Labeling of assembled fibrils}

In the second approach, to avoid possible interference in the self-assembly of aSyn due to AuNPs, we self-assembled A140C monomers into fibrils and then labeled the resultant fibrils with $1.4 \mathrm{~nm}$ AuNPs. Labeling the fibrils as opposed to monomers considerably improved the gold particle density on the fibrils. The average center to center interparticle distance between the AuNPs on the fibrils was $3.3 \pm 1.7 \mathrm{~nm}$ (Figure 6.5A and B). To reduce the interparticle distance further, the $1.4 \mathrm{~nm}$ AuNPs labeled fibrils were exposed to electroless gold enhancement for 2 to 5 minutes. The enhancement process however was inefficient as only a few gold particles conjugated to the fibrils increased in size (Figure 6.5C). Moreover, appreciable nucleation of the gold particles could be observed, leading to formation of particles of some hundreds of $\mathrm{nm}$ 
in the solution. Thus, one of the possible reasons for poor enhancement of AuNPs on fibrils could be rapid nucleation and reduction of the gold in solution, which leads to preferential enhancement of the gold particles in the solution and not on the gold particles attached to the fibrils.
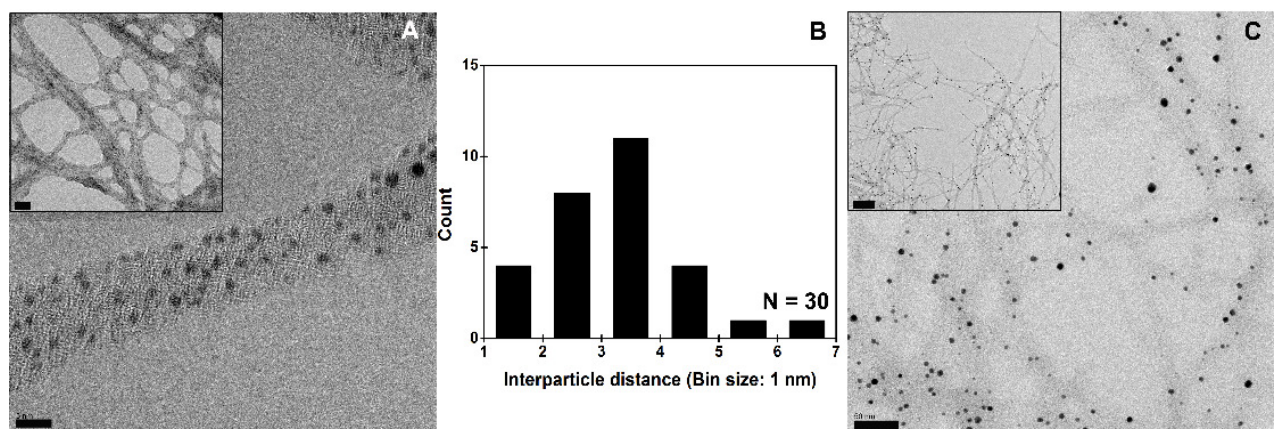

Figure 6.5 (A) TEM image of A140C fibrils assembled from unlabeled monomers and then labeled with $1.4 \mathrm{~nm}$ AuNPs, scale bar $5 \mathrm{~nm}$. Inset: overview image, scale bar: $50 \mathrm{~nm}$. (B) Histogram showing interparticle distance on fibrils in " $A$ ". (C) Electroless gold enhancement on fibrils in " $A$ ", showing non-uniform enhancement in the size of AuNPs attached to the surface of the fibrils, scale bar: $50 \mathrm{~nm}$. Inset: overview image, scale bar: $200 \mathrm{~nm}$.

\section{Labeling with $5 \mathrm{~nm}$ AuNPs in solution}

Next we explored labeling the fibrils with $5 \mathrm{~nm}$ colloidal AuNPs with the view that bigger particles may be better nuclei for electroless gold enhancement in comparison to $1.4 \mathrm{~nm}$ AuNPs. Owing to the affinity of the -SH group to the gold surface, the thiol group of the terminal cysteine in the $\mathrm{A} 140 \mathrm{C}$ monomer is expected to form a covalent bond with gold. The surface of the $5 \mathrm{~nm}$ colloidal gold particles used was citrate stabilized imparting a negative surface charge to the gold particles (Bellino, Calvo et al. 2004; Levy, Thanh et al. 2004; Sperling and Parak 2010). Therefore, exploiting the affinity of thiol towards gold and the negative surface charge, we investigated two methods for attachment of $5 \mathrm{~nm}$ colloidal AuNPs to A140C aSyn fibrils. Firstly, we coincubated an aggregation reaction of A140C aSyn monomers with $5 \mathrm{~nm}$ colloidal AuNPs (labeling during fibrillization) and secondly, we tried labeling the fibrils using electrostatic interactions and thiol-gold affinity (labeling assembled fibrils). 


\section{Labeling during fibrillization}

A140C monomers were fibrillized in the presence of $5 \mathrm{~nm}$ AuNPs but the ThT assay, akin to the results of the $1.4 \mathrm{~nm}$ AuNPs aggregation with 1:1 labeling ratio, did not show any increase in the ThT fluorescence (data not shown). However, AFM images of these samples show short amyloid like fibril fragments, and exhibit lateral fibril clustering (Figure 6.6A (control) and B). The absence of ThT fluorescence in the fibril containing sample could be due to low level of $\beta$-sheet structure (i.e. fibrils) or because of a partial overlap in the emission spectrum of ThT (emission maximum at $485 \mathrm{~nm}$ ) and the absorption spectrum of the AuNPs (absorption maximum at 510-525 nm), resulting in energy transfer and a decrease in ThT fluorescence. Formation of long fibrils could have been impeded by thiol-gold interaction between the cysteine carrying monomers and the gold particles. Interaction between the soluble monomers and the gold particles can be imagined to affect fibrillization. $5 \mathrm{~nm}$ gold particles may cap the ends of the fibrils resulting in short fragments, as observed in the AFM images (Figure 6.6B).
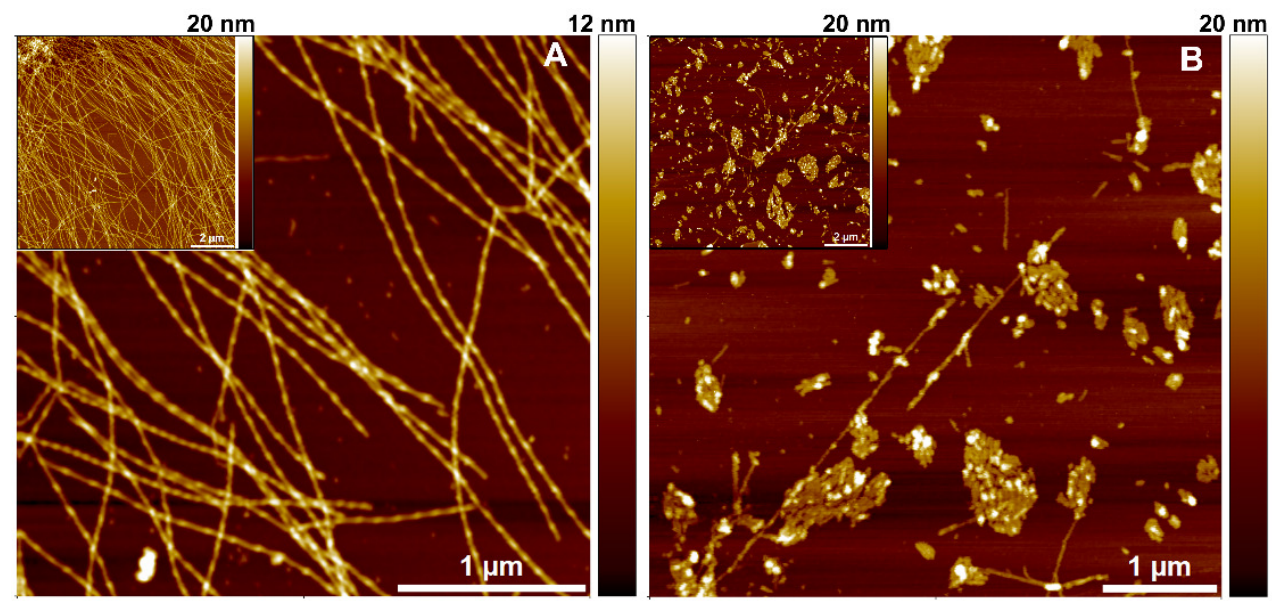

Figure 6.6 (A) Control AFM height image showing fibrils assembled in the absence of gold particles. Inset: overview image. (B) Fibrils prepared in the presence of $5 \mathrm{~nm}$ colloidal gold particles. Inset: overview image. 


\section{Labeling of assembled fibrils}

Assembled fibrils were labeled with $5 \mathrm{~nm}$ AuNPs based on electrostatic attraction and the thiol-gold affinity by choosing appropriate reaction conditions. The electrostatic attraction was used as a bait to bring the thiol and the gold particles closer to facilitate formation of a thiol-gold covalent bond. A140C monomers were assembled into fibrils as mentioned above. Fibrillization of A140C aSyn at $\mathrm{pH} 7.4$ results in negatively charged fibrils, because the constituting protein molecules have a pI of about 4.7. The 5 $\mathrm{nm}$ AuNPs used were stabilized by citrate and thus imparted a negative charge to the surface of the gold particles (Bellino, Calvo et al. 2004). To increase the electrostatic attraction between the negatively charged fibrils and the $5 \mathrm{~nm}$ AuNPs, the charge of the fibrils was reversed by changing the buffer to $10 \mathrm{mM}$ sodium acetate solution at $\mathrm{pH}$ 3.6. Next, the fibrils were mixed with $5 \mathrm{~nm}$ AuNPs with gentle stirring.

TEM imaging on the samples showed that the $5 \mathrm{~nm}$ AuNPs attached preferentially along the edges of the fibrils (Figure 6.7A). Moreover, not all the fibrils were labeled with the colloidal gold particles. A moderate proportion of the fibrils appear to be unlabeled in the background in TEM images. The multiple steps of centrifugation and resuspension in the protocol were observed to induce clustering of fibrils. Based on this observation, it can be imagined that the inner fibrillar mass in such clusters may not be
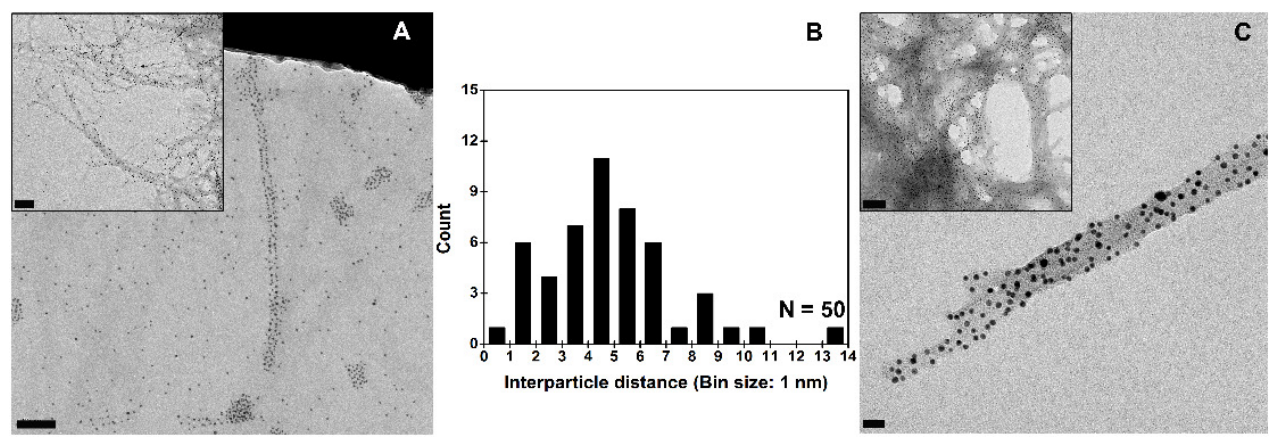

Figure 6.7 (A) TEM image showing fibrils labeled with $5 \mathrm{~nm}$ AuNPs. The gold particles attach along the edges of the fibrils and most of the fibrils in the background, visible as light gray filaments, do not appear to be labeled, scale bar: $100 \mathrm{~nm}$. Inset: overview image, scale bar: $200 \mathrm{~nm}$. (B) Histogram showing distribution of the interparticle distance of $5 \mathrm{~nm}$ AuNPs on fibrils in "A". (C) TEM image of fibrils sequentially labeled with 5 and $1.4 \mathrm{~nm}$ AuNPs, scale bar: $20 \mathrm{~nm}$. Inset: Overview image, scale bar: $100 \mathrm{~nm}$. 
equally accessible to the solution in comparison to the fibrils on the outer surface of the cluster. Therefore, reduced solvent accessibility can be speculated to be a likely cause for the poor labeling of the fibrils.

The average center to center interparticle distance between the gold particles on the labeled fibrils was calculated to be $4.8 \pm 2.4 \mathrm{~nm}$ (Figure 6.7B). Thus, the density of the gold particles is lower when labeled with $5 \mathrm{~nm}$ colloidal particles in comparison to when labeled with $1.4 \mathrm{~nm}$ AuNPs $(3.3 \pm 1.7 \mathrm{~nm})$. The lower density of gold particles is even evident in their arrangement along the edges of the fibrils, which shows only 2 gold particles across the transverse section of the fibrils. The low density can be conjectured to be due to probable steric hindrance because of the size of the particles i.e. $5 \mathrm{~nm}$ with respect to the diameter of $\alpha$ Syn fibrils, which is typically $7 \mathrm{~nm}$. However, this also indicates that the fibrils labeled with $5 \mathrm{~nm}$ AuNPs should have free cysteines on the fibril surface. If this supposition is true then $1.4 \mathrm{~nm}$ AuNPs should be able to conjugate on the fibrils labeled with $5 \mathrm{~nm}$ AuNPs and produce fibrils decorated with two sizes of gold particles.

Next, we did a two-step gold labeling on the fibrils. First we labeled the fibrils with $5 \mathrm{~nm}$ colloidal gold particles and then with $1.4 \mathrm{~nm}$ AuNPs. TEM images reveal that, as expected, $1.4 \mathrm{~nm}$ AuNPs labeled the fibrils and produced hybrid fibrils with AuNPs of two sizes, 5 and $1.4 \mathrm{~nm}$ AuNPs (Figure 6.7C)

The fibrils hybridized with 1.4 and $5 \mathrm{~nm}$ AuNPs appeared to have a high density of gold particles on the surface, and therefore probably have conductive properties. To assess this possibility, bulk conductivity measurements using micro-interdigitated electrodes (microIDTs) were tried on the labeled fibril samples. Fibril samples were deposited by drop casting on the silicon based microIDTs. Unfortunately the samples did not adsorb well on the silicon surface and the conductivity measurements were not successful.

\section{Functionalization of $\alpha$ Syn fibrils with AuNPs on a substrate}

To circumvent the problems associated with clustering of the fibrils (which result in poor electroless gold enhancement and labeling of $5 \mathrm{~nm}$ AuNPs) and weak adsorption on the silicon based interdigitated electrodes, we performed functionalization of the fibrils adsorbed on compatible substrates: mica and TEM grids. Although the substrate adsorbed fibrils present a 2 dimensional surface for functionalization, the immobilized 

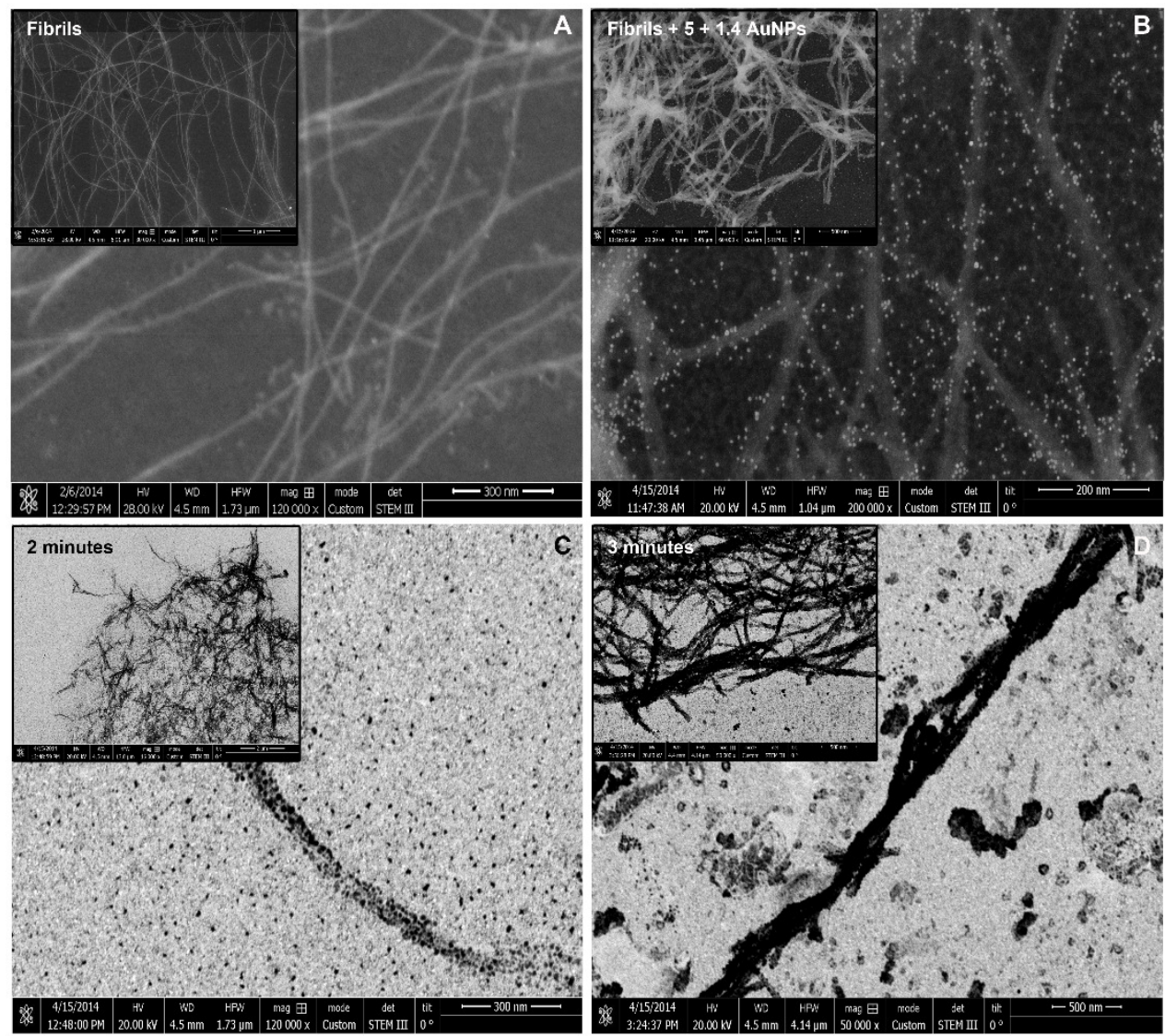

Figure 6.8 STEM images of fibrils functionalized on a TEM grid. (A) Fibrils with no gold particles (B) fibrils labeled with 5 and $1.4 \mathrm{~nm}$ AuNPs (C and D) Fibrils in "B" exposed to electroless gold enhancement at $60^{\circ} \mathrm{C}$ for (C) 2 minutes and (D) 3 minutes.

fibrils cannot cluster or break since centrifugation and resuspension steps are not required to change the reaction buffers.

Optimization for the gold functionalization and enhancement was done on TEM grids, as it is easier and more accurate to assess the enhancement using electron microscopy than with AFM. Fibrils were adsorbed on a TEM grid and functionalized with 1.4 and $5 \mathrm{~nm}$ AuNPs. The electroless gold enhancement was performed at $60{ }^{\circ} \mathrm{C}$ instead of room temperature. At higher temperature, due to increased thermal motion, the probability of interaction between the reducing gold ions and the gold particles conjugated on the fibril surface is higher. Thus, the process of enhancement can be 
expected to be more efficient. Functionalization of the fibrils on the substrate resulted in dispersed fibrils labeled with AuNPs. The enhancement of the gold particles was decidedly more efficient than when performed in solution (Figure 6.8). Electroless gold enhancement for 2 minutes resulted in an increase in the size of the AuNPs (Figure 6.8C). Further enhancement to a total of 3 minutes resulted in a dense arrangement of gold on the fibrils; such that the individual particles were no longer distinguishable (Figure 6.8D). Therefore, functionalization and electroless gold enhancement of the fibrils with AuNPs is superior when the fibrils are adsorbed on a suitable substrate, like a TEM grid, than in solution.

\section{Conductivity measurements}

Next, we examined the conductive properties of the gold functionalized fibrils on a mica substrate using conductive probe AFM. The fibrils were deposited on freshly cleaved mica and functionalized as described in the previous section with 3 minutes of electroless gold enhancement. A part of the mica surface was shadow masked to create a gold-mica interface. The concept of the experiment was that the AuNPsfunctionalized fibrils adsorbed at the gold-mica interface, when scanned with a conductive AFM tip, with an applied bias voltage, should show a current signal in the current channel of the conductive probe AFM. By scanning along the length of the fibrils, changes in the conductive properties with different bias voltages and forces could be studied.

AuNPs labeled fibrils adsorbed on the mica surface (with gold sputtered masked and unmasked sections) were tested for their conductive properties by conductive probe AFM. First, the AuNPs labeled fibrils were probed in contact mode. However, imaging in contact mode was unsuccessful as after scanning a few lines of the image the signal in the current channel was lost, while the height channel still showed a typical height image. This interruption of the signal in the current channel could be due to damage to the conductive layer of the tip or the contamination of the tip with non-conductive particles. One of the probable reasons for the tip damage could be the friction it experiences in contact mode. To avoid friction related complications, the AuNPs labeled fibrils were then imaged in peak force tapping mode using conductive probe AFM. 
Using an optical microscope coupled to the AFM, the AFM tip was positioned over the edge of the sputtered gold and mica. The tip was engaged to the surface and the gold-mica edge was located. Imaging in tapping mode produced stable images in the height as well as in the current channel. Although during sputtering about $30 \mathrm{~nm}$ of gold was deposited on the mica to create a razor-edge, at the nanoscale the edge appeared to be very diffuse. In control samples, with sputtered gold and unlabeled fibrils, no step could be observed at the gold-mica interface in the height channel (Figure 6.9A and inset). In the current channel, however, a conductive and nonconductive partition corresponding to the gold and mica surface could be observed (Figure $6.9 \mathrm{~B}$ and inset). At a DC sample bias voltage of $100 \mathrm{mV}$ and a tapping force of

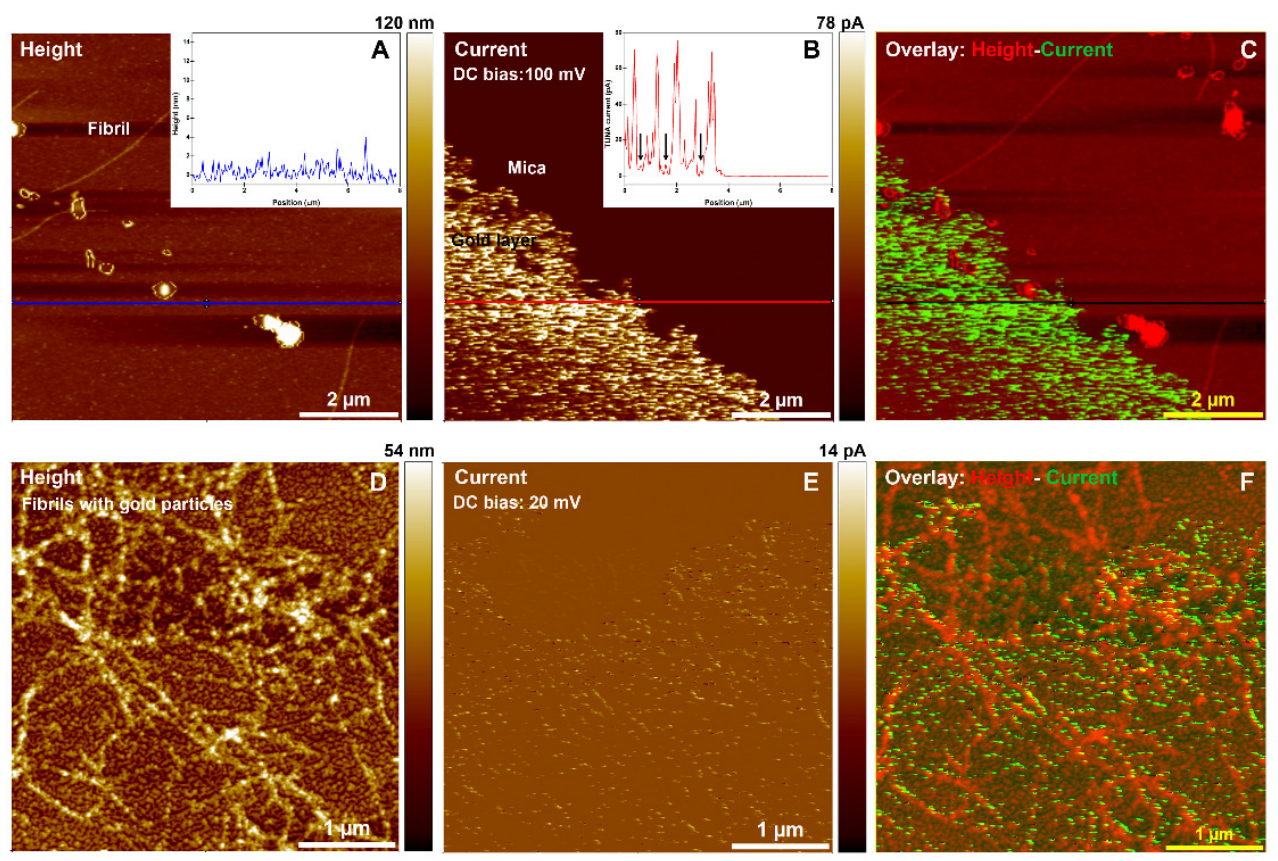

Figure 6.9 Representative conductive probe AFM images. Upper panel: control sample with unlabeled fibrils at the gold-mica interface. (A) Height image. Inset: height profile across the blue line shown in the height image. (B) Current image displaying conductive (gold) and insulating (mica) parts of the sample. Inset: current profile across the red line shown in the current image. Black arrows indicate the insulating pockets in the conductive layer. (C) An overlay of height and the current channel. Lower panel: sample with gold labeled fibrils (D) Height image showing network of gold labeled fibrils (E) Current image exhibiting conductive signal (F) An overlay of height and the current channel demonstrating correlation of the fibrils and the conductive signal. 
$30 \mathrm{nN}$, the conductive layer showed a maximum current of about $70 \mathrm{pA}$ (Figure 6.9B: inset). The current measured in the sputtered layer is about 9 orders of magnitude lower than that expected for metallic conduction (resistance $\approx 1.4 \mathrm{G} \Omega$ ). The observed low current thus does not follow ohmic behavior.

In samples with gold labeled fibrils, the height channel showed the presence of fibril networks and the current channel showed a current signal in pico-amperes (Figure 6.9D and E). Correlation of the height and the current channel images showed overlap of the two signals, suggesting current detection through the fibrils (Figure 6.9F). However, not all the fibrils observed in the height channel showed a corresponding current signal. At the applied DC bias voltage of $20 \mathrm{mV}$ and a tapping force of $20 \mathrm{nN}$, tens of pico-amperes of current was detected (Figure 6.9E). As in the control samples, the current measured through the functionalized fibrils was about 9 orders of magnitude lower than that expected for metallic conduction with a high resistance of $\approx 1.4 \mathrm{G} \Omega$. Similar quantitative values in the control and the gold labeled fibril sample suggests that the flow of electrons in the present experimental design is most likely limited due to the sputtered gold layer, designed to act as one of the electrodes.

It appears that at the gold-mica interface, the sputtered gold layer has no sharp edge but extends with a very shallow gradient over a large area. The gold layer is unlikely to be more than a few atomic layers thick, as it cannot be detected in the height channel of the AFM image, which can reliably detect height variations close to $0.5-1 \mathrm{~nm}$. Moreover, a cross-sectional profile of the conductive (gold) layer in the control sample shows that the conductive surface is not continuous but has insulating pockets (Figure 6.9B: inset indicated by black arrows). Thus being thin and porous, the sputtered gold layer presents a random conductive surface, which can stochastically transmit electrons. As a result, the conductive properties of the gold labeled fibrils may be superior to the sputtered gold layer, but they cannot be studied accurately with the current experimental design. Nonetheless, the presented experiments show that the aSyn fibrils functionalized with gold nanoparticles are conductive and demonstrate the sensitivity of the conductive probe AFM to extremely low currents. To improve the experiment design, a sharp gold-mica interface with at least a few nm thick and continuous gold surface must be created. 


\section{CONCLUSIONS}

In this chapter, we show that fibrils with exposed cysteine residues on their surface can be functionalized with 1.4 and $5 \mathrm{~nm}$ AuNPs or particles of both sizes resulting in a hybrid bionanostructure. To make these hybrid structures conductive, the density of the gold on the fibril surface can be further increased by electroless gold enhancement. Preliminary investigation of the thus produced gold labeled fibrils with a conductive probe AFM strongly suggests the presence of a conductive gold layer on the functionalized fibrils.

\section{ACKNOWLEDGEMENTS}

We are grateful to the following people for their invaluable assistance: Kirsten van Leijenhorst-Groener, Yvonne Kraan and Nathalie Schilderink for protein expression and purification. Dr. Ron Gill for learned information about gold nanoparticles.

Dr. Martin Bennink, Kees van der Werf and Robert Molenaar for insightful discussions on AFM imaging and interpretation of the images. Robert Molenaar for gold sputtering of the samples for conductivity experiments.

Dr. Rico Keim and Mark Smithers for the TEM imaging at the MESA+ NanoLab facility at the University of Twente.

STEM images were acquired at the nanoCenter AMOLF. We thank Andries Lof for training Arshdeep on the SEM Verios instrument and helping with imaging.

Conductivity experiments were done in collaboration with the Nano-Electronics group at the University of Twente. We are grateful to Prof. Wilfred van der Wiel for the discussions. Serkan Büyükköse performed the conductivity experiments on the microinterdigitated electrodes. Martin H. Siekman and Robert R. Wijn performed the conductive probe AFM experiments at the MESA+ NanoLab facility at the University of Twente, and helped with the interpretation of the results. 


\section{Chapter 7}

\section{Discussion and future perspectives}

In this chapter we discuss the key results of the research described in this thesis and deliberate on possible interesting follow up experiments. 


\section{POLYMORPHISM AND AMYLOID STUDIES}

Self-assembly of soluble monomeric proteins into amyloid form has been a topic of intense investigation for the past decades. On one hand, the association of amyloids with a number of degenerative diseases like Alzheimer's, Parkinson's, Huntington's disease, type II diabetes etc., positions them as a key therapeutic target for prevention, treatment and disease management (Knowles, Vendruscolo et al. 2014). On the other hand the property of spontaneous self-assembly, in optimal solution conditions, coupled with desirable material properties makes amyloids valuable for development of bio-nanostructures (Mankar, Anoop et al. 2011). However, in practice, both aspects of amyloid science - that is, finding a cure for pathogenic amyloids and developing amyloids as novel nanomaterials - are considerably limited by the lacunae in our understanding of the molecular mechanisms/factors that trigger and sustain the amyloid form in proteins. One of the challenges in studying amyloid proteins has been the inherent structural polymorphism of amyloid fibrils (Goldsbury, Frey et al. 2005; Heise, Hoyer et al. 2005; Fandrich, Meinhardt et al. 2009; Tycko 2014). The presence of multiple amyloid forms in a sample is a major setback for structural and toxicity studies, as distinct polymorphs have been suggested to exhibit different biochemical and biophysical properties (Yoon, Lee et al. 2014; Peelaerts, Bousset et al. 2015).

Optimizing solution conditions to suppress heterogeneity: In chapter 2 we studied the fibrillization of the aSyn protein with the goal to reduce the morphological heterogeneity via selective solution conditions. We found that very low salt conditions of $1 \mathrm{mM} \mathrm{NaCl}$ produced more homogeneous fibrils but are not optimal due to very slow aggregation kinetics. A ten times higher, but relative to physiological salt conditions still low, $\mathrm{NaCl}$ concentration of $10 \mathrm{mM}$ produced fibrils with heterogeneous periodicities but inclusion of $0.1 \mathrm{mM}$ EDTA reduced the heterogeneity markedly. The homogeneous fibrils produced were studied for polymorph specific features in the following chapters. Polymorphism is believed to arise due to conformational flexibility of the soluble aSyn monomers. aSyn, being an intrinsically disordered protein, is suggested to have a higher degree of conformational freedom in comparison to folded proteins (Dunker, Brown et al. 2002). Despite being categorized as an intrinsically disordered protein, $\alpha$ Syn has been proposed to exist in an ensemble of collapsed coil structures with long range intramolecular interactions (Dedmon, Lindorff-Larsen et al. 2005). These interactions 
are believed to be governed by hydrogen bonds and electrostatic interactions. Therefore inclusion or removal of salt and metal ions can be imagined to affect these interactions. In our experiments in chapter 2 we do not add any metal ion and thus we believe that the homogenization of the morphology observed in the presence of EDTA is due to chelation of trace metal ions, which results in the reconfiguration of aggregation prone conformations. It would thus be interesting to examine the effect of specific metal ions like $\mathrm{Fe}^{2+/ 3+}, \mathrm{Cu}^{2+}, \mathrm{Zn}^{2+}$, which are reported to be present in relatively high concentrations in PD patient brain samples, on fibril polymorphism. Furthermore, if inclusion of specific metal ions, at a given concentration, can lead to emergence of new polymorph(s), it is likely to provide further insights into mechanisms regulating polymorphism.

Fibrillization mechanisms: Since the discovery of aSyn fibrils as the major component of Lewy bodies, numerous studies have shown that recombinantly produced aSyn monomers readily self-assembly into fibrils in vitro (Spillantini, Schmidt et al. 1997; Spillantini, Crowther et al. 1998; Giasson, Uryu et al. 1999; Wood, Wypych et al. 1999; Conway, Harper et al. 2000; Hoyer, Antony et al. 2002). The fibrils formed were initially proposed to be the same as those found in PD patients (Giasson, Uryu et al. 1999; Conway, Harper et al. 2000). The fibrils appeared as unbranched elongated filamentous structures. However, successive studies with improved resolution of EM, ssNMR and AFM have shown that the in vitro produced fibrils are not identical in their morphology and exhibit intrinsic polymorphism (Petkova, Leapman et al. 2005; Paravastu, Qahwash et al. 2009). The polymorphs of A $\beta$ and $\alpha$ Syn were further shown to manifest characteristic structural signatures in terms of conformations of the constituent protein monomers (Heise, Hoyer et al. 2005; Petkova, Leapman et al. 2005; Paravastu, Leapman et al. 2008; Vilar, Chou et al. 2008; Gath, Bousset et al. 2014). Interestingly, recent studies on $A \beta$ suggest that fibrils examined from patient derived samples are not heterogeneous and have a principal morphology, which may vary between patients (Lu, Qiang et al. 2013; Tycko 2014). To resolve the observed dichotomy between the patient-derived samples (homogeneous) and in vitro aggregations (heterogeneous) it is important to understand the basic mechanisms that govern the process of fibrillization.

In chapter 3, we showed that irrespective of the protein sequence, aSyn fibril formation proceeds via a competitive growth mechanism, which results in a 
homogeneous fibril population at the plateau phase. Moreover, the wt aSyn fibrils, in contrast to stable mutant fibrils, appeared to mature over time. It is likely that the observed competitive growth and maturation process occurs during disease progression as well. The stable nature of the fibrils from aSyn disease mutants could present surfaces for surface catalyzed aggregation and result in increased aggregation of the monomers (Meisl, Yang et al. 2014). Such a behavior may contribute to the accelerated and extensive aggregation observed in familial forms of PD (Conway, Harper et al. 1998; Conway, Harper et al. 2000). However, to methodically extend the results of chapter 3 , the competitive growth and the maturation of the fibrils, to a physiologically relevant condition, wt and the disease mutant aSyn aggregations should be studied in physiologically relevant milieu apropos the protein and salt concentration, and at physiological temperature.

The maturation process observed in the wt fibrils presents a complex interplay of kinetically and thermodynamically favored conformations of the protein monomers. Selection of one of the polymorphs during the aggregation process and the ensuing maturation over 3-6 months represents a conflict between the kinetically and the thermodynamically favored conformations. The precise mechanism of the transition between these two conformations is difficult to elucidate without high resolution structural studies. We propose that high resolution structural studies like ssNMR, cryoEM and X-ray crystallography should be performed on the plateau phase and the matured fibrils. In addition to understanding the maturation process, structural information can be instrumental in distinguishing subtle yet critical conformational differences that yield fascinatingly diverse biochemical and biophysical characteristics to the polymorphs.

Polymorph specific characters: One of the biochemical differences described in chapter 4 is the sensitivity of the ThT fluorescence intensity to the aSyn fibril morphology. Using seeded aggregations over two generations that resulted in aSyn fibrils with identical chemical composition but distinct morphology we could successfully demonstrate that the observed ThT fluorescence intensity depends on the fibril morphology. Factors that determine the ThT fluorescence on binding to fibrils are not well understood. It has been proposed that the fibril surface presents more than one binding site for the ThT molecules (LeVine 1999). In our results using aSyn fibril populations with different morphologies, the fluorescent lifetimes were similar at room 
temperature but the relative distributions of the two distinct binding sites were different. Based on literature reports (LeVine 1999; Krebs, Bromley et al. 2005; Lockhart, Ye et al. 2005; Wu, Biancalana et al. 2009; Biancalana and Koide 2010), the quantitative and qualitative features of the binding sites seem to be determined by the protein sequence. In our results, in addition to temperature which clearly influenced ThT lifetimes and the relative amplitudes in both the aSyn fibril samples at $80^{\circ} \mathrm{C}$, the concentration of ThT and the surface of the fibrils also appeared to play a role in fibrilThT interaction. In literature, the chirality of the fibrils has been suggested to affect the binding of ThT molecules to the fibrils, resulting in an ICD signal at $450 \mathrm{~nm}$ (Dzwolak and Pecul 2005; Loksztejn and Dzwolak 2008). In our experiments, although the A30P and A53T fibrils showed different morphologies with possibly distinct handedness in the AFM images, we could not obtain consistent results with ICD measurements. Thus, the rationale that the chiral surface of the fibril provides a chiral binding site to the incoming ThT molecule could not be confirmed. Furthermore, the concentration of the fibrils also appeared to affect the orientation of the ICD signal, thereby suggesting fibrilThT interactions to be dynamic. The dynamic relation of the fibril-ThT interactions is also demonstrated by the drop in the lifetimes of ThT with increasing ThT concentration in the sample (dynamic quenching). However, the change in the relative amplitudes of the two lifetime components is intriguing. Follow up studies investigating the relation between fibril concentration and ThT fluorescence lifetimes could provide better understanding of how the changes in the aSyn fibril mass with the progression of aggregation affects the read out of the ThT fluorescence assay.

Fidelity in heterologous aggregations: Another interesting aspect of the polymorphic fibrils in an aggregation is how the presence of one polymorph affects the other. The results of chapter 3 suggested that there is competition between the polymorphs for the monomer pool during fibrillization. One of the complicating factors in the cellular milieu could be the occurrence of different proteins with similarity in sequence and/or conformation(s). Since amyloids are increasingly linked with prionlike properties, the question if a polymorph can recruit monomers of different proteins sequences is exciting. The results from chapter 5 showed that successful heterologous seeding between aSyn sequences is driven by the compatibility between the seed and the soluble monomer conformations. In seeded aggregations, the morphology of the resultant fibril and the growth characteristics (unidirectional or bidirectional) are 
guided by the seed properties. However, considering the deformed templating proposed for prions (Makarava and Baskakov 2013), in aggregations between seeds and monomers with not only different conformation (conformational preference for monomers) but also different sequences, the fidelity of templating may not be stringent over generations of aggregations. Deformed templating has been suggested as one of the mechanisms for the generation of new strains (with typical phenotype and genotype) and their propagation. It would be interesting to observe if deformed templating can be observed in a cascade of aSyn seeded aggregations in cell-based studies.

One of the possible experiments could test if Lewy body formation can be induced in neuronal cell lines by different polymorphs, which can be specifically detected using conformation sensitive probes (like luminescent conjugated oligothiophene probes (Nystrom, Psonka-Antonczyk et al. 2013)). Then a comparative study over a number of passages in the presence of monomers (with similar and different conformational preferences) may provide clues if heterologous aggregation occurs in cells and how strictly the conformation of the seed is preserved over time.

\section{CONDUCTIVE HYBRID NANOSTRUCTURES}

Given the attractive material properties of amyloids, with the additional advantage of spontaneous self-assembly and nanoscale dimensions, great efforts have been made to functionalize them. In most of the functionalization studies the amyloids mostly act as a scaffold, while components like metal nanoparticles, fluorophores, electrolytes etc. provide the desired functionality to the hybrid structure (Scheibel, Parthasarathy et al. 2003; Colby, Hulleman et al. 2008; Bjork, Herland et al. 2010; Knowles, Oppenheim et al. 2010; Lee, Choe et al. 2011; Muller, Jansson et al. 2011). In addition to the generic properties of amyloids that arise due to a common mechanism of fibrillization i.e. the formation of cross $\beta$-sheet structure, the polypeptide specific features are also important. In an earlier report on functionalization of a peptide of Sup35p the authors found solution conditions for aggregation where fibrils arranged in parallel arrays (Scheibel, Parthasarathy et al. 2003). The resulting hybrid structures were thus single fibrils functionalized with gold particles. In our experiments in chapter 6 we first strived to functionalize aSyn fibrils with 1.4 and $5 \mathrm{~nm}$ AuNPs followed by electroless gold enhancement of the fibrils suspended in solution to achieve conduction competent 
assemblies. However, the functionalized fibrils showed a high propensity to cluster. Therefore the functionalized $\alpha$ Syn fibrils were present as a network rather than as single fibrils. Furthermore the clustering was believed to lead to poor electroless enhancement, thus, making the functionalization unpredictable. Identifying solution conditions that minimize self-association of $\alpha$ Syn fibrils would be essential to producing single fibrils functionalized with gold particles in a three dimensional configuration.

To circumvent the issues related to clustering and inefficient labeling we performed functionalization of fibrils with 1.4 and $5 \mathrm{~nm}$ AuNPs deposited on a substrate. Two different substrates were tried: carbon-coated TEM grids to optimize the functionalization and electroless gold enhancement, and mica. Fibrils showed less clustering and remarkably improved the electroless gold enhancement upon adsorption to a substrate. For probing the conductive properties of the functionalized fibrils we used gold microIDTs on a silicon wafer. While the fibrils adsorbed well on the TEM grid and mica, adsorption on microIDTs was unsuccessful. Thus measurement of the conductive properties of the functionalized aSyn fibrils posed a challenge. Next, contact and tapping modes of conductive probe AFM were tested to measure the conductive properties of the fibrils. Tapping mode produced more stable images in comparison to contact mode and was chosen for subsequent experiments. The image in the current channel representing the conductive properties of the sample showed conductive fibrils but not all fibrils showed transmission of electrons. The magnitude of the current measured was very low; in pico-amperes with a bias voltage of milli-volts. Examination of the sputtered gold layer, acting as one of the electrodes, revealed that the gold layer was very thin and discontinuous. Similar conductive features of the gold layer in the control sample strongly suggested that the experimental design was limiting detection of superior conductive properties of the functionalized fibrils, if any. As a follow up to the experiments, we can suggest two different strategies to probe the conductive behavior of the fibrils.

Firstly, microIDTs could be fabricated on mica instead of silicon. The fibril samples show good adsorption on the mica surface thus the problem of adsorption can be tentatively overcome using mica as the substrate (Figure 7.1). However, since mica is a rather fragile material due to the ease of cleavage, caution must be exerted not to damage the fabricated layer. 


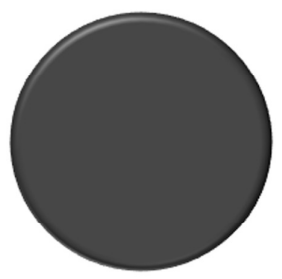

Mica surface

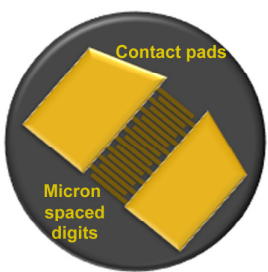

Fabrication of microlDTs on mica

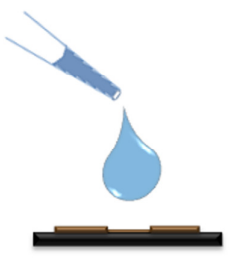

Sample deposition

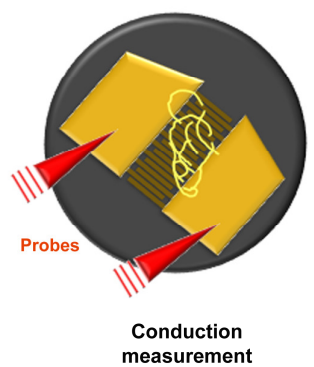

Figure 7.1 Schematic representation of the experiment design to probe conductive properties of functionalized aSyn fibrils using microIDTs fabricated on a mica substrate.

Secondly, multi-probe AFM could be used to measure the current between two points on a fibril (Figure 7.2). Use of multi-probe AFM does not require a sputtered electrode making the experiment in principle simpler and more direct.

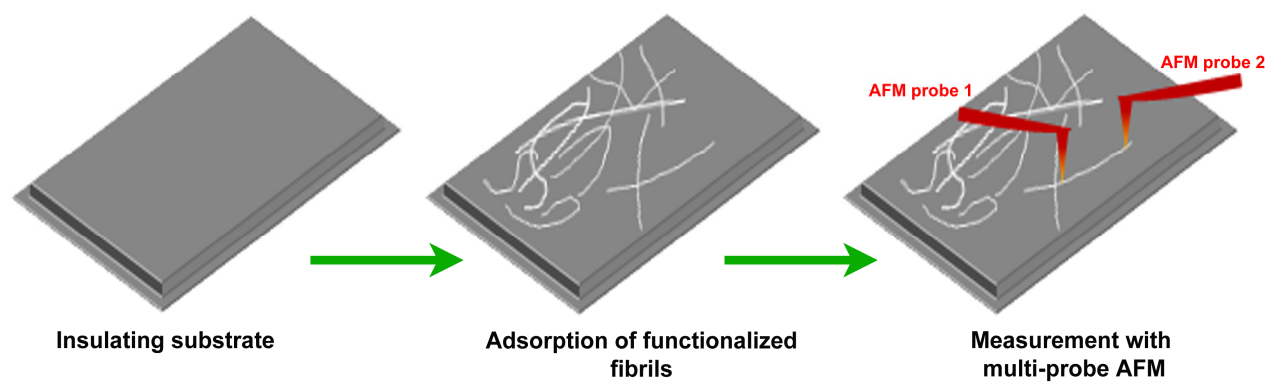

Figure 7.2 Schematic representation of the experiment design to probe conductive properties of functionalized $\alpha$ Syn fibrils using multi-probe AFM.

Although our experiments to prepare metallic conductive fibrils (nanowires) were not an outright success, we learnt a great deal about handling the fibrils for functionalization and the technical challenges for measuring their conductivity. We believe that by building on the insight from the different experiments, fibrils with better conductive properties can be prepared and efficiently measured. However, given the rising concern over the prion-like properties of $\alpha$ Syn other amyloid systems (synthetic peptides) not associated with disease might be a safer choice for scaffold. 


\section{Appendix A}

\section{Risk analysis of gold nanoparticles}

The safety of the gold nanoparticles used in the functionalization of aSyn fibrils in this thesis is not established. In this appendix we analyze the risk associated with gold particles used in chapter 6 . 
The process of risk analysis includes risk assessment, risk management and risk communication (Miller and Wickson 2015). Risk assessment of nanoparticles can be described as an evaluation of the probability of developing adverse health or environmental effects due to exposure to a certain dose for a given period. Once the risk has been estimated, strategies (risk management) can be drawn to minimize it. A crucial aspect of risk management is creating awareness among those at risk (risk communication). The exposure and the risk posed by AuNPs can be evaluated at two levels:

Direct exposure: Individuals can be exposed to gold nanoparticles (AuNPs) directly in two situations. Occupational/personnel exposure, people working in industries manufacturing AuNPs are at high risk of exposure due to long and repeated work hours in the vicinity of high concentrations of AuNPs. Secondly, as consumers/personal exposure, when using the AuNPs in research and development (R\&D) or in products containing AuNPs like cosmetics, food additives, paints and surface coatings (Miller and Wickson 2015).

Indirect exposure via the environment: Environmental components like air, water and soil are the eventual sink for AuNPs at different stages of their life cycles. The processes involved in synthesis, $\mathrm{R} \& \mathrm{D}$, and usage of products containing nanoparticles (NPs) introduce NPs into the environment as active waste as well as passive waste (for example, by leaching from the product while in use).

\section{ASSESSMENT OF EXPOSURE TO AUNPS USED IN CHAPTER 6}

Quantitative risk assessment of AuNPs is not well formulated thus it is very difficult to evaluate the exact risk involved in using AuNPs in the laboratory. Moreover, absence of guidelines for handling and waste disposal leaves it to the individual researcher to estimate the risk and decide on the safety measures that should be followed. Thus structured information on the potential risks and comprehensive regulations for usage and waste disposal of AuNPs would be highly valued.

Based on our experience in the laboratory and the information we could find in literature, here we try to assess the potential risks of the AuNPs used for functionalization of aSyn fibrils in chapter 6. Two types of commercially available AuNPs were used for the functionalization studies: 1.4 and $5 \mathrm{~nm}$ diameter particles. 
Some cell and animal studies suggest potential toxic effects of AuNPs of these dimensions (summarized in Table A.1).

Table A.1 Summary of studies on nanotoxicity evaluation of 1.4 and $5 \mathrm{~nm}$ gold nanoparticles.

\begin{tabular}{|c|c|c|c|c|c|}
\hline $\begin{array}{l}\text { AuNP } \\
\text { Size }\end{array}$ & Toxicity & Dose & $\begin{array}{l}\text { Exposure } \\
\text { duration }\end{array}$ & Model system & Ref \\
\hline $\begin{array}{c}\mathrm{Au}_{55} \\
1.4 \mathrm{~nm}\end{array}$ & Toxic & $0.4 \mu \mathrm{M}$ & $24 \mathrm{~h}$ & $\begin{array}{l}\text { Various cell } \\
\text { lines }\end{array}$ & $\begin{array}{l}\text { (Liu, Meyer-Zaika et al. } \\
\text { 2003; Tsoli, Kuhn et al. } \\
\text { 2005) }\end{array}$ \\
\hline $1.4 \mathrm{~nm}$ & Toxic & Varied & $48 \mathrm{~h}$ & HeLa cells & (Pan, Leifert et al. 2009) \\
\hline $1.5 \mathrm{~nm}$ & $\begin{array}{l}\text { TMAT: Toxic } \\
\text { MES: Teratogenic } \\
\text { MEEE: No effect }\end{array}$ & $\begin{array}{c}250 \\
\mu \mathrm{g} / \mathrm{ml}\end{array}$ & $120 \mathrm{hpf}$ & $\begin{array}{l}\text { Zebra fish } \\
\text { embryos }\end{array}$ & $\begin{array}{l}\text { (Truong, Tilton et al. } \\
\text { 2013) }\end{array}$ \\
\hline $5 \mathrm{~nm}$ & No & $8 \mathrm{mg} / \mathrm{kg}$ & 21 days & BALB/c Mice & (Chen, Hung et al. 2009) \\
\hline $5 \mathrm{~nm}$ & No & $\begin{array}{c}10^{-4} \text { to } 0.1 \\
\mathrm{mM}\end{array}$ & NA & HeLa cells & (Chen, Hung et al. 2009) \\
\hline $5 \mathrm{~nm}$ & toxic & $50 \mu \mathrm{g} / \mathrm{ml}$ & $48 \mathrm{~h}$ & A549 and 95D & (Liu, Wu et al. 2014) \\
\hline
\end{tabular}

Therefore, it is important that precautions be observed to avoid exposure to the AuNPs as much as possible. Some of the potential sources of exposure and the estimated risks are discussed as follows.

\section{Synthesis}

The two types of AuNPs used in the present study, $1.4 \mathrm{~nm}$ monomaleimide nanogold particles (Cat \# 2020A Monomaleimido Nanogold (MMNA) from Nanoprobes) and 5 nm colloidal gold particles stabilized by citrate (Cat \# 741949 from Aldrich) were both purchased from commercial vendors. Thus the risk associated with synthesis of these particles as well as from the waste is null for customers. However, the risk at manufacturing units cannot be assessed due to lack of knowledge of the protocols followed. The information sheets provided with the products do not mention any potential risk on usage.

\section{Formulation}

The $1.4 \mathrm{~nm}$ AuNPs are supplied in lyophilized form whereas $5 \mathrm{~nm}$ AuNPs are supplied in a colloidal suspension. $1.4 \mathrm{~nm}$ AuNPs need to be reconstituted in Milli-Q water 
before use. Type of formulation (aerosol, solution, or bulk solid) critically influences the susceptibility to exposure via inhalation, cutaneous contact, or ingestion. Potential risk of exposure, in a laboratory setting, based on formulation follows the order:

\section{Aerosol $>$ Solution $>$ Bulk solid}

Thus the risk of exposure to $1.4 \mathrm{~nm}$ AuNPs is slightly less than for $5 \mathrm{~nm}$ AuNPs till they are reconstituted. In practice the reconstitution step is less than 1 minute in duration. All solutions of AuNPs and the procedures involving AuNPs are carried out while wearing nitrile gloves (PPE class III) and a lab coat for protection against cutaneous exposure and contact with clothes. Overall, in solution based formulation there is low risk of exposure to 1.4 and $5 \mathrm{~nm}$ AuNPs, when handled with caution.

\section{Experimental work}

Labeling of the fibrils with AuNPs is carried out on the lab bench. The work area is spread with a filter paper to absorb accidental spillage. Lab coat and nitrile gloves are worn throughout the experiment. The waste from the experiments i.e. absorbent filter paper, gloves, tips, microfuge tubes, wipes etc. are discarded in the incinerator waste at the end of the experiment. Exposure to AuNPs during experiments following the above mentioned precautions is assumed to be low.

\section{Waste disposal}

The aSyn fibril waste with AuNPs after experiments is discarded in the incinerator waste. The solution waste from the experiment is collected in air tight plastic tubes and stored separately till further disposal. The present regimen for disposal includes incineration of all materials containing AuNPs waste. The residual solutions however have a lot of gold particles and are being stored with the intention to be able to recycle the metal therein. Thus exposure via the environment using the above mentioned waste disposal method is minimal.

\section{Stability}

The stability of the bio-based nanowires against leaching out of the nanoparticles from the assemblies is not known, but it is expected to be extremely low. The key step in attaining electronically conductive surface on the fibrils includes electroless chemical 
gold deposition on the smaller AuNPs (1.4 and $5 \mathrm{~nm}$ ). In this process individual gold nanoparticles increase to about 6-7 $\mathrm{nm}$ in diameter and are no longer separate particles but form a continuous sheet of gold on the surface of the fibril.

\section{Anticipated exposure and nanotoxicity by large scale production and use of bio-based nanowires}

In the event of scaling up of present protocols for production of bio-based nanowires on a large scale, safe and sustainable products can be manufactured with a few important precautions. Firstly, the whole process of fabricating the bio-based nanowires can be automated with the use of liquid handling systems. This would reduce the personnel level exposure to AuNPs during production to negligible levels. Secondly, methods should be developed to recycle the solution waste from manufacturing steps to avoid loss of valuable metal and to prevent pollution of air, water and soil (by present practices of waste disposal). Averting the release of AuNPs in the environment (air, water and soil) can reduce the risk of exposure to AuNPs via the environment to a minimum. Finally, with respect to the disposal of products containing the bio-based nanowires after use, buyback policies should be implemented to encourage recycling of materials and prevention of pollution.

\section{ACKNOWLEDGEMENTS}

We thank Dr. Adrienne J.A.M. Sips and Dr. Margriet V.D.Z. Park from National Institute for Public Health and the Environment (RIVM), The Netherlands, for their guidance on the risk evaluation of gold nanoparticles. 


\section{Abbreviations}

\begin{tabular}{|c|c|}
\hline aSyn & alpha-Synuclein \\
\hline$\beta$-lac & Beta-lactoglobulin \\
\hline $\mathrm{A} 30 \mathrm{P}$ & aSyn with alanine 30 mutated to proline \\
\hline A30P-A140C & $\begin{array}{l}\text { A30P protein sequence with residue } 140 \text { (alanine) substituted } \\
\text { for cysteine }\end{array}$ \\
\hline A53E & aSyn with alanine 53 mutated to glutamic acid \\
\hline A $53 \mathrm{~T}$ & aSyn with alanine 53 mutated to threonine \\
\hline A53T-A140C & $\begin{array}{l}\text { A53T protein sequence with residue } 140 \text { (alanine) substituted } \\
\text { for cysteine }\end{array}$ \\
\hline $\mathrm{AC}$ & Alternating current \\
\hline $\mathrm{AD}$ & Alzheimer's disease \\
\hline $\mathrm{ADF}-4$ & Araneus diadematus fibroin- 4 \\
\hline AF 488 & Alexa Fluor 488 \\
\hline AF 647 & Alexa Fluor 647 \\
\hline AFM & Atomic force microscopy \\
\hline ANS & 8-anilinonaphthalene-1-sulfonic acid \\
\hline APFO-12 & Alternating polyfluorene co-polymer-12 \\
\hline ATP & Adenosine triphosphate \\
\hline ATR-FTIR & $\begin{array}{l}\text { Attenuated total reflectance-Fourier transform infrared } \\
\text { spectroscopy }\end{array}$ \\
\hline AuNPs & Gold nanoparticles \\
\hline $\mathrm{A} \beta$ & Amyloid- $\beta$ \\
\hline BSE & Back scattered electrons \\
\hline $\mathrm{CD}$ & Circular dichroism \\
\hline CMA & Chaperone mediated autophagy \\
\hline $\mathrm{CMC}$ & Critical monomer concentration \\
\hline CPEs & Conjugated polyelectrolytes \\
\hline CR & Congo red \\
\hline $\mathrm{DC}$ & Direct current \\
\hline DCVJ & 9-(2,2-Dicyanovinyl) julolidine \\
\hline
\end{tabular}


DMSO Dimethyl sulfoxide

DTNB [5, 5'-dithiobis-(2-nitrobenzoic acid)]

DTT Dithiothreitol

E.coli BL21 (DE3) Competent Escherichia coli cells used for transformation and protein expression

E46K aSyn with glutamic acid 46 mutated to lysine

EDTA Ethylenediaminetetraacetic acid

EDX

EM

Energy-dispersive X-ray spectroscopy

Electron microscopy

EPR

Electron paramagnetic resonance

ETC

G51D

Electron transport chain

$\mathrm{G} \Omega$

aSyn with glycine 51 mutated to aspartic acid

Giga-ohms

H50Q

aSyn with histidine 50 mutated to glutamine

HAADF

High angle annular darkfield

HAM

Hierarchical assembly model

HRP

Horseradish peroxidase

IAPP

Islet amyloid polypeptide

ICD

Induced circular dichroism

IDPs

Intrinsically disordered proteins

IgG

Immunoglobulin $\mathrm{G}$

IPTG

Isopropyl $\beta$-D-1-thiogalactopyranoside

$\mathrm{kHz}$

Kilo-hertz

LBs

Lewy bodies

LB

Luria Bertani medium

LCOs

Luminescent conjugated oligothiophenes

LN

Lewy neurites

LRRK2

Leucine-rich repeat kinase-2

$\mathrm{mA}$

Milli-amperes

MicroED

Micro-electron diffraction

MicroIDTs

Micro-interdigitated electrodes

MsrA

Methionine sulfoxide reductase A

$\mathrm{mV}$

Milli-volts 
NAC

NACP

NMR

$\mathrm{nN}$

nPAGE

NPs

$\mathrm{NTB}^{2-}$

OD

$\mathrm{pA}$

PARK1

PARK4

PARK7

PBS

PcTS

PD

PD1

PDB

PEDOT/PSS

PEG

PFA

PINK1

PMSF

PONT 1

PONT-2

PPF

pT7-7

PVDF

RMC

ROS

STEM

SAXS
Non $A \beta$ amyloid component

Non $A \beta$ component of amyloid precursor

Nuclear magnetic resonance

Nano-newtons

Native polyacrylamide gel electrophoresis

Nano-particles

2-nitro-5-thiobenzoate

Optical density

Pico-amperes

Previous symbol for SNCA

Previous symbol for SNCA

Parkinson protein 7

Phosphate buffered saline

Phthalocyanine tetrasulphonate

Parkinson's disease

Synonym for SNCA

Protein data bank

Poly(3,4-ethylenedioxythiophene)/ polystyrene sulfonate

Poly ethylene glycol

Paraformaldehyde

PTEN induced putative kinase 1

Phenylmethylsulfonyl fluoride

$\operatorname{Poly}((3,3)$-di[(S)-5-amino-5-carbonyl-3-oxapentyl]-

[2,2';5',2"])-5,5"-terthiophenylene hydrochloride) 1

$\operatorname{Poly}((1,4-\operatorname{di}(3-[(S)-5$-amino-5-carbonyl-3-oxa-pentyl]-

thiophen-2-yl)-benzene) hydrochloride)

Poly(propylene fumarate)

Bacterial expression vector

Polyvinylidene difluoride

Residual monomer concentration

Reactive oxygen species

Scanning transmission electron microscopy

Small angle X-ray scattering 
SCC

SDS

SDS-PAGE

SE

SLAS

SNARE

SNCA

ssNMR

TBS-T

TCEP

TCSPC

TEM

ThS

ThT

UPS

Wt

Wt-A140C
Super critical concentration

Sodium dodecyl sulfate

Sodium dodecyl sulfate-polyacrylamide gel electrophoresis

Secondary electrons

Sodium lauroyl sarcosinate

Soluble N-ethylmaleimide-sensitive factor attachment protein receptor

Synuclein, alpha (non $\mathrm{A} \beta$ component of amyloid precursor)

Solid state NMR

Tris buffered saline-Tween

Tris (2-carboxyethyl) phosphine hydrochloride

Time correlated single photon counting

Transmission electron microscopy

Thioflavin-S

Thioflavin-T

Ubiquitin proteasome system

Wild type

Wild type aSyn sequence with residue 140 (alanine) substituted for cysteine 


\section{Bibliography}

Adamcik, J. and R. Mezzenga (2011). "Adjustable twisting periodic pitch of amyloid fibrils." Soft Matter 7(11): 5437-5443.

Adamcik, J., J. M. Jung, et al. (2010). "Understanding amyloid aggregation by statistical analysis of atomic force microscopy images." Nature Nanotechnology 5(6): 423-428.

Alessandrini, A. and P. Facci (2005). "AFM: a versatile tool in biophysics." Measurement Science \& Technology 16(6): R65-R92.

Andreasen, M., N. Lorenzen, et al. (2015). "Interactions between misfolded protein oligomers and membranes: A central topic in neurodegenerative diseases?" Biochimica et Biophysica Acta 1848(9): 1897-1907.

Andreu, J. M. and S. N. Timasheff (1986). "The measurement of cooperative protein selfassembly by turbidity and other techniques." Methods in Enzymology 130: 4759.

Apetri, M. M., N. C. Maiti, et al. (2006). "Secondary structure of alpha-synuclein oligomers: characterization by raman and atomic force microscopy." Journal of Molecular Biology 355(1): 63-71.

Arosio, P., T. P. Knowles, et al. (2015). "On the lag phase in amyloid fibril formation." Physical Chemistry Chemical Physics 17(12): 7606-7618.

Bai, H., K. Xu, et al. (2007). "Fabrication of Au nanowires of uniform length and diameter using a monodisperse and rigid biomolecular template: collagen-like triple helix." Angewandte Chemie. International Edition in English 46(18): 3319-3322.

Ban, T., M. Hoshino, et al. (2004). "Direct observation of Abeta amyloid fibril growth and inhibition." Journal of Molecular Biology 344(3): 757-767.

Banerjee, I. A., L. Yu, et al. (2003). "Cu nanocrystal growth on peptide nanotubes by biomineralization: size control of $\mathrm{Cu}$ nanocrystals by tuning peptide conformation." Proceedings of the National Academy of Sciences of the United States of America 100(25): 14678-14682.

Bartels, T., J. G. Choi, et al. (2011). "alpha-Synuclein occurs physiologically as a helically folded tetramer that resists aggregation." Nature 477(7362): 107-110.

Bartolini, M., C. Bertucci, et al. (2007). "Insight into the kinetic of amyloid beta (1-42) peptide self-aggregation: elucidation of inhibitors' mechanism of action." Chembiochem 8(17): 2152-2161. 
Bellino, M. G., E. J. Calvo, et al. (2004). "Adsorption kinetics of charged thiols on gold nanoparticles." Physical Chemistry Chemical Physics 6(2): 424-428.

Bendor, J. T., T. P. Logan, et al. (2013). "The function of alpha-synuclein." Neuron 79(6): 1044-1066.

Beyer, K., M. Domingo-Sabat, et al. (2008). "Identification and characterization of a new alpha-synuclein isoform and its role in Lewy body diseases." Neurogenetics 9(1): 15-23.

Biancalana, M. and S. Koide (2010). "Molecular mechanism of Thioflavin-T binding to amyloid fibrils." Biochimica et Biophysica Acta 1804(7): 1405-1412.

Bieschke, J., J. Russ, et al. (2010). "EGCG remodels mature alpha-synuclein and amyloidbeta fibrils and reduces cellular toxicity." Proceedings of the National Academy of Sciences of the United States of America 107(17): 7710-7715.

Bjork, P., A. Herland, et al. (2010). "Biomolecular nanowires decorated by organic electronic polymers." Journal of Materials Chemistry 20(12): 2269-2276.

Bongiovanni, M. N., D. B. Scanlon, et al. (2011). "Functional fibrils derived from the peptide TTR1-cycloRGDfK that target cell adhesion and spreading." Biomaterials 32(26): 6099-6110.

Bousset, L., L. Pieri, et al. (2013). "Structural and functional characterization of two alpha-synuclein strains." Nature Communications 4: 2575.

Breydo, L., J. W. Wu, et al. (2012). "Alpha-synuclein misfolding and Parkinson's disease." Biochimica et Biophysica Acta 1822(2): 261-285.

Breydo, L., K. D. Reddy, et al. (2014). "The crowd you're in with: effects of different types of crowding agents on protein aggregation." Biochimica et Biophysica Acta 1844(2): 346-357.

Buell, A. K., C. Galvagnion, et al. (2014). "Solution conditions determine the relative importance of nucleation and growth processes in alpha-synuclein aggregation." Proceedings of the National Academy of Sciences of the United States of America 111(21): 7671-7676.

Buell, A. K., P. Hung, et al. (2013). "Electrostatic effects in filamentous protein aggregation." Biophysical Journal 104(5): 1116-1126.

Burre, J., M. Sharma, et al. (2010). "Alpha-synuclein promotes SNARE-complex assembly in vivo and in vitro." Science 329(5999): 1663-1667.

Bush, A. I., W. H. Pettingell, et al. (1994). "Rapid induction of Alzheimer A beta amyloid formation by zinc." Science 265(5177): 1464-1467.

Bussell, R., Jr. and D. Eliezer (2003). "A structural and functional role for 11-mer repeats in alpha-synuclein and other exchangeable lipid binding proteins." Journal of Molecular Biology 329(4): 763-778. 
Campion, D., C. Martin, et al. (1995). "The NACP/synuclein gene: chromosomal assignment and screening for alterations in Alzheimer disease." Genomics 26(2): 254-257.

Campioni, S., G. Carret, et al. (2014). "The Presence of an Air-Water Interface Affects Formation and Elongation of alpha-Synuclein Fibrils." Journal of the American Chemical Society 136(7): 2866-2875.

Camponeschi, F., D. Valensin, et al. (2013). "Copper(I)-alpha-synuclein interaction: structural description of two independent and competing metal binding sites." Inorganic Chemistry 52(3): 1358-1367.

Capstick, D. S., A. Jomaa, et al. (2011). "Dual amyloid domains promote differential functioning of the chaplin proteins during Streptomyces aerial morphogenesis." Proceedings of the National Academy of Sciences of the United States of America 108(24): 9821-9826.

Carulla, N., G. L. Caddy, et al. (2005). "Molecular recycling within amyloid fibrils." Nature 436(7050): 554-558.

Celej, M. S., R. Sarroukh, et al. (2012). "Toxic prefibrillar alpha-synuclein amyloid oligomers adopt a distinctive antiparallel beta-sheet structure." Biochemical Journal 443(3): 719-726.

Cerf, E., R. Sarroukh, et al. (2009). "Antiparallel beta-sheet: a signature structure of the oligomeric amyloid beta-peptide." Biochemical Journal 421(3): 415-423.

Charrier, J. G. and C. Anastasio (2012). "On dithiothreitol (DTT) as a measure of oxidative potential for ambient particles: evidence for the importance of soluble transition metals." Atmospheric Chemistry and Physics 12(5): 11317-11350.

Chartier-Harlin, M. C., J. Kachergus, et al. (2004). "Alpha-synuclein locus duplication as a cause of familial Parkinson's disease." Lancet 364(9440): 1167-1169.

Chen, C. L. and N. L. Rosi (2010). "Peptide-based methods for the preparation of nanostructured inorganic materials." Angewandte Chemie. International Edition in English 49(11): 1924-1942.

Chen, M., M. Margittai, et al. (2007). "Investigation of alpha-synuclein fibril structure by site-directed spin labeling." Journal of Biological Chemistry 282(34): 2497024979.

Chen, S. W., S. Drakulic, et al. (2015). "Structural characterization of toxic oligomers that are kinetically trapped during alpha-synuclein fibril formation." Proceedings of the National Academy of Sciences of the United States of America 112(16): E1994-2003.

Chen, Y. S., Y. C. Hung, et al. (2009). "Assessment of the In Vivo Toxicity of Gold Nanoparticles." Nanoscale Research Letters 4(8): 858-864. 
Cherny, I. and E. Gazit (2008). "Amyloids: not only pathological agents but also ordered nanomaterials." Angewandte Chemie. International Edition in English 47(22): 4062-4069.

Chiti, F. and C. M. Dobson (2006). "Protein misfolding, functional amyloid, and human disease." Annual Review of Biochemistry 75: 333-366.

Chiti, F., M. Stefani, et al. (2003). "Rationalization of the effects of mutations on peptide and protein aggregation rates." Nature 424(6950): 805-808.

Chiti, F., N. Taddei, et al. (2002). "Kinetic partitioning of protein folding and aggregation." Nature Structural Biology 9(2): 137-143.

Choi, W., S. Zibaee, et al. (2004). "Mutation E46K increases phospholipid binding and assembly into filaments of human alpha-synuclein." FEBS Letters 576(3): 363368.

Choi, Y. S., J. Kim, et al. (2011). "Photoelectric Protein Nanofibrils of alpha-Synuclein with Embedded Iron and Phthalocyanine Tetrasulfonate." Angewandte Chemie-International Edition 50(27): 6070-6074.

Ciryam, P., R. Kundra, et al. (2015). "Supersaturation is a major driving force for protein aggregation in neurodegenerative diseases." Trends in Pharmacological Sciences 36(2): 72-77.

Clayton, D. F. and J. M. George (1998). "The synucleins: a family of proteins involved in synaptic function, plasticity, neurodegeneration and disease." Trends in Neurosciences 21(6): 249-254.

Cohen, S. I., M. Vendruscolo, et al. (2012). "From macroscopic measurements to microscopic mechanisms of protein aggregation." Journal of Molecular Biology 421(2-3): 160-171.

Cohen, S. I., S. Linse, et al. (2013). "Proliferation of amyloid-beta42 aggregates occurs through a secondary nucleation mechanism." Proceedings of the National Academy of Sciences of the United States of America 110(24): 9758-9763.

Colby, D. W. and S. B. Prusiner (2011). "Prions." Cold Spring Harbour Perspective Biology 3(1): a006833.

Colby, R., J. Hulleman, et al. (2008). "Biotemplated synthesis of metallic nanoparticle chains on an alpha-synuclein fiber scaffold." Journal of Nanoscience and Nanotechnology 8(2): 973-978.

Collinge, J. (2001). "Prion diseases of humans and animals: their causes and molecular basis." Annual Review of Neuroscience 24: 519-550.

Comellas, G., L. R. Lemkau, et al. (2011). "Structured regions of alpha-synuclein fibrils include the early-onset Parkinson's disease mutation sites." Journal of Molecular Biology 411(4): 881-895. 
Conway, K. A., J. D. Harper, et al. (1998). "Accelerated in vitro fibril formation by a mutant alpha-synuclein linked to early-onset Parkinson disease." Nature Medicine 4(11): 1318-1320.

Conway, K. A., J. D. Harper, et al. (2000). "Fibrils formed in vitro from alpha-synuclein and two mutant forms linked to Parkinson's disease are typical amyloid." Biochemistry 39(10): 2552-2563.

Conway, K. A., S. J. Lee, et al. (2000). "Acceleration of oligomerization, not fibrillization, is a shared property of both alpha-synuclein mutations linked to early-onset Parkinson's disease: implications for pathogenesis and therapy." Proceedings of the National Academy of Sciences of the United States of America 97(2): 571576.

Cuajungco, M. P., L. E. Goldstein, et al. (2000). "Evidence that the beta-amyloid plaques of Alzheimer's disease represent the redox-silencing and entombment of abeta by zinc." Journal of Biological Chemistry 275(26): 19439-19442.

Cuervo, A. M., L. Stefanis, et al. (2004). "Impaired degradation of mutant alphasynuclein by chaperone-mediated autophagy." Science 305(5688): 1292-1295.

Davidson, W. S., A. Jonas, et al. (1998). "Stabilization of alpha-synuclein secondary structure upon binding to synthetic membranes." Journal of Biological Chemistry 273(16): 9443-9449.

De Genst, E. J., T. Guilliams, et al. (2010). "Structure and properties of a complex of alpha-synuclein and a single-domain camelid antibody." Journal of Molecular Biology 402(2): 326-343.

De Jong, W. H., W. I. Hagens, et al. (2008). "Particle size-dependent organ distribution of gold nanoparticles after intravenous administration." Biomaterials 29(12): 1912-1919.

Dedmon, M. M., K. Lindorff-Larsen, et al. (2005). "Mapping long-range interactions in alpha-synuclein using spin-label NMR and ensemble molecular dynamics simulations." Journal of the American Chemical Society 127(2): 476-477.

Del Mar, C., E. A. Greenbaum, et al. (2005). "Structure and properties of alpha-synuclein and other amyloids determined at the amino acid level." Proceedings of the National Academy of Sciences of the United States of America 102(43): 1547715482.

Deleersnijder, A., M. Gerard, et al. (2013). "The remarkable conformational plasticity of alpha-synuclein: blessing or curse?" Trends in Molecular Medicine 19(6): 368377.

Devi, L., V. Raghavendran, et al. (2008). "Mitochondrial import and accumulation of alpha-synuclein impair complex I in human dopaminergic neuronal cultures 
and Parkinson disease brain." Journal of Biological Chemistry 283(14): 90899100.

Dickinson, A. G., H. Fraser, et al. (1975). "Scrapie incubation time can exceed natural lifespan." Nature 256(5520): 732-733.

Dill, K. A. and H. S. Chan (1997). "From Levinthal to pathways to funnels." Nature Structural Biology 4(1): 10-19.

Dobson, C. M. (2003). "Protein folding and misfolding." Nature 426(6968): 884-890.

Doria, G., J. Conde, et al. (2012). "Noble metal nanoparticles for biosensing applications." Sensors (Basel) 12(2): 1657-1687.

Dreaden, E. C., A. M. Alkilany, et al. (2012). "The golden age: gold nanoparticles for biomedicine." Chemical Society Reviews 41(7): 2740-2779.

Dunker, A. K., C. J. Brown, et al. (2002). "Intrinsic disorder and protein function." Biochemistry 41(21): 6573-6582.

Dunning, C. J., S. George, et al. (2013). "What's to like about the prion-like hypothesis for the spreading of aggregated alpha-synuclein in Parkinson disease?" Prion 7(1): 92-97.

Dzwolak, W. and M. Pecul (2005). "Chiral bias of amyloid fibrils revealed by the twisted conformation of Thioflavin T: an induced circular dichroism/DFT study." FEBS Lett 579(29): 6601-6603.

Eaton, W. A., P. A. Thompson, et al. (1996). "Fast events in protein folding." Structure 4(10): 1133-1139.

Ebrahimi-Fakhari, D., I. Cantuti-Castelvetri, et al. (2011). "Distinct roles in vivo for the ubiquitin-proteasome system and the autophagy-lysosomal pathway in the degradation of alpha-synuclein." Journal of Neuroscience 31(41): 14508-14520.

Eisenberg, D. and M. Jucker (2012). "The amyloid state of proteins in human diseases." Cell 148(6): 1188-1203.

Elghetany, M. T., A. Saleem, et al. (1989). "The congo red stain revisited." Annals of Clinical Laboratory Science 19(3): 190-195.

Eliezer, D., E. Kutluay, et al. (2001). "Conformational properties of alpha-synuclein in its free and lipid-associated states." Journal of Molecular Biology 307(4): 10611073.

Elliot, M. A. and N. J. Talbot (2004). "Building filaments in the air: aerial morphogenesis in bacteria and fungi." Current Opinion in Microbiology 7(6): 594-601.

Esler, W. P., E. R. Stimson, et al. (2000). "Alzheimer's disease amyloid propagation by a template-dependent dock-lock mechanism." Biochemistry 39(21): 6288-6295.

Faas, F. G., B. Rieger, et al. (2009). "DNA deformations near charged surfaces: electron and atomic force microscopy views." Biophysical Journal 97(4): 1148-1157. 
Fabian, H., K. Gast, et al. (2008). "Early stages of misfolding and association of beta2microglobulin: insights from infrared spectroscopy and dynamic light scattering." Biochemistry 47(26): 6895-6906.

Fandrich, M. (2012). "Oligomeric intermediates in amyloid formation: structure determination and mechanisms of toxicity." Journal of Molecular Biology 421(4-5): 427-440.

Fandrich, M., J. Meinhardt, et al. (2009). "Structural polymorphism of Alzheimer Abeta and other amyloid fibrils." Prion 3(2): 89-93.

Ferkinghoff-Borg, J., J. Fonslet, et al. (2010). "Stop-and-go kinetics in amyloid fibrillation." Physical Review. E, Statistical, Nonlinear, and Soft Matter Physics 82(1 Pt 1): 010901.

Ferrone, F. A., J. Hofrichter, et al. (1980). "Kinetic studies on photolysis-induced gelation of sickle cell hemoglobin suggest a new mechanism." Biophysical Journal 32(1): 361-380.

Ferrone, F. A., J. Hofrichter, et al. (1985). "Kinetics of sickle hemoglobin polymerization. II. A double nucleation mechanism." Journal of Molecular Biology 183(4): 611631.

Fink, A. L. (2005). "Natively unfolded proteins." Current Opinion in Structural Biology 15(1): 35-41.

Fink, A. L. (2006). "The aggregation and fibrillation of alpha-synuclein." Accounts of Chemical Research 39(9): 628-634.

Fitzpatrick, A. W., G. T. Debelouchina, et al. (2013). "Atomic structure and hierarchical assembly of a cross-beta amyloid fibril." Proceedings of the National Academy of Sciences of the United States of America 110(14): 5468-5473.

Fodera, V., F. Librizzi, et al. (2008). "Secondary nucleation and accessible surface in insulin amyloid fibril formation." Journal of Physical Chemistry B 112(12): 3853-3858.

Frieden, C. (2007). "Protein aggregation processes: In search of the mechanism." Protein Science 16(11): 2334-2344.

Fujiwara, S., F. Matsumoto, et al. (2003). "Effects of salt concentration on association of the amyloid protofilaments of hen egg white lysozyme studied by time-resolved neutron scattering." Journal of Molecular Biology 331(1): 21-28.

Garcia-Reitbock, P., O. Anichtchik, et al. (2010). "SNARE protein redistribution and synaptic failure in a transgenic mouse model of Parkinson's disease." Brain 133(Pt 7): 2032-2044.

Gath, J., L. Bousset, et al. (2014). "Unlike Twins: An NMR Comparison of Two alphaSynuclein Polymorphs Featuring Different Toxicity." PLoS ONE 9(3): e90659. 
Gazit, E. (2002). "A possible role for pi-stacking in the self-assembly of amyloid fibrils." FASEB Journal 16(1): 77-83.

Gazit, E. (2007). "Self-assembled peptide nanostructures: the design of molecular building blocks and their technological utilization." Chemical Society Reviews 36(8): 1263-1269.

Gazit, E. (2007). "Use of biomolecular templates for the fabrication of metal nanowires." FEBS Journal 274(2): 317-322.

George, J. M. (2002). "The synucleins." Genome Biology 3(1): REVIEWS3002.

George, J. M., H. Jin, et al. (1995). "Characterization of a novel protein regulated during the critical period for song learning in the zebra finch." Neuron 15(2): 361-372.

George, S., N. L. Rey, et al. (2013). "alpha-Synuclein: the long distance runner." Brain Pathology 23(3): 350-357.

Georgieva, E. R., T. F. Ramlall, et al. (2008). "Membrane-bound alpha-synuclein forms an extended helix: long-distance pulsed ESR measurements using vesicles, bicelles, and rodlike micelles." Journal of the American Chemical Society 130(39): 12856-12857.

Gershenson, A., L. M. Gierasch, et al. (2014). "Energy landscapes of functional proteins are inherently risky." Nature Chemical Biology 10(11): 884-891.

Ghosh, D., M. Mondal, et al. (2013). "The Parkinson's disease-associated H50Q mutation accelerates alpha-Synuclein aggregation in vitro." Biochemistry 52(40): 69256927.

Ghosh, D., S. Sahay, et al. (2014). "The newly discovered Parkinson's disease associated Finnish mutation (A53E) attenuates alpha-synuclein aggregation and membrane binding." Biochemistry 53(41): 6419-6421.

Giasson, B. I., I. V. Murray, et al. (2001). "A hydrophobic stretch of 12 amino acid residues in the middle of alpha-synuclein is essential for filament assembly." Journal of Biological Chemistry 276(4): 2380-2386.

Giasson, B. I., K. Uryu, et al. (1999). "Mutant and wild type human alpha-synucleins assemble into elongated filaments with distinct morphologies in vitro." Journal of Biological Chemistry 274(12): 7619-7622.

Giasson, B. I., M. S. Forman, et al. (2003). "Initiation and synergistic fibrillization of tau and alpha-synuclein." Science 300(5619): 636-640.

Giehm, L., D. I. Svergun, et al. (2011). "Low-resolution structure of a vesicle disrupting \&alpha;-synuclein oligomer that accumulates during fibrillation." Proceedings of the National Academy of Sciences of the United States of America 108(8): 3246-3251. 
Giehm, L., N. Lorenzen, et al. (2011). "Assays for alpha-synuclein aggregation." Methods 53(3): 295-305.

Goedert, M. (2015). "NEURODEGENERATION. Alzheimer's and Parkinson's diseases: The prion concept in relation to assembled Abeta, tau, and alpha-synuclein." Science 349(6248): 1255555.

Goedert, M., B. Falcon, et al. (2014). "Prion-like mechanisms in the pathogenesis of tauopathies and synucleinopathies." Current Neurology and Neuroscience Reports 14(11): 495.

Goedert, M., M. G. Spillantini, et al. (2013). "100 years of Lewy pathology." Nature Reviews Neurology 9(1): 13-24.

Goldsbury, C., J. Kistler, et al. (1999). "Watching amyloid fibrils grow by time-lapse atomic force microscopy." Journal of Molecular Biology 285(1): 33-39.

Goldsbury, C., P. Frey, et al. (2005). "Multiple assembly pathways underlie amyloid-beta fibril polymorphisms." Journal of Molecular Biology 352(2): 282-298.

Goormaghtigh, E., V. Raussens, et al. (1999). "Attenuated total reflection infrared spectroscopy of proteins and lipids in biological membranes." Biochimica et Biophysica Acta 1422(2): 105-185.

Gorelsky, S. I., L. Basumallick, et al. (2005). "Spectroscopic and DFT investigation of [M\{HB(3,5-iPr2pz)3\}(SC6F5)] ( $=\mathrm{Mn}, \mathrm{Fe}, \mathrm{Co}, \mathrm{Ni}, \mathrm{Cu}$, and $\mathrm{Zn})$ model complexes: periodic trends in metal-thiolate bonding." Inorganic Chemistry 44(14): 4947-4960.

Gosal, W. S., I. J. Morten, et al. (2005). "Competing pathways determine fibril morphology in the self-assembly of beta2-microglobulin into amyloid." Journal of Molecular Biology 351(4): 850-864.

Gras, S. L., A. K. Tickler, et al. (2008). "Functionalised amyloid fibrils for roles in cell adhesion." Biomaterials 29(11): 1553-1562.

Greenbaum, E. A., C. L. Graves, et al. (2005). "The E46K mutation in alpha-synuclein increases amyloid fibril formation." Journal of Biological Chemistry 280(9): 7800-7807.

Grimsley, G. R., Pace, C. N. (2004). Spectrophotometric Determination of Protein Concentration. Current Protocols in Protein Science. 33: 3.1.1-3.1.9.

Groenning, M. (2010). "Binding mode of Thioflavin T and other molecular probes in the context of amyloid fibrils-current status." Journal of Chemical Biology 3(1): 118.

Gruschus, J. M., T. L. Yap, et al. (2013). "NMR structure of calmodulin complexed to an N-terminally acetylated alpha-synuclein peptide." Biochemistry 52(20): 34363445 . 
Guilliams, T., F. El-Turk, et al. (2013). "Nanobodies raised against monomeric alphasynuclein distinguish between fibrils at different maturation stages." Journal of Molecular Biology 425(14): 2397-2411.

Gunasekaran, K., C. J. Tsai, et al. (2003). "Extended disordered proteins: targeting function with less scaffold." Trends in Biochemical Sciences 28(2): 81-85.

Guo, J. L., D. J. Covell, et al. (2013). "Distinct alpha-synuclein strains differentially promote tau inclusions in neurons." Cell 154(1): 103-117.

Guo, Z. Y. and D. Thirumalai (1995). "Kinetics of Protein-Folding - Nucleation Mechanism, Time Scales, and Pathways." Biopolymers 36(1): 83-102.

Haass, C. and D. J. Selkoe (2007). "Soluble protein oligomers in neurodegeneration: lessons from the Alzheimer's amyloid beta-peptide." Nature Reviews. Molecular Cell Biology 8(2): 101-112.

Hamada, D., I. Yanagihara, et al. (2004). "Engineering amyloidogenicity towards the development of nanofibrillar materials." Trends in Biotechnology 22(2): 93-97.

Hamedi, M., A. Herland, et al. (2008). "Electrochemical devices made from conducting nanowire networks self-assembled from amyloid fibrils and alkoxysulfonate PEDOT." Nano Letters 8(6): 1736-1740.

Hamodrakas, S. J., A. Hoenger, et al. (2004). "Amyloid fibrillogenesis of silkmoth chorion protein peptide-analogues via a liquid-crystalline intermediate phase." Journal of Structural Biology 145(3): 226-235.

Harper, J. D. and P. T. Lansbury, Jr. (1997). "Models of amyloid seeding in Alzheimer's disease and scrapie: mechanistic truths and physiological consequences of the time-dependent solubility of amyloid proteins." Annual Review of Biochemistry 66: 385-407.

Harper, J. D., S. S. Wong, et al. (1999). "Assembly of A beta amyloid protofibrils: an in vitro model for a possible early event in Alzheimer's disease." Biochemistry 38(28): 8972-8980.

Heilemann, M., P. Tinnefeld, et al. (2004). "Multistep energy transfer in single molecular photonic wires." Journal of the American Chemical Society 126(21): 6514-6515.

Heise, H., M. S. Celej, et al. (2008). "Solid-state NMR reveals structural differences between fibrils of wild-type and disease-related A53T mutant alpha-synuclein." Journal of Molecular Biology 380(3): 444-450.

Heise, H., W. Hoyer, et al. (2005). "Molecular-level secondary structure, polymorphism, and dynamics of full-length alpha-synuclein fibrils studied by solid-state NMR." Proceedings of the National Academy of Sciences of the United States of America 102(44): 15871-15876. 
Heldt, C. L., S. Zhang, et al. (2011). "Asymmetric amyloid fibril elongation: a new perspective on a symmetric world." Proteins 79(1): 92-98.

Herland, A., D. Thomsson, et al. (2008). "Decoration of amyloid fibrils with luminescent conjugated polymers." Journal of Materials Chemistry 18(1): 126-132.

Herland, A., P. Bjork, et al. (2005). "Electroactive luminescent self-assembled bioorganic nanowires: Integration of semiconducting oligoelectrolytes within amyloidogenic proteins (vol 17, pg 1466, 2005)." Advanced Materials 17(14): 1703-1703.

Herrera, F. E., A. Chesi, et al. (2008). "Inhibition of alpha-synuclein fibrillization by dopamine is mediated by interactions with five C-terminal residues and with E83 in the NAC region." PLoS ONE 3(10): e3394.

Herva, M. E., S. Zibaee, et al. (2014). "Anti-amyloid compounds inhibit alpha-synuclein aggregation induced by protein misfolding cyclic amplification (PMCA)." Journal of Biological Chemistry 289(17): 11897-11905.

Hilker, R., J. M. Brotchie, et al. (2011). "Pros and cons of a prion-like pathogenesis in Parkinson's disease." BMC Neurology 11: 74.

Holmqvist, S., O. Chutna, et al. (2014). "Direct evidence of Parkinson pathology spread from the gastrointestinal tract to the brain in rats." Acta Neuropathologica 128(6): 805-820.

Hong, D. P., S. Han, et al. (2011). "Characterization of the non-fibrillar alpha-synuclein oligomers." Protein and Peptide Letters 18(3): 230-240.

Horii, A., X. Wang, et al. (2007). "Biological designer self-assembling peptide nanofiber scaffolds significantly enhance osteoblast proliferation, differentiation and 3-D migration." PLoS ONE 2(2): e190.

Hoyer, W., D. Cherny, et al. (2004). "Impact of the acidic C-terminal region comprising amino acids 109-140 on alpha-synuclein aggregation in vitro." Biochemistry 43(51): 16233-16242.

Hoyer, W., D. Cherny, et al. (2004). "Rapid self-assembly of alpha-synuclein observed by in situ atomic force microscopy." Journal of Molecular Biology 340(1): 127-139.

Hoyer, W., T. Antony, et al. (2002). "Dependence of alpha-synuclein aggregate morphology on solution conditions." Journal of Molecular Biology 322(2): 383393.

Ikenoue, T., Y. H. Lee, et al. (2014). "Cold Denaturation of alpha-Synuclein Amyloid Fibrils." Angewandte Chemie. International Edition in English 53(30): 77997804. 
Ionescu-Zanetti, C., R. Khurana, et al. (1999). "Monitoring the assembly of Ig light-chain amyloid fibrils by atomic force microscopy." Proceedings of the National Academy of Sciences of the United States of America 96(23): 13175-13179.

Jahn, T. R., M. J. Parker, et al. (2006). "Amyloid formation under physiological conditions proceeds via a native-like folding intermediate." Nature Structural and Molecular Biology 13(3): 195-201.

Jarrett, J. T. and P. T. Lansbury, Jr. (1993). "Seeding "one-dimensional crystallization" of amyloid: a pathogenic mechanism in Alzheimer's disease and scrapie?" Cell 73(6): 1055-1058.

Jimenez, J. L., E. J. Nettleton, et al. (2002). "The protofilament structure of insulin amyloid fibrils." Proceedings of the National Academy of Sciences of the United States of America 99(14): 9196-9201.

Jucker, M. and L. C. Walker (2013). "Self-propagation of pathogenic protein aggregates in neurodegenerative diseases." Nature 501(7465): 45-51.

Kalia, L. V., S. K. Kalia, et al. (2013). "alpha-Synuclein oligomers and clinical implications for Parkinson disease." Annals of Neurology 73(2): 155-169.

Kanduri, C., T. Kuusi, et al. (2015). "The effect of music performance on the transcriptome of professional musicians." Science Reports 5: 9506.

Kasai, S., Y. Ohga, et al. (2004). "Multifunctional peptide fibrils for biomedical materials." Biopolymers 76(1): 27-33.

Kayed, R., E. Head, et al. (2003). "Common structure of soluble amyloid oligomers implies common mechanism of pathogenesis." Science 300(5618): 486-489.

Kellermayer, M. S., A. Karsai, et al. (2008). "Stepwise dynamics of epitaxially growing single amyloid fibrils." Proceedings of the National Academy of Sciences of the United States of America 105(1): 141-144.

Khurana, R., C. Ionescu-Zanetti, et al. (2003). "A general model for amyloid fibril assembly based on morphological studies using atomic force microscopy." Biophysical Journal 85(2): 1135-1144.

Klingstedt, T., C. Blechschmidt, et al. (2013). "Luminescent conjugated oligothiophenes for sensitive fluorescent assignment of protein inclusion bodies." Chembiochem 14(5): 607-616.

Knowles, T. P., C. A. Waudby, et al. (2009). "An analytical solution to the kinetics of breakable filament assembly." Science 326(5959): 1533-1537.

Knowles, T. P., M. Vendruscolo, et al. (2014). "The amyloid state and its association with protein misfolding diseases." Nature Reviews. Molecular Cell Biology 15(6): 384-396. 
Knowles, T. P., T. W. Oppenheim, et al. (2010). "Nanostructured films from hierarchical self-assembly of amyloidogenic proteins." Nature Nanotechnology 5(3): 204207.

Kodali, R. and R. Wetzel (2007). "Polymorphism in the intermediates and products of amyloid assembly." Current Opinion in Structural Biology 17(1): 48-57.

Kotzbauer, P. T., B. I. Giasson, et al. (2004). "Fibrillization of alpha-synuclein and tau in familial Parkinson's disease caused by the A53T alpha-synuclein mutation." Experimental Neurology 187(2): 279-288.

Kowaltowski, A. J., N. C. de Souza-Pinto, et al. (2009). "Mitochondria and reactive oxygen species." Free Radical Biology and Medicine 47(4): 333-343.

Kozlowski, H., M. Luczkowski, et al. (2012). "Copper, zinc and iron in neurodegenerative diseases (Alzheimer's, Parkinson's and prion diseases)." Coordination Chemistry Reviews 256(19-20): 2129-2141.

Krebs, M. R., C. E. Macphee, et al. (2004). "The formation of spherulites by amyloid fibrils of bovine insulin." Proceedings of the National Academy of Sciences of the United States of America 101(40): 14420-14424.

Krebs, M. R., E. H. Bromley, et al. (2005). "The binding of thioflavin-T to amyloid fibrils: localisation and implications." Journal of Structural Biology 149(1): 30-37.

Krebs, M. R., L. A. Morozova-Roche, et al. (2004). "Observation of sequence specificity in the seeding of protein amyloid fibrils." Protein Science 13(7): 1933-1938.

Kruger, R., W. Kuhn, et al. (1998). "Ala30Pro mutation in the gene encoding alphasynuclein in Parkinson's disease." Nature Genetics 18(2): 106-108.

Kuznetsova, I. M., B. Y. Zaslavsky, et al. (2015). "Beyond the excluded volume effects: mechanistic complexity of the crowded milieu." Molecules 20(1): 1377-1409.

Lansbury, P. T., Jr. (2004). "Back to the future: the 'old-fashioned' way to new medications for neurodegeneration." Nature Medicine 10 Suppl: S51-57.

Lara, C., J. Adamcik, et al. (2011). "General self-assembly mechanism converting hydrolyzed globular proteins into giant multistranded amyloid ribbons." Biomacromolecules 12(5): 1868-1875.

Lara, C., S. Gourdin-Bertin, et al. (2012). "Self-assembly of ovalbumin into amyloid and non-amyloid fibrils." Biomacromolecules 13(12): 4213-4221.

Lashuel, H. A. and J. S. Wall (2005). "Molecular electron microscopy approaches to elucidating the mechanisms of protein fibrillogenesis." Methods in Molecular Biology 299: 81-101.

Lashuel, H. A., B. M. Petre, et al. (2002). "Alpha-synuclein, especially the Parkinson's disease-associated mutants, forms pore-like annular and tubular protofibrils." Journal of Molecular Biology 322(5): 1089-1102. 
Lee, D., Y. J. Choe, et al. (2011). "Photoconductivity of pea-pod-type chains of gold nanoparticles encapsulated within dielectric amyloid protein nanofibrils of alpha-synuclein." Angewandte Chemie. International Edition in English 50(6): 1332-1337.

Lee, J. C., B. T. Lai, et al. (2007). "Alpha-synuclein tertiary contact dynamics." Journal of Physical Chemistry B 111(8): 2107-2112.

Lee, J. H., S. Kang, et al. (2010). "Fibrillar superstructure formation of hemoglobin A and its conductive, photodynamic and photovoltaic effects." Acta Biomaterialia 6(12): 4689-4697.

Legleiter, J. (2009). "The effect of drive frequency and set point amplitude on tapping forces in atomic force microscopy: simulation and experiment." Nanotechnology 20(24).

Lemkau, L. R., G. Comellas, et al. (2012). "Mutant protein A30P alpha-synuclein adopts wild-type fibril structure, despite slower fibrillation kinetics." Journal of Biological Chemistry 287(14): 11526-11532.

Lesage, S., M. Anheim, et al. (2013). "G51D alpha-synuclein mutation causes a novel parkinsonian-pyramidal syndrome." Annals of Neurology 73(4): 459-471.

LeVine, H., 3rd (1999). "Quantification of beta-sheet amyloid fibril structures with thioflavin T." Methods in Enzymology 309: 274-284.

Levy, R., N. T. Thanh, et al. (2004). "Rational and combinatorial design of peptide capping ligands for gold nanoparticles." Journal of the American Chemical Society 126(32): 10076-10084.

Li, C., A. K. Born, et al. (2014). "Amyloid-hydroxyapatite bone biomimetic composites." Advanced Materials 26(20): 3207-3212.

Li, C., J. Adamcik, et al. (2012). "Biodegradable nanocomposites of amyloid fibrils and graphene with shape-memory and enzyme-sensing properties." Nature Nanotechnology 7(7): 421-427.

Li, C., S. Bolisetty, et al. (2013). "Hybrid nanocomposites of gold single-crystal platelets and amyloid fibrils with tunable fluorescence, conductivity, and sensing properties." Advanced Materials 25(27): 3694-3700.

Li, D., E. M. Jones, et al. (2014). "Structure-based design of functional amyloid materials." Journal of the American Chemical Society 136(52): 18044-18051.

Li, J., V. N. Uversky, et al. (2001). "Effect of familial Parkinson's disease point mutations $\mathrm{A} 30 \mathrm{P}$ and $\mathrm{A} 53 \mathrm{~T}$ on the structural properties, aggregation, and fibrillation of human alpha-synuclein." Biochemistry 40(38): 11604-11613.

Lin, M. T. and M. F. Beal (2006). "Mitochondrial dysfunction and oxidative stress in neurodegenerative diseases." Nature 443(7113): 787-795. 
Lindberg, D. J., M. S. Wranne, et al. (2015). "Steady-state and time-resolved Thioflavin$\mathrm{T}$ fluorescence can report on morphological differences in amyloid fibrils formed by Abeta(1-40) and Abeta(1-42)." Biochemical and Biophysical Research Communications 458(2): 418-423.

Lindgren, M., K. Sorgjerd, et al. (2005). "Detection and characterization of aggregates, prefibrillar amyloidogenic oligomers, and protofibrils using fluorescence spectroscopy." Biophysical Journal 88(6): 4200-4212.

Liu, B., A. Moloney, et al. (2011). "Iron promotes the toxicity of amyloid beta peptide by impeding its ordered aggregation." Journal of Biological Chemistry 286(6): 4248-4256.

Liu, J. (2005). "Scanning transmission electron microscopy and its application to the study of nanoparticles and nanoparticle systems." Journal of Electron Microscopy 54(3): 251-278.

Liu, Y., W. Meyer-Zaika, et al. (2003). "Gold-cluster degradation by the transition of BDNA into A-DNA and the formation of nanowires." Angewandte Chemie. International Edition in English 42(25): 2853-2857.

Liu, Z., Y. Wu, et al. (2014). "Effects of internalized gold nanoparticles with respect to cytotoxicity and invasion activity in lung cancer cells." PLoS ONE 9(6): e99175. Lockhart, A., L. Ye, et al. (2005). "Evidence for the presence of three distinct binding sites for the thioflavin T class of Alzheimer's disease PET imaging agents on betaamyloid peptide fibrils." Journal of Biological Chemistry 280(9): 7677-7684.

Loksztejn, A. and W. Dzwolak (2008). "Chiral bifurcation in aggregating insulin: an induced circular dichroism study." Journal of Molecular Biology 379(1): 9-16.

Longair, M. H., D. A. Baker, et al. (2011). "Simple Neurite Tracer: open source software for reconstruction, visualization and analysis of neuronal processes." Bioinformatics 27(17): 2453-2454.

Lorenzen, N., S. B. Nielsen, et al. (2014). "The role of stable alpha-synuclein oligomers in the molecular events underlying amyloid formation." Journal of the American Chemical Society 136(10): 3859-3868.

Love, J. C., L. A. Estroff, et al. (2005). "Self-assembled monolayers of thiolates on metals as a form of nanotechnology." Chemical Reviews 105(4): 1103-1169.

Lu, J. H., M. T. Ardah, et al. (2011). "Baicalein inhibits formation of alpha-synuclein oligomers within living cells and prevents Abeta peptide fibrillation and oligomerisation." Chembiochem 12(4): 615-624.

Lu, J. X., W. Qiang, et al. (2013). "Molecular structure of beta-amyloid fibrils in Alzheimer's disease brain tissue." Cell 154(6): 1257-1268. 
Lu, T., Y. Pan, et al. (2004). "Gene regulation and DNA damage in the ageing human brain." Nature 429(6994): 883-891.

Luth, E. S., T. Bartels, et al. (2015). "Purification of alpha-Synuclein from Human Brain Reveals an Instability of Endogenous Multimers as the Protein Approaches Purity." Biochemistry 54(2): 279-292.

Ma, J., H. Komatsu, et al. (2013). "Intrinsic structural heterogeneity and long-term maturation of amyloid beta peptide fibrils." ACS Chemical Neuroscience 4(8): 1236-1243.

Maji, S. K., M. H. Perrin, et al. (2009). "Functional amyloids as natural storage of peptide hormones in pituitary secretory granules." Science 325(5938): 328-332.

Makarava, N. and I. V. Baskakov (2013). "The evolution of transmissible prions: the role of deformed templating." PLoS Pathogens 9(12): e1003759.

Maltsev, A. S., J. Chen, et al. (2013). "Site-specific interaction between alpha-synuclein and membranes probed by NMR-observed methionine oxidation rates." Journal of the American Chemical Society 135(8): 2943-2946.

Mankar, S., A. Anoop, et al. (2011). "Nanomaterials: amyloids reflect their brighter side." Nano Reviews 2.

Mann, S. (2009). "Self-assembly and transformation of hybrid nano-objects and nanostructures under equilibrium and non-equilibrium conditions." Nature Materials 8(10): 781-792.

Marek, P. J., V. Patsalo, et al. (2012). "Ionic strength effects on amyloid formation by amylin are a complicated interplay among Debye screening, ion selectivity, and Hofmeister effects." Biochemistry 51(43): 8478-8490.

Maroteaux, L., J. T. Campanelli, et al. (1988). "Synuclein: a neuron-specific protein localized to the nucleus and presynaptic nerve terminal." Journal of Neuroscience 8(8): 2804-2815.

Massi, F. and J. E. Straub (2001). "Energy landscape theory for Alzheimer's amyloid betapeptide fibril elongation." Proteins 42(2): 217-229.

Meersman, F. and C. M. Dobson (2006). "Probing the pressure-temperature stability of amyloid fibrils provides new insights into their molecular properties." Biochimica et Biophysica Acta 1764(3): 452-460.

Meisl, G., X. Yang, et al. (2014). "Differences in nucleation behavior underlie the contrasting aggregation kinetics of the Abeta40 and Abeta42 peptides." Proceedings of the National Academy of Sciences of the United States of America 111(26): 9384-9389. 
Miake, H., H. Mizusawa, et al. (2002). "Biochemical characterization of the core structure of alpha-synuclein filaments." Journal of Biological Chemistry 277(21): 1921319219.

Miller, G. and F. Wickson (2015). "Risk Analysis of Nanomaterials: Exposing Nanotechnology's Naked Emperor." Review of Policy Research 32(4): 485-512.

Miotto, M. C., E. E. Rodriguez, et al. (2014). "Site-specific copper-catalyzed oxidation of alpha-synuclein: tightening the link between metal binding and protein oxidative damage in Parkinson's disease." Inorganic Chemistry 53(9): 43504358.

Mirecka, E. A., H. Shaykhalishahi, et al. (2014). "Sequestration of a beta-hairpin for control of alpha-synuclein aggregation." Angewandte Chemie. International Edition in English 53(16): 4227-4230.

Morales, R., I. Moreno-Gonzalez, et al. (2013). "Cross-seeding of misfolded proteins: implications for etiology and pathogenesis of protein misfolding diseases." PLOS Pathogens 9(9): e1003537.

Morel, B., L. Varela, et al. (2010). "Environmental conditions affect the kinetics of nucleation of amyloid fibrils and determine their morphology." Biophysical Journal 99(11): 3801-3810.

Morris, V. J., A. R. Kirby, et al. (2010). Atomic force microscopy for biologists, Imperial College Press.

Muller, C., R. Jansson, et al. (2011). "Functionalisation of recombinant spider silk with conjugated polyelectrolytes." Journal of Materials Chemistry 21(9): 2909-2915.

Munishkina, L. A., E. M. Cooper, et al. (2004). "The effect of macromolecular crowding on protein aggregation and amyloid fibril formation." Journal of Molecular Recognition 17(5): 456-464.

Murray, I. V., B. I. Giasson, et al. (2003). "Role of alpha-synuclein carboxy-terminus on fibril formation in vitro." Biochemistry 42(28): 8530-8540.

Narhi, L., S. J. Wood, et al. (1999). "Both familial Parkinson's disease mutations accelerate alpha-synuclein aggregation." Journal of Biological Chemistry 274(14): 9843-9846.

Narkiewicz, J., G. Giachin, et al. (2014). "In vitro aggregation assays for the characterization of alpha-synuclein prion-like properties." Prion 8(1): 19-32.

Nath, A., M. Sammalkorpi, et al. (2012). "The conformational ensembles of alphasynuclein and tau: combining single-molecule FRET and simulations." Biophysical Journal 103(9): 1940-1949.

New, E. J. (2013). "Tools to study distinct metal pools in biology." Dalton Transactions 42(9): 3210-3219. 
Nichols, B. J. and H. R. Pelham (1998). "SNAREs and membrane fusion in the Golgi apparatus." Biochimica et Biophysica Acta 1404(1-2): 9-31.

Nielsen, L., R. Khurana, et al. (2001). "Effect of environmental factors on the kinetics of insulin fibril formation: elucidation of the molecular mechanism." Biochemistry 40(20): 6036-6046.

Nielsen, S. B., F. Macchi, et al. (2013). "Wildtype and A30P mutant alpha-synuclein form different fibril structures." PLoS ONE 8(7): e67713.

Nystrom, S., K. M. Psonka-Antonczyk, et al. (2013). "Evidence for age-dependent in vivo conformational rearrangement within Abeta amyloid deposits." ACS Chemical Biology 8(6): 1128-1133.

Ono, M. and H. Saji (2011). "SPECT Imaging Agents for Detecting Cerebral betaAmyloid Plaques." International Journal of Molecular Imaging 2011: 543267.

O'Nuallain, B., A. D. Williams, et al. (2004). "Seeding specificity in amyloid growth induced by heterologous fibrils." Journal of Biological Chemistry 279(17): 17490-17499.

Onuchic, J. N., Z. Luthey-Schulten, et al. (1997). "Theory of protein folding: the energy landscape perspective." Annual Review of Physical Chemistry 48: 545-600.

Ostrov, N. and E. Gazit (2010). "Genetic engineering of biomolecular scaffolds for the fabrication of organic and metallic nanowires." Angewandte Chemie. International Edition in English 49(17): 3018-3021.

Pan, Y., A. Leifert, et al. (2009). "Gold nanoparticles of diameter $1.4 \mathrm{~nm}$ trigger necrosis by oxidative stress and mitochondrial damage." Small 5(18): 2067-2076.

Paravastu, A. K., I. Qahwash, et al. (2009). "Seeded growth of beta-amyloid fibrils from Alzheimer's brain-derived fibrils produces a distinct fibril structure." Proceedings of the National Academy of Sciences of the United States of America 106(18): 7443-7448.

Paravastu, A. K., R. D. Leapman, et al. (2008). "Molecular structural basis for polymorphism in Alzheimer's beta-amyloid fibrils." Proceedings of the National Academy of Sciences of the United States of America 105(47): 1834918354.

Pasanen, P., L. Myllykangas, et al. (2014). "A novel alpha-synuclein mutation A53E associated with atypical multiple system atrophy and Parkinson's disease-type pathology." Neurobiology of Aging 35(9): 2180 e2181-2185.

Paslawski, W., M. Andreasen, et al. (2014). "High stability and cooperative unfolding of alpha-synuclein oligomers." Biochemistry 53(39): 6252-6263.

Paslawski, W., S. Mysling, et al. (2014). "Co-existence of two different alpha-synuclein oligomers with different core structures determined by hydrogen/deuterium 
exchange mass spectrometry." Angewandte Chemie. International Edition in English 53(29): 7560-7563.

Passos, J. F. and T. Von Zglinicki (2006). "Oxygen free radicals in cell senescence: are they signal transducers?" Free Radical Research 40(12): 1277-1283.

Patolsky, F., Y. Weizmann, et al. (2004). "Actin-based metallic nanowires as bionanotransporters." Nature Materials 3(10): 692-695.

Peelaerts, W., L. Bousset, et al. (2015). "alpha-Synuclein strains cause distinct synucleinopathies after local and systemic administration." Nature 522(7556): 340-344.

Pellarin, R., P. Schuetz, et al. (2010). "Amyloid fibril polymorphism is under kinetic control." Journal of the American Chemical Society 132(42): 14960-14970.

Pennycook, S. J., A. R. Lupini, et al. (2007). Scanning Transmission Electron Microscopy for Nanostructure Characterization. Scanning Microscopy for Nanotechnology. W. Zhou and Z. Wang, Springer New York: 152-191.

Perrett, R. M., Z. Alexopoulou, et al. (2015). "The endosomal pathway in Parkinson's disease." Molecular and Cellular Neurosciences 66(Pt A): 21-28.

Petkova, A. T., R. D. Leapman, et al. (2005). "Self-propagating, molecular-level polymorphism in Alzheimer's beta-amyloid fibrils." Science 307(5707): 262265.

Pinotsi, D., A. K. Buell, et al. (2014). "Direct observation of heterogeneous amyloid fibril growth kinetics via two-color super-resolution microscopy." Nano Letters 14(1): 339-345.

Plotegher, N., E. Greggio, et al. (2014). "Biophysical groundwork as a hinge to unravel the biology of alpha-synuclein aggregation and toxicity." Quarterly Reviews of Biophysics 47(1): 1-48.

Polymeropoulos, M. H., C. Lavedan, et al. (1997). "Mutation in the alpha-synuclein gene identified in families with Parkinson's disease." Science 276(5321): 2045-2047.

Powers, E. T. and D. L. Powers (2006). "The kinetics of nucleated polymerizations at high concentrations: amyloid fibril formation near and above the "supercritical concentration"." Biophysical Journal 91(1): 122-132.

Pranke, I. M., V. Morello, et al. (2011). "alpha-Synuclein and ALPS motifs are membrane curvature sensors whose contrasting chemistry mediates selective vesicle binding." Journal of Cell Biology 194(1): 89-103.

Pronchik, J., X. He, et al. (2010). "In vitro formation of amyloid from alpha-synuclein is dominated by reactions at hydrophobic interfaces." Journal of the American Chemical Society 132(28): 9797-9803. 
Proukakis, C., C. G. Dudzik, et al. (2013). "A novel alpha-synuclein missense mutation in Parkinson disease." Neurology 80(11): 1062-1064.

Prusiner, S. B. (1998). "Prions." Proceedings of the National Academy of Sciences of the United States of America 95(23): 13363-13383.

Pulawski, W., U. Ghoshdastider, et al. (2012). "Ubiquitous amyloids." Applied Biochemistry and Biotechnology 166(7): 1626-1643.

Qiang, W., K. Kelley, et al. (2013). "Polymorph-specific kinetics and thermodynamics of beta-amyloid fibril growth." Journal of the American Chemical Society 135(18): 6860-6871.

Qin, Z., D. Hu, et al. (2007). "Role of different regions of alpha-synuclein in the assembly of fibrils." Biochemistry 46(46): 13322-13330.

Ralston, G. B. (1990). "Effects of Crowding in Protein Solutions." Journal of Chemical Education 67(10): 857-860.

Raman, B., E. Chatani, et al. (2005). "Critical balance of electrostatic and hydrophobic interactions is required for beta 2-microglobulin amyloid fibril growth and stability." Biochemistry 44(4): 1288-1299.

Rao, J. N., C. C. Jao, et al. (2010). "A combinatorial NMR and EPR approach for evaluating the structural ensemble of partially folded proteins." Journal of the American Chemical Society 132(25): 8657-8668.

Reddy, P. H., S. McWeeney, et al. (2004). "Gene expression profiles of transcripts in amyloid precursor protein transgenic mice: up-regulation of mitochondrial metabolism and apoptotic genes is an early cellular change in Alzheimer's disease." Human Molecular Genetics 13(12): 1225-1240.

Regensburger, M., I. Prots, et al. (2014). "Adult hippocampal neurogenesis in Parkinson's disease: impact on neuronal survival and plasticity." Neural Plasticity 2014: 454696.

Rhinn, H., L. Qiang, et al. (2012). "Alternative alpha-synuclein transcript usage as a convergent mechanism in Parkinson's disease pathology." Nature Communications 3: 1084.

Riener, C. K., G. Kada, et al. (2002). "Quick measurement of protein sulfhydryls with Ellman's reagent and with 4,4'-dithiodipyridine." Analytical and Bioanalytical Chemistry 373(4-5): 266-276.

Rodriguez, J. A., M. I. Ivanova, et al. (2015). "Structure of the toxic core of alphasynuclein from invisible crystals." Nature 525(7570): 486-490.

Roostaee, A., S. Beaudoin, et al. (2013). "Aggregation and neurotoxicity of recombinant alpha-synuclein aggregates initiated by dimerization." Molecular Neurodegeneration 8: 5. 
Rosi, N. L. and C. A. Mirkin (2005). "Nanostructures in biodiagnostics." Chemical Reviews 105(4): 1547-1562.

Sahay, S., D. Ghosh, et al. (2015). "Familial Parkinson Disease-associated Mutations Alter the Site-specific Microenvironment and Dynamics of alpha-Synuclein." Journal of Biological Chemistry 290(12): 7804-7822.

Sandberg, A., L. M. Luheshi, et al. (2010). "Stabilization of neurotoxic Alzheimer amyloid-beta oligomers by protein engineering." Proceedings of the National Academy of Sciences of the United States of America 107(35): 15595-15600.

Sarangi, S. N., K. Goswami, et al. (2007). "Biomolecular recognition in DNA tagged CdSe nanowires." Biosensors and Bioelectronics 22(12): 3086-3091.

Scheibel, T., A. S. Kowal, et al. (2001). "Bidirectional amyloid fiber growth for a yeast prion determinant." Current Biology 11(5): 366-369.

Scheibel, T., R. Parthasarathy, et al. (2003). "Conducting nanowires built by controlled self-assembly of amyloid fibers and selective metal deposition." Proceedings of the National Academy of Sciences of the United States of America 100(8): 45274532.

Semerdzhiev, S. A., D. R. Dekker, et al. (2014). "Self-assembly of protein fibrils into suprafibrillar aggregates: bridging the nano- and mesoscale." ACS nano 8(6): 5543-5551.

Serpell, L. C., J. Berriman, et al. (2000). "Fiber diffraction of synthetic alpha-synuclein filaments shows amyloid-like cross-beta conformation." Proceedings of the National Academy of Sciences of the United States of America 97(9): 48974902.

Sherman, M. Y. and A. L. Goldberg (2001). "Cellular defenses against unfolded proteins: a cell biologist thinks about neurodegenerative diseases." Neuron 29(1): 15-32.

Shikiya, R. A., J. I. Ayers, et al. (2010). "Coinfecting prion strains compete for a limiting cellular resource." Journal of Virology 84(11): 5706-5714.

Shtilerman, M. D., T. T. Ding, et al. (2002). "Molecular crowding accelerates fibrillization of alpha-synuclein: could an increase in the cytoplasmic protein concentration induce Parkinson's disease?" Biochemistry 41(12): 3855-3860.

Shvadchak, V. V., M. M. Claessens, et al. (2015). "Fibril breaking accelerates alphasynuclein fibrillization." Journal of Physical Chemistry B 119(5): 1912-1918.

Sidhu, A., I. Segers-Nolten, et al. (2014). "Solution conditions define morphological homogeneity of alpha-synuclein fibrils." Biochimica et Biophysica Acta 1844(12): 2127-2134.

Singleton, A. B., M. Farrer, et al. (2003). "alpha-Synuclein locus triplication causes Parkinson's disease." Science 302(5646): 841. 
Slotta, U., S. Hess, et al. (2007). "Spider silk and amyloid fibrils: a structural comparison." Macromolecular Bioscience 7(2): 183-188.

Smith, J. F., T. P. Knowles, et al. (2006). "Characterization of the nanoscale properties of individual amyloid fibrils." Proceedings of the National Academy of Sciences of the United States of America 103(43): 15806-15811.

Snead, D. and D. Eliezer (2014). "Alpha-synuclein function and dysfunction on cellular membranes." Experimental Neurobiology 23(4): 292-313.

Sperling, R. A. and W. J. Parak (2010). "Surface modification, functionalization and bioconjugation of colloidal inorganic nanoparticles." Philosophical transactions. Series A, Mathematical, physical, and engineering sciences 368(1915): 1333-1383.

Spillantini, M. G., M. L. Schmidt, et al. (1997). "Alpha-synuclein in Lewy bodies." Nature 388(6645): 839-840.

Spillantini, M. G., R. A. Crowther, et al. (1998). "alpha-Synuclein in filamentous inclusions of Lewy bodies from Parkinson's disease and dementia with lewy bodies." Proceedings of the National Academy of Sciences of the United States of America 95(11): 6469-6473.

Stefanis, L. (2012). "alpha-Synuclein in Parkinson's disease." Cold Spring Harbour Perspective in Medicine 2(2): a009399.

Stevens, R., L. Stevens, et al. (1983). "The Stabilities of Various Thiol Compounds Used in Protein Purifications." Biochemical Education 11(2): 70-70

Straub, J. E. and D. Thirumalai (2011). "Toward a molecular theory of early and late events in monomer to amyloid fibril formation." Annual Review of Physical Chemistry 62: 437-463.

Sung, Y. H., C. Rospigliosi, et al. (2006). "NMR mapping of copper binding sites in alphasynuclein." Biochimica et Biophysica Acta 1764(1): 5-12.

Sweers, K. K., K. O. van der Werf, et al. (2012). "Atomic force microscopy under controlled conditions reveals structure of C-terminal region of alpha-synuclein in amyloid fibrils." ACS nano 6(7): 5952-5960.

Takeda, A., M. Mallory, et al. (1998). "Abnormal accumulation of NACP/alphasynuclein in neurodegenerative disorders." American Journal of Pathology 152(2): 367-372.

Tanaka, H., A. Herland, et al. (2008). "Enhanced current efficiency from bio-organic light-emitting diodes using decorated amyloid fibrils with conjugated polymer." Nano Letters 8(9): 2858-2861. 
Tanaka, M., P. Chien, et al. (2005). "Mechanism of cross-species prion transmission: an infectious conformation compatible with two highly divergent yeast prion proteins." Cell 121(1): 49-62.

Tanaka, M., S. R. Collins, et al. (2006). "The physical basis of how prion conformations determine strain phenotypes." Nature 442(7102): 585-589.

Teich, A. F., M. Patel, et al. (2013). "A reliable way to detect endogenous murine betaamyloid." PLoS ONE 8(2): e55647.

Trexler, A. J. and E. Rhoades (2009). "Alpha-synuclein binds large unilamellar vesicles as an extended helix." Biochemistry 48(11): 2304-2306.

Trexler, A. J. and E. Rhoades (2012). "N-Terminal acetylation is critical for forming alpha-helical oligomer of alpha-synuclein." Protein Science 21(5): 601-605.

Trifunovic, A. (2006). "Mitochondrial DNA and ageing." Biochimica et Biophysica Acta 1757(5-6): 611-617.

Truong, L., S. C. Tilton, et al. (2013). "Surface functionalities of gold nanoparticles impact embryonic gene expression responses." Nanotoxicology 7(2): 192-201.

Tsoli, M., H. Kuhn, et al. (2005). "Cellular uptake and toxicity of Au55 clusters." Small 1(8-9): 841-844.

Tycko, R. (2014). "Physical and structural basis for polymorphism in amyloid fibrils." Protein Science 23(11): 1528-1539.

Ueda, K., T. Saitoh, et al. (1994). "Tissue-dependent alternative splicing of mRNA for NACP, the precursor of non-A beta component of Alzheimer's disease amyloid." Biochemical and Biophysical Research Communications 205(2): 1366-1372.

Ulmer, T. S., A. Bax, et al. (2005). "Structure and dynamics of micelle-bound human alpha-synuclein." Journal of Biological Chemistry 280(10): 9595-9603.

Usov, I., J. Adamcik, et al. (2013). "Polymorphism complexity and handedness inversion in serum albumin amyloid fibrils." ACS nano 7(12): 10465-10474.

Uversky, V. N. (2010). "Mysterious oligomerization of the amyloidogenic proteins." FEBS Journal 277(14): 2940-2953.

Uversky, V. N. (2011). "Intrinsically disordered proteins from A to Z." International Journal of Biochemistry and Cell Biology 43(8): 1090-1103.

Uversky, V. N., J. Li, et al. (2001). "Evidence for a partially folded intermediate in alphasynuclein fibril formation." Journal of Biological Chemistry 276(14): 1073710744.

Uversky, V. N., J. Li, et al. (2001). "Metal-triggered structural transformations, aggregation, and fibrillation of human alpha-synuclein. A possible molecular 
NK between Parkinson's disease and heavy metal exposure." Journal of Biological Chemistry 276(47): 44284-44296.

van Raaij, M. E., I. M. Segers-Nolten, et al. (2006). "Quantitative morphological analysis reveals ultrastructural diversity of amyloid fibrils from alpha-synuclein mutants." Biophysical Journal 91(11): L96-98.

van Raaij, M. E., J. van Gestel, et al. (2008). "Concentration dependence of alphasynuclein fibril length assessed by quantitative atomic force microscopy and statistical-mechanical theory." Biophysical Journal 95(10): 4871-4878.

van Rooijen, B. D., M. M. Claessens, et al. (2009). "Lipid bilayer disruption by oligomeric alpha-synuclein depends on bilayer charge and accessibility of the hydrophobic core." Biochimica et Biophysica Acta 1788(6): 1271-1278.

Vanik, D. L., K. A. Surewicz, et al. (2004). "Molecular basis of barriers for interspecies transmissibility of mammalian prions." Molecular Cell 14(1): 139-145.

Vilar, M., H. T. Chou, et al. (2008). "The fold of alpha-synuclein fibrils." Proceedings of the National Academy of Sciences of the United States of America 105(25): 8637-8642.

Viles, J. H. (2012). "Metal ions and amyloid fiber formation in neurodegenerative diseases. Copper, zinc and iron in Alzheimer's, Parkinson's and prion diseases." Coordination Chemistry Reviews 256(19-20): 2271-2284.

Volles, M. J. and P. T. Lansbury, Jr. (2002). "Vesicle permeabilization by protofibrillar alpha-synuclein is sensitive to Parkinson's disease-linked mutations and occurs by a pore-like mechanism." Biochemistry 41(14): 4595-4602.

Volles, M. J., S. J. Lee, et al. (2001). "Vesicle permeabilization by protofibrillar alphasynuclein: implications for the pathogenesis and treatment of Parkinson's disease." Biochemistry 40(26): 7812-7819.

Wang, M. S., S. Boddapati, et al. (2010). "Curcumin reduces alpha-synuclein induced cytotoxicity in Parkinson's disease cell model." BMC Neuroscience 11: 57.

Watt, B., G. van Niel, et al. (2013). "PMEL: a pigment cell-specific model for functional amyloid formation." Pigment Cell and Melanoma Research 26(3): 300-315.

Watts, J. C., K. Giles, et al. (2013). "Transmission of multiple system atrophy prions to transgenic mice." Proceedings of the National Academy of Sciences of the United States of America 110(48): 19555-19560.

Webb, J. L., B. Ravikumar, et al. (2003). "Alpha-Synuclein is degraded by both autophagy and the proteasome." Journal of Biological Chemistry 278(27): 25009-25013.

Wolfe, L. S., M. F. Calabrese, et al. (2010). "Protein-induced photophysical changes to the amyloid indicator dye thioflavin T." Proceedings of the National Academy of Sciences of the United States of America 107(39): 16863-16868. 
Wood, S. J., J. Wypych, et al. (1999). "alpha-synuclein fibrillogenesis is nucleationdependent. Implications for the pathogenesis of Parkinson's disease." Journal of Biological Chemistry 274(28): 19509-19512.

Wordehoff, M. M., O. Bannach, et al. (2015). "Single fibril growth kinetics of alphasynuclein." Journal of Molecular Biology 427(6 Pt B): 1428-1435.

Wright, P. E. and H. J. Dyson (1999). "Intrinsically unstructured proteins: re-assessing the protein structure-function paradigm." Journal of Molecular Biology 293(2): 321-331.

Wu, C., M. Biancalana, et al. (2009). "Binding modes of thioflavin-T to the single-layer beta-sheet of the peptide self-assembly mimics." Journal of Molecular Biology 394(4): 627-633.

Wu, C., Z. Wang, et al. (2007). "Dual binding modes of Congo red to amyloid protofibril surface observed in molecular dynamics simulations." Journal of the American Chemical Society 129(5): 1225-1232.

Xie, Y. Y., C. J. Zhou, et al. (2010). "Interaction with synphilin-1 promotes inclusion formation of alpha-synuclein: mechanistic insights and pathological implication." FASEB Journal 24(1): 196-205.

Xue, W. F., A. L. Hellewell, et al. (2009). "Fibril fragmentation enhances amyloid cytotoxicity." Journal of Biological Chemistry 284(49): 34272-34282.

Xue, W. F., S. W. Homans, et al. (2009). "Amyloid fibril length distribution quantified by atomic force microscopy single-particle image analysis." Protein Engineering, Design and Selection 22(8): 489-496.

Yamin, G., L. A. Munishkina, et al. (2005). "Forcing nonamyloidogenic beta-synuclein to fibrillate." Biochemistry 44(25): 9096-9107.

Yan, H., S. H. Park, et al. (2003). "DNA-templated self-assembly of protein arrays and highly conductive nanowires." Science 301(5641): 1882-1884.

Yonetani, M., T. Nonaka, et al. (2009). "Conversion of wild-type alpha-synuclein into mutant-type fibrils and its propagation in the presence of A30P mutant." Journal of Biological Chemistry 284(12): 7940-7950.

Yoon, G., M. Lee, et al. (2014). "Role of sequence and structural polymorphism on the mechanical properties of amyloid fibrils." PLoS ONE 9(2): e88502.

Zareba, M., A. Bober, et al. (1995). "The effect of a synthetic neuromelanin on yield of free hydroxyl radicals generated in model systems." Biochimica et Biophysica Acta 1271(2-3): 343-348.

Zarranz, J. J., J. Alegre, et al. (2004). "The new mutation, E46K, of alpha-synuclein causes Parkinson and Lewy body dementia." Annals of Neurology 55(2): 164-173. 
Zecca, L., D. Tampellini, et al. (2001). "Substantia nigra neuromelanin: structure, synthesis, and molecular behaviour." Molecular Pathology 54(6): 414-418.

Zhao, M., D. Cascio, et al. (2011). "Structures of segments of alpha-synuclein fused to maltose-binding protein suggest intermediate states during amyloid formation." Protein Science 20(6): 996-1004.

Zhou, J., A. Saha, et al. (2015). "Macroscopic single-crystal gold microflakes and their devices." Advanced Materials 27(11): 1945-1950.

Zijlstra, N., M. M. Claessens, et al. (2014). "Elucidating the aggregation number of dopamine-induced alpha-synuclein oligomeric assemblies." Biophysical Journal 106(2): 440-446.

Zou, Y., Y. Li, et al. (2013). "Parallel beta-sheet fibril and antiparallel beta-sheet oligomer: new insights into amyloid formation of hen egg white lysozyme under heat and acidic condition from FTIR spectroscopy." Journal of Physical Chemistry B 117(15): 4003-4013. 


\section{Summary}

In the research described in this thesis we have investigated some of the important yet puzzling aspects of amyloids formed by aSyn protein. The main line of questions and the ensuing experiments have attempted to understand the significance and the implications of the different morphologies observed in an aggregation reaction. aSyn monomers, like other amyloid proteins, form polymorphic fibrils in a de novo aggregation. To be able to comprehend the biophysical and biochemical characteristics of these morphologies it is important to be able to form fibrils of one morphology and then study them.

In chapter 1, we presented the key features of amyloids in terms of their growth, biophysical and biochemical properties. Then we introduced the model protein of the thesis, aSyn. We summarized the relevant literature and discussed the accepted physiological relevance, structure and polymorphism of aSyn fibrils. In chapter 2, we showed that by varying the solution conditions, $\alpha$ Syn monomers can be induced to form a homogeneous population of fibrils. The results suggest that low salt conditions reduce heterogeneity. The low salt concentration is believed to influence the charge screening in the soluble protein molecules, which affects the repertoire of conformations accessible to the unfolded monomer. Moreover, inclusion of EDTA in the aggregation solution appreciably improves homogeneity of the fibrils produced. The presence of EDTA possibly sequesters the trace divalent metal ions that may interact with the negatively charged C-terminus of the soluble monomer and thus modify the aggregation prone conformations.

In chapter 3, we prepared fibrils from wt and the disease mutants (A30P, E46K and A53T) aSyn using the aggregation protocol optimized in chapter 2 and studied their morphology over a period of one year. The longitudinal morphological study provided interesting insights into the mechanisms that govern the morphology of the fibrils. During the early phase of aggregation, all the sequences show polymorphism however with progress of fibrillization, in our aggregation conditions, one of the polymorphs out competed the others and formed a homogeneous population of fibrils at the plateau phase. The fibrils from wt, A30P, E46K and A53T showed characteristic morphology at the plateau phase. Additionally, the wt fibrils slowly changed from fibrils with $\approx 100 \mathrm{~nm}$ 
periodicity to fibrils with longer periodicity of $\approx 320 \mathrm{~nm}$ in 3 months. The morphology of the wt fibrils continued to mature up to 6 months resulting in mainly non-periodic and heterogeneous fibrils. The disease mutants however, did not change over time.

In chapter 4, we studied morphology specific characteristics of the fibrils with regard to ThT fluorescence intensity. Fibrils of two distinct morphologies but same protein monomers (wt) were produced by templating on seeds of A30P and A53T fibrils. The relative ThT fluorescence intensities in the fibrils with different morphology did not correlate with the respective amount of monomer incorporation. Analysis of the ThT fluorescence lifetimes when bound to morphologically distinct fibrils showed different binding surfaces for the two morphologies that lead to typical ThT fluorescence intensities.

In chapter 5, we investigated the effect of the seed and monomer conformations on heterologous seeded aggregations. Seeds of wt, A30P and A53T fibrils were aggregated in homologous and heterologous aggregation with wt, A30P and A53T monomers. The wt monomers aggregated with all the seed sequences while the aggregation of $\mathrm{A} 30 \mathrm{P}$ and A53T monomers was restricted to wt or homologous seeds. Thus the conformations available to the soluble monomers are a critical determinant for heterologous aggregations. In chapter 6, we explored the functionalization of A140C aSyn fibrils with gold nanoparticles of 1.4 and $5 \mathrm{~nm}$. We showed that the fibrils can be successfully functionalized with these gold nanoparticles and that the particle size can be increased by electroless gold enhancement. The hybrid bio-nanostructures show potential for conductive properties when probed with contact probe AFM. In chapter 7, we discuss the work presented in this thesis and speculated on the possible future directions. 


\section{Samenvatting}

In het onderzoek, beschreven in dit proefschrift, hebben we enkele belangrijke, nog onopgeloste aspecten, bestudeerd van amyloïde structuren gevormd door het eiwit alpha-synucleine ( $\alpha$ Syn). De vragen en daaruit voortvloeiende experimenten zijn er vooral op gericht geweest om meer inzicht te verkrijgen in de betekenis en de gevolgen van de verschillende morfologische vormen van fibrillen, die in een aggregatie reactie waargenomen worden. aSyn monomeren vormen, evenals andere amylö̈de eiwitten, polymorfe fibrillen in een de novo aggregatie. Om de biofysische en biochemische eigenschappen van deze morfologische vormen te kunnen begrijpen is het belangrijk om fibrillen te kunnen produceren met één uniforme morfologie en deze dan te bestuderen.

In hoofdstuk 1 introduceren we de belangrijkste kenmerken van amyloids met betrekking tot hun groei en biofysische en biochemische eigenschappen. Vervolgens introduceren we aSyn, het model eiwit gebruikt in dit proefschrift. We vatten de relevante literatuur samen en bediscussiëren de tot dusver erkende fysiologische relevantie, evenals de structuur en polymorfisme van aSyn fibrillen. In hoofdstuk 2 laten we zien dat $\alpha$ Syn monomeren aangezet kunnen worden tot het vormen van een morfologisch homogene populatie fibrillen door specifieke reactie omstandigheden te kiezen. De resultaten geven aan dat lage zout condities de heterogeniteit verminderen. Er wordt verondersteld dat lage zout concentraties de ladingsafscherming binnen het oplosbare eiwit molecuul beïnvloeden, wat effect heeft op het scala aan toegankelijke conformaties voor het ongevouwen monomeer. Wanneer bovendien EDTA aan de aggregatie oplossing toegevoegd wordt, blijkt de homogeniteit van de geproduceerde fibrillen aanzienlijk te verbeteren. Het aanwezige EDTA wordt verondersteld divalente metaal ionen, die in sporen hoeveelheden aanwezig zijn en interactie hebben met de negatief geladen $\mathrm{C}$-terminus van het oplosbare $\alpha$ Syn monomeer, weg te vangen. Dit kan een andere samenstelling van de populatie van conformaties, die tot aggregatie kunnen leiden, tot gevolg hebben.

In hoofdstuk 3 hebben we fibrillen bereid van wildtype en ziekte gerelateerde mutanten (A30P, E46K and A53T) van aSyn volgens het aggregatie protocol, dat we in hoofdstuk 2 geoptimaliseerd hebben. We hebben de morfologie van deze fibrillen over 
een periode van één jaar gevolgd. Deze studie over langere tijd, heeft interessante inzichten verschaft in de mechanismes, die de morfologie van aggregerende fibrillen sturen. In de beginfase van aggregatie vertonen alle aSyn sequenties polymorfisme. Echter, met het voortgaan van de fibrilvorming onder de gekozen aggregatie condities, blijkt zich in de plateau fase een morfologisch homogene populatie fibrillen te vormen, ten koste van de overige polymorfe structuren. De wildtype, A30P, E46K en A53T fibrillen vertonen karakteristieke morfologie in de plateau fase. Verder blijken wildtype fibrillen langzaam te veranderen over een periode van 3 maanden van fibrillen met $\approx 100$ $\mathrm{nm}$ periodiciteit naar fibrillen met een langere periodiciteit van $\approx 320 \mathrm{~nm}$. De morfologie van de wildtype fibrillen blijft zich door ontwikkelen tot aan 6 maanden, hoofdzakelijk resulterend in fibrillen zonder periodiciteit en heterogene fibrillen. Echter, de fibrillen gevormd uit de ziekte gerelateerde eiwit mutanten veranderen niet van morfologie in de tijd.

In hoofdstuk 4 hebben we morfologie specifieke kenmerken van fibrillen bestudeerd met betrekking tot ThT binding op basis van gemeten ThT fluorescentie parameters. Twee morfologisch verschillende fibril populaties, opgebouwd uit dezelfde eiwit monomeren (wildtype), werden geproduceerd door A30P and A53T fibrillen te gebruiken als seeds. De relatieve ThT fluorescentie intensiteiten gemeten voor fibrillen met verschillende morfologie blijken niet te correleren met de hoeveelheid ingebouwd monomeer. Analyse van de fluorescentie levensduur van fibril-gebonden ThT geeft aan dat morfologisch verschillende fibrillen verschillende bindingsplaatsen hebben aan hun oppervlak die leiden tot morfologie specifieke ThT fluorescentie intensiteiten.

In hoofdstuk 5 hebben we het effect van seed en monomeer conformaties onderzocht in heterologe aggregaties. Seeds van wildtype, A30P en A53T fibrillen werden geaggregeerd in homologe en heterologe aggregaties met wildtype, A30P en A53T monomeren. De wildtype monomeren aggregeerden met alle typen seeds tot fibrillen, terwijl de aggregatie van A30P en A53T monomeren beperkt was tot wildtype of homologe seeds. Hieruit blijkt dat de conformaties, die het oplosbare monomeer aan kan nemen, bepalend zijn voor heterologe aggregaties. In hoofdstuk 6 hebben we de functionalisatie van A140C aSyn fibrillen met 1.4 en $5 \mathrm{~nm}$ goud nanodeeltjes bestudeerd. We hebben laten zien dat de fibrillen succesvol gefunctionaliseerd kunnen worden en dat de afmeting van de gebonden goud deeltjes vergroot kan worden door middel van een chemisch proces. De hybride bio-nanostructuren vertoonden elektrisch 
geleidende eigenschappen bij gebruik van contact-probe AFM. In hoofdstuk 7 bediscussiëren we het werk beschreven in dit proefschrift en speculeren we over mogelijke richtingen voor toekomstig onderzoek. 


\section{Acknowledgements}

It has been an immense pleasure working on this thesis with wonderful bosses and colleagues.

Foremost I would like to thank you Vinod and Ine for this opportunity. Your constant encouragement and appreciation has been instrumental in shaping my outlook for the amyloid science. When I joined, I was a novice to the system and the techniques. I can hardly express my gratitude to Ine for teaching me the staple techniques of the lab and for being so patient with me, especially for introduction on AFM II (I remember taking the introduction at least 3 times and I still didn't use the instrument). Vinod you are a trove of knowledge and have meticulous tips for writing. I wish I could learn more from you. Alas, you left us for the glitz of Amsterdam. As they say when one boss leaves you another one adopts you. And I found Mireille as my new boss in the group. Mireille your inputs on the manuscripts were very timely and valuable to me. In all, Vinod, Ine and Mireille the numerous discussions on the results and the potential experiments is really the heart of this thesis. Thank you.

The next pit stop for any person joining the NBP group is the warm Ms Winters. Sylvia you are literally the charm of the group and when Syl is ill the feminine voice of NBP is sorely missed. Thank you for all the information and the warmth.

Many experiments described in this thesis took me to sage and experienced people beyond the triumvirate of my bosses and the group.

Prof. Wilfred van der Wiel, the different conductivity experiments over the past two years on the possible nanowires with your group members (Martin, Serkan, Robin and Celestine) was a pleasant and insightful experience. Thank you. I would like to extend a special thanks to Martin for all the help and advice.

Dr. Vincent Raussens, learning FTIR at your lab in Brussels was a privilege that I am sure will be useful for me in the future. Thanks a lot.

At closer quarters, learned advice and discussions with Christian, Hans, Kerensa and Ron, have contributed to better experiments and broadened my understanding of the corresponding subjects. Thank you.

Large part of my thesis work involved AFM and as a novice I often looked up to Kees, Martin and Robert for help in imaging and interpretation of the images. Prompt 
and erudite guidance from "The AFM guys" ensured smooth running of my experiments. In addition to AFM, Robert you were a great support for sputtering my samples for the nanowire experiments, often at very short notices. Thank you.

The girl power of the group is the fuel of my PhD. Kirsten, Nathalie and Yvonne you are fabulous with all matters proteinaceous. I am indebted to you for your support in the lab. Irene, I didn't work much with you but on the few occasions that I seeked your help, you always exceeded my expectations. Thank you. In all, girls you rock!

My office mates Chris, Frederica, Harmen and Maurice, I had a very nice time in our office, apt for work and chats on myriads of subjects - long and short, serious and silly. Harmen a special thanks to you for the knick-knacks we shared while writing our theses, through printing to our pending defences. If my office was convivial, the other group mates were certainly not behind. Aditya, Amin, Anja, Burcu, Carla, Himanshu, Jord, Josey, Kristian, Lantian, Lisanne, Ramesh, Remco, Saskia, Senthil and Slav, a big thank you for all the help and bon-homie. I singularly appreciate the warm technical assistance I received during my initial days at NBP by Kim, Yves and Martin Stöckl.

Few people in a boat, to say everything of life: DP, SB, SC, SN, VP and YS, you make life more interesting, theoretically as well as practically. No matter the distance, we can always find ways to comfort and confuse each other. I acknowledge you here but no thanks yet.

My folks, DSS, HS, NSS, PS and SS (×3). Keep up the good work ;).

The much obliged, Arshdeep 


\section{The blind men and the elephant}

It was six men of Indostan, to learning much inclined, who went to see the elephant (Though all of them were blind),

that each by observation, might satisfy his mind.

The first approached the elephant, and, happening to fall, against his broad and sturdy side, at once began to bawl:

'God bless me! but the elephant, is nothing but a wall!'

The second feeling of the tusk, cried: 'Ho! what have we here, so very round and smooth and sharp? To me tis mighty clear,

this wonder of an elephant, is very like a spear!'

The third approached the animal, and, happening to take,

the squirming trunk within his hands, 'I see,' quoth he, the elephant is very like a snake!'

The fourth reached out his eager hand, and felt about the knee: 'What most this wondrous beast is like, is mighty plain,' quoth he;

'Tis clear enough the elephant is very like a tree.'

The fifth, who chanced to touch the ear, Said; 'E'en the blindest man can tell what this resembles most; Deny the fact who can,

This marvel of an elephant, is very like a fan!'

The sixth no sooner had begun, about the beast to grope, than, seizing on the swinging tail, that fell within his scope,

'I see,' quothe he, 'the elephant is very like a rope!'

And so these men of Indostan, disputed loud and long,

each in his own opinion, exceeding stiff and strong,

Though each was partly in the right, and all were in the wrong!

So, oft in theologic wars, the disputants, I ween, tread on in utter ignorance, of what each other mean, and prate about the elephant, not one of them has seen!

-John Godfrey Saxe, based on an Indian folktale.

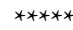






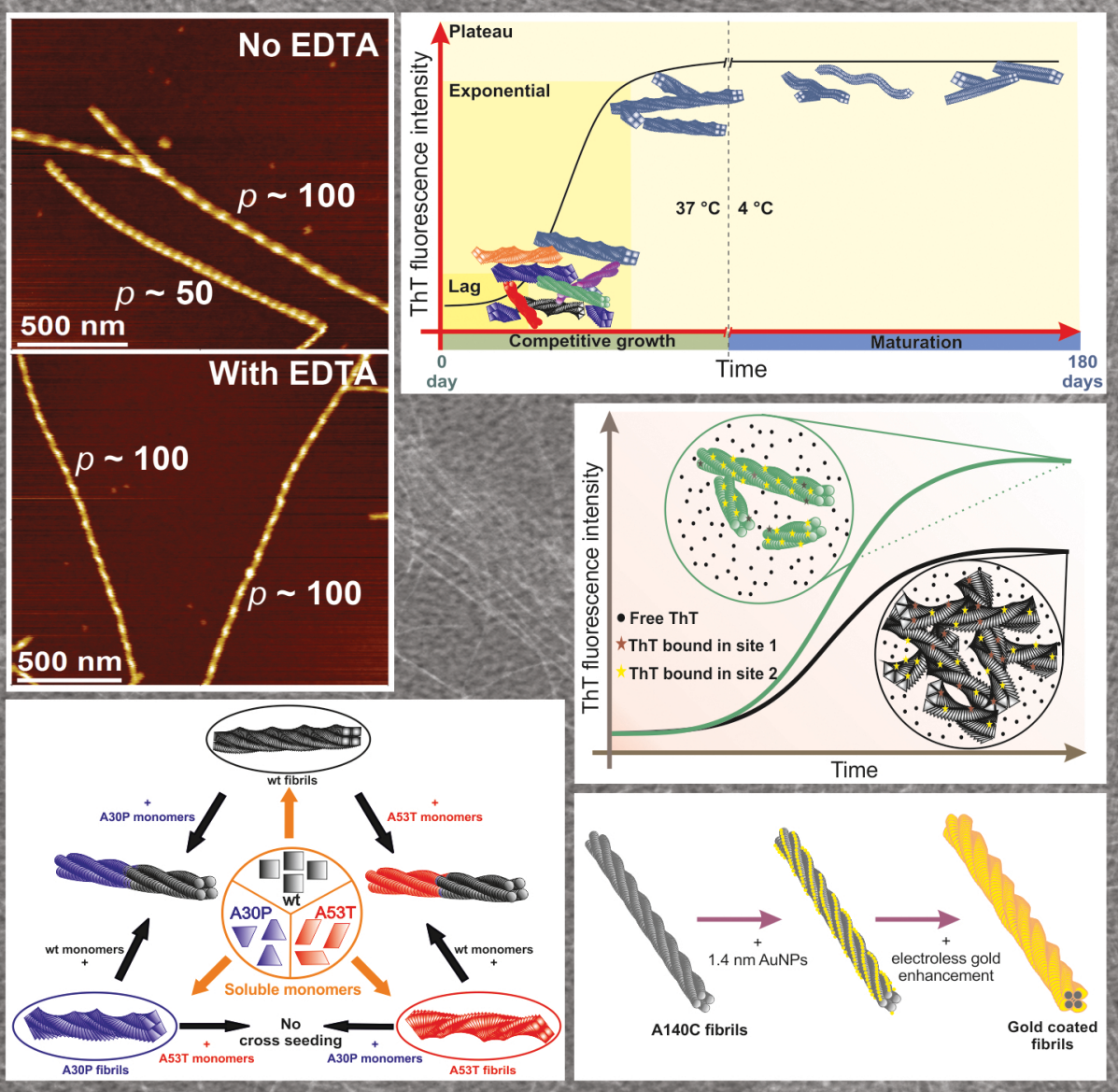

ISBN $978-90-365-4024-7$

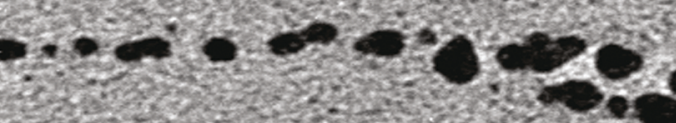

